\title{
High-Resolution Spectroscopic Diagnostics of Very High-Temperature Plasmas in the Hard X-ray Regime
}

\author{
K. Widmann \\ Ph.D. Thesis
}

\section{December 6, 1999}

U.S. Department of Energy

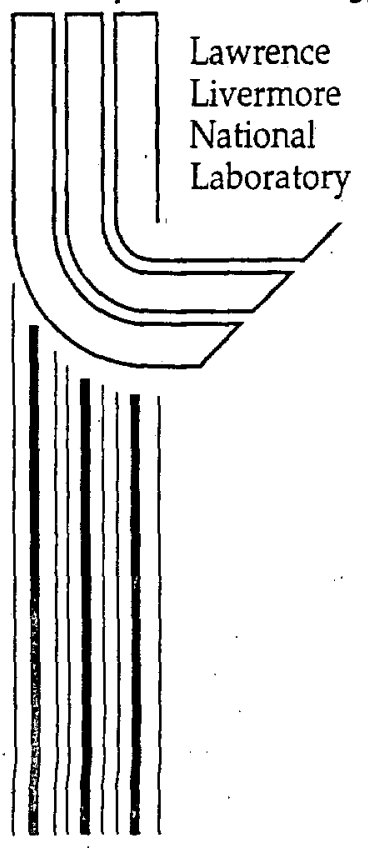




\section{DISCLAIMER}

This document was prepared as an account of work sponsored by an agency of the United States Government. Neither the United States Government nor the University of California nor any of their employees, makes any warranty, express or implied, or assumes any legal liability or responsibility for the accuracy, completeness, or usefulness of any information, apparatus, product, or process disclosed, or represents that its use would not infringe privately owned rights. Reference herein to any specific commercial product, process, or service by trade name, trademark, manufacturer, or otherwise, does not necessarily constitute or imply its endorsement, recommendation, or favoring by the United States Government or the University of California. The views and opinions of authors expressed herein do not necessarily state or reflect those of the United States Government or the University of California, and shall not be used for advertising or product endorsement purposes.

Work performed under the auspices of the U. S. Department of Energy by the University of California Lawrence Livermore National Laboratory under Contract W-7405-Eng-48.

This report has been reproduced

directly from the best available copy.

Available to DOE and DOE contractors from the

Office of Scientific and Technical Information

P.O. Box 62, Oak Ridge, TN 37831

Prices available from (423) 576-8401

http://apollo.osti.gov/bridge/

Available to the public from the

National Technical Information Service

U.S. Department of Commerce

5285 Port Royal Rd.,

Springfield, VA 22161

http://www.ntis.gov/

\section{OR}

Lawrence Livermore National Laboratory

Technical Information Department's Digital Library

http://www.llnl.gov/tid/Library.html 


\section{HIGH-RESOLUTION SPECTROSCOPIC DIAGNOSTICS OF VERY HIGH-TEMPERATURE PLASMAS IN THE HARD X-RAY REGIME}

Dissertation

Klaus Widmann

Technische Universität Graz

1998 


$$
\begin{gathered}
1^{\text {st }} \text { edition - typesetted for DIN A4 size } \\
2^{\text {nd }} \text { edition - typesetted for US Letter size }
\end{gathered}
$$

(c) 1998

Klaus Widmann

ALL RIGHTS RESERVED 


\section{Überblick}

Die harte Röntgenemission hochgeladener Krypton und Xenon Ionen wurde mittels konventioneller und neuentwickelter Kristallspektrometer der von Hámos und DuMond Geometrie feinstrukturmäßig aufgelöst und ihre Anwendung in Hinblick auf die Elektronen- und Ionentemperaturdiagnostik sowie die Ladungsverteilungsgleichgewichtbestimmung von Hochtemperaturplasmen untersucht. Die Experimente, die an den nieder- und hochenergetischen Elektronenstrahlionenfallen des Lawrence Livermore National Laboratory durchgeführt wurden, umfassen die Vermessung der etwa $0.1 \mathrm{~nm}$ langen Wellenlängen der charakteristischen $\mathrm{K}$-Schalenemission des heliumartigen Kryptons $\left(\mathrm{Kr}^{34+}\right)$ und des durch dielektronische Rekombination innerschalenangeregten lithiumartigen Kryptons $\left(\mathrm{Kr}^{33+}\right)$, sowie die Vermessung der Übergangsenergien und Elektronenstoßanregungswirkungsquerschnitte der Resonanzlinien des helium- und wasserstoffartigen Xenons $\left(\mathrm{Xe}^{52+}, \mathrm{Xe}^{53+}\right)$ im $30 \mathrm{keV}$ Bereich. Die anregungsenergieselektive Untersuchung der dielektronischen KLL Resonanzen des hochgeladenen Krypton Ions ermöglicht die genaue Interpretation der bei spektroskopischer Untersuchung von Plasmen erhaltenen Linienprofile dieser charakteristischen Emissionslinien. . Die Gegenüberstellung der gemessenen Elektronenstoßanregungswirkungsquerschnitte des heliumartigen Xenons mit den theoretischen Werten zeigt deutlich die Notwendigkeit der Miteinbeziehung der Breit Wechselwirkung.

Fachgebiet(e): Atom, Flüssigkeiten und Plasma, Optik;

Schlagwörter: Röntgenspektroskopie, Kristallspektrometer, Transmissionskristallspektrometer, hochgeladene Ionen, Elektronenstrahlionenfalle, relativistische und quantenelektrodynamische Effekte in Atomen, Elektron-Ion Wechselwirkung, dielektronische Rekombination, Strahlungsrekombination, Ladungsaustausch, Krypton, Xenon; 


\begin{abstract}
Motivated by the need for establishing a reliable database useful for the application of $\mathrm{x}$-ray spectroscopic tools for the diagnostic of very high temperature plasmas, highresolution crystal spectrometer measurements have been performed investigating the characteristic $\mathrm{K}$-shell radiation of highly charged krypton and xenon. The measurements, which have been performed at the Electron-Beam-Ion-Trap (EBIT) facility of the Lawrence Livermore National Laboratory ${ }^{1}$, include the investigation of the $n$ $=2 \rightarrow 1$ transitions in heliumlike krypton $\left(\mathrm{Kr}^{34+}\right)$ and innershell excited lithiumlike krypton $\left(\mathrm{Kr}^{33+}\right)$ utilizing a conventional reflection-type crystal spectrometer of von Hámos geometry. The electron-excitation-energy selective measurements map the contribution of the dielectronic recombination lines providing the means of accurate interpretation of the line profiles of the characteristic $\mathrm{K} \alpha \mathrm{x}$-ray emission of plasmas. The high-resolution measurements of the $\mathrm{n}=2 \rightarrow 1$ transitions in heliumlike xenon $\left(\mathrm{Xe}^{52+}\right)$ and hydrogenlike xenon $\left(\mathrm{Xe}^{53+}\right)$ were based on a new transmission-type crystal spectrometer of DuMond geometry. The resolving power of the developed spectrometer was sufficient for charge state specific observation allowing the determination of the electron-impact excitation cross section for the hydrogen- and heliumlike $\mathrm{K} \alpha$ transitions. The disagreement with theoretically predicted values is a measure of the magnitude of the Breit interaction for the highly charged high $-Z$ ions.
\end{abstract}

Subject(s): Atomic, Fluid and Plasma, Optics;

Keywords: X-ray spectroscopy, crystal spectrometer, transmission-type crystal spectrometer, highly charged ions, electron beam ion trap, relativistic and quantum electrodynamic effects in atoms, electron-ion interaction, dielectronic recombination, radiative recombination, charge exchange, krypton, xenon;

\footnotetext{
${ }^{1}$ This work was performed under the auspices of the United States Department of Energy by Lawrence Livermore National Laboratory under contract number W-7405-ENG-48 and supported during the first year by the Office of Fusion Energy of the Department of Energy, Division of Applied Plasma Physics.
} 


\section{Contents}

1 Introduction $\quad 1$

2 Plasma diagnostics based on x-ray spectroscopy 4

3 Highly Charged Ions $\quad 9$

3.1 Atomic physics of few-electron highly charged ions . . . . . . . . . 9 9

3.1 .1 Hydrogenlike ions ... . . . . . . . . . . . . . 11

3.1 .2 Heliumlike ions ................. 12

3.1.3 Electron-ion collision processes ............. 14

3.2 Production of highly charged ions . . . . . . . . . . . . . 15

4 The Livermore Electron Beam Ion Trap Facility 17

4.1 Electron beam optics in an EBIT . . . . . . . . . . . . . 18

4.1.1 Electric fields - electron beam production and acceleration . 18

4.1.1.1 Electron gun ................ 19

4.1.1.2 Trap and Collector . . . . . . . . . . . . 21

4.1.2 Magnetic fields .................... . . 22

4.1.3 Electron beam energy .................. 24

4.1.3.1 Beam radius ................ 24

4.1.3.2 Electron density .............. 30

4.1.3.3 Space charge ................ . . 33

4.1.3.4 Radial electron temperature . . . . . . . . . 43

4.1.3.5 Velocity profile ............... 45

4.2 Injection systems . . . . . . . . . . . . . . . . 53

4.2 .1 Gas injector system . . . . . . . . . . . . . 53

4.2 .2 Metal Vapor Vacuum Arc (MeVVA) . . . . . . . . . . 58

4.2 .3 Wire probe ..................... 61

4.3 Vacuum and Cryogenics .................. 61 
4.4 Operation . . . . . . . . . . . . . . . . 63

4.5 EBIT versus SuperEBIT $\ldots \ldots \ldots \ldots \ldots$

4.6 Other EBIT facilities . . . . . . . . . . . . . . . 65

$\begin{array}{lll}5 & \mathrm{X} \text {-ray Spectrometers } & 67\end{array}$

5.1 Germanium detectors . . . . . . . . . . . . . . 67

5.1 .1 Detector efficiency . . . . . . . . . . . . 68

5.1.2 Detector resolution and apparatus profile . . . . . . . 73

5.2 High-resolution crystal spectrometer . . . . . . . . . . . 77

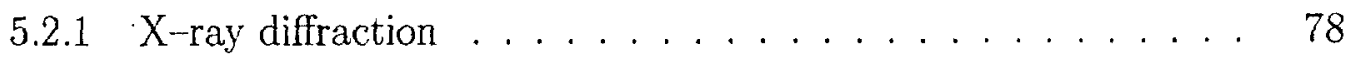

5.2.2 Reflection-type spectrometer - von Hámos geometry . . . . 79

5.2.2.1 Position-sensitive proportional counter . . . . . 84

5.2.3 Transmission-type spectrometer - DuMond geometry . . . 90

5.2.3.1 Resolving Power . . . . . . . . . . . . . 93

6 Spectroscopy on heliumlike krypton, $\mathrm{Kr}^{34+} \quad 105$

6.1 Measurements of the $\mathrm{n}=2 \rightarrow 1$ transition energies of heliumlike krypton 106

6.1 .1 Experimental setup . . . . . . . . . . . 106

6.1.1.1 Resolving power of the von Hámos spectrometer . . . 109

6.1 .2 Measurements . . . . . . . . . . . . . . 110

6.1.2.1 Inter-order calibration . . . . . . . . . . . 113

6.1.2.2 Results and brief discussion . . . . . . . . . 115

6.2 Dielectronic recombination lines in the K-shell spectra of highly charged

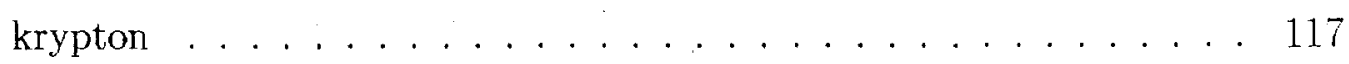

6.2.1 Experimental setup and measurement . . . . . . . 118

7 The $\mathrm{K} \alpha$ emission of heliumlike $\mathrm{Xe}^{52+}$ and hydrogenlike $\mathrm{Xe}^{53+} \quad 123$

7.1 Transmission-type crystal spectrometer measurements $\ldots . . . . .125$

$7: 1.1$ Experimental setup . . . . . . . . . . . . . 125

7.1 .2 Measurements . . . . . . . . . . . . . . . 132 
7.1.3 Electron-impact excitation cross sections . . . . . . . . . . 142 7.1.3.1 Charge balance . . . . . . . . . . . . . . . . . 144

7.1.3.2 Level-specific measurement of the xenon $\mathrm{K} \alpha$ spectrum 149 7.1.3.3 Measured cross sections . . . . . . . . . . . . . 151

7.1.4 Determination of the wavelengths . . . . . . . . . . . 169

7.2 Measurement of the $\mathrm{Ly}-\alpha_{1}$ transition energy in hydrogenlike Xe $\mathrm{e}^{53+} \cdot 175$

7.2.1 Experimental setup and measurement . . . . . . . . 176

7.2 .2 Analysis and calibration . . . . . . . . . . 177

7.2 .3 Discussion . . . . . . . . . . . . . . . . . . . 185

7.3 Conclusion . . . . . . . . . . . . . . . . . . 187

8 Summary 189

8.1 Results . . . . . . . . . . . . . . . . . . . . . . . . . . . . . . 189

8.2 Suggestions for future research . . . . . . . . . . . . . . . . . . 192

8.2 .1 Instrumental improvements $\ldots \ldots \ldots \ldots$

A Table of physical constants 194

B Velocity of electrons accelerated by an electrostatic field 195 


\section{List of Figures}

2.1 Spectral resolving power required for ion-temperature diagnostic . . . 8

3.1 Sketch of some electron-ion collision processes . . . . . . . . . . . . . 14

4.1 Schematic outline of the main features of EBIT $\ldots \ldots \ldots \ldots$

4.2 Electron Gun . . . . . . . . . . . . . . . . . . . . . . . . . 19

4.3 Drift tubes - geometry and axial potential . . . . . . . . . 23

4.4 Beam Radius . . . . . . . . . . . . . . . . . . . . . . . . . . . . . . . 29

4.5 Space Charge Potential . . . . . . . . . . . . . . . . . 37

4.6 Sketch of the electron beam passing through drift tubes of different radii 38

4.7 Space charge potential in axial direction $\ldots \ldots \ldots \ldots$

4.8 Axial space charge potential $-3 \mathrm{D}$ plot $\ldots \ldots \ldots \ldots$

4.9 Measurement of the average effective radial space charge potential in EBIT . . . . . . . . . . . . . . . . . . 42

4.10 Cyclotron and magnetron motion of the electrons $\ldots \ldots \ldots$

4.11 Axial velocity distribution along a cross section of the electron beam 50

4.12 Radial dependence of the transverse kinetic and potential energy of an electron-beam electron . . . . . . . . . . . . . . . . 51

4.13 Radial dependence of the kinetic energy of an electron-beam electron in the trap region . . . . . . . . . . . . . . . . . . . 52

4.14 Geometric properties of the gas injection system . . . . . . . 56

4.15 Potential along the electron beam axis during injection and ionization 60

5.1 Mass attenuation for beryllium and germanium . . . . . . . . 72

5.2 Efficiency of germanium detectors $\ldots \ldots \ldots \ldots \ldots . \ldots 74$

5.3 Intrinsic resolution and resolving power of the germanium detectors . 76

5.4 Implementation of the cylindrically bent crystal in the von Hámos geometry . . . . . . . . . . . . . . . . . . 81

5.5 Typical von Hámos spectrometer setup at the EBIT facility . . . . . 82

5.6 Imaging properties of the von Hámos geometry $\ldots \ldots \ldots \ldots$ 
5.7 Spatial resolution, linearity, and uniformity of the gas-filled position sensitive detector response . . . . . . . . . . . . . . . . . 91

5.8 Transmission-type versus reflection-type spectrometer at small Bragg angles ........................... 93

5.9 Geometric requirements of the DuMond-type crystal spectrometer . . 94

5.10 Sketch of the geometric components important for the calculation of the transmission spectrometer's resolving power . . . . . . . . 97

5.11 X-ray energy distribution across the detector plane . . . . . . . . . 99

$5.12 \mathrm{X}$-ray energy distribution across the detector plane . . . . . . . . 100

5.13 Simulation of the transmission-type crystal spectrometer apparatus profile . . . . . . . . . . . . . . . . . . 103

5.14 High-resolution measurement of the cesium $\mathrm{K} \alpha$ radiation . . . . . . 104

6.1 Typical von Hámos spectrometer setup at the EBIT facility . . . . 107

6.2 Heliumlike krypton direct excitation spectrum and calibration spectra 112

6.3 DE and DR lines in highly charged krypton . . . . . . . . . . 119

6.4 Identification and line out of the lithiumlike krypton KLL DR lines . 121

7.1 Experimental setup for the high-resolution measurements of the $\mathrm{n}=$ $2 \rightarrow 1$ transitions in $\mathrm{Xe}^{52+}$, and $\mathrm{Xe}^{53+} \ldots \ldots \ldots 127$

7.2 Measurement of the imaging qualities of the curved transmission-type crystal . . . . . . . . . . . . . . . . . . . . . . . . 129

7.3 Spectrum of some $\mathrm{K} \alpha$ transitions of $\mathrm{Xe}^{52+}$, and $\mathrm{Xe}^{53+} \ldots \ldots \ldots 134$

7.4 Event-mode type spectrum of the xenon $\mathrm{K} \alpha$ emission obtained with the transmission-type crystal spectrometer . . . . . . . . . . . 135

7.5 Event-mode type spectrum of the x-ray emission of SuperEBIT measured with a germanium detector $\ldots \ldots \ldots \ldots \ldots$

7.6 Xenon K $\alpha$ spectrum measured with a low-resolution germanium detector 137

7.7 Comparison of different lineouts from the high-resolution spectra . . 138

7.8 Spectrum of the $\mathrm{K} \alpha$ transitions of hydrogen- and heliumlike xenon . 139 
7.9 X-ray emission from SuperEBIT filled with highly charged xenon ions 146

7.10 Schematic diagram of the main processes for the charge balance and the population of the $n=2$ levels $\ldots \ldots \ldots \ldots \ldots \ldots$

7.11 Branching ratios for the $\mathrm{n}=2 \rightarrow 1$ transitions in hydrogenlike and heliumlike xenon . . . . . . . . . . . . . . . 161

7.12 X-ray emission of highly charged xenon ions after electron capture due to charge exchange with neutral xenon $\ldots \ldots \ldots \ldots$

7.13 Population of the $\mathrm{n}=2$ levels in $\mathrm{Xe}^{52+}$ and $\mathrm{Xe}^{53+}$ due to electronimpact excitation and radiative decay . . . . . . . . . . . 165

7.14 Experimental and theoretical values for the electron impact excitation cross sections for heliumlike $\mathrm{Xe}^{52+}$ and hydrogenlike $\mathrm{X}^{53+} \ldots \ldots 168$

7.15 Sketch of the rotation mechanism of the crystal holder . . . . . . 170

7.16 Comparison between measured and theoretically predicted transition energies for the hydrogenlike and heliumlike xenon $\mathrm{K} \alpha$ lines $\ldots \ldots .174$

7.17 Rydberg series of the $\mathrm{n} \geq 2 \rightarrow 1$ transitions in highly charged xenon . 178

7.18 Calibration spectra for the xenon $L y-\alpha_{1}$ measurement $\ldots \ldots$. . . 179

7.19 Centroid positions of the recorded spectral lines over the time of the

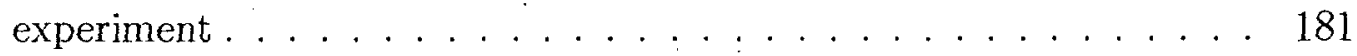

B.1 Electron velocity - relativistic versus non-relativistic . . . . . . . 196 


\section{List of Tables}

3.1 Dependence of some characteristic quantities with respect to the atomic structure and processes on the nuclear charge Z $\ldots \ldots \ldots$

4.1 Thermionic emission current . . . . . . . . . . . . . . . . . 21

4.2 Measured and calculated values of the effective electron beam radius. 31

4.3 Radial space charge Potential . . . . . . . . . . . . . . . . 36

4.4 Comparison between measured and calculated space charge potential 44

4.5 Radial dependence of some beam rotation parameters . . . . . . . . 49

4.6 Estimate of the gas injector efficiency . . . . . . . . . . . . . . 57

5.1 List of germanium detectors . . . . . . . . . . . . . . . 68

5.2 Comparison between a high- and a low-pressure position sensitive proportional counter . . . . . . . . . . . . . . . . . 89

5.3 Geometric quantities necessary for the estimation of the resolving power of a transmission-type spectrometer . . . . . . . . . . . . . . 96

6.1 Wavelengths and transition energies of the calibration lines in $\mathrm{Mn}^{24+}$, $\mathrm{Fe}^{22+}$, and $\mathrm{Fe}^{24+} \ldots \ldots \ldots \ldots \ldots \ldots \ldots$

6.2 Experimental and theoretical values for the energies of the $\mathrm{n}=2 \rightarrow 1$ transitions in heliumlike krypton . . . . . . . . . . . 117

6.3 Measured wavelengths of the lithiumlike dielectronic recombination lines 122

7.1 Ionization potential for some highly charged ions . . . . . . . . . . 123

7.2 Predicted values for the $\mathrm{K} \alpha$ transition energies for xenon . . . . . 126

7.3 Some geometric quantities associated with the transmission-type spectrometer used for the xenon measurement . . . . . . . . . . . . 130

7.4 SuperEBIT operating parameters for the xenon measurement . . . . . 133

7.5 Data acquisition time and count rates for the transmission spectrometer measurements . . . . . . . . . . . . . . . . . . . . . . . 140

7.6 Germanium detector setup parameters and xenon $\mathrm{K} \alpha \mathrm{x}$-ray flux measurement . . . . . . . . . . . . . . . . . . . 143 
7.7 Theoretical values for the radiative recombination cross sections onto highly charged xenon . . . . . . . . . . . . . 147

7.8 Fractional and absolute ion abundance of highly charged xenon ions . 149

7.9 Theoretical predictions of the electron impact excitation cross sections for the $\mathrm{n}=1 \rightarrow 2$ transitions in $\mathrm{Xe}^{52+}$ and $\mathrm{Xe}^{53+} \ldots \ldots \ldots 152$

7.10 Measured line intensities and adjustment due to the polarization sensitive Bragg diffraction . . . . . . . . . . . . . . . . 153

7.11 Measured differential and inferred total cross sections . . . . . . . 154

7.12 Estimated rates for radiative recombination, charge exchange, and electron-impact ionization of the highly charged ion ensemble in SuperEBIT . . . . . . . . . . . . . . . . . . . . 159

7.13 Charge exchange cross sections for the highly charged xenon ions . . 160

7.14 Types and statistical weights of the levels within the $n=2$ subshells . 160

7.15 Electron impact excitation cross sections for the $\mathrm{n}=1 \rightarrow 2$ transitions in heliumlike and hydrogenlike xenon . . . . . . . . 166

7.16 Experimental and theoretical values for the electron impact excitation cross sections for heliumlike $\mathrm{Xe}^{52+}$ and hydrogenlike $\mathrm{Xe}^{53+} \ldots \ldots 167$

7.17 Bragg angles, wavelengths, and transition energies for the hydrogenlike and heliumlike xenon $\mathrm{K} \alpha$ lines . . . . . . . . . . . . . . . . . 171

7.18 Comparison between measured and theoretically predicted transition energies for the hydrogenlike and heliumlike xenon K $\alpha$ lines . . . . 173

7.19 Energies of the cesium and barium $\mathrm{K} \alpha$ lines . . . . . . . . . . . . . 183

7.20 Measured xenon Ly $-\alpha_{1}$ transition energy . . . . . . . . . . . . . . . 184

A.1 Table of physical constants . . . . . . . . . . . . . . . . . . . . 194 


\section{Introduction}

The long-time goal of energy "production" by means of nuclear fusion, certainly, has been a tremendous push for the whole field of plasma physics. Substantial advances in the design and performance of the various plasma devices always depend on the availability of appropriate tools for monitoring the proposed progress. Thus, the development of diagnostic techniques and concepts has to go hand in hand with those advances, it may even be the leading hand.

High-resolution $\mathrm{x}$-ray spectroscopy, for example, has been successfully applied as a very reliable diagnostic tool for high-temperature plasmas. Based on crystal spectrometer measurements of the characteristic emission of heliumlike ions important plasma parameters, such as ion temperature, electron temperature, charge state distribution, and plasma rotation velocity can be determined. In case of very hot plasmas with electron temperatures in excess of $10 \mathrm{keV}$, as it is expected for the next generation fusion devices, characteristic $\mathrm{x}$-ray emission is provided only by heavier elements, i.e., elements that are not fully stripped in the given plasma conditions. The most likely candidates for admixture to those plasmas are the noble gases krypton and xenon. Firstly, because the introduction of gaseous media can be controlled precisely, secondly, because the chemically inert noble gases can be removed easily from the plasma device, and thirdly, because krypton and xenon have sufficiently high ionization thresholds of the $1-\mathrm{s}$ electrons and, thus, are present in the form of heliumlike ions over a broad range of high-temperature plasmas. A correct interpretation of the observed spectra, however, depends on the availability of accurate data with respect to the atomic structure and processes of the highly charged ions. The need for establishing a reliable database that includes transition energies as well as excitation, recombination, and ionization cross sections is, thus, the first step for a successful application of spectroscopic tools. This thesis is a small contribution to such a database presenting measurements regarding the characteristic $\mathrm{K}$-shell radiation of highly charged krypton and xenon. 
Starting with a very brief introduction of the methods used for determination of various plasma parameters by means of $x$-ray spectroscopy (Chapter 2), the thesis continues with a short overview of the investigated items, i.e., the highly charged ions, with respect to their presence in todays experimental devices (Chapter 3). All measurements presented in this work have been performed on the Electron-Beam-IonTrap (EBIT) of the Lawrence Livermore National Laboratory in Livermore, California. A description of the Livermore EBIT facility is given in Chapter 4 with a special focus on the properties of the electron beam. Although this device was developed only about a decade ago, it has become a corner stone in the highly-charged-ion physics community. Several benchmark measurements have been made possible by this outstanding (accelerator-free) facility. The instrumentation used for the spectroscopic measurements of the highly charged krypton and xenon emission is discussed afterwards in Chapter 5, including the low-resolution broad-band germanium detectors and the high-resolution narrow-band crystal spectrometers of von Hámos and DuMond geometry. The position-sensitive $\mathrm{x}$-ray detector used in context with the von Hámos spectrometer is mentioned in this chapter, too. Chapter 6 presents the measurements of the $n=2 \rightarrow 1$ transitions in highly charged krypton utilizing the von Hámos crystal spectrometer. In particular, the wavelengths of the heliumlike transitions and the innershell excited lithiumlike transitions have been determined. Investigation of the dielectronic recombination resonances involving $\mathrm{K}$-shell electron excitation lead to the identification of the lithiumlike satellite lines in the heliumlike krypton spectrum. Wavelength determinations are also part of the measurements of heliumlike and hydrogenlike xenon discussed in Chapter 7. The main focus of this chapter, however, is devoted to the determination of the electron-excitation cross sections of the $n=2 \rightarrow 1$ transitions. This measurement required the implementation of a high-resolution crystal spectrometer in the hard $x$-ray regime with the special requirement of high efficiency due to the low $\mathrm{x}$-ray flux provided by EBIT.

The present work demonstrates the feasibility of transferring $x$-ray spectroscopical 
plasma diagnostics to the next-generation plasma devices, which might enter temperature regimes for which no hard $\mathrm{x}$-ray instrumentation has yet been developed. Besides the instrumental component the present work also includes measurements that benchmark the current understanding of the atomic structure and processes needed for successful application of the spectra to high-temperature plasmas. 


\section{Plasma diagnostics based on x-ray spectroscopy}

The parameters of interest to plasma diagnostics are the particle densities, charge state distributions, level population densities, the kinetic energies of the particles, and the radiative properties of the plasma. Generally, all these parameters show a time dependence and a spatial distribution. Depending on the state of equilibrium a more or less extensive set of quantities is needed for the characterization of the investigated plasma, i.e., the determination of the plasma parameters.

Various methods are applied for measuring the desired quantities (e.g., $\{1,2 \mid$ ) which, basically, could be divided into intrusive and non-intrusive methods. The latter is clearly the preferable group since the object of investigation is undisturbed by the measurement. Spectroscopic diagnostic of the emitted radiation of the plasma is such a non-intrusive method [3]. In principle, there are three kinds of electronic transitions which contribute to the radiative appearance of the plasma:

- free-free transitions, where some part of the kinetic energy of the electron is converted into a photon; These transitions produce a continuous spectrum, the Bremsstrahlungskontinuum. The total kinetic energy represents the upper limit with respect to the photon energy.

- the free-bound transitions, which are indicators of a recombination process. These transitions yield characteristic edges in the spectra due to the characteristic binding energy which together with the initial kinetic energy of the electron is released in form of a photon. The binding energy is, thus, the lower limit of the characteristic edge.

- bound-bound transitions, which are the origin of the characteristic lines in the emitted radiation.

The bound-bound transitions contain most information with respect to the plasma parameters. The energy of the characteristic spectral lines, allows the identification 
of the elements in the plasma, the intensity is proportional to the particle density and the population density of the upper level, which is a measure of the excitation stage. The relative intensity of lines emitted by different ion species leads to the charge state distribution, and the line intensity ratio of lines emitted by the same ion species is sensitive with respect to the electron density. A very important spectral property is the line profile, which carries information about the kinetic energy of the ions ${ }^{2}$ and, thus, is used for the determination of the ion temperature. However, a correct interpretation of the profile has to account for the opacity of the plasma. Although several models have been developed considering significant re-absorption of the emitted radiation in the plasma $|4|$, applying spectroscopic plasma diagnostic tools to optically thin media yields a much higher accuracy with respect to the inferred plasma parameter. Therefore, it is preferable to utilize the high-energy regime of the electromagnetic radiation, i.e., the $\mathrm{x}$-ray radiation, because most plasmas are optically thin with respect to high-energy photons.

Since the deduction of the plasma parameters depends on line specific quantities, such as the profile and the intensity of the observed spectral line, a high resolving power of the spectrometer is of utmost importance for spectroscopic measurements. The need for well separated lines also favors the investigation of spectra emitted by few-electron ions. The ion species of choice utilizing $\mathrm{x}$-ray spectroscopy are the members of the heliumlike isoelectronic sequence due to

- the simplicity of the spectrum emitted by heliumlike ions;

- the high fractional abundance of the heliumlike ion species achievable over a large (electron-)temperature interval due to the large difference between the binding energies of the lithium- and heliumlike ground-state electron; The fractional abundance is also the reason for not focusing onto the hydrogenlike emission although spectra emitted by hydrogenlike ions would be best suited

\footnotetext{
${ }^{2}$ For the sake of simplicity only ions are addressed, which is justified when speaking about the very high-temperature plasmas.
} 
for line-shape characteristic investigations because of the advanced theoretical understanding.

- the requirement of optical thinness with respect to the characteristic emission, which makes $\mathrm{K}$-shell transitions favorable;

Usually, the spectroscopic investigation of plasmas in fusion devices was based on the characteristic emission of impurities intrinsic to the plasma devices. Advances with respect to the reduction of impurities, however, require the deliberate introduction of trace "impurities". The use of such seed elements has, actually, become the preferred method because it offers control of the type of element and the amount of material that should be admixed into the plasma. Thus, the element can be chosen, that will reach the heliumlike charge state in appreciable abundance at the given plasma conditions. The freedom of choice regarding the element type also allows the extension of spectroscopic plasma diagnostic tools to the next generation tokamaks, which may have electron temperatures in excess of $30 \mathrm{keV}$. Thus, reliable measurements of the central ion temperature in such hot plasmas require trace amounts of high- $\mathrm{Z}$ material in the plasma core region - prime candidates are krypton and xenon. The characteristic radiation of high-Z elements, however, is part of the hard $\mathrm{x}$-ray regime and successful implementation of the diagnostic tools depends on the development of spectroscopic instrumentation applicable to this photon energy.

The ion temperature diagnostic, for example, is based on the measurement of the Doppler broadening of the spectral line. The measured line profile also contains the apparatus profile ${ }^{3}$ and the Doppler profile has to be derived by means of deconvolution. Calculating the Doppler broadening for a spectral line $\Delta E_{D}$ an estimate can be given for the lower limit of the resolving power of the spectrometer $\frac{E}{\Delta E}$ so that the deconvolution can be performed quite comfortably, i.e.,

$$
\Delta E_{D}=E_{h \nu} \sqrt{\frac{8 \ln (2) k_{B} T}{m c_{0}^{2}}}
$$

${ }^{3}$ All other line broadening mechanisms are neglected for this estimate. 


$$
\underset{\Delta E}{E}=\sqrt{\frac{E_{m}}{8 \ln (2) E_{t h}}}
$$

where $E_{m}=m c_{0}{ }^{2}$ is the total energy of the ion, $m$ the ion mass, $c_{0}$ the speed of light in vacuum, and $E_{t h}=k_{B} T$ is the thermal energy of the ion, $k_{B}$ the Boltzmann constant, and $T$ the ion temperature ${ }^{4}$. In Figure 2.1 this lower limit of the spectral resolving power is displayed in form of a contour plot, including the binding energies of the ground state electron in lithiumlike and hydrogenlike ions. The plot shows that spectral resolving power of 1500 allows the detection of 5-keV hot krypton ions or 7$\mathrm{keV}$ hot xenon ions. The advantage of using xenon is only apparent when extremely high temperatures are present, i.e., temperatures sufficient to ionize hydrogenlike krypton $(\approx 18 \mathrm{keV})$. So far, the observation of the hard $\mathrm{x}$-ray emission of highly charged ions has been limited to low-resolution spectrometers with resolving powers of approximately 100, e.g., germanium detectors. In Chapter 5 a transmission-type crystal spectrometer is introduced capable of high-resolution measurements in the hard $\mathrm{x}$-ray regime. The measurements performed with this spectrometer (see Chapter 7) demonstrated an improvement of more than an order of magnitude with respect to the resolving power of germanium detectors and, thus, the feasibility of the implementation of spectroscopic diagnostic tools for the ion temperature determination in very high-temperature plasmas.

\footnotetext{
${ }^{4}$ It is common practice in the field of plasma physics to express temperatures in energy units and, therefore using the symbol $T$ instead of $E_{t h}$. Correctly, one should always use the expression $k_{B} T$, where $k_{B}$ is the Boltzmann constant: $\frac{1}{k_{B}}=11604.448(99) \frac{\mathrm{K}}{\mathrm{eV}}$.
} 


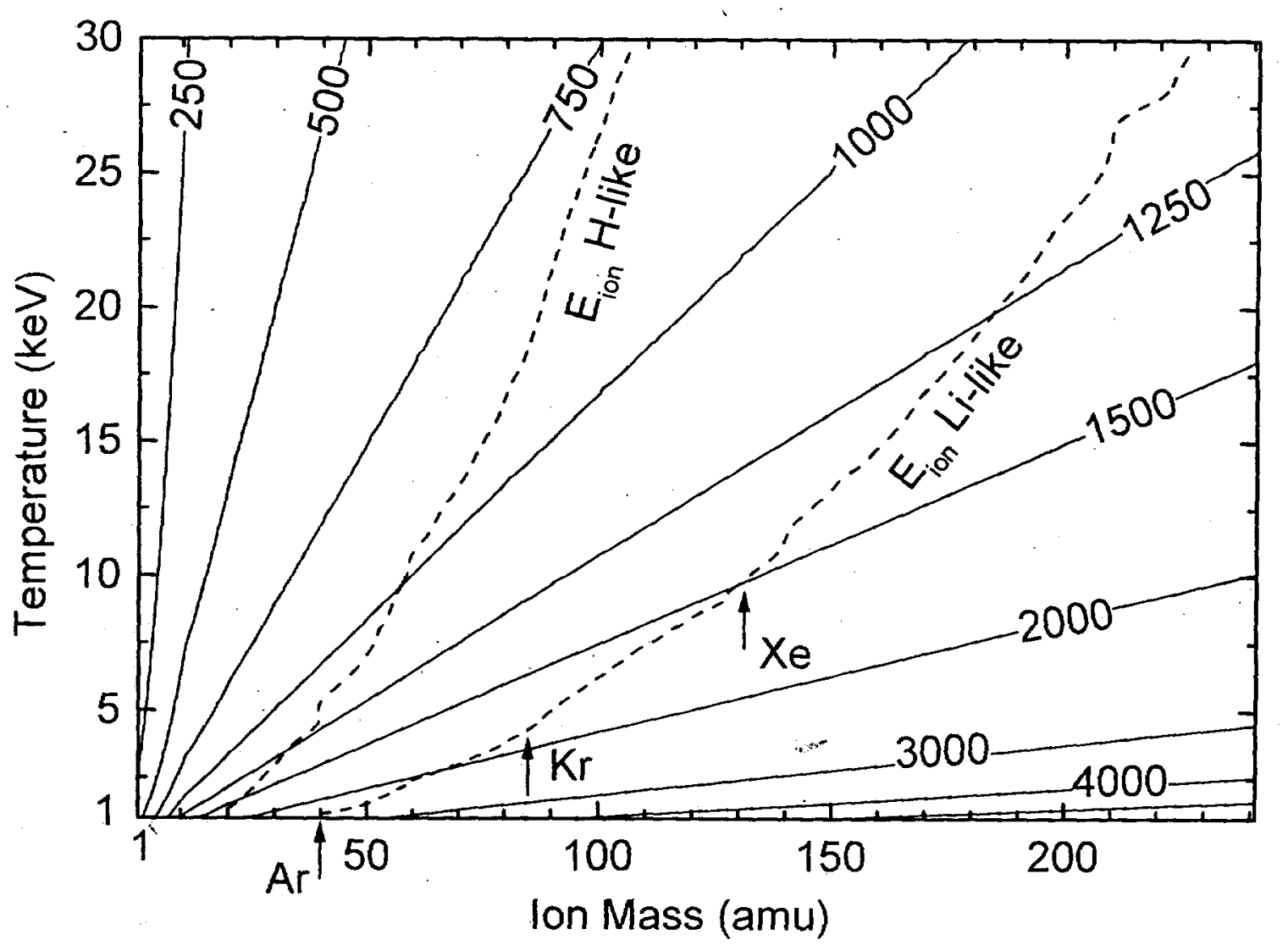

Figure 2.1: Spectral resolving power required for ion-temperature diagnostic. The values are calculated using the Doppler width expected for a given ion temperature and serve as a lower limit for the resolving power at which the deconvolution of apparatus and Doppler profile can be performed quite comfortably. The dashed lines mark the energies necessary to remove the lithiumlike ground-state electron ( $E_{\text {ion }} \mathrm{Li}$-like), thus, producing heliumlike ions, and the hydrogenlike ground-state electron ( $E_{\text {ion }} \mathrm{H}$-like), thus, fully stripping the ion. The ion mass represents the average of the naturally occuring isotope abundance. 


\section{Highly Charged Ions}

A definition of highly charged ions is given in Ref. [5], namely, that ions should be considered highly charged if the number of the remaining electrons is less than half the number of protons in the nucleus. According to this definition the first experimental evidence of highly charged ions by spectroscopic means was obtained by Edlén and Ericson in 1930, observing the Lyman series of hydrogenlike lithium, $\mathrm{Li}^{2+}$ in a spark source [6]. The emission spectra of various highly charged ions have been observed in astrophysical plasmas, e.g. the solar spectra $|7,8,9|$, and several laboratory plasmas, such as tokamak plasmas $\{3,10 \mid$, laser produced plasmas $|11,12|$, and $\mathrm{Z}$ pinches $|13|$, since. Besides their application with respect to plasma diagnostics, spectra of highly charged ions are also a subject of intense research interest from the atomic physics point of view. Especially the few-electron highly charged ions, e.g., the isoelectronic species of hydrogen, helium, and lithium, provide an ideal setting for testing approaches to solve the many-body problem.

\subsection{Atomic physics of few-electron highly charged ions}

Already the spectroscopic investigation of hydrogenlike helium, $\mathrm{He}^{+}$by Fowler $[14$ ] and Paschen $|15|$ has proven a successful guidance of theory demonstrating the feasibility of the Bohr model. The first experimental evidence of ionic line emission in the $\mathrm{x}$-ray regime by Siegbahn and Stenström [16] and of spectra from multiply charged ions by Paschen |17|, and Fowler |18| lead to the discovery of the "irregulären Dubletts" $\mid 19,20,21\}$, and nourished the development of the theory of the finestructure in the atomic spectra $[22,23,24,25,26]$.

Driven by a steady increase in the precision of the experimentally achieved values, the theoretical treatment of the atomic structure and processes becomes more and more detailed, and accounts, for example, for relativistic effects, quantum electrodynamical (QED) effects, such as the vacuum polarization and the self energy, magnetic 
interaction between the electrons, such as the Breit interaction, and nuclear size effects, e.g. the spatial charge distribution of the nucleus or the spatial distribution of the nuclear magnetic moment, referred to as Bohr-Weisskopf effect. Unfortunately, no analytic solution of the Schrödinger equation can be given for many-body configurations. In fact, even the analytic solution obtained for the hydrogen atom is, strictly speaking, an approximation since the Dirac energies are calculated under the assumption of a point nucleus, i.e., that the proton does not have a spatial extension and an internal structure, respectively. Systematic studies of the various contributions to the atomic structure and processes lead to the approximate scaling behavior, i.e., the dependence upon the nuclear charge $\mathrm{Z}$. A comparison of the $\mathrm{Z}$ scaling for some characteristic quantities with respect to the atomic structure and processes is given in Table 3.1 .

Table 3.1: Dependence of some characteristic quantities with respect to the atomic structure and processes on the nuclear charge $Z$. The effects with a stronger-than-quadratic dependence are enhanced in high- $\mathrm{Z}$ elements.

$\begin{array}{lc}\text { Quantity } & \mathrm{Z} \text { dependence } \\ \text { Atomic radius } & \mathrm{Z}^{-1} \\ \text { Dirac energy } & \mathrm{Z}^{2} \\ \text { Fine structure splitting } & \mathrm{Z}^{4} \\ \text { Hyperfine structure splitting } & \mathrm{Z}^{3} \\ \text { QED } & \mathrm{Z}^{4} \\ \text { Electron-impact excitation cross section } & \mathrm{Z}^{-4} \\ \text { Electron-impact ionization cross section } & \mathrm{Z}^{-4} \\ \text { Photoionization cross section } & \mathrm{Z}^{-2}\end{array}$

Due to the difference in the $Z$ dependence, high-Z elements provide a more sensitive platform for tests of some effects. QED contributions, for example, scale with higher 
power of $\mathrm{Z}$ than the Dirac energies and, thus, the effects of QED are enhanced in the spectra of the heaviest elements.

\subsubsection{Hydrogenlike ions}

The hydrogenlike isoelectronic sequence is without a doubt the theoretically most accurately described series. The currently most quoted theoretical predictions for the energy of the $\mathrm{n}=1$ and $\mathrm{n}=2$ states in all hydrogenlike ions $(1 \leq \mathrm{Z} \leq 110)$ are from Johnson and Soff $[27 \mid$. The calculations contain three parts, i.e., the Dirac Coulomb energy, the reduced mass correction, and the Lamb shift. The Lamb shift, originally defined as the energy difference between the $2 s_{1 / 2}$ and the $2 p_{1 / 2}$ level [28], includes, by convention [29], all corrections beyond the point nucleus Dirac eigenvalue except the non-relativistic reduced mass correction and contributions due to the intrinsic degrees of freedom of the nucleus, e.g., hyperfine structure. The Lamb shift values calculated by Johnson and Soff, for example, reflect the self energy, the vacuum polarization, the finite nuclear size, and the relativistic recoil correction.

The presumed accuracy of their calculated values, e.g., $2.9 \mathrm{ppm}$ for the Ly- $\alpha_{1}$ transition energy in $\mathrm{Xe}^{53+}$ or $28 \mathrm{ppm}$ for the same transition in $\mathrm{U}^{91+}$, has not been matched with any experimental methods, yet. So far, almost all experimental results agree with the theoretically predicted values within the uncertainty limits ${ }^{5}[30]$. Recent improvements in the determination of the energy levels including second-order QED corrections [31], however, have shown significant changes with respect to the values calculated by Johnson and Soff. In case of hydrogenlike uranium, for example, the energy of the 1-s level shifted by more than $50 \mathrm{ppm}$, i.e., almost twice the uncertainty that Johnson and Soff assigned to their calculated values.

Although, the goal is to "test QED", most measurements that have shown suffi-

\footnotetext{
${ }^{5}$ Actually, about one third of the measured values should disagree according to the definition of the $1-\sigma$ confidence limit. The extremely high percentage of agreement indicates a general overestimation of the uncertainty limits in the experimentally achieved values (Beiersdorfer, private communication).
} 
cient precision to be sensitive to the QED contributions revealed that for the high $\mathrm{Z}$ elements the uncertainties are dominated by the uncertainties regarding nuclear properties, e.g., the nuclear magnetic moment. Therefore, either a reevaluation of experimentally obtained nuclear data is necessary or based on the reliability of the theory of QED in the low-Z regime, the high-resolution spectroscopy of highly charged high- $\mathrm{Z}$ ions can be used as a new diagnostic tool for nuclear properties $\{32,33,34,35\}$.

\subsubsection{Heliumlike ions}

Spectra of heliumlike ions have been a subject of intense research interest, since these ions are the simplest of all multielectron systems, and therefore, are an ideal testbed for developing and improving approaches to solve the many-body problem and to account for the electron-electron interaction. Following overview is limited to the $\mathrm{n}=1$ and $\mathrm{n}=2$ levels and, thus, to the four main heliumlike $\mathrm{K} \alpha$ transitions, i.e., $1 s 2 p^{1} P_{1} \rightarrow 1 s^{2}{ }^{1} S_{0}, 1 s 2 p^{3} P_{2} \rightarrow 1 s^{2}{ }^{1} S_{0}, 1 s 2 p^{3} P_{1} \rightarrow 1 s^{2}{ }^{1} S_{0}$, and $1 s 2 s^{3} S_{1} \rightarrow 1 s^{2}{ }^{1} S_{0}$.

Out of the several theoretical predictions for the energy levels in heliumlike systems, three recently published methods are discussed briefly. The most complete compilation of the $n=1$ and $n=2$ energy levels including all elements of the helium isoelectronic sequence up to $Z=100$ is given by Drake $|36|$. The unified method which is described in that paper combines high-precision nonrelativistic variational calculations with relativistic and quantum electrodynamic corrections. The highprecision variational result essentially represents the sum of all terms of a commonly used $1 / Z$ expansion of the energy. Thus, the unified method accounts for all terms in the first order relativistic corrections regarding the Coulomb and the Breit interaction between the electrons and the mass polarization. The QED corrections in Drakes calculations represent the sum of all "known" one- and two-electron QED corrections of order $\alpha^{3} ; \alpha$ being the fine structure constant. The finite nuclear size corrections in Drake's calculations have been constructed from the one-electron shifts tabulated by Johnson and Soff $|27|$. The unified method also contains a term that 
accounts for the $j j \rightarrow L S$ recoupling transformation since the $L S$ coupling scheme is only appropriate for the low $\mathrm{Z}$ regime. The intermediate and high $-\mathrm{Z}$ regime is better described using the $j j$ coupling. A somewhat improved treatment with respect to Drake's calculations is given by Plante et al. |37|. Plante et al. present an all-order many-body-perturbation theory (MBPT) including corrections of order $\alpha^{4}$. Surprisingly, the QED effects are excluded in their "all-order" calculations. The instantaneous Breit interaction is treated as a perturbation and a frequency-dependent correction of the Breit energy is included along with the second-order effect of two Breit interactions. The mass polarization and QED corrections, however, are taken from Drake's calculation and it was shown recently by Persson et al. [31] that those $\mathrm{QED}$ values are not satisfactory for the high- $\mathrm{Z}$ regime. A third approach for predicting the $\mathrm{n}=1$ and $\mathrm{n}=2$ energy levels in heliumlike ions has been made by Cheng et al. using large-scale relativistic configuration-interaction calculations. The calculations by Cheng et al. , however, include several thousand configurations as a basis set for determination of the eigenvalues and eigenvectors of the Hamiltonian matrix. The relativistic corrections are treated similarly to the calculations by Plante et al., i.e., also accounting for a frequency dependent Breit correction. The QED corrections are from $a b$ initio calculations of the vacuum polarization and the self-energy in local central potentials that are screened by the moving electron itself $|38,39|$.

The differences between those theoretical predictions mentioned above are very small. Inferring the $\mathrm{K} \alpha$ transition energies from the theoretical energy level values, a variation of only $28 \mathrm{ppm}$ for the heliumlike krypton transitions energies is obtained and for the heliumlike xenon transitions, for example, the energy values are separated by less than $46 \mathrm{ppm}$. However, the differences increase with the atomic number $\mathrm{Z}$ and, thus, experimental results are necessary in order to guide the development of accurate theoretical approximations. 


\subsubsection{Electron-ion collision processes}

In context with the presented measurements, there are mainly three different electronion collision processes that account for the characteristic $\mathrm{x}$-ray emission, i.e., direct excitation (DE), radiative recombination (RR), and dielectronic recombination (DR). A sketch of these three processes is given in Figure 3.1. The $\mathrm{x}$-ray energies emitted
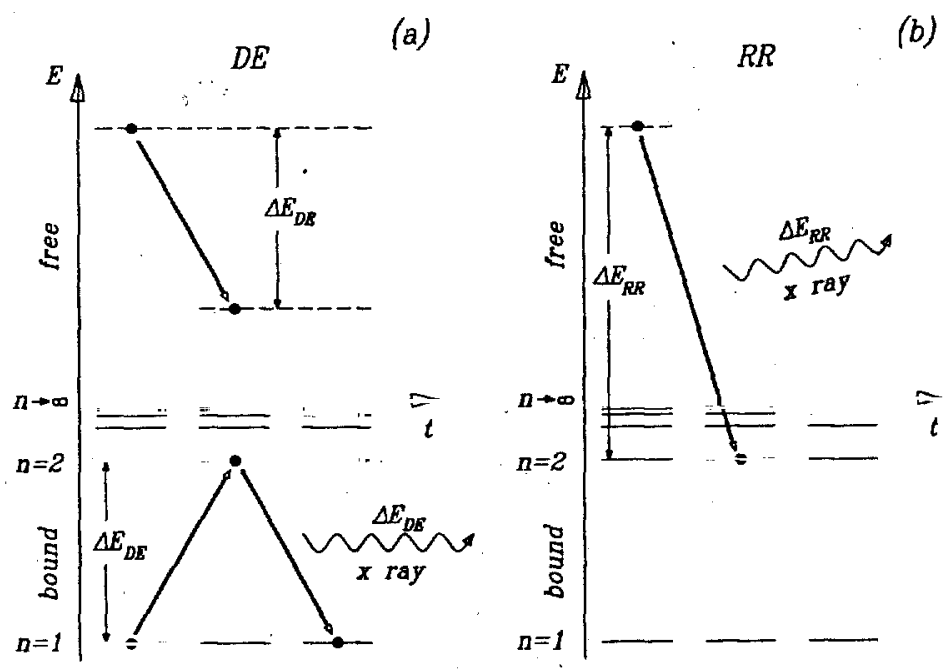

(b)

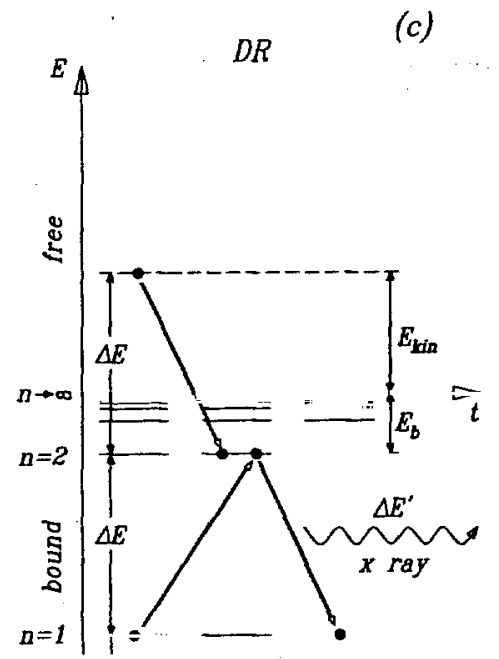

Figure 3.1: Sketch of some electron-ion collision processes in an energy-time diagram. (a) shows the direct excitation of a $\mathrm{n}=1 \rightarrow 2$ transition in the ion due to electron impact followed by radiative decay emitting the characteristic $n=2 \rightarrow 1$ line radiation. The radiative recombination process is sketched in (b). The energy of the emitted photon is the sum of the kinetic energy of the free electron and the binding energy of the level onto which the electron recombined. The dielectronic recombination shown in (c) is a resonant process, where the kinetic energy $E_{k i n}$ of the electron together with the binding energy $E_{b}$ that is released due to the recombination process match the excitation energy of a $n=1 \rightarrow 2$ transition. The characteristic line emission is slightly different from the DE process due to the recombined "spectator" electron. Note, that the energy could be also released in form of a photoelectron (autoionization).

following a recombination processes, either $\mathrm{RR}$ or $\mathrm{DR}$, are higher than the kinetic energy of the colliding electron. The energy of the emitted characteristic $x$ ray following a DR process is, however, slightly decreased due to the additional electron 
that was gained in the recombination process. The direct excited line spectrum of the $\mathrm{n}=2 \rightarrow 1$ transitions in a heliumlike ions usually contains several DR lines, i.e., $\mathrm{n}=2 \rightarrow 1$ transitions from, e.g., lithiumlike ions. Those DR lines, thus, are situated typically on the low-energy side of the DE transitions and due to the small energy difference with respect to the overall transition energies these DR lines are also known as DR satellite lines. The DR resonances are denoted according to the shells which are occupied in the excited ion, for example, KLL resonance describes the DR process where a heliumlike ion resonantly captures an electron onto the L-shell with simultaneous excitation of a heliumlike ground-state electron into the $\mathrm{L}$-shell, i.e., $1 s^{2}+\mathrm{e}^{-} \rightarrow 1 s 2 s 2 p$ or $1 s 2 p 2 p$.

The importance of DR was already discussed by Burgess $|40|$ in the context of laboratory and astrophysical plasmas. A comprehensive theoretical model allowing the assignment of all DR lines was given by Gabriel [41]. Gabriel also included a simple notation for the heliumlike and all lithiumlike KLL resonance lines. The fact, that there are four $n=2 \rightarrow 1$ transitions and 22 lithiumlike DR transitions motivated Gabriel to use the alphabet. Therefore, the four heliumlike $\mathrm{K} \alpha$ transitions are named $\mathrm{w}, \mathrm{x}, \mathrm{y}$, and $\mathrm{z}$. All other letters denote lithiumlike innershell transitions.

\subsection{Production of highly charged ions}

The occurrence of highly charged ions in various laboratory plasma devices (tokamaks, high-power laser facilities, Z-pinch devices, vacuum sparks) was already mentioned at the beginning of the chapter. Additionally, there is a set of experimental facilities especially designed for the production of highly charged ions:

- Electron Cyclotron Resonance (ECR) sources [42|; The source of the highly charged ions is a magnetically confined plasma which is resonantly, i.e., in resonance with the electron cyclotron frequency, heated by microwaves. The gyrating electrons gain sufficient kinetic energy that an ionization takes place upon collision with a neutral or ionized particle in the ECR plasma. 
- Heavy-ion storage rings - beam-foil spectroscopy [43]; A thin foil is prepared as a target for an accelerated ion beam. Upon penetration through the foil the fast ions are stripped.

- Electron Beam Ion Sources (EBIS) 444$\} ;$ An electron beam with a high current density is sufficiently accelerated for ionizing the magnetically confined ions.

- Electron Beam Ion Traps (EBIT) (see Chapter 4); In principal, the same as an EBIS but with a better and spatially shorter ion confinement. 


\section{The Livermore Electron Beam Ion Trap Facility}

The design of an Electron Beam Ion Trap (EBIT) is based on the layout of an Electron Beam Ion Source (EBIS) but it has a much shorter drift tube ${ }^{6}$ region, in particular about $2 \mathrm{~cm}$ which is more than an order of magnitude smaller than the typical length of axial confinement in an EBIS |44|. With the shorter trap region most instabilities 'of the electron beam can be avoided and the trapping time of the ions can be increased up to several minutes or even hours [45]. Due to this increase in confinement time it is possible to reach much higher charge states in an EBIT than in an EBIS. Even bare uranium ions $\left(\mathrm{U}^{92+}\right)$ have been produced and trapped at the Livermore EBIT facility [46]. Currently, the LLNL EBIT facility has two fully operational machines, EBIT, also known as EBIT II, and SuperEBIT which is a high-energy upgrade of the original EBIT I device. The main difference between EBIT and SuperEBIT is that the latter is capable of employing a higher electron beam energy. A more detailed discussion about the differences between EBIT and SuperEBIT is given below. A third machine, an intense EBIT (IEBIT) which has been designed by Marrs et al. $[47 \mid$ is currently under construction.

The major components of an EBIT are the electron beam optics (axial and radial steering elements, i.e., electrostatic and magnetic components, respectively) and the injection systems for ions, atoms, and molecules. A schematic outline of the main features is shown in Figure 4.1 (page 18). The following sections give a brief description of these components and the requirements in respect to the vacuum system and the cryogenics. Furthermore, a short introduction to the different modes of operation regarding the electron-beam energy is given and the main differences between EBIT and SuperEBIT are discussed. At the end of this chapter an attempt is made to give an overview of the status of all the EBIT facilities which have been built in the mean time emphasizing the success of this extraordinary tool for atomic physics and related, fields.

\footnotetext{
${ }^{6}$ The drift tubes are the electrodes responsible for axial confinement of the ions.
} 


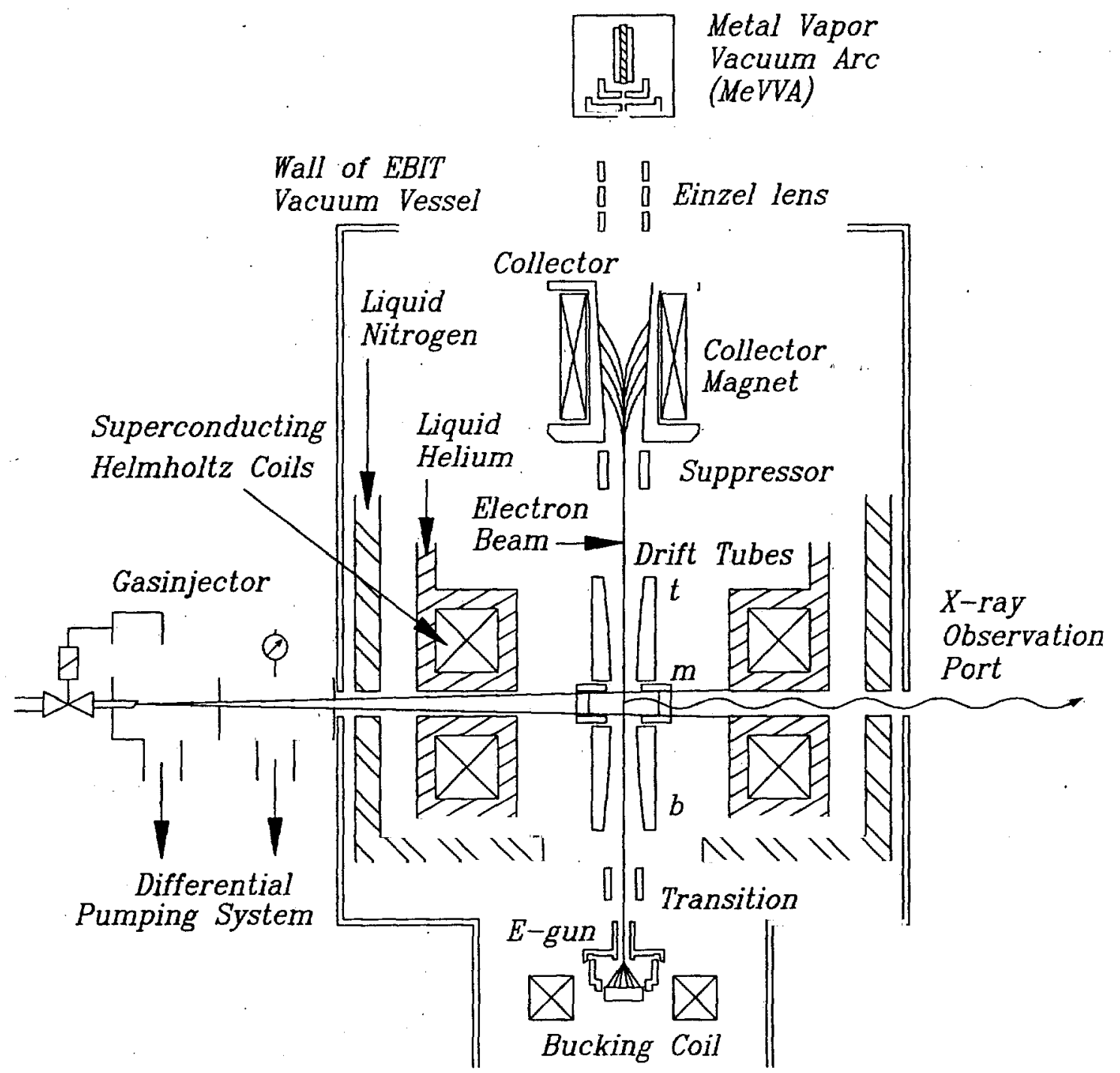

Figure 4.1: Schematic outline of the main components of EBIT. A detailed description of the labeled components can be found in the various sections of this chapter. The labels $t, m$; and $b$ denote the top, middle, and bottom drift tube.

\subsection{Electron beam optics in an EBIT}

4.1.1 Electric fields - electron beam production and acceleration 


\subsubsection{Electron gun}

The source of the electron beam is a Pierce-type electron gun ${ }^{7}$. A cross section along the optical axis is pictured in Figure 4.2. The spherical geometry of the cathode, which measures $3 \mathrm{~mm}$ in diameter, produces a focal spot of the emitting electrons which is almost an order of magnitude smaller than the diameter of the cathode itself, in particular, $360 \mu \mathrm{m} \mathrm{|49|}$. The focus electrode is necessary to compensate the "missing" electrostatic field due to the fact that the concave surface of the cathode describes only part of a sphere. The smallest aperture of the electron beam, however, can only be obtained if no magnetic field is present (see also Section 4.1.3.1). The

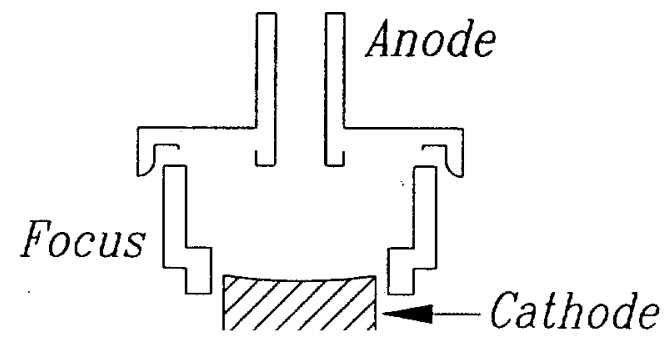

Figure 4.2: Axial cross section of the Pierce-type electron gun. The cathode is $3 \mathrm{~mm}$ in diameter and has a spherical shaped surface. The field of the focus electrode guides the emitting electron towards a $360-\mu \mathrm{m}$ wide focal spot. The electron beam current is controlled by the potential difference between Anode and Cathode, assuming space charge limited operation. The electron guns used in EBIT and SuperEBIT have a perveance of about $0.6 \mu$ perv, see eq.(4.2).

electron guns are equipped with a dispenser cathode which consists of a tungsten substrate and a barium layer. The barium diffuses into the tungsten and lowers the electron work function of the cathode material, i.e., the minimum electron energy necessary to escape from the cathode. In particular, the work function of tungsten is $\Phi_{W}=4.53 \mathrm{eV}$. For a tungsten-barium mixture it is reduced to $\Phi_{W, B a}=2.00 \mathrm{eV}$ (values taken from Ref. $|50|$ ). The drawback of using barium is the fact that is easily

\footnotetext{
${ }^{7}$ Named after J.R. Pierce, see also Reference [48]. The electron guns employed at the EBIT facility are commercially available at Raytheon Manufacturing Company, Waltham, MA. This type of electron gun was originally designed fór use in microwave tubes.
} 
oxides, which makes it necessary to keep the whole electron gun assembly under vacuum or in an inert-gas environment.

The maximum current density $j$ which can be extracted from metallic surfaces can be expressed by the Richardson-Dushman equation [51, 52],

$$
j=A T^{2} e^{-\frac{\Phi}{k T}} .
$$

$T$ is the temperature of the filament, $\Phi$ the work function, and $k$ the Boltzmann constant. In the original work by Dushman $|52|$ the constant $A$ is called the universal emission constant and is equal to $2 \pi k^{2} m_{e} e h^{-3}=60.087(1) \frac{\mathrm{A}}{\mathrm{mm}^{2} \mathrm{~K}^{2}}$, where $m_{e}$ is the electron rest mass, $e$ the elementary charge, and $h$ the Planck constant. The number in parentheses is the uncertainty in the last digit ${ }^{8}$. Later, experimental evidence was obtained that the constant $A$ also depends on the cathode material ${ }^{9}$. Table 4.1 shows the sensitivity of the maximum current based on thermionic emission in respect to the cathode temperature and the work function.

However, the saturation current density at a given cathode temperature can only be achieved if the electric field strength in front of the emitting surface is high enough to extract all the emerging electrons. Otherwise the space charge of the emitted but not extracted electrons increases the work function and, thus, lowers the saturation limit of the emission current density. In the space-charge limited regime the current density is, according to Child-Langmuir law $\{54,55,56\}$, proportional to the electron acceleration potential to the power of $\frac{3}{2}\left(j \propto U^{\frac{3}{2}}\right.$, with $U$ being the acceleration potential). Hence, for electron guns operated in the space-charge limited regime the electron-beam current only depends on the anode potential. The proportionality between the beam current $I$, and the anode potential $U$, more specifically, the potential difference between cathode and anode, is defined as the perveance,

$$
P=\frac{I}{U^{\frac{3}{2}}} \text {. }
$$

\footnotetext{
${ }^{8}$ For values and uncertainties of physical constants please see Section A (page 194)

${ }^{9}$ Sometimes, a separate factor is used to incorporate the material dependence, i.e., eq. (4.1) changes to $j=A G T^{2} e^{-\frac{Q}{k T}}$. For most metals the factor $G$ equals 2 .
} 
Table 4.1: Maximum thermionic emission current with respect to the cathode temperature $T$ and work function $\Phi$, according to eq.(4.1). For a barium impregnated tungsten cathode the constant $A$ is equal to $1 . \underset{\mathrm{mm}^{2} \mathrm{~K}^{2}}{\mathrm{~A}}$ (Ref. [53]). The cathode area of the electron guns used at the EBIT facility is $\approx 7.1 \mathrm{~mm}^{2}$ and about 3 to 4 Watt ohmic heating is applied to achieve cathode temperatures around $1300 \mathrm{~K}$ to $1400 \mathrm{~K}$.

\begin{tabular}{ccccc}
\hline \hline$T$ & $k T$ & \multicolumn{3}{c}{$I(\mathrm{~mA})$} \\
\cline { 3 - 5 } $\mathrm{K}$ & $\mathrm{eV}$ & $\Phi=2.06 \mathrm{eV}$ & $\Phi=2.01 \mathrm{eV}$ & $\Phi=1.96 \mathrm{eV}$ \\
\hline 1300 & 0.1120 & 123 & 193 & 301 \\
1350 & 0.1163 & 263 & 404 & 621 \\
1400 & 0.1206 & 532 & 805 & 1219 \\
\hline \hline
\end{tabular}

The perveance $\mathrm{P}$ depends on the geometry of the whole electron-gun assembly, the material and surface structure of the cathode, and may also include parameters specific to the whole experimental setup, e.g., type and pressure of the rest gas in the evacuated electron-gun housing. The definition of the perveance, however, is independent of any specific type of electron gun. Therefore, the perveance can be used to characterize all kinds of charged particle sources. Commonly used units for the perveance are perv, $p$, and $\frac{A}{V^{\frac{3}{2}}}\left(1\right.$ perv $\left.=1 \mathrm{p}=1 \frac{\mathrm{A}}{\mathrm{V}^{\frac{3}{2}}}\right)$. According to eq. (4.2) perveances in the order of $0.7 \cdot 10^{-6}$ perv $=0.7 \mu$ perv for EBIT and $0.6 \mu$ perv for SuperEBIT are obtained for typical operating parameters $(I=200 \mathrm{~mA}, U=4.2 \mathrm{kV}$ for EBIT and $I=250 \mathrm{~mA}, U=5.5 \mathrm{kV}$ for SuperEBIT).

\subsubsection{Trap and Collector}

After leaving the anode the electrons are accelerated towards the transition electrode and then towards the trap region, which consists of three electrodes, referred to as bottom, middle, and top drift tubes. The transition electrode, located between the 
anode and the bottom drift tube, allows the adjustment of the gradient of the accelerating field seen by the electrons and, thus, allows for a smooth "transition" from the electron gun to the trap region. The drift tubes provide the electrostatic trap for the ions in axial direction. The special geometry of the drift tubes, displayed in Figure 4.3, creates a short potential well with a very flat bottom along the spectroscopically accessible height of the trap and steep constantly sloping sides. The short length of the well is important to gain high ion densities and the flat bottom ensures an equal density distribution in axial direction. The potential outside the well has decreasing slopes in both directions due to the change in diameter of the top and bottom drift tubes. This makes it easier for very hot ions to escape which improves the so called evaporative cooling (more in Section 4.4).

After passing the top drift tube the electrons are decelerated and hit the collector. Deceleration reduces the hardness of the Bremsstrahlung and the heat load onto the collector electrode. An additional electrode, the suppressor electrode, is installed between the top drift tube and the collector. This electrode prevents secondary electrons sputtered from the collector from reaching the drift tubes. A sketch of the potential distribution along the electron-beam axis can be found in Figure 4.15, page 60 .

\subsubsection{Magnetic fields}

Along the drift tubes a high magnetic field is applied parallel to the electron beam axis, compressing the electron beam to a diameter of $50 \ldots 90 \mu \mathrm{m}\{49,58\}$. The magnetic field is provided by a set of niobium-titanium ${ }^{10}$ coils in Helmholtz geometry which are operated in superconducting mode. At a current of 160 Ampère they create a magnetic field of about 3 Tesla varying less than $0.02 \%$ in axial direction over the length of the trap. The straightness of the magnetic field lines is better than 3 mrad along the trap region, i.e., the lateral deviation is less than $75 \mu \mathrm{m}$ along the $25-\mathrm{mm}$

\footnotetext{
${ }^{10}$ The critical temperature of the alloy $\mathrm{Nb}_{0.6} \mathrm{Ti}_{0.4}$ is $T_{c}=9.8 \mathrm{~K}[59]$.
} 


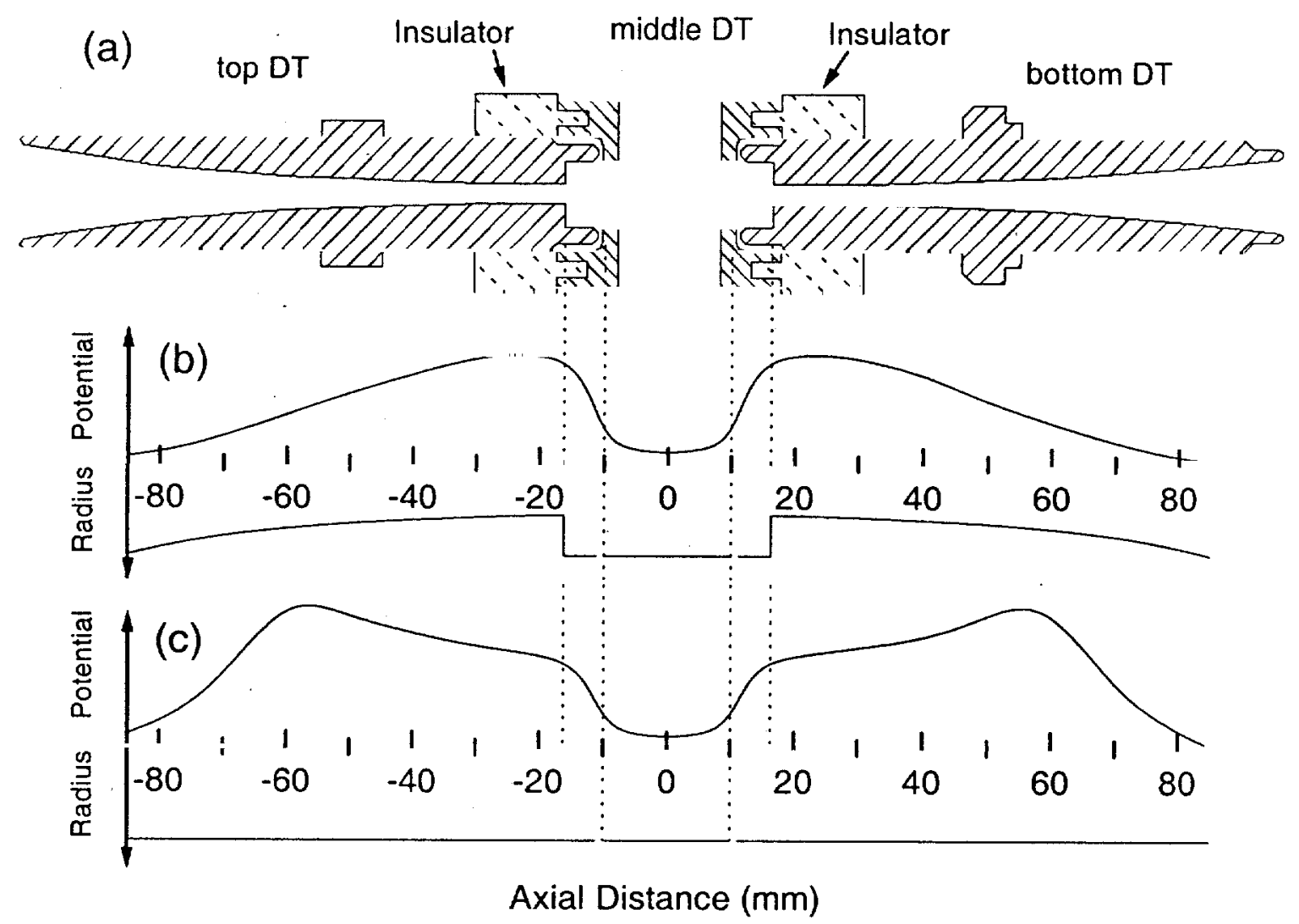

Figure 4.3: (a) Axial cross section through top, middle, and bottom drift tubes (DT). The axial potential well — calculated by Dave Vogel [57] — is displayed together with the drift tube radius for (b) the tapered drift tube design, and for (c) drift tubes of uniform cross sections, i.e., uniform radius. The gaps in the graphs visualizing the drift tube radii represent the separation between the drift tube electrodes.

long middle drift tube. However, there are two areas where no magnetic field should be present. First, the collector, where a magnetic field would prevent electrons from spreading in radial direction towards the collector electrode, and second, the electron gun, where a magnetic field would cause a spatial broadening of the electron beam. For both elements additional electromagnets are installed to compensate for the stray field from the superconducting magnets. In particular, these are the collector magnet which surrounds the collector and the bucking coil surrounding the electron-gun region. Since the position of the focal point of a Pierce-type electron gun depends on 
the anode potential, which controls the beam current, the magnetic field of the bucking coil has to be adjusted accordingly. Depending on the scope of the measurements it could be sometimes desirable that the magnetic field at the electron-gun cathode is not completely shielded. In such an environment the electrons emitted from the cathode are immediately bound by magnetic field lines and the electron beam flow approaches laminarity. When a magnetic flux is allowed at the cathode, an increase in the total magnetic field would be required to confine the beam within the same radius as in the case of the shielded cathode.

Fine tuning of the total magnetic field along the electron beam path is made possible by several steering coils. Those coils create magnetic fields perpendicular to the electron beam axis and transport coils with a magnetic field vector parallel to the electron beam.

\subsubsection{Electron beam energy}

The electron energy at the trap region is mainly determined by the potential difference between the cathode of the electron gun and the middle drift tube. A slight reduction is caused by the space charge of the electron beam itself, which besides lowering the beam energy, also causes an energy spread. The magnitude of the space charge depends on the spatial compression of the beam caused by the magnetic field. The compression also increases the thermal motion of the electrons. Knowledge about the electron beam geometry is, thus, crucial for the determination of the actual electron beam energy.

\subsubsection{Beam radius}

Several experimental studies about the dynamics of electron beams have shown that electron beam flow, especially from magnetically shielded cathodes, is highly nonlaminar $|60,61,62,63|$. The most successful theoretical approximation describing the behavior of cylindrical electron beams including the thermal motion of the electrons is 
Herrmann's so called optical theory ${ }^{11}|62|$. Assuming a Maxwellian velocity distribution for the emitted electrons, the distribution for transverse thermal velocities, i.e., parallel to the cathode surface, follows a Gaussian function with a standard deviation $\sigma_{v}=\sqrt{ } k T / m_{e}$, where $k$ is the Boltzmann constant, $T$ the temperature, and $m_{e}$ the electron mass. Herrmann divides the electrons into two different groups, non-thermal electrons, which leave the cathode perpendicular to its surface, and thermal electrons which have some momentum parallel to the surface of the cathode. The location of the non-thermal electrons is characterized by the radius $r_{e}$ describing the envelope of all non-thermal trajectories. The motion of the thermal electrons is characterized by the standard deviation $\sigma_{v}$. The overall beam envelope, i.e., the beam radius, is a superposition of non-thermal and thermal trajectories. Thus, at any given point along the beam axis the transverse cross section shows a Gaussian charge density distribution with the standard deviation $\sigma_{r}$. An effective beam radius is introduced in the form $r^{2}=r_{e}{ }^{2}+2 \sigma_{r}{ }^{2}$. This definition of effective beam radius does not represent the actual beam boundary but a rather artificial magnitude enclosing about $63.2 \%$ of the electrons. This is the fraction of the volume underneath a radial Gaussian function ${ }^{12}$ within a radius of $\sqrt{2} \sigma$. In a uniform magnetic field parallel to the beam axis and in the absence of outside electric fields, the equation of motion can be solved analytically and yields a time dependent function for the effective beam radius. At any given point along the axis the beam oscillates about an equilibrium effective beam radius $r_{0}$, which is determined by the equation

$$
\begin{gathered}
\left(\omega_{L} r_{0}^{2}\right)^{2}=K r_{0}^{2}+\frac{2 k T r_{c}{ }^{2}}{m_{e}}+\left(\omega_{L, c} r_{c}^{2}\right)^{2} \\
\text { with } \omega_{L}=\frac{e B}{2 m_{e}}, \omega_{L, c}=\begin{array}{c}
e B_{c} \\
2 m_{e}
\end{array}, \\
\text { and } K=\frac{e I}{2 \pi \epsilon_{o} m_{e} v} .
\end{gathered}
$$

\footnotetext{
${ }^{11}$ The trajectories of the electrons are assumed subject to the laws of geometrical optics of ideal optical systems.

${ }^{12}$ The volume $V$ within the radius $r$ of a 2-dimensional Gaussian function with the standard deviation $\sigma$ is: $V(r)=V_{\text {total }}\left[1-e^{-r^{2} /\left(2 \sigma^{2}\right)}\right]$.
} 
The symbol $e$ is the elementary charge, $m_{e}$ the electron mass, $k$ the Boltzmann constant, $T$ the temperature, $\epsilon_{0}$ the permittivity of vacuum, and $B$ the magnetic flux density of the uniform field. The subscript ${ }_{c}$ denotes quantities at the position of the cathode. The quantity $\omega_{L}$ is the Larmor frequency which describes the rotation of the whole beam about the beam axis $\{64,65\}$ (see also Section 4.1.3.5. Under the assumption that the axial velocity of the electrons is independent of the radial distance a solution of eq.(4.3) with respect to $r_{0}$ can be given in following form:

$$
r_{0}=\sqrt{\begin{array}{c}
m_{e} I \\
\pi \epsilon_{0} v e B^{2}
\end{array}}+\sqrt{\left(\frac{m_{e} I}{\pi \epsilon_{o} v e B^{2}}\right)^{2}}+\frac{8 k T m_{e} r_{c}^{2}}{e^{2} B^{2}}+\frac{B_{c}^{2} r_{c}^{4}}{B^{2}} .
$$

For a description of the symbols refer to eq.(4.3). In a paper by Amboss [66] demonstrating the feasibility of magnetic compression of an electron beam it was stated that Herrmann's optical theory was satisfactory only in the low-perveance regime, i.e., for electron guns with a perveance below $0.1 \mu$ perv. Therefore, Amboss developed a semiempirical theory based on a perturbation of the non-thermal paraxial-ray equation. Unfortunately, he failed to give a better suited formula for the calculation of the beam radius and, thus, uses eq.(4.4) himself ${ }^{13}$.

According to eq.(4.4) the radius $r_{0}$ is a minimum for a completely magnetically shielded cathode $\left(B_{c}=0 \mathrm{~T}\right)$. The theoretical minimum is obtained for $B_{c}=0 \mathrm{~T}$ and $T=0 \mathrm{~K}$, i.e., the perfectly-shielded non-thermal case. This minimum radius is known as the Brillouin radius $r_{B}$ and is equal to

$$
r_{B}=\sqrt{\frac{2 m_{e} I}{\pi \epsilon_{0} v e B^{2}}}
$$

In the non-relativistic regime the speed of the electrons is equal to $\sqrt{\frac{2 e V}{m_{e 0}}}, U$ being the potential difference between the cathode and the position of interest along the beam axis. The symbol $m_{e 0}$ denotes the electron rest mass. Thus; eq. (4.5) modifies

\footnotetext{
${ }^{13}$ Note, that eq. (2) in Amboss's paper [66] lacks of the electron-mass factor in the themal part of the equation and that his reference to eq. (3) in Herrmann's paper [62] should correctly state eq. (31).
} 
to

$$
\begin{aligned}
r_{B} & =\left(\frac{2 m_{e} o}{\pi \epsilon_{0} e}\right)^{1 / 2}\left(\frac{m_{e o}}{2 e}\right)^{1 / 4} \frac{I^{1 / 2}}{B U^{1 / 4}}= \\
& =K \cdot \frac{I^{1 / 2}}{B U^{1 / 4}} \\
\text { with } \quad K & =4.6686598(24) \cdot \frac{\mu \mathrm{m} \mathrm{T} \mathrm{kV}}{\mathrm{mA}^{1 / 2}} \\
& =0.01476360(1) \cdot \frac{\mathrm{cm} \mathrm{T} \mathrm{kV}^{1 / 4}}{\mathrm{~A}^{1 / 2}} .
\end{aligned}
$$

Note that at an electron energy of about $30 \mathrm{keV}$, the difference between relativistic and non-relativistic treatment of the electron velocity is already $4.4 \%$. When accounting for relativistic effects the electron speed and electron mass have to be calculated using

$$
v=c_{o} \sqrt{1-\left(\frac{m_{e O} c_{o}^{2}}{m_{e o} c_{o}^{2}+e U}\right)^{2}}
$$

(see also Appendix B).

Instead of the Brillouin radius $r_{B}$ one can also define the Brillouin field $B_{B}$ as the magnetic field necessary to compress the electron beam to a given radius $r$ assuming that the electron trajectories would follow a laminar flow model. According to Ashkin |60| the Brillouin field is of importance as a measure of decision. In particular, Ashkin shows that Herrmann's optical theory is applicable if the magnetic field applied for the beam compression is at least three times the magnitude of the Brillouin field. Modifying eq.(4.5) yields:

$$
B_{B}=\sqrt{\frac{2 m_{e} I}{\pi \epsilon_{0} v e r_{0}^{2}}} .
$$

Using following parameters, i.e., $I=130 \mathrm{~mA}, U=10 \mathrm{kV}, r_{0}=21.7 \pm 2.0 \mu \mathrm{m}, v=$ $7.75 \cdot 10^{7} \frac{\mathrm{m}}{\mathrm{s}} \approx 0.26 c_{0}$ (see eq.(4.7)) for $\mathrm{EBIT}^{14}$, and $I=230 \mathrm{~mA}, U=136 \mathrm{kV}$, $r_{0}=37.28 \pm 0.55 \mu \mathrm{m}, v=1.84 \cdot 10^{8} \frac{\mathrm{m}}{\mathrm{s}} \approx 0.61 c_{0}$ for SuperEBIT, the Brillouin field is: $B_{B}=1.2 \pm 0.1 \mathrm{~T}$ for EBIT and $B_{B}=0.68 \pm 0.01 \mathrm{~T}$ for SuperEBIT. The magnetic

\footnotetext{
${ }^{14}$ The value $r_{o}=21.7 \pm 2.0 \mu \mathrm{m}$ is taken from a beam-radius measurement which, actually, was performed at the original EBIT I device [49]. The result of that measurement, $r_{b}=27.5 \pm 2.5 \mu \mathrm{m}$, was divided by the factor $\sqrt{\ln 5}$ in order to represent the effective beam radius $r_{0}$ (see also page 30 ).
} 
flux density applied is about $3 \mathrm{~T}$. Thus, the conditions in the SuperEBIT device clearly meet - according to Ashkin - the requirements for applying Herrmann's theory. In the case of EBIT the compressing magnetic flux density is a little less than three times the magnitude of the Brillouin field. However, showing that the magnitude of the compressing field is roughly three times the Brillouin field is completely satisfactory for these estimates since the parameters beam current and acceleration voltage are vary according to the experimental requirements.

An overview of the impact of the various parameters on the beam radius is displayed in Figure 4.4 in the form of a multi-parameter plot. The calculation of the beam radius in this Figure is based on eq.(4.4) and a comparison between relativistic and non-relativistic treatment is given. The determination of the equilibrium effective beam radius defined by Herrmann's optical theory does not include any radial dependence of the axial electron velocity (see eq.(4.3)). Measurements on the velocity distribution in cylindrically symmetric electron beams, however, have demonstrated that the axial velocity of the electrons does depend on the radial distance [61,60]. A qualitative and quantitative description of the radial profile of the axial electron velocity is given in Section 4.1.3.5.

Measurements of the beam diameter at the middle drift tube were performed by imaging the beam in the $\mathrm{x}$-ray band, i.e., utilizing the $\mathrm{x}$-ray emission of the electronimpact excited ions trapped by the beam. Thus, not the actual electron beam but the beam-ion overlap was imaged. The geometry of these measurements involved a narrow slit and a position sensitive detector with a spatial resolution of about $250 \mu \mathrm{m}$. A 50 -fold magnification was obtained. The beam radii $r_{b}$ inferred from

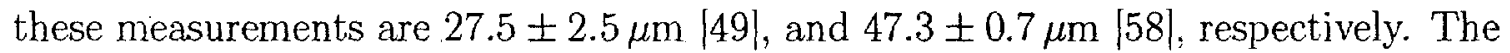
first measurement which employed a $9-\mu \mathrm{m}$ wide slit was performed on the EBIT I device (see page 17). Although, this result was obtained at EBIT I. it is used as a guidance for the magnitude of the beam radius of the current EBIT (EBIT II), since the design of both devices is very similar. The second result, i.e., $r_{b}=47.3 \pm 0.7 \mu \mathrm{m}$, 


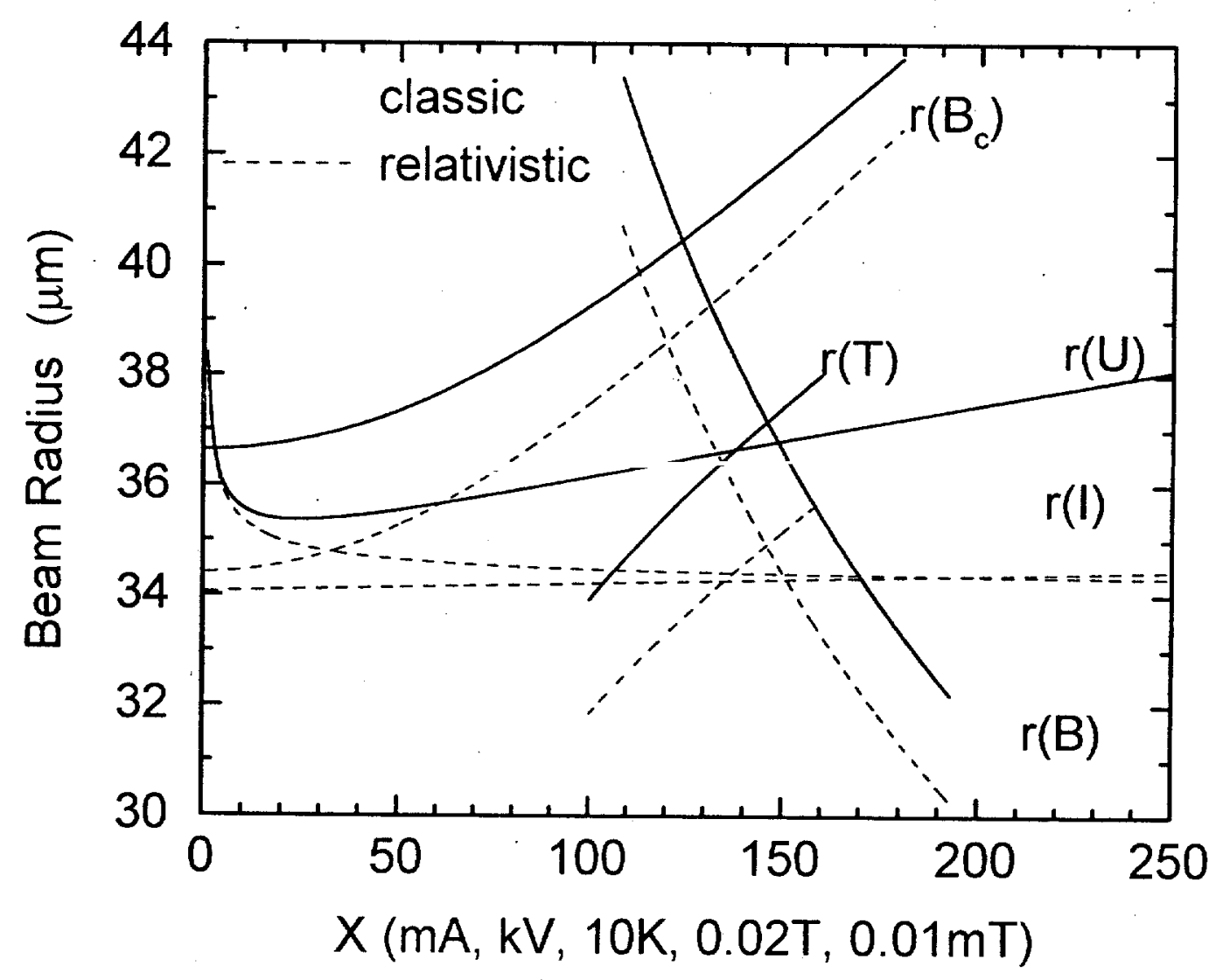

Figure 4.4: Dependence of the effective beam radius in the domain of various parameters. In particular, beam current $I$, acceleration potential $U$, magnetic flux density of the uniform magnetic field compressing the beam $B$, magnetic flux density at the cathode $B_{c}$, and temperature of the cathode $T$. While varying one parameter all other parameters were kept at following values: $I=230 \mathrm{~mA}, U=136 \mathrm{kV}, B=$ $3 \mathrm{~T}, B_{c}=0 \mathrm{~T}$, and $T=1400 \mathrm{~K}$. The beam radius is calculated using eq.(4.4). The axial velocity of the electrons is considered to be independent of the radial distance. Note that the abscissa incorporates various parameters and thus conversion factors have to be used as indicated in some cases.

was obtained at SuperEBIT using a $7-\mu \mathrm{m}$ wide slit.

The beam radii were determined taking half of the full width at one fifth of the maximum $\left(\mathrm{FW} \frac{1}{5} \mathrm{M}\right)$ of the fitted Gaussian function, which equals $2 \sqrt{2 \ln 5} \sigma_{r}$. Thus, the measured beam radius $r_{b}$ encloses $80.0 \%$ of all the electrons in the beam (see footnote 12 on page 25 ). The definition of the effective beam radius $r_{0}$ calculated 
with eq.(4.4) is, however, $r_{0}{ }^{2}=r_{e}{ }^{2}+2 \sigma_{r}^{2}$, i.e., $r_{0} \approx \sqrt{2} \sigma_{r}$. The effective beam radius, thus, only encloses $63 \%$ of all the electrons and the measured values have to be divided by the factor $\sqrt{\ln 5}$ in order to represent the effective beam radius. The measured values for the effective beam radii $\left(r_{0, \text { expt }}\right)$ are $21.7 \pm 2.0 \mu \mathrm{m}$ at EBIT I, and $37.28 \pm 0.55 \mu \mathrm{m}$ at SuperEBIT, respectively. A comparison between the measured and the calculated values is given in Table 4.2. Since neither the cathode temperature nor the magnetic flux density at the cathode have been measured, an adjustment in the calculated values can be made by variation of these two quantities. Within a temperature range of $1300 \mathrm{~K}$ to $1400 \mathrm{~K}$, agreement between the measured and the calculated values can be achieved by adjusting the magnetic flux density at the cathode from $0.4 \mathrm{mT}$ to $0.6 \mathrm{mT}$. In the case of the EBIT I measurement, however, no agreement was found by adjustment of either the temperature or the magnetic flux density. The calculated value always exceeds the measured value, even in the ideal case of no magnetic flux at the cathode surface and the lowest possible temperature which would allow electron yields of $130 \mathrm{~mA}$, i.e., $T=1250 \mathrm{~K}$ (see Section 4.1.1.1). One should keep in mind, though, that eq.(4.4) does not account for a radial dependence of the axial electron velocity and, thus, the calculated values only serve as an estimate of the effective beam radius.

\subsubsection{Electron density}

The number of electrons in a given volume of the electron beam depends on the beam current, on the compression of the beam, and the velocity of the electrons. Under the assumption of a constant beam current and beam energy within a cylindrical volume $V$, the average electron density $\bar{n}_{e}$ in this volume is given by

$$
\bar{n}_{e}=\frac{Q}{e V}=\frac{I t}{e A v \bar{t}}=\frac{I}{\pi r^{2} e v} .
$$

$Q$ denotes the whole charge in the cylindrical volume, $e$ is the elementary charge, $I$ the beam current, $r$ the radius of the cylinder, $v$ the speed of the electrons, and $t$ the time. Thus, within the measured beam radius $r_{b}$, which encloses $80 \%$ of all electrons, 
Table 4.2: Calculated values of the effective beam radius $r_{0}$ utilizing eq.(4.4). The parameters used for the calculations are listed in the top section of this Table. The calculated values should be compared to the beam-radius measurements performed at EBIT I [49], which resulted in a value of $27.5 \pm 2.5 \mu \mathrm{m}$, and SuperEBIT [58], where the measured beam radius was $47.3 \pm 0.7 \mu \mathrm{m}$. The measured values represent the radius which encloses $80 \%$ of all electrons and, thus, have to be divided by the factor $\sqrt{\ln 5}$ for comparison with the effective beam radius, which encloses $63 \%$ of all electrons. Cathode temperature and magnetic flux density at the cathode have not been measured and, thus, are adjustable parameters for the calculation.

\begin{tabular}{|c|c|c|c|c|c|c|}
\hline \multicolumn{7}{|c|}{ Parameters used for calculation } \\
\hline \multirow[b]{3}{*}{ EBIT } & $I$ & $U$ & $v_{z}$ & $B$ & $r_{c}$ & $r_{0, e x p t}$ \\
\hline & $\mathrm{mA}$ & $\mathrm{kV}$ & $c_{0}$ & $\mathrm{~T}$ & $\mathrm{~mm}$ & $\mu \mathrm{m}$ \\
\hline & $\Gamma \mathrm{I} \quad 130$ & $0 \quad 18.0$ & 0.259 & 3 & 1.5 & $21.7 \pm 2.0$ \\
\hline \multicolumn{2}{|c|}{ SuperEBIT } & 136.0 & 0.613 & 3 & 1.5 & $37.28 \pm 0.55$ \\
\hline \multicolumn{3}{|c|}{ EBIT I } & & \multicolumn{3}{|c|}{ SuperEBIT } \\
\hline$B_{c}$ & $T$ & $r_{0, \text { calc }}$ & & $B_{c}$ & $T$ & $r_{0, \text { calc }}$ \\
\hline $\mathrm{mT}$ & $\mathrm{K}$ & $\mu \mathrm{m}$ & & $\mathrm{mT}$ & $\mathrm{K}$ & $\mu \mathrm{m}$ \\
\hline 0.0 & 1250 & 34.15 & & 0.0 & 1350 & 36.48 \\
\hline 0.4 & 1400 & 35.62 & & 0.4 & 1400 & 37.26 \\
\hline 0.6 & 1300 & 35.69 & & 0.6 & 1300 & 37.20 \\
\hline
\end{tabular}

the average electron density is

$$
\bar{n}_{e}=\frac{0.8 I}{\pi r_{b}^{2} e c_{0} \sqrt{1-\left(\begin{array}{c}
m_{e O} c_{0}{ }^{2} \\
m_{e O} c 0^{2}+e U
\end{array}\right)^{2}} .}
$$

The electron speed $v$ is substituted using eq.(4.7), where $U$ denotes the potential difference between the electron-gun cathode and the middle drift tube where the 
beam radius $r_{b}$ has been measured. At a potential difference of $U=136 \mathrm{kV}$, a beam current of $I=230 \mathrm{~mA}$, and a beam radius of $r_{b}=47.3(7) \mu \mathrm{m}$, the average electron density is $\bar{n}_{e}=8.89(25) \cdot 10^{11} \mathrm{~cm}^{-3}$. Equation (4.10), however, only allows to estimate an average charge density, i.e., the charge density for a homogeneous distribution of the electrons. The actual density distribution is determined by the distribution of the transverse thermal velocities of the electrons which follows a Gaussian function (see Section 4.1.3.1). Electrons emitted from any particular point at the cathode spread out according to their transversal velocities and, thus, produce at every transverse cross section of the beam a Gaussian charge distribution. Assuming only a radial dependence of the charge density, the Gaussian electron density distribution $n_{e}$ can be written in the form

$$
n_{e}(r)=n_{e \theta} e^{-\frac{r^{2}}{2 \sigma r^{2}}}
$$

The quantities $n_{e 0}$ and $\sigma_{r}$ are implicitly defined by the measured beam radius $r_{b}$ which encloses $80 \%$ of the total charge. In an explicit form $n_{e 0}$ and $\sigma_{r}$ are:

$$
\begin{aligned}
& \sigma_{r}^{2}=\frac{r_{b}{ }^{2}}{2 \ln 5} \\
& n_{e 0}=\frac{I \ln 5}{\pi r_{b}^{2} e v} \text {. }
\end{aligned}
$$

Comparison between the homogeneous and the Gaussian distribution shows that $n_{e o} \approx 2 \bar{n}_{e}$. In particular, given the same parameters as above the electron density in the center of the beam is $n_{e}=1.788(50) \cdot 10^{12} \mathrm{~cm}^{-3}$. Along the measured beam radius the electron density dropped to $n_{e}\left(r_{b}\right)=\frac{1}{5} n_{e 0}=3.58(10) \cdot 10^{11} \mathrm{~cm}^{-3}$, where $r_{b}=47.3(7) \mu \mathrm{m}$.

The broad range of applicable beam currents and electron energies in EBIT and SuperEBIT allows to vary the electron density in the trap region from about $10^{12} \mathrm{~cm}^{-3}$ down to $10^{9} \mathrm{~cm}^{-3}$ and, of course. less. The lower limit of $10^{9} \mathrm{~cm}^{-3}$ rather reflects a practical than technical limit, since a minimum electron density is necessary for successful competition of the electron-impact ionization with atom-ion or molecule- 
ion charge exchange processes ${ }^{15}$. The lower the electron density, the smaller the fraction of high charge states achievable in the trap, and the lower the electronimpact excitation rate for these ions. Thus, the flux of the emitted $\mathrm{x}$ rays strongly depends on the electron density in the trap. Moreover, the electron density is also the base for the space charge of the electron beam which is responsible for the radial trapping of the highly charged ions.

\subsubsection{Space charge}

Assuming stationary conditions, the electric field strength and, thus, the potential can be calculated using the law of Gauß, i.e.,

$$
\oint_{A} \vec{E} \cdot d \vec{A}=\frac{Q}{\epsilon_{0}}
$$

where $\vec{E}$ denotes the electrical field strength, $A$ a closed surface with the surface element $d \vec{A}$ pointing away from the enclosed volume, $Q$ the total electrical charge in the from $A$ enclosed volume, and $\epsilon_{0}$ is the permittivity of vacuum. A closed surface integral over a vector field can be expressed as a volume's integral over the divergence of this vector field and substituting the electric field by the gradient of the potential allows to modify eq.(4.13) into the Poisson equation. In particular,

$$
\begin{aligned}
\oint_{A} \vec{E} \cdot d \vec{A} & =\int_{V} d i v \cdot \vec{E} d V=\int_{V} \nabla \cdot \vec{E} d V \\
Q & =\int_{V} \rho d V \\
\nabla \cdot \vec{E} & =\nabla \cdot(-\nabla U)=-\nabla^{2} U \\
-\nabla^{2} U & =\Delta U=-\frac{\rho}{\epsilon_{0}},
\end{aligned}
$$

where the symbol $\Delta$ denotes the Laplace operator, $\Delta=\nabla \cdot \nabla$.

The easiest approach is to consider the beam as an infinite cylindrical column of radius $R_{c y l}$ with a homogeneous charge density. The electrical field strength for such

\footnotetext{
${ }^{15}$ Charge exchange between a highly charged ion and another ion is very unlikely in an EBIT due to the low kinetic energies of the ions and the strong Coulomb repulsion.
} 
a column is

$$
\begin{aligned}
& \vec{E}\left(|\vec{r}| \leq R_{c y l}\right)=-\frac{I r}{2 \pi \epsilon_{0} v_{z} R_{c y l}{ }^{2}} \cdot \frac{\vec{r}}{r} \\
& \vec{E}\left(|\vec{r}| \geq R_{c y l}\right)=-\frac{I}{2 \pi \epsilon_{o} v_{z} r} \cdot \frac{\vec{r}}{r} .
\end{aligned}
$$

With $I$ being the electron beam current, $r$ the radial distance, and $v_{z}$ the component of the electron velocity parallel to the beam axis. The electrical field vector points towards the center of the electron beam. Extracting the potential from these equation and choosing the drift tube wall as the point of zero potential, i.e., $U\left(R_{D T}\right)=0 \mathrm{~V}$, gives

$$
U\left(r \leq R_{c y l}\right)=\frac{I}{4 \pi \epsilon_{0} v_{z}}\left[\left(\frac{r}{R_{c y l}}\right)^{2}+\ln \left(\frac{R_{c y l}}{R_{D T}}\right)^{2}-1\right]
$$

and

$$
U\left(r \geq R_{c y l}\right)=\frac{I}{4 \pi \epsilon_{0} v_{z}} \ln \left(\frac{r}{R_{D T}}\right)^{2}
$$

as solutions for the potential inside and outside the homogeneously charged infinite cylinder. Table 4.3 lists some results for EBIT and SuperEBIT utilizing these equations and includes the parameters used, i.e., beam current, acceleration potential, and measured beam radius. The radial dependence of the space charge potential is depicted graphically in Figure 4.5. From Table 4.3 it can be seen that the potential difference between the center of the beam and the beam edge, $U_{0, R_{c y l}}$, is $15.1 \pm 3.1 \mathrm{~V}$ for EBIT and $11.2 \pm 0.7 \mathrm{~V}$ for SuperEBIT. These values represent the energy spread of those electrons which interact with the trapped ions since the beam radius measurement is based on the image of the ion-electron-beam overlap. For classic treatment of the electron velocity this beam center to beam edge space charge potential can be 
expressed in analytic form, i.e.,

$$
\begin{aligned}
U_{0, R_{c y l}}=U_{s c, c l} & =\frac{I}{4 \pi \epsilon_{0}} \sqrt{\frac{m_{e 0}}{2 e}} \frac{I}{U^{1 / 2}}=K \frac{I}{U^{1 / 2}} \\
\text { with } \quad K & =15153.598(10) \frac{\mathrm{V}^{3 / 2}}{\mathrm{~A}} \\
& =0.47919884(32) \frac{\mathrm{kV}^{3 / 2}}{\mathrm{~A}} .
\end{aligned}
$$

To include relativistic effects an additional factor, $\Delta_{v}$, is required which expresses the relative difference between the classic and relativistic treatment of the electron velocity (see Appendix B, eq.(B.6) on page 196).

$$
U_{s c, r e l}=U_{s c, c l}\left(1+\Delta_{v}\right)
$$

The increase of the beam center to beam edge space charge potential due to relativistic effects is $2.6 \%$ for an electron-beam energy of $18 \mathrm{keV}$ and $19 \%$ for an electron-beam energy of $136 \mathrm{keV}$.

However, as shown in Section (4.1.3.1) the beam profile is of Gaussian nature with respect to the charge density distribution. Solving eq.(4.13) and (4.17) using an electron density distribution according to eq.(4.11) yields

$$
\begin{aligned}
\vec{E}(\vec{r}) & =\frac{n_{e_{0}} e \sigma_{r}^{2}}{\epsilon_{0} r}\left(\mathrm{e}^{-\frac{r^{2}}{2 \sigma_{r}^{2}}}\right) \cdot \frac{\vec{r}}{r} \\
& =\frac{I}{2 \pi \epsilon_{0} v_{z} r}\left(\mathrm{e}^{-\frac{r^{2}}{r_{b}^{2}} \ln 5}-1\right) \cdot \frac{\vec{r}}{r}
\end{aligned}
$$

for the electric field strength and

$$
U(r)=\frac{I}{4 \pi \epsilon_{o} v_{z}} \sum_{m=1}^{\infty} \frac{(-\ln 5)^{m}}{m \cdot m !}\left[\left(\frac{R_{D T}^{2}}{r_{b}^{2}}\right)^{m}-\left(\frac{r^{2}}{r_{b}^{2}}\right)^{m}\right]
$$

for the radial space charge potential. The result of applying these formulae is given in

Figure 4.5. Some calculated values for the space charge potential are explicitly listed in Table 4.3. Using the Gaussian electron density distribution model the potential 
difference between the beam center and the distance $R_{c y l}$ which defines the edge of the beam for the homogenous electron density model is $19.9 \pm 2.7 \mathrm{~V}$ for EBIT and $15.0 \pm 0.6 \mathrm{~V}$ for SuperEBIT, i.e., more than $30 \%$ higher than for the homogenous model.

Table 4.3: Some calculated values for the radial space charge potential $U_{s c}$ of the electron beam using eq.(4.20), (4.21), and (4.25). The parameters used for these calculations are listed in the top section of this Table. The quantity $r_{b}$ is the measured beam radius which includes $80 \%$ of all beam electrons, and $R_{c y l}$ is the radius of the beam edge assuming a homogenous electron density distribution (cylinder model). The electron velocities $v$ were calculated using eq.(4.7) and are listed as fractions of the speed of light in vacuum $c_{0}$.

\begin{tabular}{rccccc}
\hline \hline & \multicolumn{6}{c}{ Parameters used for calculation } \\
& $I$ & $U$ & $r_{b}$ & $R_{c y l}$ & $v$ \\
& $\mathrm{~mA}$ & $\mathrm{kV}$ & $\mu \mathrm{m}$ & $\mu \mathrm{m}$ & $c_{0}$ \\
\hline EBIT & $130 \pm 1$ & $18.0 \pm 0.1$ & $27.5 \pm 2.5$ & $30.8 \pm 2.8$ & $0.2586 \pm 0.0014$ \\
SuperEBIT & $230 \pm 1$ & $136.0 \pm 0.1$ & $47.3 \pm 0.7$ & $52.9 \pm 0.8$ & $0.61337 \pm 0.00045$
\end{tabular}

\begin{tabular}{ccccc}
\multicolumn{4}{c}{ Space charge potential } \\
\hline \multicolumn{3}{c}{ EBIT } & \multicolumn{2}{c}{ SuperEBIT } \\
$r$ & $U_{s c} / \mathrm{V}$ & \multicolumn{2}{c}{$U_{s c} / \mathrm{V}$} \\
$\mathrm{mm}$ & Homogenous & Gaussian & Homogenous & Gaussian \\
0.0 & $-168.5 \pm 3.1$ & $-172.7 \pm 2.7$ & $-113.5 \pm 0.7$ & $-116.6 \pm 0.6$ \\
$r_{b}$ & $-156.5 \pm 3.1$ & $-155.1 \pm 2.7$ & $-104.5 \pm 0.7$ & $-103.6 \pm 0.6$ \\
$R_{c y l}$ & $-153.4 \pm 3.1$ & $-152.8 \pm 2.7$ & $-102.3 \pm 0.7$ & $-101.6 \pm 0.6$ \\
0.5 & $-69.4 \pm 0.8$ & $-69.4 \pm 0.6$ & $-51.8 \pm 0.5$ & $-51.7 \pm 0.4$ \\
1.5 & $-36.3 \pm 0.7$ & $-36.3 \pm 0.6$ & $-27.1 \pm 0.5$ & $-27.1 \pm 0.4$
\end{tabular}

Due to the different radii of bottom, top and middle drift tube the electron beam 


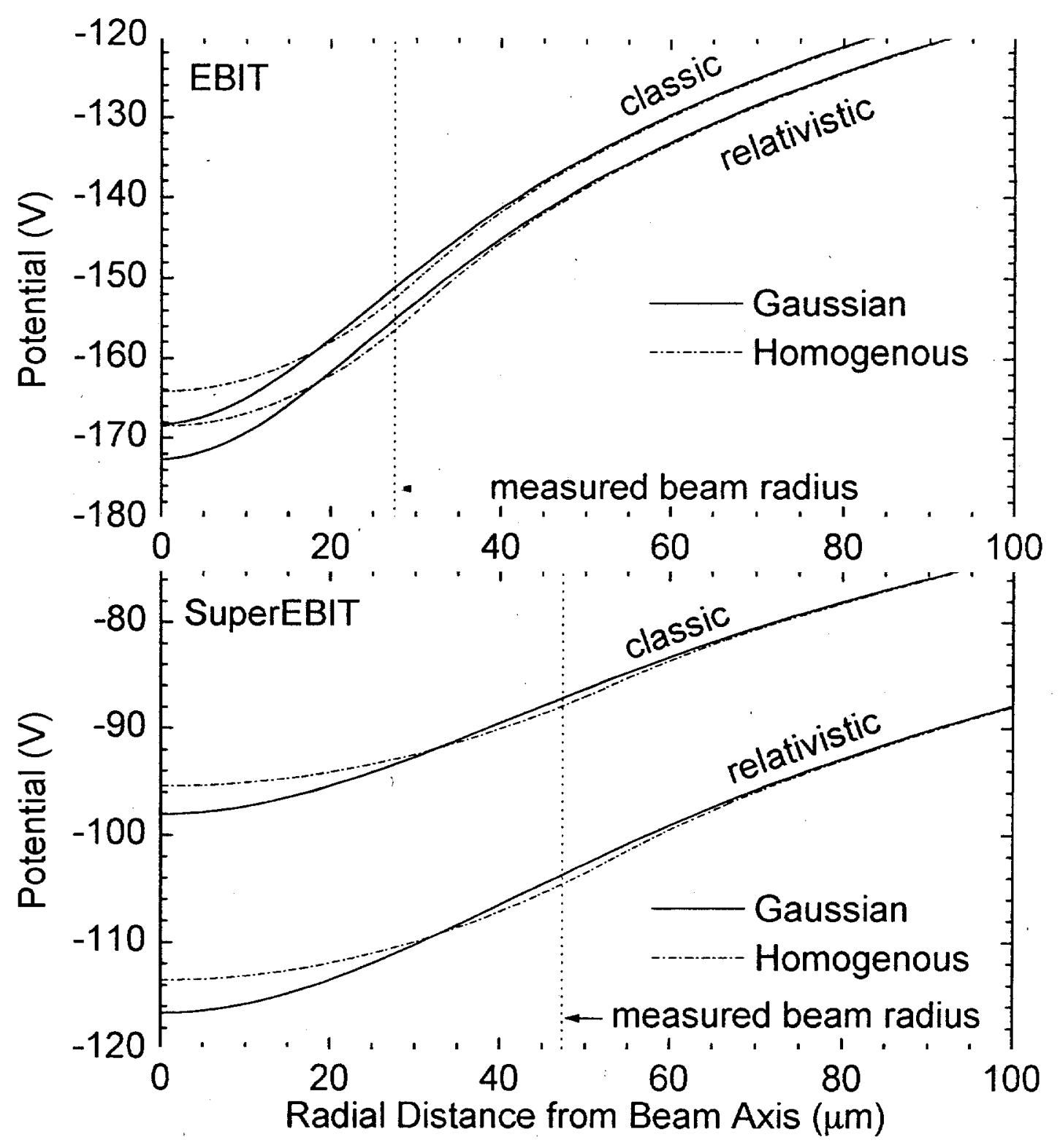

Figure 4.5: Dependence of the space charge potential of the electron beam on the radial distance from the beam axis for EBIT and SuperEBIT. The plot represents the results obtained by assuming that first, the electron beam were a homogeneously charged cylinder (see eq.(4.20 and 4.21)) - dash-dotted lines - and second, the electron beam profile were of Gaussian nature regarding the electron density (see eq.(4.25)) - solid lines. The parameters used for calculating the space charge potentials are listed in Table 4.3 (page 36).

creates a space charge potential also in axial direction. This axial space charge potential allows to trap ions axially without applying any voltage, or trapping potential to be more specific, onto the drift tubes themselves. For simplicity the following 
derivation of the axial trapping potential due to the geometrical properties of the drift tubes is based on the assumption that the beam is a homogeneously charged cylinder. Considering the electron beam surrounded by cylindrical drift tubes of different radius, as pictured in Figure 4.6. Since the potential along the edge of the electron beam has to be constant - otherwise the electrons could gain kinetic energy although no additional energy is fed into the system - the beam radius has to change when the radius of the surrounding drift tube changes. The "new" beam radius can

(a)

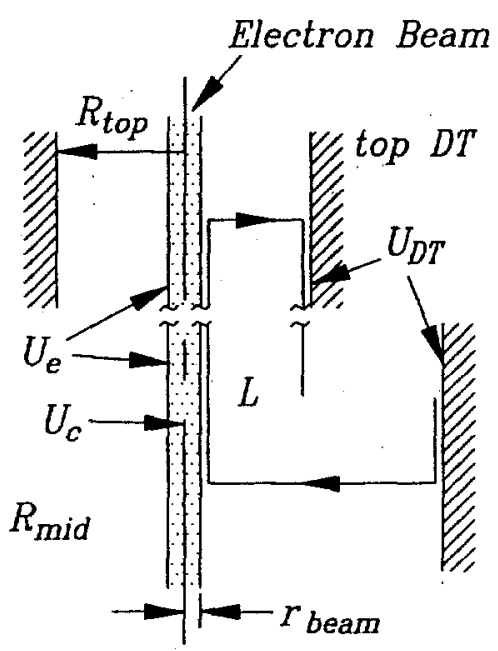

(b)

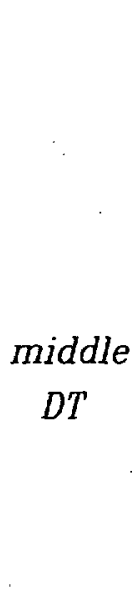

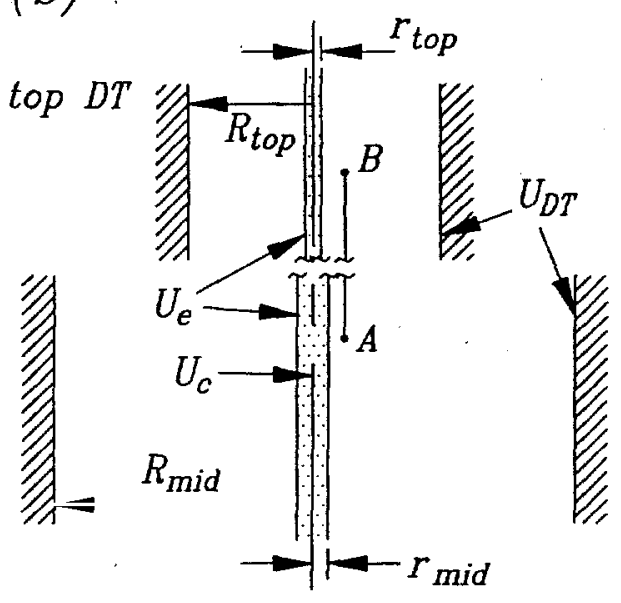

Figure 4.6: Sketch of the geometric properties for the case that the electron beam passes through drift tube sections of different radius but constant potential $U_{D T}$. The potentials at the beam edge $U_{e}$, and in the center of the beam $U_{c}$ have to be constant as discussed in the text. If the beam radius were to stay constant, (a), energy could be generated by moving a charged particle along the loop $L$ without feeding this energy into the system. Thus, the beam radius has to change, (b), which creates a potential gradient in axial direction. The potential difference between the points $A$ and $B$ depends on the radial distance.

be calculated using eq.(4.20) or eq.(4.21). For the notation refer to Figure 4.6:

$$
r_{t o p}=r_{m i d} \frac{R_{t o p}}{R_{\text {mid }}} .
$$

Moving along a path of constant radial distance, i.e., parallel to the beam axis, the 
axial space charge potential can be estimated as follows.

$$
\begin{aligned}
U\left(r \leq r_{\text {top }}\right) & =\frac{I}{4 \pi \epsilon_{0} v_{z}} r^{2}\left[\left(\frac{1}{r_{\text {top }}}\right)^{2}-\left(\frac{1}{r_{\text {mid }}}\right)^{2}\right], \\
U\left(r_{\text {top }} \leq r \leq r_{\text {mid }}\right) & =\frac{I}{4 \pi \epsilon_{o} v_{z}}\left[\ln \left(\frac{r}{r_{\text {top }}}\right)^{2}-\left(\frac{r}{r_{\text {mid }}}\right)^{2}+1\right]
\end{aligned}
$$

and

$$
U\left(r_{\text {mid }} \leq r\right)=\frac{I}{4 \pi \epsilon_{o} v_{z}} \ln \left(\frac{R_{\text {mid }}}{R_{\text {top }}}\right)^{2} \neq \mathrm{fct}(r)
$$

Thus, qualitatively, the radial dependence of the axial space charge potential is of quadratic nature from the beam center to the narrowest beam radius, shows a logarithmic dependence between narrowest and widest beam radius, and is constant outside the widest beam radius. As seen in Figure 4.3, the bottom and top drift tubes have a tapered geometry. Next to the middle drift tube the inner tube diameter is $10 \mathrm{~mm}$, after $5 \mathrm{~mm}$ in axial direction this diameter narrows abruptly to $3 \mathrm{~mm}$, and then widens smoothly to about $16.4 \mathrm{~mm}$. Thus, the difference between the space charge potential at the middle drift tube region $(10 \mathrm{~mm}$ diameter) and the top (or bottom) drift tube has been calculated as a function of the axial distance, where the narrowest part of the top (or bottom) drift tube, i.e., the 3-mm radius bottle neck, was taken as the origin with respect to the axial distance $z$. The result of these calculations of the potential difference in axial direction is shown in the Figures 4.7 and 4.8. Using the beam parameters listed in Table 4.3 the highest values for the axial space charge potential, obtained with eq.(4.29), is $36.2 \mathrm{~V}$ for EBIT and $27.0 \mathrm{~V}$ for SuperEBIT. Note, that these results can only be used as estimates of the axial potential barrier seen by the trapped ions, since the model applied, first, is not able to describe the actual region of change in the drift tube diameter, and second, does not include the effect of magnetic fields on the change of the beam radius.

In all cases, however, the radial trapping potential was modeled under the absence 


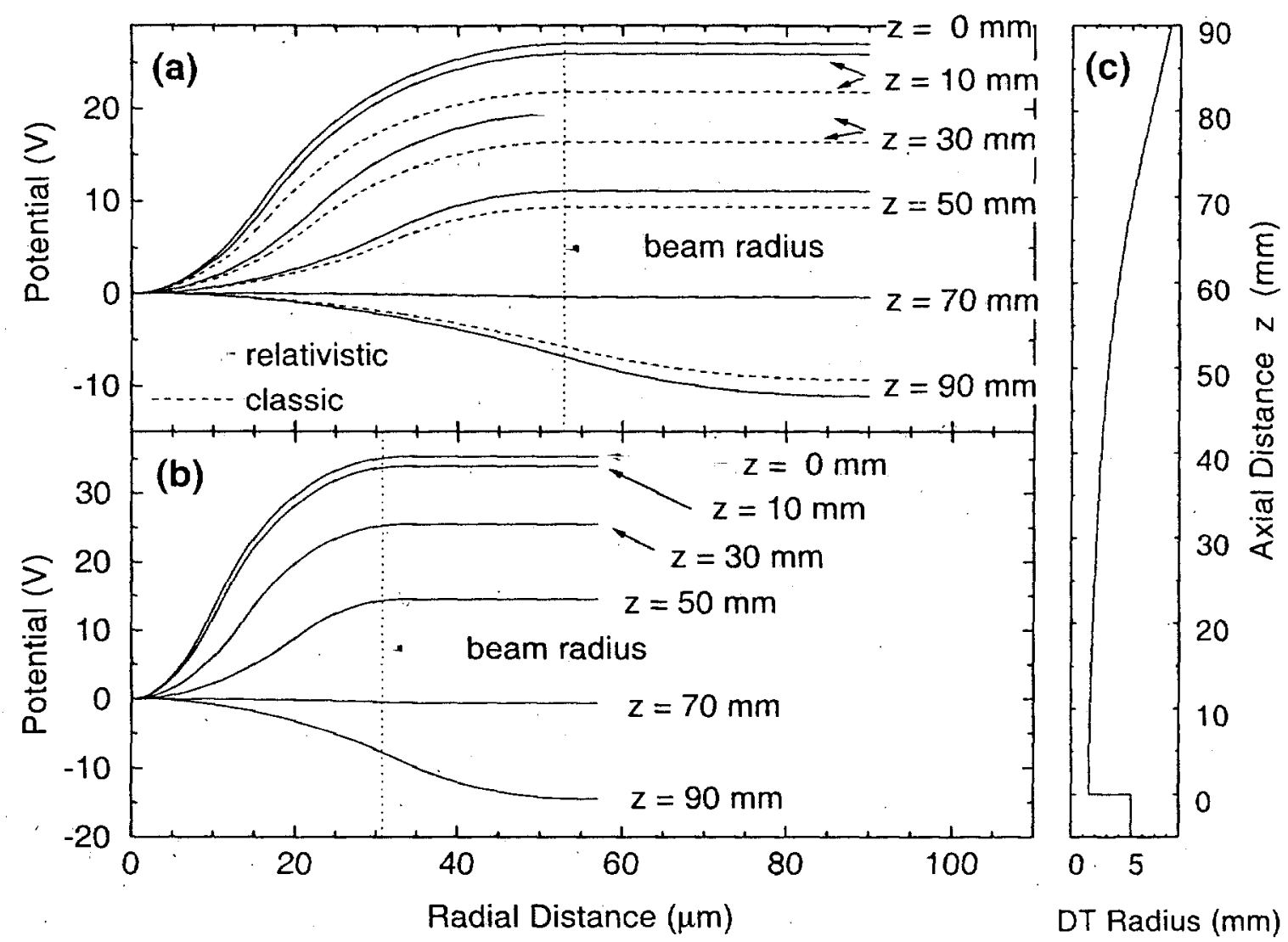

Figure 4.7: Difference between the radial space charge potential at various positions along the top (or bottom) drift tube and the radial space charge potential in the middle drift tube region for SuperEBIT (a) and EBIT (b). Note that the graphs display a potential difference and not the overall radial space charge potential! Plot (c) shows the radius of the top (or bottom) drift tube with respect to the axial distance $z$.

of any ions. The compensation of the space charge potential due to the positively charged ions can be accounted for by an additional factor $(1-f)$, where

$$
f=\frac{n_{q} q}{n_{e}}
$$

i.e., the ratio of positive to negative charges within the electron beam volume of interest. Practically, the space charge compensation factor $f$ can obtain values between zero and one depending on the operating parameters of the EBIT devices. For $f$ values bigger than one $(f>1)$, which is theoretically possible according to the definition 


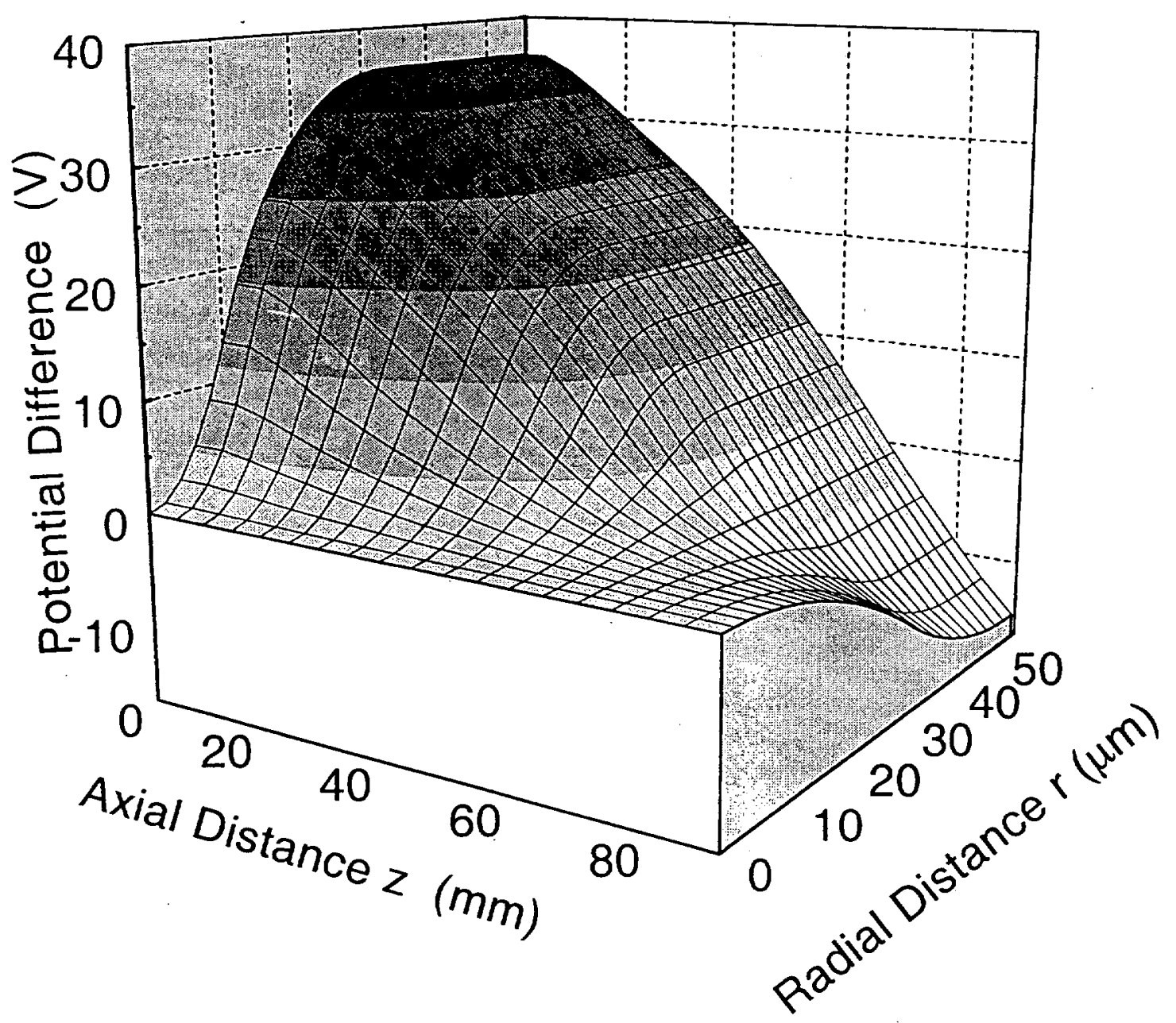

Figure 4.8: Variation of the space charge potential with respect to the radial space charge potential in the middle drift tube region. The potential differences are plotted as a function of the radial distance from the beam axis and the axial distance from the narrowest diameter of the top (or bottom) drift tube.

of $f$ in eq.(4.31), the space charge potential becomes positive and the ions leave the electron beam. Although the ions are still trapped radially by the magnetic field they are not exposed anymore to any electron-impact excitation mechanism and, thus, are lost in a spectroscopical sense.

The space charge compensation factor can be measured by observing electronbeam energy dependent resonance processes, such as dielectronic recombination (DR) 
transitions (see Section 3.1.3). Such a measurement is presented in Figure 4.9 which shows the potential difference between the electron-gun cathode and the middle drift tube necessary for achieving maximum emission intensity of a specific DR transition as a function of the electron-beam current. In particular, the DR line emission

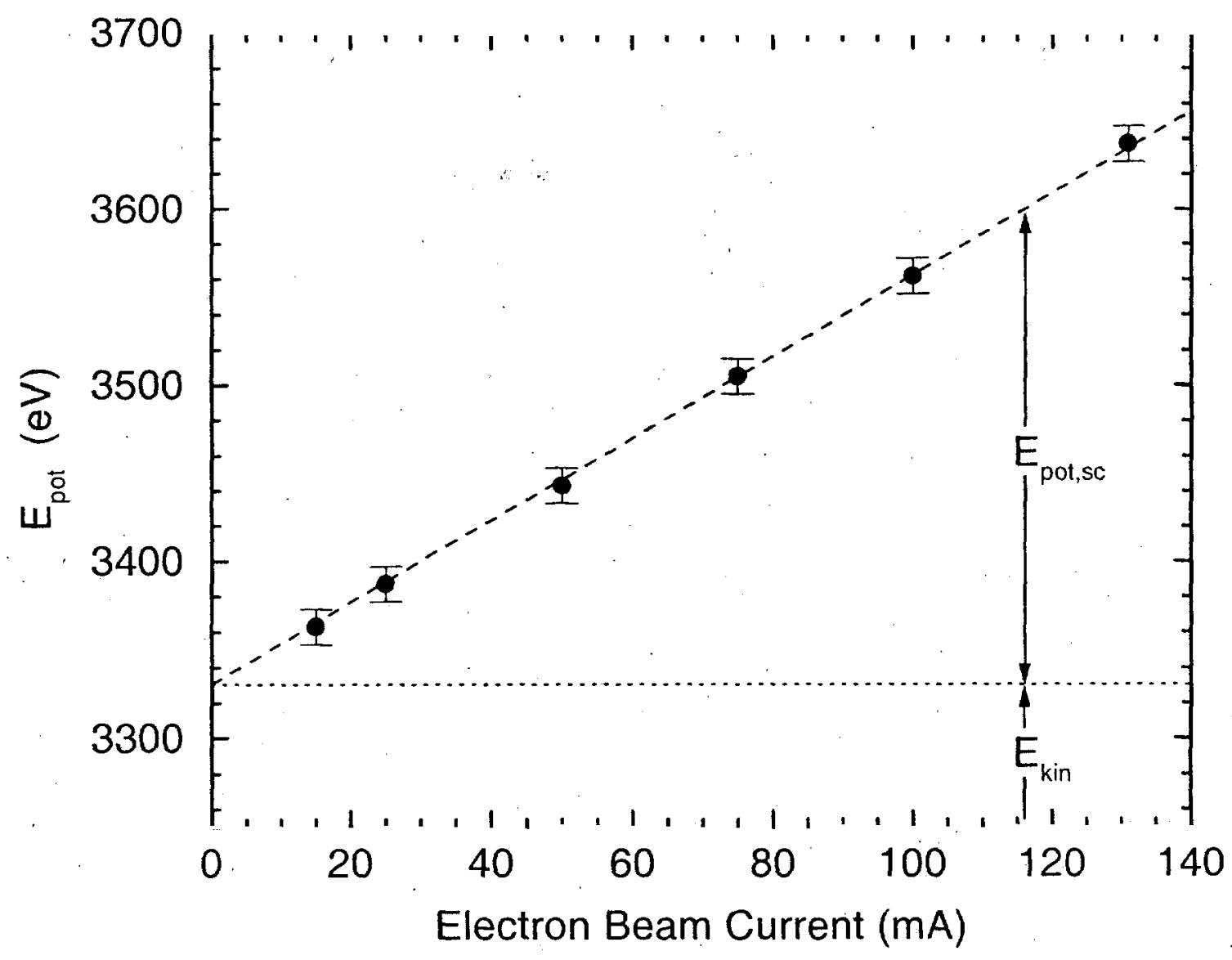

Figure 4.9: Potential difference between the electron-gun cathode and the middle drift tube, $E_{\text {pot }}$, at which the maximum emission of the dielectronic recombination KLL spectrum of lithiumlike titanium, $\mathrm{Ti}^{19+}$, was observed. Using linear regression of the acquired data points yields an space charge potential average of $2.32 \pm 0.03 \mathrm{~V}$ per $\mathrm{mA}$ of electron-beam current.

observed in this measurement is. basically, a blend of the $1 s 2 p^{2}{ }^{2} D_{5 / 2} \rightarrow 1 s^{2} 2 p^{2} D_{3 / 2}$ and $1 s 2 p^{2} D_{3 / 2} \rightarrow 1 s^{2} 2 p^{2} D_{1 / 2}$ transitions of lithiumlike titanium, $\mathrm{Ti}^{19 \div}$. Thus, the resonance occurs at an electron-beam energy of $3330.5 \mathrm{eV}$, i.e., the kinetic energy of the electron with respect to the ion. The kinetic energy of the electron, $E_{k i n}$, is equal 
to the sum of the potential energies, i.e., the energy due to the potential difference between cathode and middle drift tube, $E_{p o t}$, and the energy due to the space charge, $e U_{\text {sc }}$, (see also Figures 4.12 and 4.13 on pages 51 and 52 , respectively). Thus, the measured space charge potential is:

$$
U_{s c}=\frac{1}{e}\left(\dot{E}_{k i n}-E_{p o t}\right) \text {. }
$$

Table 4.4 gives a comparison of the measured space charge potential values and the calculated values utilizing the different models described above, i.e., the homogeneous and Gaussian electron density distributions, equations (4.20), (4.21) and (4.25). Since the measured values reflect the average space charge potential "seen" by the ions within the electron beam, the calculated values, too, represent the potential average within the volume defined by the radius $R_{c y l}$. The space charge compensation factor $f$ is inferred from the difference between the calculated and the measured values, using the relation

$$
f=1-\frac{U_{s c, e x p t}}{U_{s c, \text { theo }}}
$$

\subsubsection{Radial electron temperature}

According to Liouville's theorem the electron density in phase space ${ }^{16}$ in the vicinity of a given electron of the electron beam, i.e., in the volume $\mathrm{d} \vec{x}$ around the electron and within the momentum interval $\mathrm{d} \vec{p}$, does not change as the electron moves through electric and magnetic fields. A derivation of this theorem can be found elsewhere, e.g. $|48|$. In this reference and in several other publications (e.g. |67|) the velocity of the electrons is used instead of their momentum to define the phase space. The dimension of the phase space, however, should be equal to the dimension of the Planck constant, i.e., energy - time or in SI units: $\underset{\mathrm{S}}{\mathrm{kgm}} \mathrm{m}^{2}$. Therefore, the momentum of the particle has to be used instead of the velocity, especially, when relativistic effects become significant and, thus, the mass noticeably depends on the velocity.

\footnotetext{
${ }^{15} \mathrm{~A}$ multi-dimensional space based on the spatial coordinates and the momentum of the particle.
} 
Table 4.4: The measured, $U_{s c, e x p t}$, and calculated, $U_{s c, t h e o}$, space charge potential values are compared and the space charge compensation factor, $f$, inferred from their difference according to eq.(4.33). Measured and calculated values represent the potential average within the volume defined by the radius $R_{c y l}$ which is calculated using $R_{\text {cyl }}=\frac{1}{2} \sqrt{ } 5 \ln 5 r_{0}$, where $r_{0}$ is the effective beam radius from eq.(4.4). Following parameters have been used: $B=3 \mathrm{~T}, B_{c}=0 \mathrm{~T}, r_{c}=1.5 \mathrm{~mm}, T=1400 \mathrm{~K}$, and $U=3330.5 \mathrm{~V}$.

\begin{tabular}{cccccc}
$I$ & $U_{\text {sc }, \text { expt }}$ & \multicolumn{2}{c}{$U_{\text {sc,theo }}(\mathrm{V})$} & \multicolumn{2}{c}{$f$} \\
$\mathrm{~mA}$ & $\mathrm{~V}$ & Homogenous & Gaussian & Homogenous & Gaussian \\
10 & -23.2 & -26.2 & -26.5 & 0.113 & 0.122 \\
25 & -58.1 & -65.4 & -66.1 & 0.112 & 0.122 \\
75 & -174.2 & -195.6 & -197.6 & 0.109 & 0.119 \\
125 & -290.3 & -324.9 & -328.3 & 0.106 & 0.116
\end{tabular}.

Under the conservation of the phase-space density of the electrons spatial compression of the beam leads to an increase in the momentum of the electrons which is equivalent to an increase in electron temperature. In particular, a compression of the beam diameter by the factor $M$ reduces the spatial confinement by the factor $M^{2}$. Neglecting electron-electron interactions, the momentum space in radial direction, thus, has to increase by a factor of $M^{2}$. Covering two degrees of freedom, the radial velocity of the electrons experience a $M$-fold increase. The temperature, which is linked to the kinetic energy, i.e., to the square of the velocity of the particles, increases by a factor of $M^{2}$, like the momentum space itself. Assuming an initial electron temperature equal to the temperature of the electron-gun cathode, an estimate of the electron temperature at any given point of the beam can be derived upon knowledge of the evolution of the electron beam's spatial confinement.

In EBIT and SuperEBIT the electron beam originates from a 3-mm diameter area 
and is compressed to a diameter of $\approx 60 \mu \mathrm{m}$, and $\approx 100 \mu \mathrm{m}$, respectively, reducing the cross section of the beam $\approx 50^{2}$ times in EBIT and $\approx 30^{2}$ times in SuperEBIT. Starting with an electron temperature around 0.11 to $0.12 \mathrm{eV}$ at the cathode surface, thus, yields thermal electron energies of about $275 \mathrm{eV}$ to $300 \mathrm{eV}$ in the trap region of EBIT and of about $100 \mathrm{eV}$ to $110 \mathrm{eV}$ in the trap region of SuperEBIT. However, this estimate is based on the assumption that there are no electron-electron interactions which could transfer energy between the different degrees of freedom, especially between the radial and axial directions. Moreover, the electron mass is considered to be constant, i.e., independent of the velocity of the electrons.

\subsubsection{Velocity profile}

Several measurements of the radial distribution of the axial electron velocity in cylindrically symmetric electron beams have demonstrated that the axial velocity profile varies as a function of the radial distance $|61,60|$. The radial dependence of the axial electron velocity is due to the rigid rotation of the whole beam around the beam axis. Hence, the kinetic energy consists of a translational and a rotational part. Electrons that have more transverse kinetic energy by virtue of rotating about larger transverse circles must have smaller axial kinetic energy. Therefore, the axial velocity of the electrons decreases with increasing radial distance, i.e., the outer electrons move slower axially than inner electrons. Since Herrmann's optical theory for calculating the electron beam radius (see eq.(4.3) on page 25) does not include any radial dependence of the axial electron velocity, a quantitative evaluation of the velocity gradient is essential for a discussion of the applicability of Herrmann's theory.

The rotation of the beam is due to the presence of crossed electric and magnetic fields, i.e., $\vec{E} \times \vec{B} \neq \overrightarrow{0}$. Thus, the electrons follow trajectories where the electric and magnetic forces are balanced by the inertia of the electron. A simple diagram showing the qualitative properties of those forces is shown in Figure 4.10. Using the 

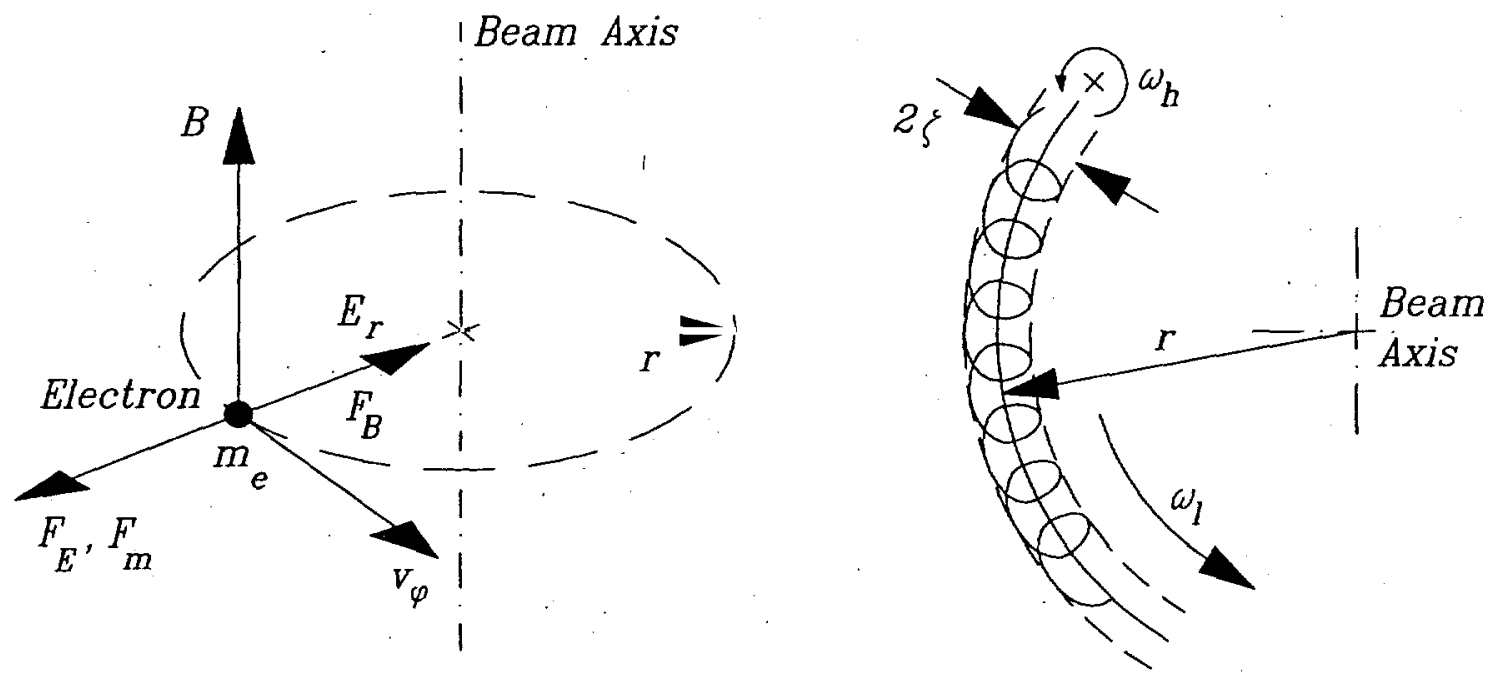

Figure 4.10: Sketch of the electron trajectory in the presence of crossed electric and magnetic fields. The geometric properties considered for the derivation of the electron trajectory include a magnetic field in axial direction and an electric field in radial direction. Figure (a) also shows the direction of the various forces on the electron, i.e., the Lorentz force $F_{B}$, the electrostatic force $F_{E}$, and the force due to the inertia of the electron $F_{m}$. The resulting electron trajectory - graphically depicted in (b) - is a superposition of a low-frequency rotation $\left(\omega_{l}\right)$ around the beam axis and a high-frequency rotation $\left(\omega_{h}\right)$ around the magnetic field lines. The quantity $\zeta$ describes the radius of this fast cyclotron motion.

notation introduced in that Figure the equation of motion is given by:

$$
\begin{aligned}
\vec{F}_{m}+\vec{F}_{E}+\vec{F}_{B} & =0 \\
\frac{m_{e}\left|\vec{v}_{\varphi}\right|^{2}}{r} \cdot \frac{\vec{r}}{r}+e \vec{E}_{r}+e \vec{v}_{\varphi} \times \vec{B} & =0 .
\end{aligned}
$$

Since the vectors $\vec{r}, \vec{E}_{r}$, and $\vec{v}_{\varphi} \times \vec{B}$ are parallel, a scalar presentation of the equation above can easily be achieved, i.e.,

$$
\frac{m_{e} v_{\varphi}^{2}}{r}+e\left|E_{r}\right|-e v_{\varphi} B=0
$$

The signs already account for the fact that the electron's charge is negative. Introducing the rotational frequency

$$
\omega_{\varphi}=\frac{v_{\varphi}}{r}
$$


and the cyclotron frequency $\quad \omega_{c}=\frac{e B}{m_{e}}$

modifies eq.(4.35) into:

$$
\omega_{\varphi}^{2}-\omega_{c} \omega_{\varphi}+\omega_{\varphi} \frac{\left|E_{r}\right|}{B r}=0,
$$

which has the solutions: $\omega_{\varphi_{h, l}}=\frac{\omega_{c}}{2}\left(1 \pm \sqrt{1-\frac{4 m_{e}\left|E_{r}\right|}{e B^{2} r}}\right)$.

The subscripts $h, l$ denote the high- and low-frequency solution. For EBIT and SuperEBIT the term under the square root is close to one and, thus, the electrons gyrate around the magnetic field lines with the high-frequency $\omega_{\varphi_{h}}$ which is almost the cyclotron frequency, and they rotate around the beam axis with the much slower frequency $\omega_{\varphi_{l}}$. A sketch of the electron trajectory is shown in Figure 4.10. The transverse velocity due to the slow rotation, $v_{l}$, equals $\omega_{\varphi_{l}} r$, where $r$ is the radial distance from the beam axis. For the fast cyclotron motion the transverse velocity is $v_{h}=\omega_{\varphi_{h}} \zeta$. The radius $\zeta$ depends on the angle, $\alpha$, between the electron trajectory and the magnetic field lines, which are assumed to be parallel. In particular,

$$
\zeta=\frac{m_{e} v}{e B} \sin \alpha=\frac{v}{\omega_{c}} \sin \alpha .
$$

The total velocity $v$ is determined by the total kinetic energy $E_{k i n}$, which, neglecting the thermal motion ${ }^{17}$ of the electrons, only depends on the potential difference between the cathode and the point of interest, i.e., the sum of acceleration potential $U$ and space charge potential $U_{s c}$,

$$
E_{k i n}(r)=E_{p o t}(r)=e\left(U+U_{s c}(r)\right)
$$

The total kinetic energy of the electron $E_{k i n}$ is split into the kinetic energy in axial direction $E_{k i n, z}$, the transverse kinetic energy due to the fast rotation around the magnetic field lines $E_{k i n, h}$, and the transverse kinetic energy due to the slow rotation

\footnotetext{
${ }^{17}$ Including thermal motion does not introduce an additional radial dependence of the kinetic energy or the velocity distribution unless the temperature of the cathode is not uniform.
} 
around the beam axis $E_{k i n, l}$. Using the equations (B.1) and (B.2) from page 195 the axial velocity can be derived from the energy balance in following way:

$$
\begin{aligned}
E_{k i n} & =E_{k i n, z}+E_{k i n, h}+E_{k i n, l} \\
\gamma_{z} & =\gamma-\gamma_{h}-\gamma_{l}+2 \\
\text { where } \gamma_{i} & =\frac{1}{\sqrt{1-\left(\frac{v_{i}}{c_{0}}\right)^{2}}}
\end{aligned}
$$

and, thus,

$$
v_{z}=c_{0} \sqrt{1-\left(\gamma-\gamma_{h}-\gamma_{l}+2\right)^{2}} .
$$

A non-relativistic treatment of the energy balance yields the much simpler expression, i.e.,

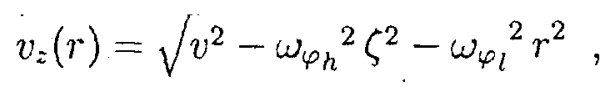

for the electron velocity in axial direction.

Determination of the axial velocity profile requires an estimate of the angle $\alpha$ between the electron trajectory and the magnetic field lines. Assuming perfect injection of the electrons, i.e., that the electrons are emitted from the cathode with trajectories parallel to the magnetic field lines, no rotation around the field lines is present and the radius $\zeta$ equals zero length. The thermal motion of the electrons, however, prevents a perfect injection. Thus, the minimum angle between the velocity vector and the magnetic flux density vector can be inferred from the ratio of the thermal to the kinetic energy of the electrons in axial directions $\left(\tan \alpha=\frac{E_{t h}}{E_{k i n, z}}\right)$. For an estimate of the electron injection angle the total energy $E_{k i n}$ was used instead of $E_{k i n, z}$. The difference in the resulting angle $\alpha$ is negligible due to the small thermal energy in comparison with the kinetic energy of the electron.

The values of the radius $\zeta$, the rotation frequencies $\omega_{c}, \omega_{h}, \omega_{l}$, and the energies $E_{k i n, h}$ and $E_{k i n, l}$ at the center and the edge of the electron beam are listed in Table 4.5. 
Table 4.5: Beam-center and beam-edge values of some quantities describing the beam rotation. In particular, these are the radius zeta of the cyclotron motion utilizing eq.(4.40), the rotation frequencies $\omega_{c}, \omega_{h}$, and $\omega_{l}$ according to eq.(4.37) and eq.(4.39), and the kinetic energies $E_{k i n, h}$ and $E_{k i n, l}$ calculated with eq.(4.36) and eq.(B.1). The parameters used for these calculations are listed in the top section of this table. $R_{c y l}$ is the radius of the beam edge assuming a homogenous electron density distribution. $E_{t h}$ the thermal energy of the electrons in the trap region (see Section 4.1.3.4). The presentation of the results was selected in order to show the impact of the various parameters upon the calculated quantities and, thus, the amount of digits used does not reflect the precision of the calculated values.

\begin{tabular}{rcccc}
\hline \multicolumn{4}{c}{ Parameters used for calculation } \\
\hline & $I$ & $U$ & $R_{\text {cyl }}$ & $E_{\text {th }}$ \\
$\mathrm{mA}$ & $\mathrm{kV}$ & $\mu \mathrm{m}$ & $\mathrm{eV}$ \\
\hline EBIT & 130 & 18 & 30.8 & 285 \\
SuperEBIT & 230 & 136 & 52.9 & 105 \\
\hline \hline
\end{tabular}

\begin{tabular}{rccccccccc} 
& \multicolumn{4}{c}{ EBIT } & \multicolumn{4}{c}{ SuperEBIT } \\
\cline { 2 - 10 } & \multicolumn{3}{c}{ Homogenous } & \multicolumn{2}{c}{ Gaussian } & \multicolumn{2}{c}{ Homogenous } & \multicolumn{2}{c}{ Gaussian } \\
& $r=0 \mu \mathrm{m}$ & $r=R_{c y l}$ & $r=0 \mu \mathrm{m}$ & $r=R_{c y l}$ & $r=0 \mu \mathrm{m}$ & $r=R_{c y l}$ & $r=0 \mu \mathrm{m}$ & $r=R_{c y l}$ \\
$\zeta(m u \mathrm{~m})$ & 2.4196 & 2.4185 & 2.4198 & 2.4185 & 0.34079 & 0.34078 & 0.34080 & 0.34078 \\
$\omega_{c}\left(10^{11} \mathrm{~s}^{-1}\right)$ & 5.0985 & 5.0984 & 5.0986 & 5.0984 & 4.1681 & 4.1680 & 4.1681 & 4.1680 \\
$\omega_{h}\left(10^{11} \mathrm{~s}^{-1}\right)$ & 5.0985 & 4.9916 & 5.0986 & 5.0057 & 4.1681 & 4.1411 & 4.1681 & 4.1447 \\
$\omega_{l}\left(10^{9} \mathrm{~s}^{-1}\right)$ & 0 & 10.7 & 0 & 9.3 & 0 & 2.7 & 0 & 2.3 \\
$E_{k i n, h}(\mathrm{eV})$ & 4.326 & 4.143 & 4.327 & 4.166 & 0.0574 & 0.0566 & 0.0574 & 0.0567 \\
$E_{k i n, h}(\mathrm{eV})$ & 0 & 0.3 & 0 & 0.2 & 0 & 0.06 & 0 & 0.04
\end{tabular}

The values in Table 4.5 clearly show, that the difference in the transverse kinetic energy between the center and the edge of the beam is at least two orders of magnitude smaller than the difference in the potential energy due to the space charge of the 
electron beam. Thus, the radial dependence of the kinetic energy in axial direction $E_{k i n, z}$ and the axial electron velocity $v_{z}$ is dominated by the space charge potential. Figure 4.11 shows the axial velocity distribution normalized to the value at the beam center, i.e., $v_{z}(r) / v_{z}(0)$. A comparison of the various types of kinetic and potential energies discussed in this section is plotted in the Figures 4.12 and 4.13.

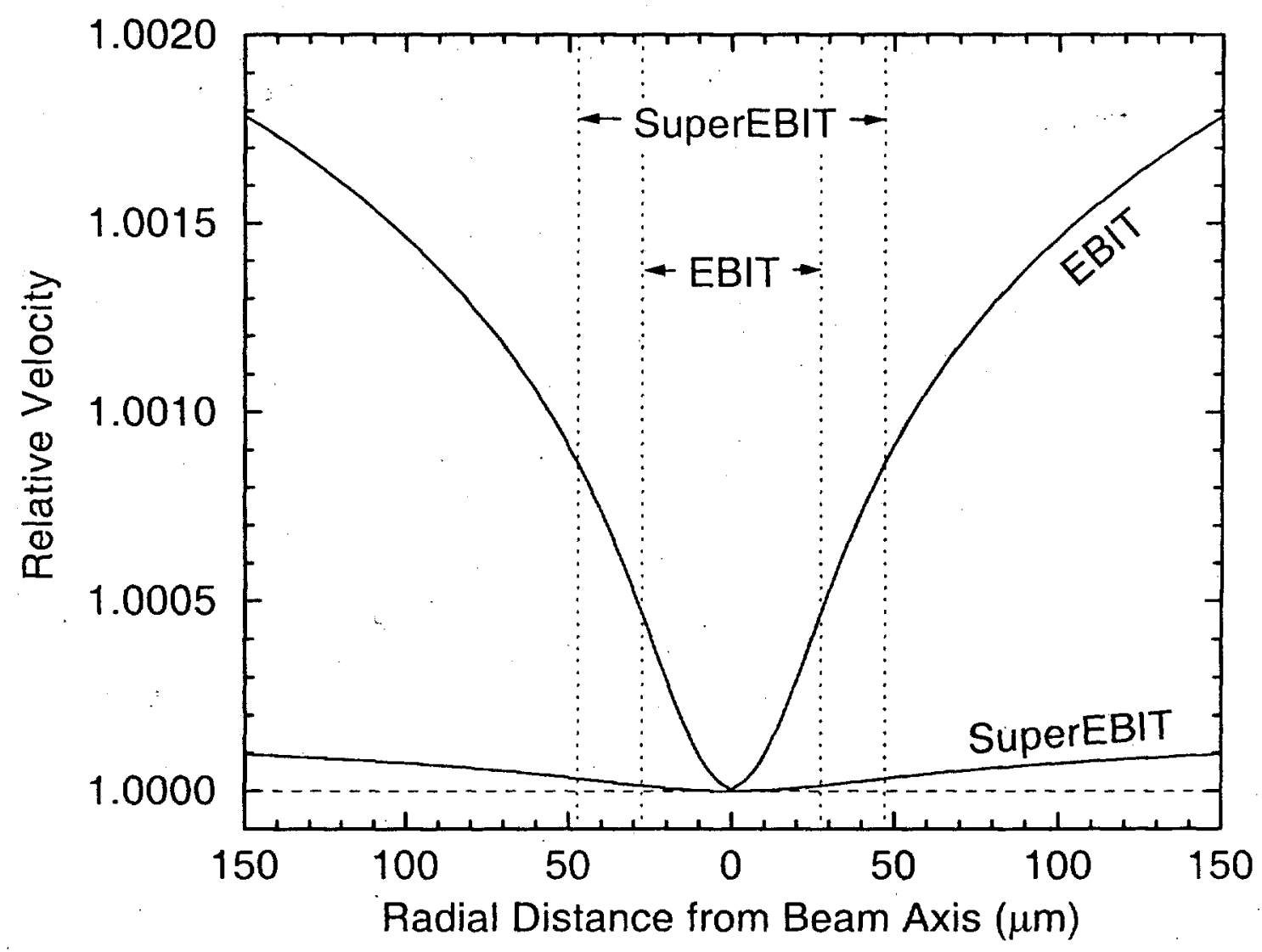

Figure 4.11: Distribution of the axial electron velocity along a cross section of the electron beam according to eq.(4.44). The values are normalized to the axial velocity at the center of the beam. The parameters used for the calculation are listed in Table 4.5, and the result of the space charge potential assuming Gaussian density distribution, eq.(4.25), was implemented into eq.(4.41). The velocity values at the beam center are: $v_{z}(0)=0.257 c_{0}$ for EBIT and $v_{z}(0)=0.613 c_{0}$ for SuperEBIT, with $c_{0}$ being the speed of light. The dotted lines represent the measured beam radii. 


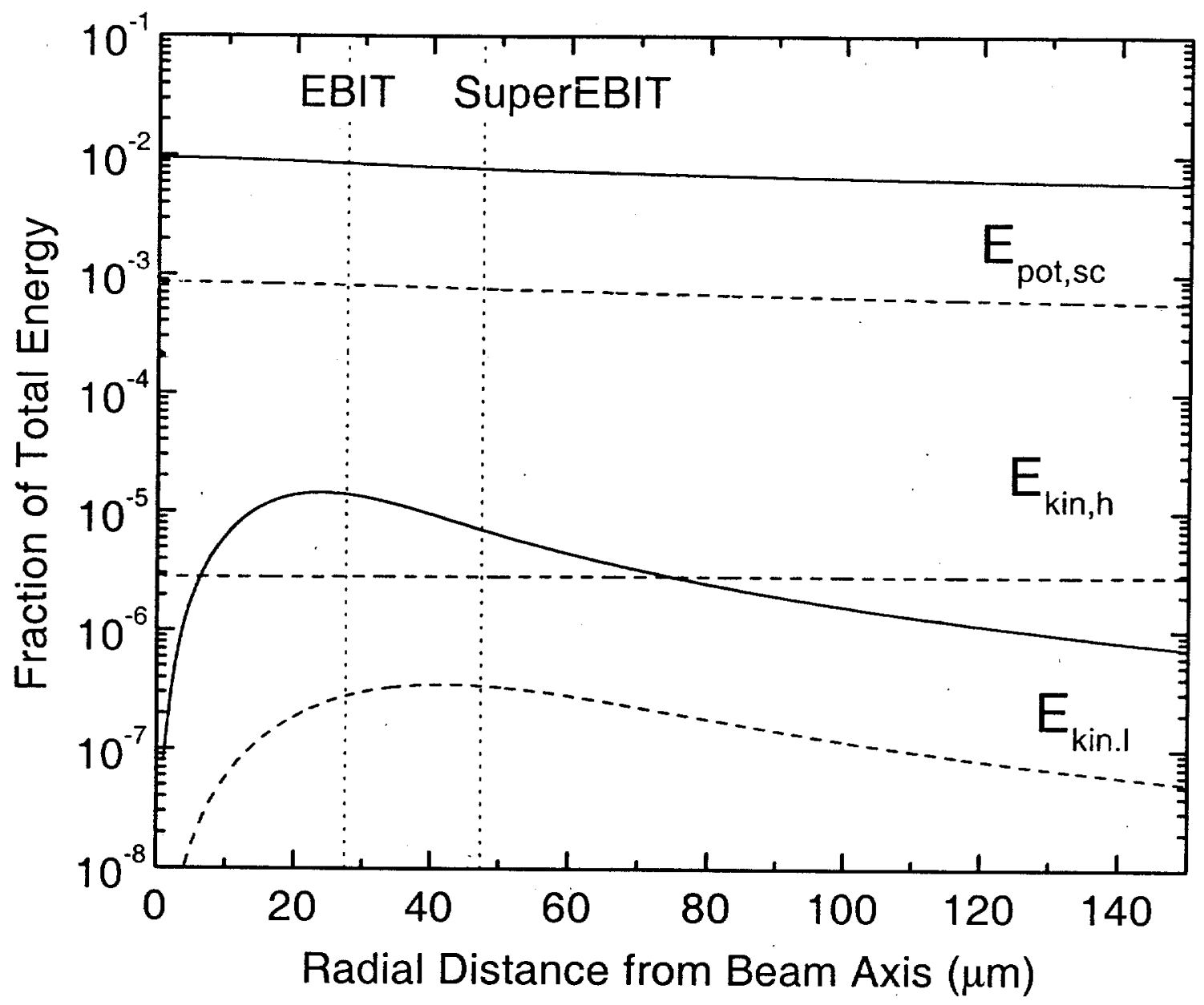

Figure 4.12: Potential and kinetic energy components of an electron-beam electron as a function of the radial distance. All energy values are given as fraction of the total energy which is defined by the potential difference between the electron-gun cathode and the middle drift tube electrode, i.e., $18 \mathrm{keV}$ for EBIT and $136 \mathrm{keV}$ for SuperEBIT. The quantities plotted are: The potential energy due to the space charge of the electron beam $E_{s c}$, the transverse kinetic energy due to the cyclotron motion $E_{k i n, h}$, and the transverse kinetic energy due to the rotation of the electron around the beam axis $E_{k i n, l}$. The solid curves are the results using the EBIT parameters, the dashed curves show the results using the SuperEBIT parameters (see Table 4.5). The dotted lines represent the measured beam radii. 

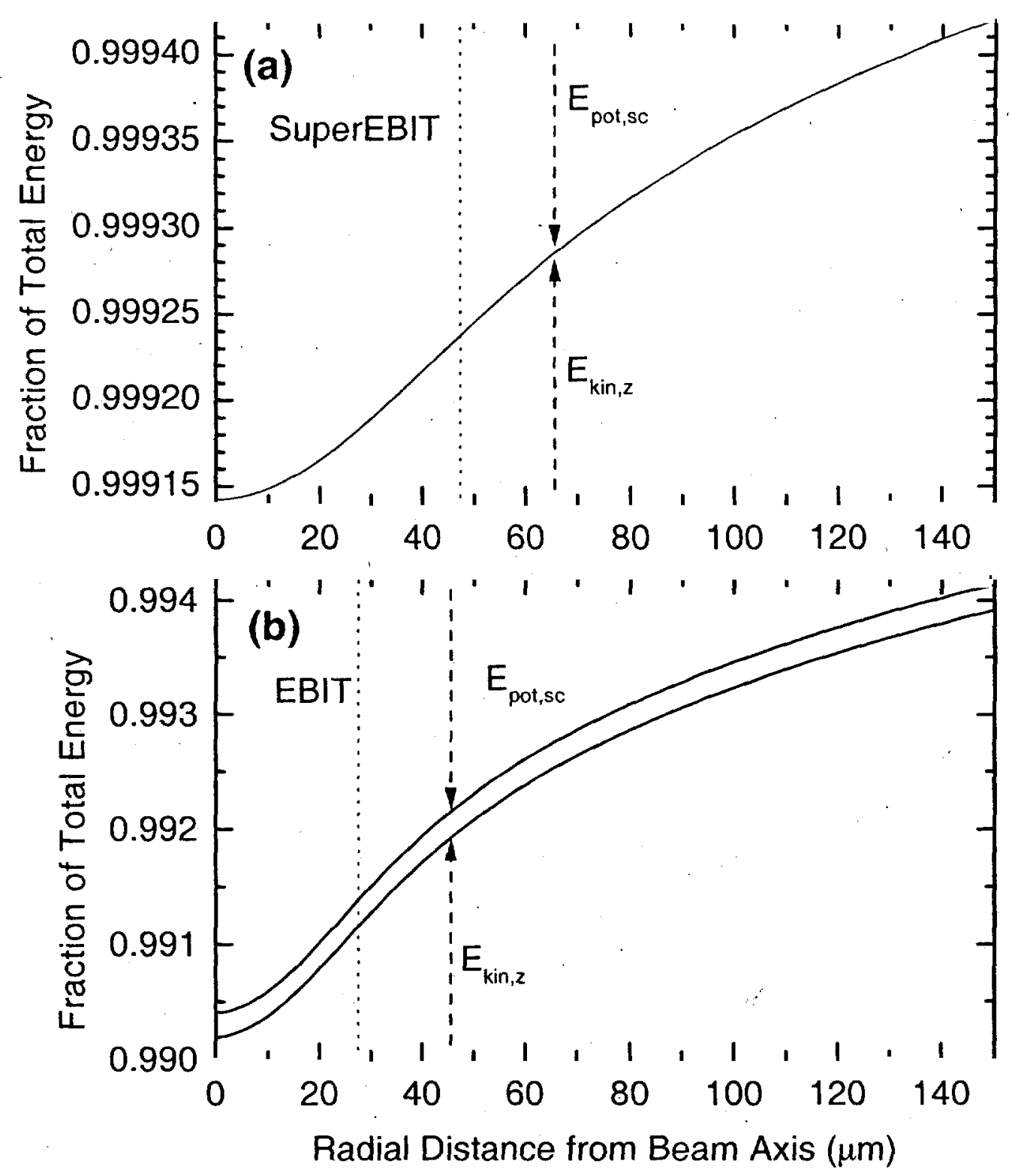

Figure 4.13: Kinetic energy components of an electron-beam electron as a function of the radial distance. All energy values are given as fraction of the total energy which is defined by the potential difference between the electron-gun cathode and the middle drift tube electrode, i.e., $18 \mathrm{keV}$ for EBIT and $136 \mathrm{keV}$ for SuperEBIT. The quantities plotted are: The total kinetic energy $E_{k i n}$ and the kinetic energy in axial direction $E_{k i n, z}$. Figure (a) presents the results for SuperEBIT, figure (b) shows the results for EBIT. The parameters used for these calculations are listed in Table 4.5). The dotted lines represent the measured beam radii. The difference between $E_{k i n}$ and $E_{k i n, z}$ is the kinetic energy in radial direction. 


\subsection{Injection systems}

There are three basic injection methods applied at the Livermore EBIT facility. In particular, these are: ballistic injection of neutral particles via a gas injection system, electrostatic injection of lowly charged ions which are produced in a metal vapor vacuum arc, and a method based on sputtering atoms and ions from a coated wire which is placed close to the electron beam at the trap region, i.e., the middle drift tube.

\subsubsection{Gas injector system}

The gas injection system allows the ballistic injection of neutral particles which are in a gas phase. The variety of injected materials includes noble gas elements ( $\mathrm{Ne}$, $\mathrm{Ar}, \mathrm{Kr}, \mathrm{Xe})$, molecules $\left(\mathrm{N}_{2}, \mathrm{CO}_{2}, \mathrm{SF}_{6}\right.$, etc.), evaporated metals ( $\mathrm{K}, \mathrm{Ti}$, Cs, etc.), and complex organic compounds which are either available in a gas phase or have a high vapor pressure at room temperature, e.g. iron pentacarbonyl $\left(\mathrm{Fe} /\left.\mathrm{CO}\right|_{5}\right)$ which has a vapor pressure of 40 Torr at $30^{\circ} \mathrm{C}$.

The EBIT facility is equipped with a continuous and a pulsed gas injection system. Both systems have differentially pumped two-chamber gas reservoirs (see Figure 4.1, page 18) and employ fine adjustable valves, i.e., a thermally controlled valve for continuous gas flow, and a current-pulse controlled valve for pulsed gas flow. For the continuous gas flow active regulation is applied to the thermo valve which allows to maintain a constant pressure in the reservoir regardless of the pressure on the high-pressure end, i.e., the gas feeding line. The feeding line is connected to the gas cylinder of choice, usually equipped with a regulator. Keeping the pressure in the feeding line just a little bit above one atmosphere prevents possible contamination with air.

The design of the differential pumping scheme allows to change the pressure in the second stage of the gas reservoir (gas injector pressure) from the $1 \cdot 10^{-6}$ Torr $\left(1.33 \cdot 10^{-4} \mathrm{~Pa}\right)$ level to the $1 \cdot 10^{-9}$ Torr $\left(1.33 \cdot 10^{-7} \mathrm{~Pa}\right)$ level within a few minutes. 
Thus, depending on the position of the thermo valve any injection pressure within that interval can be selected conveniently. One disadvantage of using a thermo valve is that in case of a power failure no heating current can be applied and the valve opens.

Although the gas injector pressure is only in the $10^{-9} \ldots 10^{-7}$ Torr range, it is still several orders of magnitude higher than the pressure in the drift tube sections, where it drops to the $10^{-11}$ Torr level and below. The exact pressure in the trap region is unknown since it is below the sensitivity of the pressure gauges, in particular, ion gauges, employed which is the $10^{-11}$ Torr $\left(1.33 \cdot 10^{-9} \mathrm{~Pa}\right)$ level. Besides, the pressure gauges are mounted too far away from the trap area to allow an accurate measurement of the true rest gas pressure in the trap region itself.

Since the pressure in the trap region is unknown, the absolute value of injected and trapped particles can only be estimated. Considering a gas with a Maxwellian velocity distribution the product of the free mean path $\Lambda$ and the gas pressure $p$ is

$$
\Lambda \cdot p=\begin{aligned}
& k T \\
& \sqrt{2} \sigma
\end{aligned},
$$

where $k$ is the Boltzmann constant, $T$ the absolute temperature, and $\sigma$ the cross section for atom-atom (or molecule-molecule) collisions. At a given temperature the product $\Lambda \cdot p$ is constant, e.g. for air at room temperature it is:

$$
\Lambda \cdot p \approx 10^{-4} \text { Torr } \cdot \mathrm{m} \approx 1.33 \cdot 10^{-2} \mathrm{~Pa} \cdot \mathrm{m},
$$

At a pressure of $10^{-6}$ Torr the free mean path of the gas particles equals $10^{2} \mathrm{~m}$. The distance between the apertures in the differentially pumped gas reservoir is, however. only in the $10^{-1}-\mathrm{m}$ range. Thus, there are basically no collisions between the gas particles along their way through the gas injector and the injected gas particles follow ballistic trajectories, i.e., trajectories which are solely determined by the initial momentum of the particle. Only particles within a certain phase-space volume manage to exit through the outlet aperture, i.e., the orifice between the injection system and the EBIT vacuum chamber. Gas particles which are outside this specific phase-space 
volume strike the walls of the injection chambers and most of these particles are removed by the vacuum pumps. If not carried away by the pump some of the particles might actually escape through the exit aperture after some reflections on the chamber walls. These "stray" particles, however, are unlikely to cross the electron beam and, thus, only increase the gas load onto the EBIT vacuum system.

Considering that for nitrogen molecules at room temperature the Maxwellian velocity distribution has its maximum at about $420 \frac{\mathrm{m}}{\mathrm{s}}$, the effect of gravitation on the trajectories of the injected particles is in the $\mu \mathrm{m}$ regime. Thus, the amount of injected particles that actually cross the electron beam was estimated by applying the rules of geometrical optics. A sketch of the geometric properties of the injection system is shown in Figure 4.14. Using the notation introduced in this figure the solid angle of the injected gas particles that intersect with the electron beam is given by

$$
\begin{aligned}
& \qquad \Omega_{b}=\psi \sin \varphi \\
& \text { where } \quad \psi=2 \arctan \frac{d_{b}}{2 x_{b}} \\
& \text { and } \quad \varphi=2 \arctan \left[\min \left(\frac{h_{i}}{2 x_{i}}\right)\right] .
\end{aligned}
$$

Thus, of all particles injected into the $2 \pi$ half sphere, only the fraction $\frac{\Omega_{b}}{2 \pi}$ actually crosses the electron beam. For EBIT this fraction, which represents the efficiency of the injection method, is about $3 \cdot 10^{-7}$. The density of the injected gas at the intersection with the electron beam is indirect proportional to the expansion of this "gas jet" which is equal to the ratio of the surface of the half sphere $A_{2 \pi}$ which originates at the center of the injector nozzle to the cross section of this nozzle $A_{0}$, i.e.,

$$
c_{e x p}=\begin{gathered}
A_{2 \pi} \\
A_{0}
\end{gathered}=\frac{8 x_{b}{ }^{2}}{h_{0}{ }^{2}} .
$$

The reciprocal values of the expansion coefficient $c_{\exp }$ is in the order of $10^{-6}$ for the EBIT and SuperEBIT gas injection systems and, thus, the density of the neutral gas beam is reduced by about 6 orders of magnitude on its way from the injector nozzle 


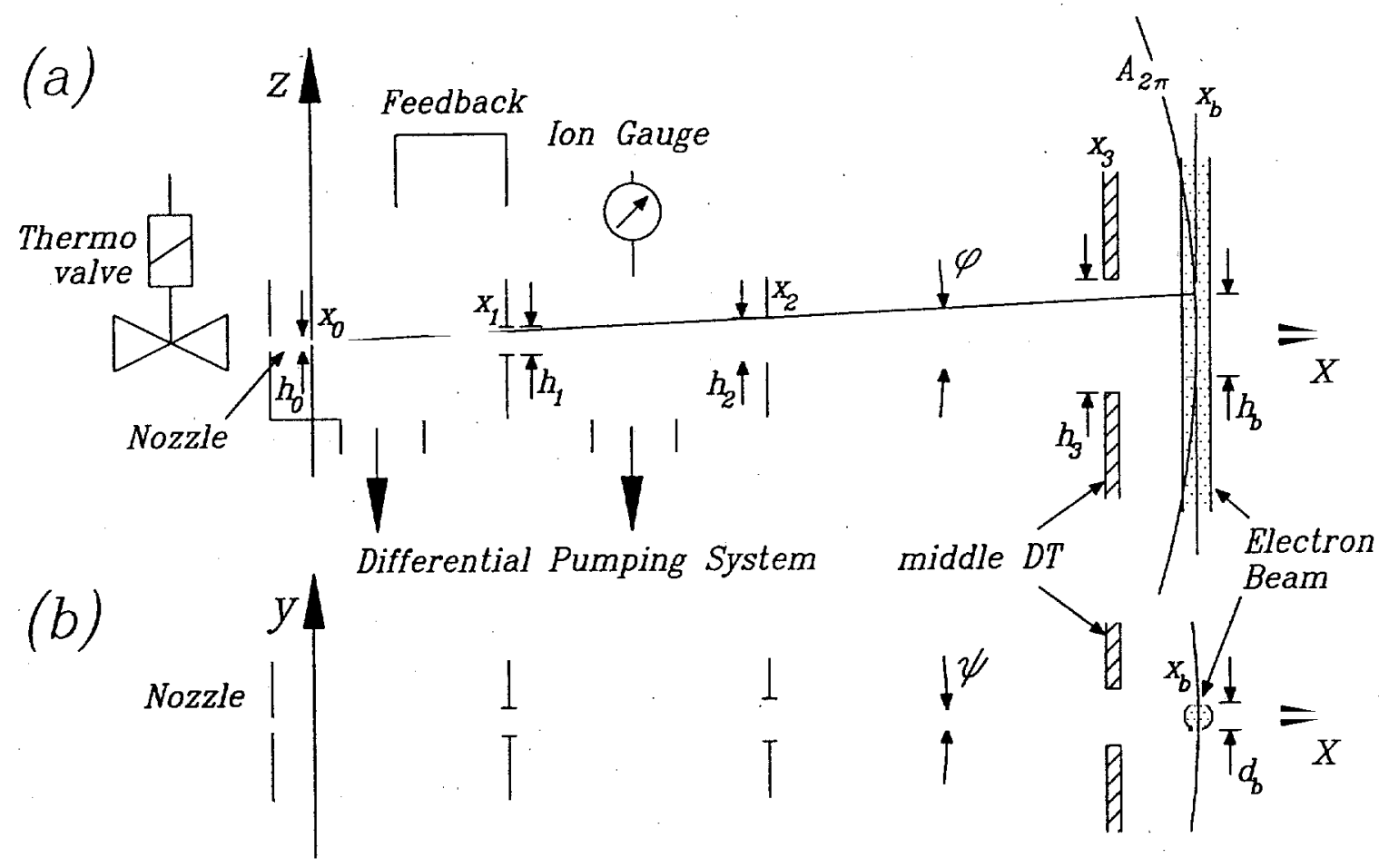

Figure 4.14: Schematic layout of the gas injection system, (a) side view, top view (b). The injected beam is collimated by a set of small orifices with the diameter $h_{i}$ along the line of sight from the injector nozzle to the electron beam. The opening polar angle $\varphi$ defines the quantity $h_{b}$, i.e., the height of the electron beam which is exposed to the "gas jet". In the azimuthal plane the opening angle $\psi$ is determined by the diameter of the electron beam $d_{b}$.

to the electron beam. The parameters used for calculating the injection efficiency and expansion coefficient are listed in Table 4.6.

For an ideal gas the density $n$ depends only on the pressure $p$ and the temperature $T$ of the gas, in particular.

$$
n=\begin{gathered}
p \\
k T
\end{gathered}
$$

$k$ being the Boltzmann constant. Determination of the "gas jet" density at the intersection with the electron beam requires knowledge about the pressure at the injector nozzle, i.e., the pressure in the first gas reservoir. Although, the ion gauge which is used for actively regulating the thermo valve only monitors the pressure 
Table 4.6: Estimated values for the solid angle $\Omega_{b}$ of the ballistically injected particles, the efficiency of the injection method $c_{e f f}$, i.e., the fraction of the injected particles which intersect with the electron beam, and the reciprocal values of the beam expansion $\frac{1}{c_{\text {exp }}}$. The assignment of the geometric quantities can be taken from Figure 4.14.

\begin{tabular}{cccc}
\hline index & $x_{i}$ & $h_{i}$ & $\varphi_{i}$ \\
& $\mathrm{~mm}$ & $\mathrm{~mm}$ & ${ }^{\circ}$ \\
\hline 0 & 0 & 0.75 & 90.0 \\
1 & 150 & 1.59 & 1.21 \\
2 & 300 & 2.38 & 0.91 \\
3 & 515 & 6.35 & 1.41 \\
$\mathrm{~b}$ & 520 & 4.07 & 0.91 \\
\hline
\end{tabular}

\begin{tabular}{rccccc}
\hline \hline & $d_{b}$ & $\psi_{i}$ & $\Omega_{b}$ & $c_{\text {eff }}$ & $\frac{1}{c_{\text {exp }}}$ \\
& $\mu \mathrm{m}$ & $\circ$ & $\mathrm{sr}$ & & \\
EBIT & 61.6 & 0.0068 & $1.9 \cdot 10^{-6}$ & $3.0 \cdot 10^{-7}$ & $1.0 \cdot 10^{-6}$ \\
SuperEBIT & 105.8 & 0.0117 & $3.2 \cdot 10^{-6}$ & $5.1 \cdot 10^{-7}$ & $1.0 \cdot 10^{-6}$ \\
\hline \hline
\end{tabular}

in the second reservoir, the pressure in the first chamber can be inferred from that measured value, because these two quantities are linked together by the geometric properties of the differential pumping scheme including the pumping power employed. For the EBIT gas injection system the pressure in the first chamber is about 80 times higher than the pressure measured in the second chamber [57]. Thus, assuming a gas injector pressure of $10^{-8}$ Torr, i.e., a density of about $3.3 \cdot 10^{8} \mathrm{~cm}^{-3}$ at $T=293 \mathrm{~K}$, would yield a neutral density of $2.8 \cdot 10^{4} \mathrm{~cm}^{-3}$ at the location of the electron beam. However, the pressure at the location of the nozzle of the gas injector is much higher 
than in the second chamber, and this estimate, thus, represents a lower limit of the neutral density at the trap region.

As mentioned above, the Livermore EBIT facility is also equipped with a pulsed gas.injection system which has a pulsed supersonic beam valve ${ }^{18}$. The amount of gas released into the trap region depends on the pressure gradient and the pulse duration, which is in the order of $10^{5} \mathrm{~Pa}$ and 30 to $60 \mu \mathrm{s}$, respectively. Typically, the pressure in the gas injector is about $16 \mathrm{psi}\left(1.110^{5} \mathrm{~Pa}\right)$ at the high-pressure end of the pulsed supersonic valve and in the mid $10^{-9}-$ Torr $\left(1.33^{-11} \mathrm{~Pa}\right)$ range at the low-pressure end. The pulsed injection technique allows to inject a higher amount of particles without increasing the overall gas load within one duty cycle. Moreover, when using the continuous injection method the constant stream of neutrals not only degrades the ultra high vacuum in the trap region but also limits the achievable charge balance due to charge exchange between highly charged ions and neutrals of the same species.

\subsubsection{Metal Vapor Vacuum Arc (MeVVA)}

Another pulsed method for injection of elements which is available at the Livermore EBIT facility is a Metal Vapor Vacuum Arc (MeVVA). This miniature arc has been developed at the Lawrence Berkeley National Laboratory by Brown et al. $\{69,70 \mid$ as an ion source for all metallic elements.

A high-current electrical discharge between anode and cathode electrode forms several arc streams and, thus, hot spots at the location of the cathode. Through these hot spots material from the cathode enter the arc plasma: The space charge of the electrons in the arc streams is sufficient to pull the positively charged ions towards the anode. When the ions get close enough to the anode they see the strong extraction potential applied between the anode electrode and the so called focus electrode. At an focus-anode potential difference of $8 \mathrm{kV}$ the extracted ion beam current of the miniature are is about $5 \mathrm{~mA}|70|$. The pulse duration is in the order of

\footnotetext{
${ }^{18}$ Manufactured by R.M. Jordan Co. (see also [68]).
} 
$20 \mu$ s and, thus, about $10^{-7} \mathrm{C}$, i.e., $6.24 \cdot 10^{11}$ elementary charges, are extracted per pulse. Doubly charged ions are the most common species in the extracted ion beam of the miniature arc $|71|$, and thus, each MeVVA pulse yields about $3 \cdot 10^{11}$ ions.

The ions which are extracted through holes in the anode and focus electrode move towards the EBIT collector. An Einzel lens, which is mounted between the focus electrode of the MeVVA and the electron beam collector, is used to project the extracted ions onto the beam axis past the collector region. Despite implementation of the electrostatic Einzel lens only about $0.2 \%$ of the ions extracted from the MeVVA are successfully injected into the electron beam. As soon as the ions hit the electron beam they are radially confined by the electron beam's space charge.

To ensure that confinement in axial direction takes place at the middle drift tube, the potential of the drift tubes is set approximately equal to the anode potential of the MeVVA which is basically the starting potential of the ions. Additionally, the potential of the top drift tube is moved below the potential of the middle drift tube during the injection process. When the decelerated ions reach the middle drift tube region the potential of the top drift tube is raised again reestablishing the potential well in axial direction. The potential distribution along the beam axis during injection of the positively charge MeVVA ions and during ionization of the trapped ions is sketched in Figure 4.15.

By changing the electric connections for cathode and trigger electrodes the arc discharge burns between anode and trigger wire and, thus, the MeVVA plasma contains the trigger wire material which can be injected into EBIT. In fact, utilizing a trigger pulse to initiate the discharge always causes sputtering of a few particles from the trigger electrode material. Thus, the atomic number of the trigger wire element should always be smaller than the atomic number of the cathode element, when two different elements for the trigger wire and the cathode are selected ${ }^{19}$. Additionally,

\footnotetext{
${ }^{19} \mathrm{At}$ a given electron beam energy the charge state of an ion is proportional to its atomic number and, therefore, heavier elements experience a stronger trapping force than lighter elements.
} 


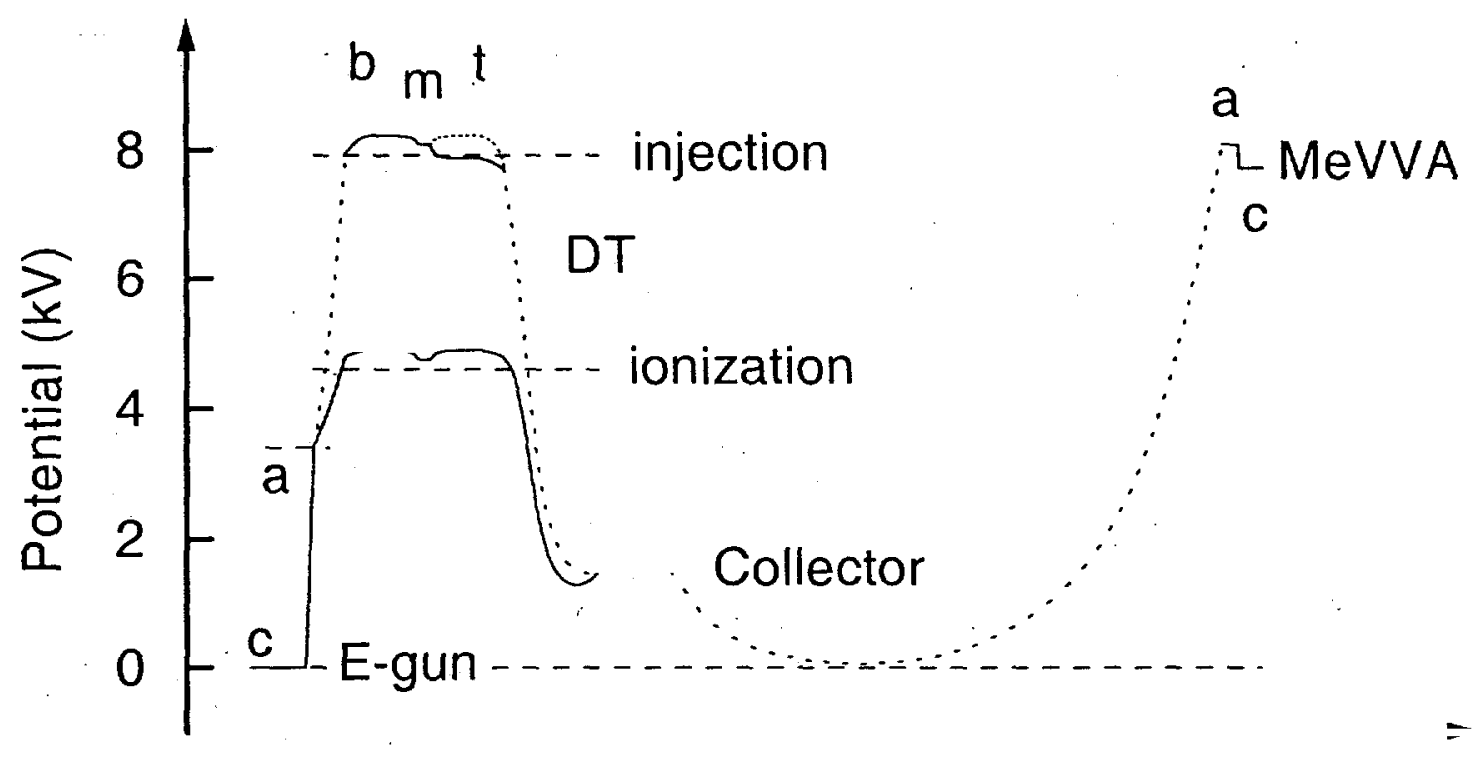

\section{Distance along beam axis}

Figure 4.15: Qualitative shape of the potential along the beam axis from the electron gun (E-gun) to the MeVVA - a... anode, c...cathode. During injection of the MeVVA ions the potential of the middle $(\mathrm{m})$ drift tube is equal to the potential of the MeVVA anode and higher than the top ( $t$ ) drift tube potential. When the ions reach the middle drift tube the top drift tube potential is raised and the ions are trapped. The dashed lines are the floating potential for the whole drift tube assembly (DT) and the E-gun assembly which includes the collector. In EBIT the E-gun cathode sits at ground potential. The SuperEBIT facility allows to bias the E-gun assembly to almost $-200 \mathrm{kV}$.

the neutrals in the plasma discharge condensate when they hit the surrounding walls which can yield a thin coating of cathode material onto the trigger wire. This thin layer of cathode material on the trigger wire enables to inject both elements simultaneously when switching cathode and trigger electrodes as described above.

The MeVVA injection method is, however, limited to conducting materials only. Successful implementation of elements also depends on the ability to machine the material into the required shape. Furthermore, a certain heat resistivity is important, i.e., the melting point should be high enough that the arc does not cause a melt down of the MeVVA material. 


\subsubsection{Wire probe}

This injection method which is based on ion sputtering has been developed recently [72| and requires only very small quantities (a few nanograms) of source material permitting the use of rare and/or expensive materials. The material of choice is coated onto a wire which is placed close to the electron beam in the middle drift tube region. Typically, the tip of the wire is about $1 \mathrm{~mm}$ away from the beam axis. The wire has to be biased in order to not significantly affect the potential distribution in the trap. Since the ion densities at a given radius depend exponentially on the ratio of potential energy to kinetic energy (Boltzmann distribution), it is most likely that the ablation of the material is due to sputtering by lowly charged ions.

\subsection{Vacuum and Cryogenics}

The ionization rate and excitation rate of the highly charged trapped ions due to the electron beam strongly depends on the collisional rate and, thus, on the electron density of the beam. Radial compression of the beam is, therefore, essential and the strong, stationary magnetic field required for this task is only achievable utilizing superconducting electromagnets. The critical temperature of the superconducting material employed at EBIT and SuperEBIT $\left(\mathrm{Nb}_{0.6} \mathrm{Ti}_{0.4}\right)$ is $T_{c}=9.8 \mathrm{~K}$. To cool the magnet below the critical temperature the Helmholtz coils are submerged in a liquid helium bath. The cryogenic design of EBIT includes also a liquid nitrogen shield around the liquid helium reservoir to reduce the thermal load onto the liquid helium reservoir from the surrounding room-temperature environment. Liquid nitrogen is also used to cool the collector, which due to the impact of the electrons carries a heat load of up to $400 \mathrm{~W}$.

The liquid nitrogen shield is being filled up automatically every hour, the collector cooling circuit is set to a permanent flow during operation. A 6000-l tank which supplies the liquid nitrogen for both machines, EBIT and SuperEBIT, has to be filled every four days. The liquid nitrogen is, however, also used for all cryogenically cooled 
detectors and for nitrogen gas supply and, hence, the liquid nitrogen consumption does not reflect solely the needs of the EBIT devices. During continuous operation, i.e., 24 hours a day, seven days a week, the consumption of liquid helium is about $4 \mathrm{l}$ per hour for EBIT and $10 \mathrm{l}$ per hour for SuperEBIT. The liquid helium is supplied in form of 250-1 or 500-1 dewars. The superconducting coils stay always connected to the power supply through the magnet leeds which unfortunately are also very good thermal conductors. Currently, no system is installed to recycle the boiled-off helium. However, the helium gas is reused to create a helium atmosphere for $\mathrm{x}$-ray spectrometers targeting the energy range between $3 \mathrm{keV}$ and $20 \mathrm{keV}$.

EBIT requires a very high vacuum at the location of the trap in order to avoid quenching of the high charge states of the trapped ions due to charge exchange with the rest gas. The desired pressure of $10^{-11}$ Torr and below is achieved by several ion pumps and cryopumps. Additionally, the liquid nitrogen shield and the liquid helium. reservoir work as big cryotraps where almost all rest gas particles condensate. Thus, most of the rest gas present in the trap region comes from outside the cryogenically cooled regions, especially from the electron gun and from the MeVVA. A decrease of the pressure in these two regions reduces the gas load in the trap area, narrows the focus diameter, improves the stability of the electron beam, and increases the amount of ions injected from the MeVVA device. All these effects contribute to a higher density of highly charged ions in the trap. During operation the pressure in the electron gun and in the upper chamber, i.e., between the drift tubes and the MeVVA, should be in the $10^{-9}$ Torr range.

There are several interlocks installed that are triggered by the pressure in the EBIT vacuum vessel. In particular, the observation ports are equipped with pneumatically controlled valves which shut if the pressure in EBIT exceeds the $1 \cdot 10^{-6}$-Torr limit. The valve that separates the gas injector from EBIT is additionally interlocked with the pressure in the gas injector reservoir, and it is being closed when that pressure exceeds the $1 \cdot 10^{-6}-$ Torr limit. The electron-gun filament power supply, which provides 
the heating current for the cathode, is interlocked with the electron-gun pressure. The power supply is turned off above an electron-gun pressure of $1 \cdot 10^{-7}$ Torr to avoid destructive oxidation of the cathode.

\subsection{Operation}

Since the energy of the quasi monoenergetic electron beam is mainly determined by the potential difference between the electron-gun cathode and the middle drift tube, it can be controlled with only one power supply. In particular, this is the power supply of the whole drift tube assembly (DT-level in Figure 4.15) which amplifies an analog low-voltage input signal. This input signal can be steady state or of any time-dependent form ${ }^{20}$ limited, of course, by the slew rate of the power supply ${ }^{21}$. The DT potential is monitored using a high-precision high-voltage divider ${ }^{22}$. Due to the limited rise time of the power supply and the impedance of the drift tube assembly the shape of the DT potential can be slightly different from the analog input signal.

The potential of each drift tube, i.e., bottom, middle, and top drift tube, is adjusted by additional power supplies, which create potential differences on top of the overall DT potential. Thus, these power supplies and all the signal-processing instruments necessary for controlling the drift tubes have to float on the DT potential. The 110$\mathrm{V}, 60-\mathrm{Hz} \mathrm{AC}$ voltage needed for the instruments is created by a generator which is connected to a motor through an electrically insulating axle and, thus, also sits on the floating DT potential. Optical fiber links allow electric insulation of all input/output signals between the main EBIT control racks and the control units which float on the DT potential.

The signal processing logistic includes a time module with 32 synchronized 3.5-

\footnotetext{
${ }^{20} \mathrm{~A}$ high-precision function generator - Hewlett Packard. HP $3314 \mathrm{~A}$ - is used to produce the time-dependent low-voltage input signal.

${ }^{21}$ At EBIT a Trek 20/20 power supply is used which has a slew rate of $350 \frac{\mathrm{V}}{\mu \mathrm{s}}$.

${ }^{22}$ Commonly referred to as "Ross probe" - manufactured by Ross Engineering Company, CA.
} 
$\mathrm{V}$ outputs with a minimum width of $0.1,1.0$, or $10 \mathrm{~ms}$. Through a graphical user interface, any sequential pattern of gates can be set for each of the 32 channels. Optional loops of variable length and repetition increase the flexibility of the graphical interface and allow implementation of any kind of periodical timing pattern with duty cycles from the ms-range up to several minutes or even hours if necessary. The timing cycle can also be stopped or restarted at any time utilizing different interrupt options. The restart (or abort) feature is, for example, useful in case the injection of ions from the MeVVA has failed or the electron beam crashed, which, of course, empties the trap and, thus, instead of wasting a whole duty cycle a new injection can be started after activating the restart option. For measurements on very high-Z elements, however, it can be advantageous to increase the trapping time after successful injection occured and, thus, the stopping option comes in handy.

A typical timing cycle includes the injection of the element of choice, the ionization until the high charge states are obtained, the period of data acquisition, and dumping of the ions before high $\mathrm{Z}$ elements intrinsic to the EBIT and SuperEBIT device accumulate in the trap. Those "intrinsic" elements are, for example, barium and tungsten from the electron gun assembly which eventually reach the trap and displace lower-Z elements. The fact that heavier elements reach a higher charge state at the same electron beam energy enables to cool ions by admixing lower- $\mathrm{Z}$ elements. In thermal equilibrium, i.e., at the same kinetic energy, the lower- $Z$ elements are more likely to leave the trap since the potential energy is proportional to the charge of the ion. This method of cooling is referred to as evaporative cooling and is discussed in detail elsewhere $|45,73|$.

\subsection{EBIT versus SuperEBIT}

Historically, the development of SuperEBIT was an upgrade of EBIT with the main goal to increase the upper limit of the applicable electron beam energy. The drift tubes, optimized for trapping, exciting, and ionizing the highly charged ions, how- 
ever, are designed to withstand maximal $35 \mathrm{kV}$ of potential difference between the drift tube electrodes and the surrounding EBIT vacuum vessel. In particular, EBIT can currently be operated at a maximum drift tube potential of only $15 \mathrm{kV}$ to $20 \mathrm{kV}$, at SuperEBIT a drift tube bias of $28 \mathrm{kV}$ was successfully applied in a recent experiment. Further increase of the electron beam energy is achieved by negatively biasing the electron gun. A new collector had to be designed which keeps the potential difference to the electron-gun cathode constant, in particular, to about $2 \mathrm{kV}$. Therefore, at the collector the electrons have only a fraction of the energy they had at the middle drift tube and the heat load onto the collector and the hardness of the Bremsstrahlung are tremendously decreased. So far, the lowest potential with which electrons have been launched was $-207 \mathrm{kV}$ - during an experiment performed by Crespo et al. [33]. Additional steering elements for the electron beam are required since the SuperEBIT collector sits about $125 \mathrm{~cm}$ above the location of the "old" or "original EBIT" collector, which was kept in the machine.

The cooling system for all electrodes of the electron gun and collector assembly has to float at the electron-gun potential, too. Thus, the automatized liquid nitrogen cooling circuit can not be utilized for cooling the SuperEBIT collector. A separate cooling circuit is installed with kerosene as coolant ${ }^{23}$. This kerosene cooling system is currently the weakest link in the chain of subsystems which are essential for SuperEBIT operation.

\subsection{Other EBIT facilities}

Cloning in the world of physics is less controversial than in the biological or medical fields, in fact, it is certainly a sign of success if experimental devices are duplicated.

\footnotetext{
${ }^{23}$ Freon, which would be a much better coolant in terms of heat capacity and electric insulation is, unfortunately, a potential environmental hazard, especially in a high-voltage environment, where the presence of an electric spark could cause chemical reactions creating highly toxic byproducts of the carbon-hydrate fluorides.
} 
In case of EBIT several devices - copies and standalone developments - exist to date worldwide:

- Clarendon Laboratory, Department of Physics, University of Oxford, Oxford UK $|74|$.

- Atomic Physics Division, National Institute of Standards and Technology, Gaithersburg, MD, USA $|75|$.

- University of Electro-Communications, Chofu, Tokyo, Japan |76|.

- Max Planck Institut für Plasmaphysik, Bereich Plasmadiagnostik, EURATOM Association, Berlin, Germany |77|.

- Supposedly, there are also two EBIT devices in Moscow but they are not operational due to the lack of financial support.

The existence of all these EBIT facilities demonstrate the feasibility of this device for production and investigation of highly charged ions. Moreover, it clearly shows the increasing interest of the physics community of various fields, e.g., atomic physics, plasma physics, astrophysics, and surface science, in highly charged ions. 


\section{X-ray Spectrometers}

Two different groups of $\mathrm{x}$-ray spectrometers are currently used at the Livermore EBIT facility, i.e., low-resolution solid-state detectors and high-resolution crystal spectrometers. The solid-state detectors are the work horses in respect to broad-band surveys of the $x$-ray radiation emitted from the ion trap region. A short description of solid-state detectors, in particular, high-purity germanium detectors, is given in Section 5.1 below. Among the high-resolution crystal spectrometers used at EBIT and SuperEBIT the following Sections focus on the von Hámos and the DuMond spectrometer geometries, which were used for the high-resolution measurements of the $\mathrm{n}=2 \rightarrow 1$ transitions in highly charged krypton and xenon, respectively (see Chapters 6 and 7 ).

\subsection{Germanium detectors}

Like all solid state detectors, germanium detectors are, basically, semiconductor diodes. A lithium-diffused layer of the germanium single crystal forms the " $n$ contact", i.e., the area where negative charges are enhanced. Ion implantation of boron ions creates the enrichment of positive charges and this layer forms the " $p$ contact". A voltage is applied with such a polarity that the crystal volume between $\mathrm{n}$ - and p-contact is charge depleted and, thus, no DC current can flow through the semiconductor device. Upon impact of an $\mathrm{x}$-ray photon electron-hole pairs are produced in this volume and a current pulse can be detected in the circuit. The number of electron-hole pairs is proportional to the photon energy, which allows the spectral decomposition of the incoming $\mathrm{x}$-ray radiation. In particular, an energy of $2.96 \mathrm{eV}$ is needed to create an electron-hole pair in a germanium crystal which is at a temperature of $77 \mathrm{~K}|78|$. The size of the detectable current pulse, however, also depends on the electrical field strength in the crystal. Therefore, the sensitivity of the detector is proportional to the magnitude of the electrical field strength. The resolution depends 
on the uniformity of the electrical field across the diode. Typically, the magnitude of the electrical field strength in the germanium crystal is around $1 \mathrm{kV}$. Such a field strength requires a cryogenically cooled crystal since too many intrinsic charges are present at room temperature which would yield destructively high currents. Due to the application of cryogenics, crystal and Field Effect Transistor (FET) are embedded in an evacuated aluminum capsule with a beryllium window in the front. A concise compilation of some parameters of the germanium detectors used for measurements presented in this thesis is given in Table 5.1 .

Table 5.1: List of some germanium detectors used at the Livermore EBIT facility. The concise compilation of some detector parameters includes: the area $A_{x t a l}$, and volume $V_{x t a l}$ of the germanium crystal, the thickness of the beryllium window $d_{w i n}$, and the energy resolution measured at $5.9 \mathrm{keV}$. The column ID is for reference purpose only.

\begin{tabular}{|c|c|c|c|c|c|}
\hline Model & $\begin{array}{l}A_{x t a l} \\
\mathrm{~mm}^{2}\end{array}$ & $\begin{array}{l}V_{x t a l} \\
\mathrm{~mm}^{3}\end{array}$ & $\begin{array}{l}d_{w i n} \\
\mu \mathrm{m}\end{array}$ & $\begin{array}{l}\Delta E \\
\mathrm{eV}\end{array}$ & ID \\
\hline EG\&G GLP 16195/10 & 201 & 2011 & 127 & 195 & small GLP \\
\hline PGT IGP 210185 & 210 & 2100 & 127 & 185 & IGP \\
\hline EG\&G GLP 36360/13-P & 1018 & 13232 & 254 & 360 & big GLP \\
\hline EG\&G LOAX 51370/20-P & 2035 & 40696 & 508 & 370 & small LOAX \\
\hline EG\&G LOAX 60495/30-P & 2827 & 84823 & 508 & 495 & big LOAX \\
\hline
\end{tabular}

\subsubsection{Detector efficiency}

The efficiency of the germanium detectors is equal to the probability that the incoming $\mathrm{x}$-ray photon gets absorbed in the charge depleted zone of the germanium crystal. The $\mathrm{x}$ rays have to first penetrate the beryllium window and then either the 600- $\mu \mathrm{m}$ thick n-contact or the $0.3-\mu \mathrm{m}$ thick $\mathrm{p}$-contact. These layers are also called the "dead 
layers" of the germanium crystal. In all the germanium detectors listed in Table 5.1 it is the p-contact which faces the beryllium window. The fraction of $x$-ray photons which penetrate a material of thickness $d$ is given by:

$$
T=\frac{I_{t}}{I_{0}}=\mathrm{e}^{-\mu d}
$$

where $I_{0}$ denotes the incoming and $I_{t}$ the transmitted $\mathrm{x}$-ray intensity, and $\mu$ is the mass attenuation. In most Tables the mass attenuation coefficient is normalized to the density of the material $\rho$, i.e., $\frac{\mu}{\rho}$. The fractional amount of absorption is given by

$$
A=\frac{I_{a}}{I_{0}}=1-\mathrm{e}^{-\mu d},
$$

assuming that none of the incoming $x$-rays are reflected. The mass attenuation represents the overall energy loss of the incoming photon due to interactions with the target material. The main processes contributing to the energy loss of the $\mathrm{x}$-ray photon can be divided into following groups:

- Photoelectric absorption,

- Coherent scattering (Rayleigh scattering),

- Incoherent scattering (Compton scattering), and

- Pair production.

In the photoelectric absorption process the whole energy of the incoming $\mathrm{x}$ ray photon is converted into the binding energy plus the kinetic energy of an originally bound electron, also known as photoelectron. Filling the vacancy created due to this photoionization leads to the emission of either characteristic $\mathrm{x}$ rays or Auger electrons. A rough approximation of the probability of photoelectric absorption for a photon with the energy $E_{h \nu}$ interacting with an atom of atomic number $Z$ is

$$
\tau \sim \text { Const. } \frac{Z^{a}}{E_{h \nu}},
$$


where the exponent $a$ varies between 4 and 5 for [79]. This proportionality, of course, does not account for the characteristic shell structure of the element of atomic number $Z$. The constant (Const.) has the dimensions of Energy ${ }^{3} /$ Length and, thus, the quantity $\tau$ is compatible with the mass attenuation factor $\mu$ given in the equations above. The photoelectric absorption is the dominant part of the $\mathrm{x}$-ray germanium detector interaction considering that for all measurements presented in this thesis the $\mathrm{x}$-ray energy is below $160 \mathrm{keV}$. The coherent scattering or Rayleigh scattering describes the energy loss due to resonant oscillation of the atoms, especially the electrons of the atoms, with the electromagnetic field of the passing $\mathrm{x}$-ray photon. The amount of coherently scattered energy is according to Lord Rayleigh proportional to the photon energy and the refractivity of the medium $|80,81|$. The attenuation due to coherent scattering has the form:

$$
\begin{aligned}
\sigma_{c o h} & =\frac{8 \pi^{3}}{3 N \lambda^{4}}\left(n^{2}-1\right)^{2}= \\
& =\frac{\omega^{4}}{6 \pi N c_{0}^{4}} \frac{N^{2} e^{4}}{m_{e}^{2} \epsilon_{0}^{2}}\left(\frac{1}{\omega_{0}^{2}-\omega^{2}+i \omega \gamma}\right)^{2}= \\
& =\frac{N e^{4}}{6 \pi \epsilon_{0}^{2}}\left\{\begin{array}{c}
1 \\
\left.E_{e^{-}}\left[1-\begin{array}{c}
i \gamma \hbar \\
E_{h \nu}
\end{array}-\left(\frac{E_{0}}{E_{h \nu}}\right)^{2}\right]\right\}^{2}
\end{array} .\right.
\end{aligned}
$$

Only the motion of the electrons is taken into account and, thus, $N$ represents the electron density. The refractivity $n^{2}-1$ was substituted with the term ${ }_{m_{e} \epsilon_{0}}^{N e^{2}} \frac{1}{\omega_{0}^{2}-\omega^{2}+i \omega \gamma}$ |82, 83|. The quantities $\lambda, \omega$, and $E_{h \nu}$ characterize the X-ray photon. $E_{e^{-}}$is the total energy of the coherently oscillating electrons, $m_{e}$ the electron mass, and $c_{o}$ the speed of light. The quantity $\omega_{0}$ describes a resonance frequency of the electron, e.g., the frequency of a dielectronic transition between two bound states, and $\gamma$ is the damping of the electron's oscillation due to collisions. Actually, the sum of all possible resonant transition frequencies should be used instead of only one. For the discussion of the qualitative behavior of the attenuation due to coherent scattering in the domain of $x$-ray energy, however, the use of only one resonance frequency and, thus, only one transition energy $E_{0}$ is sufficient. Assuming that the x-ray energies are far away 
from any of the resonance energies of the medium the amount of coherently scattered photons is mainly determined by the total energy of the electrons. Therefore, the attenuation decreases with increasing $x$-ray energy.

The incoherent scattering can be split into a scattering component (Compton scattering) and an absorption component (Compton absorption). The Compton scattering process describes the partial transfer of the $\mathrm{x}$-ray energy and momentum to an electron. The cross section for Compton scattering can be estimated using the KleinNishina formula which is a fully relativistic approximation for high-energy photons $[84,85]$. Thus, the attenuation - the macroscopic equivalent to the cross section due to this process is

$$
\sigma_{i n c, s}=\frac{N e^{4}}{6 \pi \epsilon_{0^{2}}^{2}} \frac{1}{E_{e^{-}}{ }^{2}\left(1-2 \frac{E_{h \nu}}{E_{e^{-}}}\right)},
$$

with the same notation as in eq.(5.4). The attenuation based on pair production requires a minimum photon energy of $2 m_{0} c_{0}^{2}=1.02 \mathrm{MeV}$. Therefore, this process is not relevant for any of the presented measurements.

Figure 5.1 shows the mass attenuation and the various components according to the discussion above as a function of photon energy for the elements beryllium and germanium. Considering the probability for absorption of the $\mathrm{x}$-ray photon in the beryllium window and the p-contact "dead layer" of the germanium crystal the efficiency of the germanium detector $\eta_{\text {det }}$ is approximately

$$
\begin{aligned}
\eta_{\text {det }} & \approx T_{w i n} \cdot T_{c o n} \cdot A_{x t a l}= \\
& =\mathrm{e}^{-\mu_{B e} d_{w i n}} \cdot \mathrm{e}^{-\mu_{G e} d_{c o n}} \cdot\left(1-\mathrm{e}^{-\mu_{G e} d_{x t a l}}\right) .
\end{aligned}
$$

The indeces win, con, and ${ }_{x t a l}$, stand for window, contact, and crystal, respectively. The fact, that for $\mathrm{x}$ rays within a certain energy interval above the $\mathrm{K}$ edge the attenuation is higher than for the characteristic $\mathrm{K}$-shell $\mathrm{x}$ rays increases the probability for those characteristic $x$ rays to escape the germanium crystal. Thus, the detected energy is reduced by the energy of the escaped photon which is a characteristic quantity of the target material. As a result additional features appear in the detected 


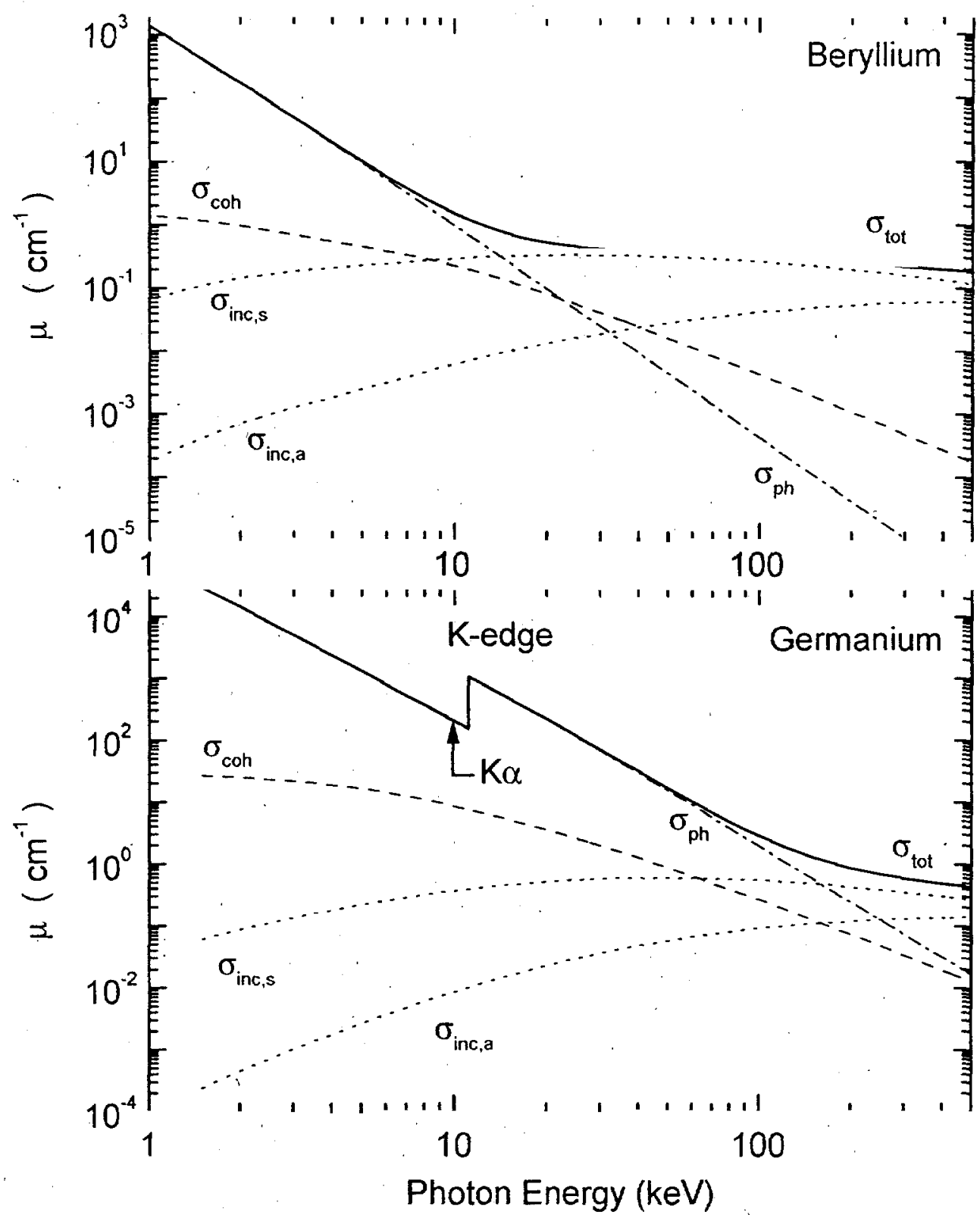

Figure 5.1: Total mass attenuation coefficient $\mu$ for beryllium $\left(\rho=2.34 \mathrm{~g} \mathrm{~cm}^{-3}\right)$ and germanium $\left(\rho=5.32 \mathrm{~g} \mathrm{~cm}^{-3}\right)$ in the domain of $\mathrm{x}$-ray energy. The main contribution are the photoelectric absorption $\sigma_{p h}$ in the low-energy regime and the incoherent scattering (Compton scattering) $\sigma_{i n c, s}$ for high-energy photons. The fraction of the attenuation due to coherent scattering (Rayleigh scattering) is always below $10 \%$. The sharp increase of the germanium attenuation coefficient at $11.10 \mathrm{keV}$ is the $\mathrm{K}-$ edge of this element, i.e., the threshold energy above which it is possible to remove $\mathrm{K}$-shell electrons. The label $\mathrm{K} \alpha$ marks the energy of the characteristic $\mathrm{n}=2 \rightarrow 1$ transition. 
spectrum, the escape peaks. The counts accumulated in the escape peak features are actually missing from the spectral region of the $\mathrm{x}$ ray which caused the initial $\mathrm{K}$-shell photoionization. Therefore, the detector efficiency for this region is reduced by the fraction of characteristic $\mathrm{K}$-shell photons which escape the germanium crystal. A detector efficiency versus x-ray energy plot is shown In Figure 5.2 the detector efficiency is plotted versus the energy of the detected $x$-ray photons. For high-energy $\mathrm{x}$-rays the detector efficiency mainly depends on the depth of the germanium crystal. The K-edge of the germanium mass attenuation coefficient seen in Figure 5.1 does not appear in the detector efficiency curve due to the thickness of the germanium crystals. In fact, it only takes a 300- $\mu \mathrm{m}$ thick layer of germanium to absorb $99 \%$ of all $\mathrm{x}$-rays with an energy just below the germanium $\mathrm{K}$-edge, i.e., just below $11.1 \mathrm{keV}$. However, right above the $\mathrm{K}$ edge the detector efficiency shows a little dip. The efficiency drop is caused by the increased probability for characteristic $\mathrm{K}$-shell $\mathrm{x}$-rays to escape the germanium crystal since the mean absorption depth of $\mathrm{x}$ rays with energies

slightly above the $\mathrm{K}$ edge is much smaller than of characteristic $\mathrm{K}$-shell $\mathrm{x}$ rays. In the low-energy regime the efficiency is dominated by the thickness of the beryllium window. The design of the window has to withstand the pressure difference between the evacuated capsule and the outside pressure. Thus, the window thickness and the crystal size are correlated parameters.

\subsubsection{Detector resolution and apparatus profile}

The detector resolution is determined by the jitter in the current pulse which is detected upon impact of $\mathrm{x}$-rays of constant energy. This jitter depends on the $\mathrm{x}$-ray energy. For photon energies $(E)$ up to $1.5 \mathrm{MeV}$, the following approximate expression is useful for predicting the resolution of a germanium detector:

$$
\begin{aligned}
& \Delta E=\sqrt{ } N^{2}+\xi E \\
& \text { with } \quad \xi=2 \mathrm{eV} .
\end{aligned}
$$




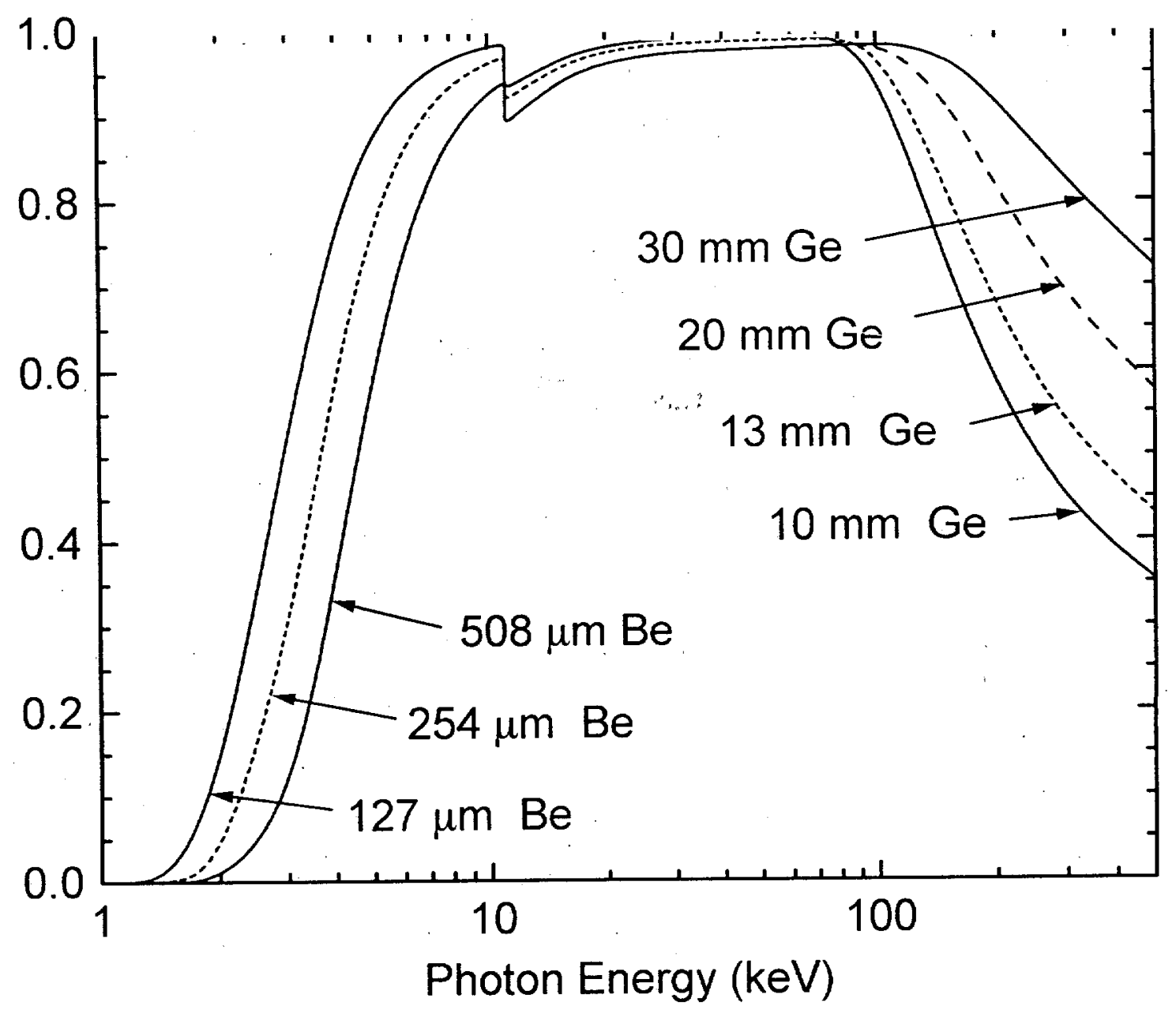

Figure 5.2: Efficiency of the germanium detectors listed in Table 5.1 in the domain of the $\mathrm{x}$-ray energy. The absorption probability of a low-energy $\mathrm{x}$-ray is dominated by the thickness of the beryllium window. The efficiency in the high-energy regime is determined by the thickness of the germanium crystal. The dip in the detector efficiency right above the $\mathrm{K}$ edge of germanium is due to the increased escape probability of characteristic $\mathrm{K}$-shell $\mathrm{x}$ rays.

The energy resolution $\Delta E$ is defined as the full width at half maximum (FWHM) of the spectral line. The noise line width $N$ describes the minimum line width achievable and, thus, is a detector specific quantity. The noise line width includes the dependence of the resolution on the impurities in the germanium crystal, which affect the energy required to create an electron-hole pair in the crystal, and on the spatial distribution 
of the electrical field strength across the crystal volume. Furthermore, the detector resolution is sensitive to changes in the temperature of the crystal, and to fluctuations in the count rate. These factors affect the energy required for an electron-hole pair production in the germanium crystal, and the mobility of the charges in the crystal. Electronic noise in the data acquisition process adds to the jitter in the current pulse signal, too. The energy resolution of the germanium detectors listed in Table 5.1 was calculated using eq.(5.7) and the result is shown in Figure 5.3 together with the resolving power, $\frac{E}{\Delta E}$.

Within the energy regime of interest for atomic physics such a resolving power is not sufficient for measurements involving the line profile characteristic to the observed $\mathrm{x}$-ray source. The appearance of all observed lines is dominated by the apparatus profile. This apparatus profile of the germanium detector is asymmetric combining the statistical, thus, symmetric broadening of the line due to electronic noise and charge production statistic in the germanium crystal with asymmetric components, i.e., a low-energy tail due to field strength reduction caused by the produced charges and incomplete charge collection in the germanium crystal, and a higher background at the low-energy side of the observed line due to escape peaks. The probably best description of the actual peak shape has been developed by Gunnick et al. $[86,87,88]$ here at the LLNL ${ }^{24}$. Accounting for the symmetric and asymmetric components their line profile has following analytic form:

$$
f(x)=y_{0} e^{-\frac{(x-\bar{x})^{2}}{2 \sigma^{2}}}+a y_{0} e^{b(x-\bar{x})}\left[1-e^{-\frac{c(x-\bar{x})^{2}}{2 \sigma^{2}}}\right] H(\bar{x}-x),
$$

where $\bar{x}$ is the line centroid with the amplitude $y_{0}$, and $a, b$, and $c$ are the free parameters for fitting the low-energy tail. $H$ is the Heaviside function which takes on the value 1 for positive arguments, i.e., $\bar{x}-x>0$, and 0 otherwise. Thus, the second term only contributes to the low-energy side. A somewhat refined version of this profile was incorporated in a software package written by D.A. Knapp, and used for

\footnotetext{
${ }^{24} \mathrm{~A}$ concise discussion of this line profile can be also found in Ref. [89] which might be easier accessible than the technical report cited above
} 


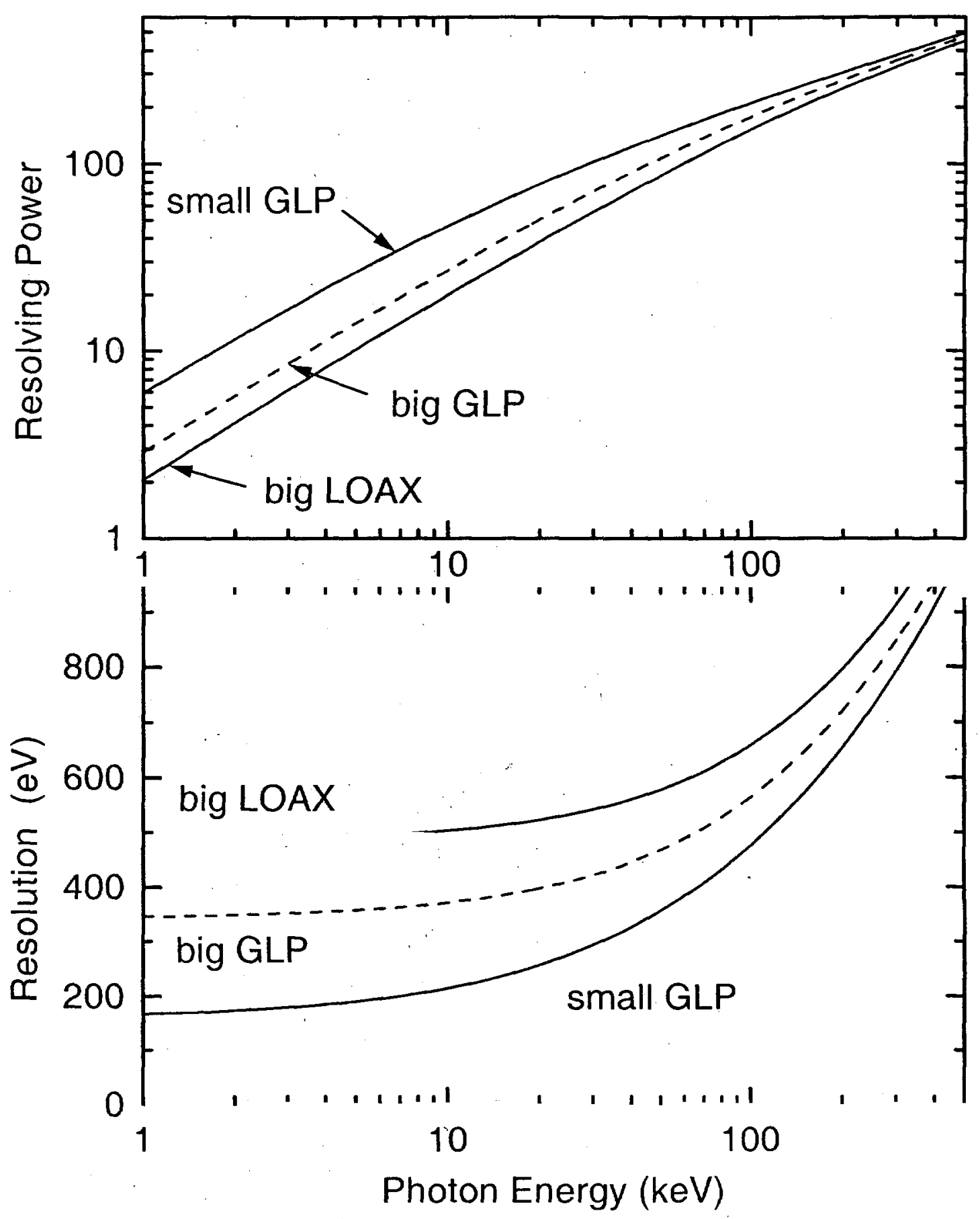

Figure 5.3: Intrinsic resolution and resolving power of some germanium detectors listed in Table 5.1 in the domain of the $\mathrm{x}$-ray energy. 
the analysis of all spectra taken with a germanium detector, e.g., the measurement of the xenon Ly- $\alpha_{1}$ transition energy presented in Section 7.2, page 175.

The germanium detectors are the work horses at the Livermore EBIT facility. Their broad spectral range makes them the perfect tool for performing a spectral survey of the $\mathrm{x}$-ray radiation emitted by the trapped ions. Additional advantages are the easy setup and the transportability of these detectors.

\subsection{High-resolution crystal spectrometer}

In crystal spectrometers the internal crystal structure is used for spectral decomposition of the observed $\mathrm{x}$-ray radiation by means of diffraction. Thus, crystal spectrometers are wavelengths selective instruments, in contrast to the solid state detectors where energy selective principles are applied for spectrally resolving the incoming radiation. Since the first experimental verification of the diffraction of $x$-ray $[90]$ and the first successful implementation of crystals for spectroscopic purposes [91], a large amount of different types of crystal spectrometers has been developed. The high resolution generally achievable with these diffraction instruments, however, limits the band width of simultaneously observable x-ray energies. Additionally, the efficiency is usually lower for crystal spectrometers compared to solid state detectors.

The following Sections focus on only two spectrometer geometries, i.e., the von Hámos reflection-type geometry $|92,93|$ and the DuMond transmission-type geometry [94|. Both geometries are based on a cylindrically bent crystal design. The von Hámos type spectrometer requires position sensitive detection of the diffracted $\mathrm{x}$ rays, which is not the case for the DuMond type setup where the spectrometer is used as a monochromator. Therefore, the brief overview of the type and properties of the position sensitive detector is organized as a part of the von Hámos spectrometer section. Before describing the properties of the spectrometers a brief introduction to the theory of $x$-ray diffraction is given. 


\subsubsection{X-ray diffraction}

Following the suggestions of Ewald [95] the theory of $x$-ray diffraction can be divided into four stages regarding the depth of treatment, i.e., the amount of various physical processes which are accounted for:

- the geometrical theory,

- the kinematical theory,

- the dynamical theory, and

- the quantum theory of diffraction.

In the simplest approach, the geometrical theory, the crystal is considered as a perfect infinite three dimensional structure. Calculating the difference in the optical path between the scattered perfectly monochromatic $x$-ray wavelets allows to determine the directions under which the wavelets constructively interfere and, thus, the diffracted $\mathrm{x}$ rays appear. Considering only one specific lattice orientation and only one $\mathrm{x}$-ray wavelength determines completely the direction of incidence and scatter with respect to the lattice planes defined by the given orientation. The relation between the wavelength $\lambda$, the angle of incidence $\theta$ and the distance between the lattice planes $d$ is given by Bragg's law [91], which has the form:

$$
n \lambda=2 d \sin \theta
$$

with $n$ being the order of diffraction. Including the dependence of the index of refraction on the $\mathrm{x}$-ray energy modifies Bragg's law to $[96]$ :

$$
n \lambda=2 d_{\infty}\left[1-\frac{\delta}{\lambda^{2}}\left(\frac{2 d_{\infty}}{n}\right)^{2}\right] \sin \theta,
$$

where $d_{\infty}$ denotes the lattice spacing at $\mathrm{x}$-ray energies far above any resonance line of the crystal, $n$ the order of diffraction, $\delta$ the deviation of the refractive index $\mu$ from unity, i.e., $\delta=1-\mu, \lambda$ the wavelength of the $\mathrm{x}$ rays in air, and $\theta$ the Bragg angle. 
Note, that instead of varying the wavelength according to the index of refraction this equation introduces a variable lattice spacing which depends on the wavelength and order of diffraction. By comparing eq.(5.9) and eq.(5.10) this variable lattice spacing can be expressed as a function of the order of diffraction and the wavelength, i.e.,

$$
2 d_{n, \lambda}=2 d_{\infty}\left[1-\frac{\delta}{\lambda^{2}}\left(\frac{2 d_{\infty}}{n}\right)^{2}\right] .
$$

Usually, the factor $\delta$ is much smaller than one and, thus, the variable lattice spacing $2 d_{n, \lambda}$ is a only slowly changing function with respect to lambda. An observable impact of the effect caused by the index of refraction on the $2 \mathrm{~d}$ spacing has only been encountered when comparing spectra which were taken at different orders of diffraction (see Section 6).

The next stage, the kinematical theory, accounts for the spatial limitation of the crystal structure. This modifies the spatial frequency of the scattering centers, i.e., the atoms, allowing more relaxed conditions with respect to the directions of diffraction. Thus, these directions are defined by an angle distribution $\theta \pm \Delta \theta$ instead of only a single angle. Quantities like the resolving power of the crystal become accessible with this approach. The kinematical theory, however, still lacks some fundamental aspects, such as conservation of energy. This problem of energy transfer from the incident $x-$ ray beam onto the crystal and to the various reflection beams or transmission beams is addressed by the dynamical theory. The quantum theory of diffraction, too, includes the conservation of energy since the $x$-ray radiation field and the state of the crystal are considered as parts of one single system. Balancing the total energy allows an estimate of the crystal's reflectivity, for example.

\subsubsection{Reflection-type spectrometer von Hámos geometry}

The crystals employed in reflection-type spectrometers are cut in such a way that the lattice planes of interest are parallel to the crystal surface, i.e., the front side of the crystal. The incoming and the diffracted $\mathrm{x}$ rays are, thus, in the same half space with 
respect to the crystal surface. The reflection-type geometry was the geometry of the prototype of crystal spectrometers developed by the Bragg's ${ }^{25}$ [91]. The advancement in the von Hámos design $\{92,93\}$ is the implementation of a cylindrically curved instead of a flat crystal, where the curvature of the crystal is along the "non-dispersive" direction, i.e., the direction given by the trajectories of constant Bragg angle. The axis of the cylinder, which describes the crystal's curvature, defines the optical axis of the von Hámos geometry. Therefore, the center of the spectrometer entrance slit and the center of the detector should be a part of this axis. The principal setup of a von Hámos spectrometer is shown in Figure 5.4. With the crystal curved along the non-dispersive direction an image of the slit is obtained at location of the optical axis. This enhances the intensity tremendously in comparison with a flat-crystal spectrometer. The von Hámos design, thus, opens the window on high-resolution measurements since the detected intensity is no longer indirect proportional to the square of the entrance slit to detector distance.

Due to the special source geometry of EBIT and SuperEBIT, i.e., the 12.7-mm high and less than $100-\mu \mathrm{m}$ wide accessible electron beam trap region, no entrance slit is required for the implementation of a von Hámos spectrometer since the trap region itself qualifies as a slit. Although the trap region is imaged onto the plane defined by the electron beam and the optical axis the standard von Hámos spectrometer setup at the EBIT facility is based on a detection of the diffracted $x$-rays within a plane which is basically perpendicular to the incoming $x$-rays. This modification is necessary due to the use of a gas-filled proportional counter instead of an x-ray film. The gas-filled detector, however, has a significant depth and, therefore, the "correct" setup would introduce a large decrease in the resolving power due to the parallax effect. A typical von Hámos setup at the EBIT facility is sketched in Figure 5.5. Using the notation introduced in this Figure, the angular range observable with this

\footnotetext{
${ }^{25}$ Father William Henry and son William Lawrence Bragg;
} 


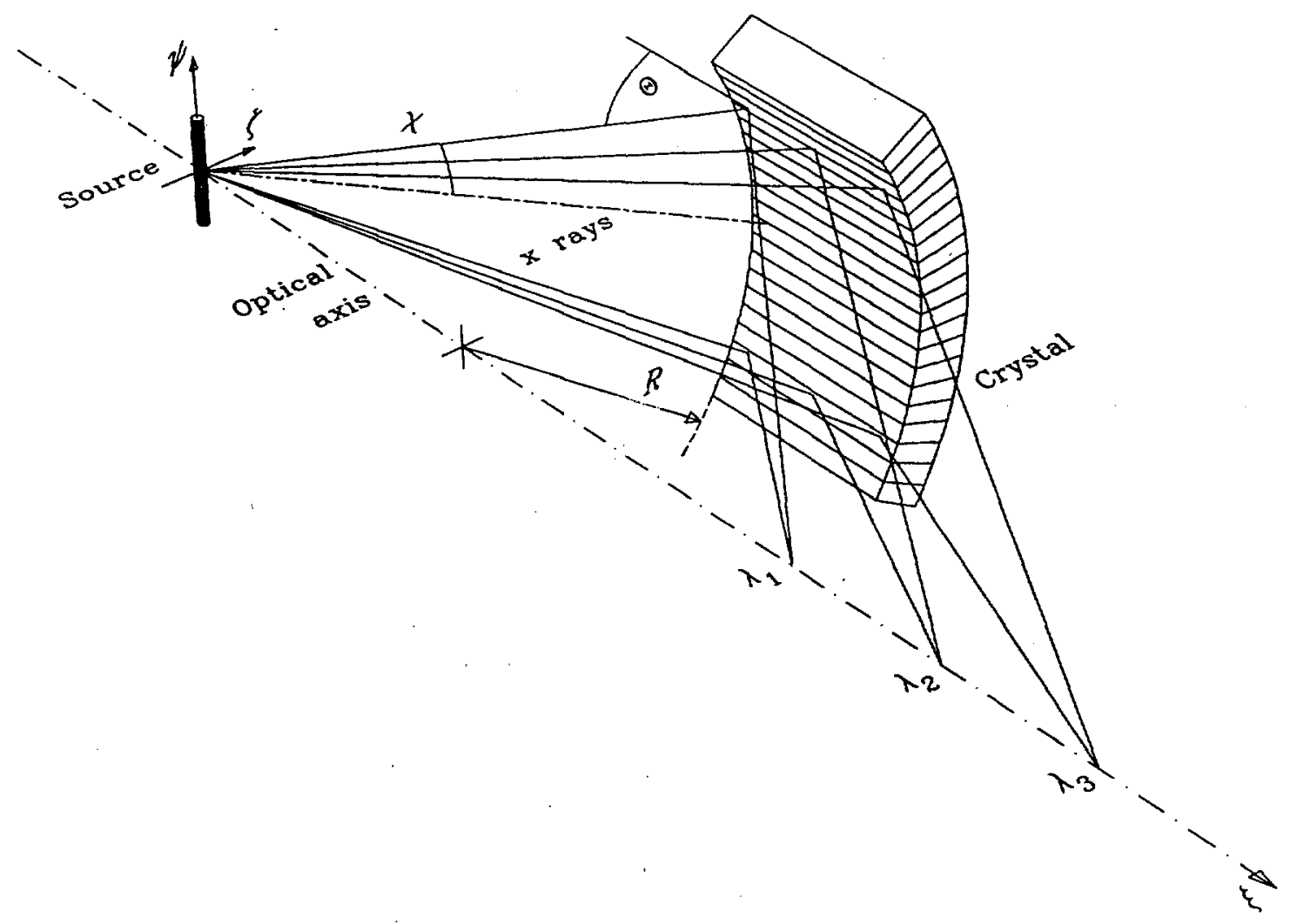

Figure 5.4: The axis of the cylinder which describes the curvature of the crystal (radius $R$ ) is the optical axis of the von Hámos spectrometer setup. All $\mathrm{x}$ rays emitted form the point where the source intersects the optical axis $(\xi=\psi=\zeta=0)$ hit the crystal at a Bragg angle $\theta$ which is solely determined by the distance between the source point and the crystal surface and, thus, by the coordinate $\xi$ of the $x$-ray impact position. The wavelength-selective images of this point are formed along the optical axis. For an extended source, however, the Bragg angle depends on the angle $\chi$ and the image becomes distorted. These aberrations are smallest if the source extends in the "non-dispersive" direction as sketched in this Figure.

spectrometer is either

$$
\tan \left(\theta_{\max }-\theta_{\min }\right)=\frac{4 w_{x} R \sin \theta_{0}}{4 R^{2}-w_{x}^{2} \sin ^{2} \theta_{0}},
$$

assuming that the limitation is given by the width of the crystal $w_{x}$, or

$$
\tan \left(\theta_{\max }-\theta_{\min }\right)=\frac{2 w_{d} R \sin \theta_{0}}{4 R^{2}-w_{d}^{2} \sin ^{2} \theta_{0}},
$$




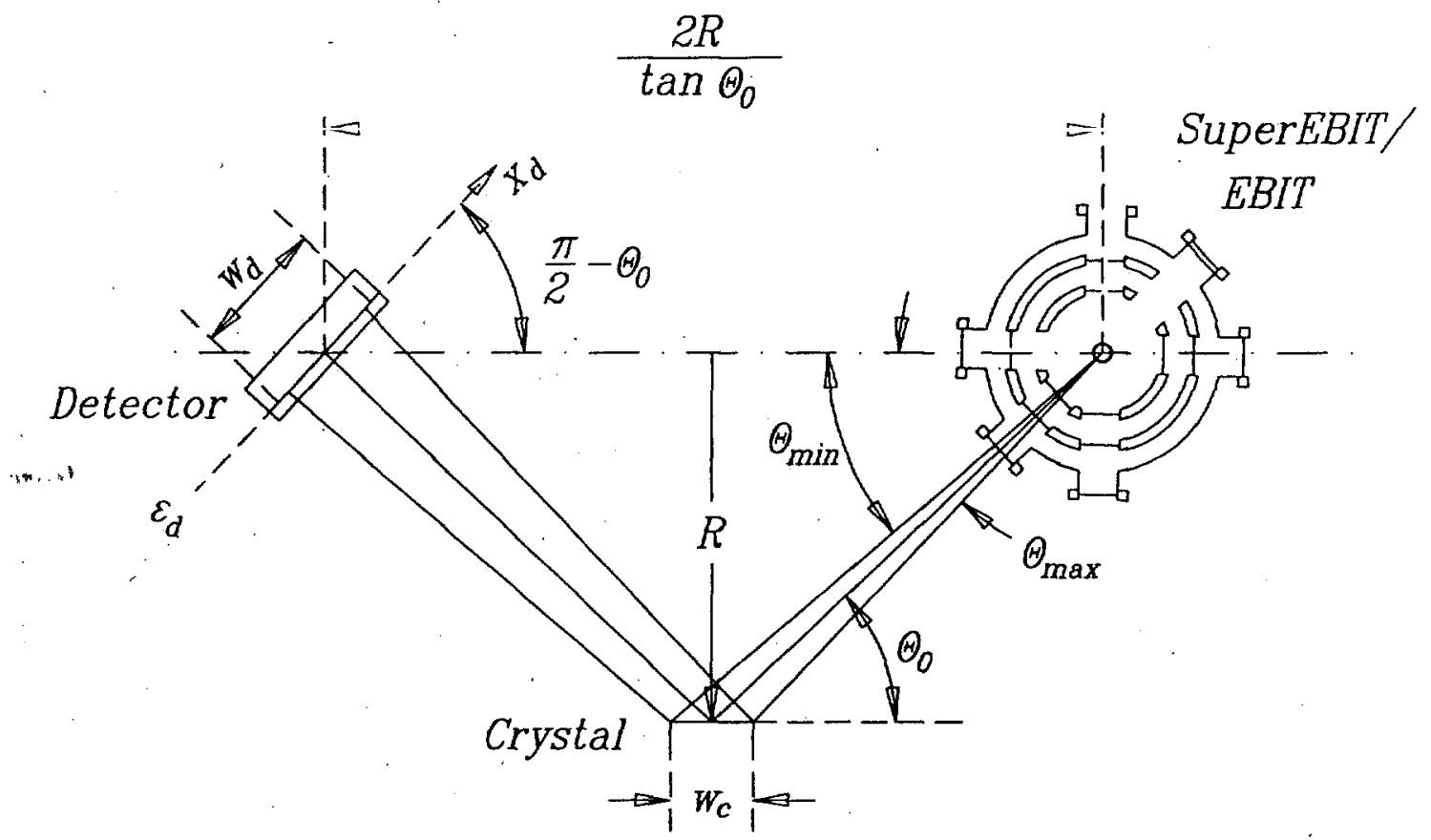

Figure 5.5: A sketch of the EBIT/SuperEBIT device is shown in a cross section with the electron beam in the center of the device and perpendicular to the page. Geometric quantities important to the von Hámos spectrometer setup are: the radius of the crystal curvature $R$, the various Bragg angles $\theta$, and the crystal and the detector width $w_{c}$ and $w_{d}$, respectively. The detector plane $\varepsilon_{d}$ and the orientation of the spatially resolved direction $x_{d}$ are used for the determination of the imaging properties of the von Hámos geometry.

when the limitation is due to the width of the detector window. The position sensitive detectors used for the von Hámos spectrometers at the EBIT facility have a window which is twice as wide as the crystals and, thus, the angular range is always limited by the crystal width: The minimum and maximum Bragg angle achievable with this setup are

$$
\tan \theta_{\text {min, }, \text { max }}=\frac{\tan \theta_{0}}{1 \pm \frac{w_{x}}{2 R} \tan \theta_{0}},
$$

which together with the $2 \mathrm{~d}$ spacing of the crystal gives the minimum and maximum wavelength of the observable $\mathrm{x}$-ray band according to eq.(5.9).

For the determination of the dispersion the two quantities $x_{d}$ and $\varepsilon_{d}$ are introduced, 
where the first just defines the orientation of the spatially resolved direction and $\varepsilon_{d}$ denotes the detector plane as seen in Figure 5.5. The Bragg angle $\theta$ of a line detected at the location $x$ on the detector is

$$
\theta=\theta_{0}+\arctan \left(\frac{x-x_{0}}{2 R} \sin \theta_{0}\right)
$$

where $\theta_{0}$ is the Bragg angle of the $\mathrm{x}$-ray beam which would be perpendicular to the position sensitive detector plane $\varepsilon_{d}$ and would hit the detector at the position $x_{0}$. For crystals with a large radius of curvature with respect to the spatial separation of the lines in the detector plane, i.e., $2 R \gg\left(x-x_{0}\right)$, the angular dispersion becomes a linear function of the line position $x$ on the detector. The dispersion with respect to the wavelength involves again the sine function and, therefore, is usually not linear in the domain of $x$. Ideally, there are only two calibration lines necessary for determination of the parameters $\theta_{0}$ and $x_{0}$. The measured uncertainties, however, have a significant impact on the solution for these parameters and the use of more calibration lines is always advisable.

The resolving power of the von Hámos spectrometer depends on several components which include the spatial resolution of the detector, the resolving power of the crystal, the spatial extension of the $\mathrm{x}$-ray source, and the imaging aberrations due to the spatial extension of the $x$-ray source. The imaging aberrations have a decreasing impact on the resolving power especially, if the detector can only resolve one spatial dimension, as is the case for all position sensitive proportional counters at the Livermore EBIT facility. A detailed discussion of the resolving power in connection with a measurement is presented in Section 6.1.1.1, and only the imaging aberrations are considered in following. The imaging properties derived by von Hámos |92| allow the calculation of the location of the imaged point with respect to the direction given by the optical axis $\xi$ as a function of the source point position $\psi$ and $\zeta$ (see Figure 5.4 for the geometric assignment of these coordinates). Assuming that the source is part of the $\psi$ axis, i.e., $\xi=\zeta=0$ for all source points, the distance $\xi$ at which the image 
appears is

$$
\xi=\frac{2(R-\psi \sin \chi)}{R-2 \psi \sin \chi} \sqrt{\left(\frac{R-y \sin \chi}{\tan \theta_{0}}\right)^{2}-\psi^{2} \cos ^{2} \chi}
$$

where $0 \leq 2 \chi \leq \pi$ is the polar opening angle of the crystal seen from the optical axis. ${ }^{26}$ Figure 5.6 shows the deviation in the distance $\xi$ with respect to the image of the source center $(\xi(\psi)-\xi(0))$ as a function of the height of the source point $\psi$ for several different radii $R$. The accessible height of the electron beam region is marked with dashed lines. Using a detector with only one dimension of spatial resolving power introduces a line spread due to the integrated measuring technique along the nonresolving dimension. The calculations which were performed assuming Bragg angles of $28^{\circ}$ and $45^{\circ}$ show that the line spread caused by the imaging properties of the von Hámos geometry can is below the $100 \mu \mathrm{m}$ regime for most arrangements, except for the $30-\mathrm{cm}$ curvature crystal geometry, where the maximum deviation from the image of the source center is $130 \mu \mathrm{m}$ at a $45^{\circ} \mathrm{Bragg}$ angle. This deviation is symmetric with respect to the optical axis and, thus, the imaging properties of the von Hámos geometry introduce an asymmetric line broadening, which strongly depends on the radius of curvature of the crystal and less strongly on the Bragg angle.

However, the discussion about the resolving power of the von Hámos spectrometer in context with the measurement of the $\mathrm{K} \alpha$ radiation of highly charged krypton in Section 6.1.1.1 reveals that the source image aberrations are only of minor importance and is much less than, for example, the line broadening caused by the spatial resolution of the detector, discussed below.

\subsubsection{Position-sensitive proportional counter}

The von Hámos geometry requires position sensitive detection of the diffracted $\mathrm{x}$ rays. The low $\mathrm{x}$-ray flux at EBIT/SuperEBIT, however, makes it an experimental

\footnotetext{
${ }^{26}$ In the original version [92] the radius $R$ is set equal to one. Note, that the quantity $y_{1}$ in von Hámos's paper is treated as a negative number and, thus, at the beginning of eq.(1) it should state $\overline{O P^{\prime}}=-y_{1}$.
} 

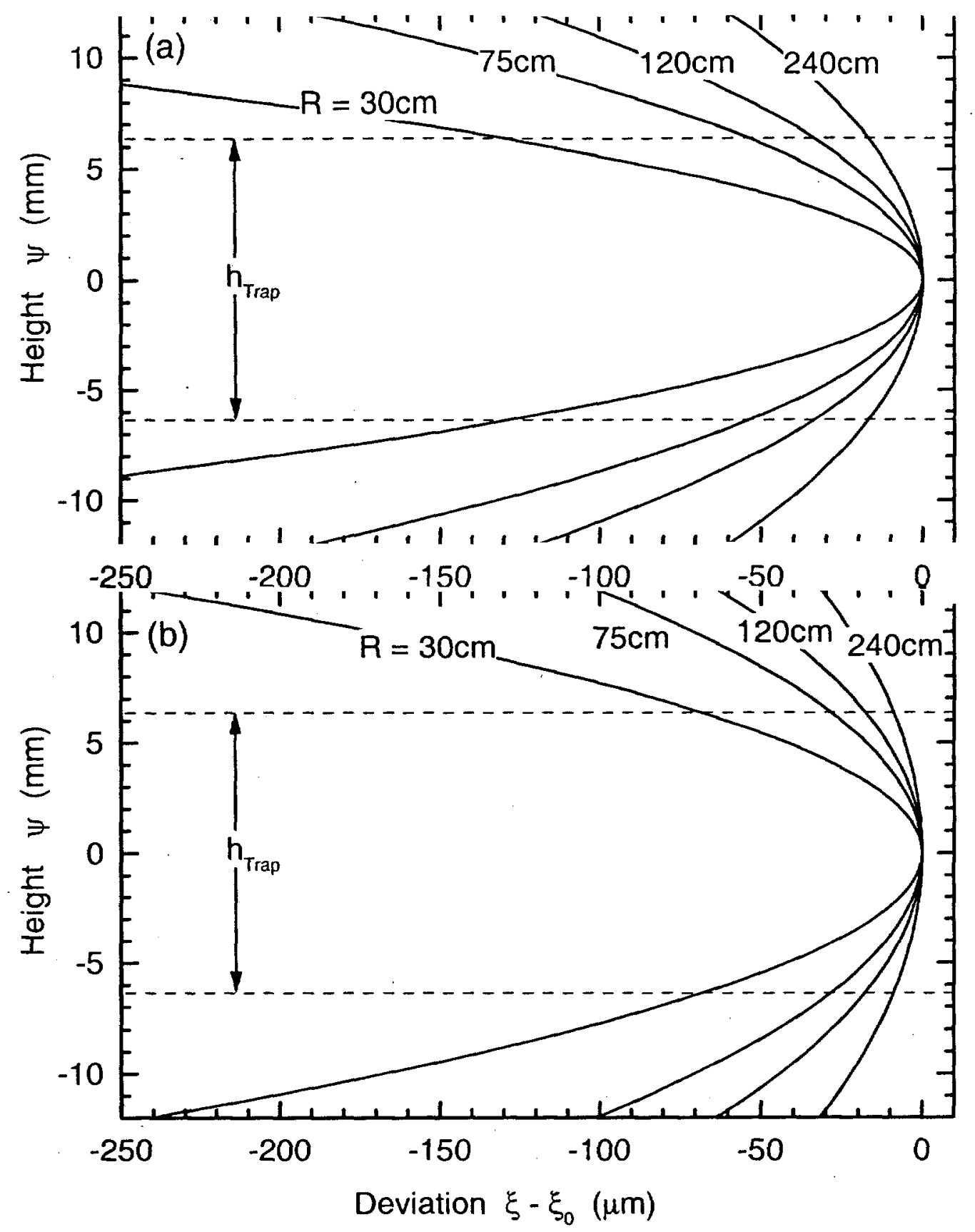

Figure 5.6: Position of the imaged source points with respect to the image of the source center, $\xi(\psi)-\xi(0)$. The deviation from the ideal position is plotted as a function of the vertical distance from the optical axis. The graphs were rotated for better visualization of the effect of the vertical extension of the $\mathrm{x}$-ray source, i.e., the hight of the electron beam trap region $h_{\text {Trap. }}$. The image positions were calculated according to eq.(5.16) with Bragg angles of (a) $45^{\circ}$ and (b) $28^{\circ}$. The radii of curvature are the different radii currently available at the Livermore EBIT facility. 
challenge to find the fine line of sufficient resolution, optimized counting efficiency, and low background. The detector type best suited and most flexible with respect to this compromise is a gas-filled proportional counter.

The detection mechanism is based on the ionization of an atom or molecule of the gaseous medium in the detector due to photon impact. This is equivalent to the photoelectric absorption in the solid state detectors discussed under Section 5.1.1 above. The ionization probability depends, like the probability of photoelectric absorption, strongly on the atomic number of the elements in the gas with which the $\mathrm{x}$-ray photon interacts. Therefore, the noble gas elements are the preferable choice. Having a gas which is chemically inert with respect to all substances used in the detector design is an additional advantage. The much lower density of the gaseous medium in the proportional counters versus the solid state crystal in the germanium detector yields a much lower attenuation of the incoming $x$ rays assuming similar geometric extensions, of course. Additionally, the efficiency loss due to the escape of characteristic innershell $\mathrm{x}$-rays emitted by the photoionized gas atoms.is more pronounced than in the solid state detectors Compensating the lower attenuation by increasing the detector volume would lead to a decrease in the spatial resolution achievable. Increasing the gas density, however, improves the detection efficiency while keeping the detector dimensions constant.

The detection of the $\mathrm{x}$-ray photon is, thus, converted into the detection of the ionization products, i.e., the ion and especially the photoelectron. The electrons and ions are accelerated by an electrical field which is applied across the gas-filled detector volume. The electrons gain enough kinetic energy to ionize more neutral gas particles on there way to the anode which is an array of thin wires. The magnitude of this avalanche of electrons is very sensitive with respect to the electrical field strength and, thus, the high voltage which is applied to the detector's electrodes. Admixing'a so called quenching gas, e.g., methane or isobutylene, provides some internal protection against the startup of self sustaining discharges that could destroy the extremely 
thin anode wires of the detector. The controlled micro discharge created after initial photoionization is a detectable current pulse, and the amplitude of this pulse is proportional to the energy of the ionizing $x$-ray photon, assuming that the current pulse profile is a constant quantity. Due to this electron avalanche the distribution of the electric field strength is not as homogeneous as in the case of the germanium detectors. Therefore, the pulse in a gas-filled detector has a much broader response with respect to the pulse hight signal, and the resolving power of the gas-filled detector as a spectrometer by itself is much lower than that of the germanium detectors. However, the resolving power is sufficient for using the pulse height distribution of the detected signal as a tool for the suppression of background counts, i.e., the low-energy noise counts and the very high-energy pulses generated by gamma rays.

The design of the position sensitive proportional counters used with the von Hámos crystal spectrometer is similar to the "Källne" detector [97] which was designed for soft- $\mathrm{x}-$ ray applications. Several modifications have been made allowing the efficient detection of higher $\mathrm{x}$-ray energies [98], e.g., the $13-\mathrm{keV}$ photon energies emitted by $\mathrm{n}=2 \rightarrow 1$ transitions in heliumlike krypton (see Section 6.1 ). The proportional counter has an active volume of $(95 \times 30 \times 4) \mathrm{mm}^{3}$ and is filled with $70 \%$ (by volume) xenon and $30 \%$ methane. The pressure in the detector is 5.38 bar (78 psig) above atmosphere. After three purging cycles, i.e., carefully filling and releasing the gas, the amount of residual gases in the detector is below $1 \%$, and the final xenon-methane gas pressure is roughly 6.4 bar. The high pressure requires a 1-mm thickness of the beryllium window which is mounted on a stainless steel lid that includes a support structure for the $(95 \times 30)-\mathrm{mm}^{2}$ window. This support structure is a set of three horizontal ribs $1.5 \mathrm{~mm}$ in height which block out $15 \%$ of the incident $\mathrm{x}$ rays.

The anode consists of eight parallel very thin gold wires and is placed exactly between the cathode and the beryllium window. The latter acts like the cathode on ground potential. The symmetric arrangement is crucial for the homogeneity of the electrical field across the active detector volume. The key to the position sensitivity is 
a cathode delay line etched on a printed circuit board. When the electron avalanche hits the anode wires it induces a mirror charge at the equivalent position of the cathode. Both ends of the anode and of the cathode are connected to the power supply and signal processing units, respectively. Thus, the current pulse travels in both directions. The time difference between the arrival of the first "half pulse" at one end of the delay line and the second "half pulse" at the other end depends on the location of the electron avalanche impact. Due to the delay line design of the cathode electrode the time difference becomes a comfortably detectable quantity. In particular, it takes about $160 \mathrm{~ns}$ for the pulse to travel from one end to the other end of the delay line. Basically, the longer each loop $^{27}$ of the cathode delay line and the narrower the path on the printed circuit board, the better the spatial resolution. The spatial resolving power of the current proportional counter design is, actually, limited by the width of the electron avalanche at the location of the anode wires. This final size of the micro discharge is proportional to the gas pressure and indirectly proportional to the strength of the electrical field. It also depends on the spatial distribution of the photoelectrons initially created by the $x$-ray impact. The range of the photoelectrons increases with x-ray energy but decreases with gas pressure.

For the determination of the detector's spatial resolution a $51-\mu \mathrm{m}$ slit collimator was designed with a special mount for $\mathrm{x}$-ray sources, contained in a 5-mm diameter capsule. The collimator is attached to a high-precision translation stage allowing the characterization of the detector resolution as a function of the position and the determination of the spatial dispersion of the experimental setup. Figure 5.7(a) shows the result of such a measurement, i.e., the narrow lines detected from a ${ }^{55} \mathrm{Fe}$ source mounted on the back of the collimator as a function of collimator position with respect to the dispersive (horizontal) direction of the detector. The widths of the recorded lines range from a minimum of $145 \mu \mathrm{m}$, in the center, to $200 \mu \mathrm{m}$ at the edges of the active detector area. The intrinsic width of the lines, i.e., the width of the colli-

\footnotetext{
${ }^{27}$ The loops are perpendicular to the anode wire array for optimized spatial resolution.
} 
mated beam at the position of the anode wires, is $120 \mu \mathrm{m}$. The detector resolution is determined by the deconvolution of measured and intrinsic profile. A simple approximation by assuming Gaussian line profiles yields a detector resolution of $80 \mu \mathrm{m}$, in the center, to $160 \mu \mathrm{m}$ at the edges. This is about two times better than the spatial resolution of the low-pressure version $(\approx 1$ bar over pressure) |57|. A comparison between the high-pressure and the low-pressure version of the proportional counter with respect to some characteristic parameters is given in Table 5.2 .

Table 5.2: Comparison of some characteristic parameters of a position sensitive proportional counter as a function of gas pressure. The detector has an active volume of $(95 \times 30 \times 4) \mathrm{mm}^{3}$ and is filled with $70 \%$ (by volume) xenon and $30 \%$ methane. The parameter "over pressure" lists the pressure above one atmosphere. The values for the low pressure version were measured by Dave Vogel [57]. The efficiency value accounts also for the absorption in the beryllium windows.

\begin{tabular}{rcc}
\hline parameters & high & low \\
\hline over pressure (bar) & $5.38(78 \mathrm{psig})$ & $1.1(16 \mathrm{psig})$ \\
Be window $(\mathrm{mm})$ & 1.0 & 0.127 \\
Anode voltage $(\mathrm{kV})$ & 4.5 & 2.3 \\
spatial resolution $(\mu \mathrm{m})$ & $80-160$ & $155-300$ \\
efficiency at $13 \mathrm{keV}(\%)$ & 55 & 20 \\
\hline \hline
\end{tabular}

The spatial dispersion was determined by evaluating the line centroid positions as a function of the position of the high-precision translation stage. A deviation from the expected linearity is an important piece of information for accurate spectroscopic measurements. The measured spatial dispersion is actually the dispersion of the whole setup, including the signal processing equipment. The measured deviations from exactly linear behavior - plotted in Figure 5.7(b) - show a maximum value of $25 \mu \mathrm{m}$ at the edge of the active area. The overall deviation from linearity is 
$0.1 \%$. Another important property is the uniformity of the detector response with respect to the efficiency. This detector characteristic was measured by placing a non collimated ${ }^{55} \mathrm{Fe}$ source one meter from the detector window, along the line of sight, i.e., perpendicular to the window. The recorded intensity profile represents the spatially resolved counting efficiency. The result displayed in Figure 5.7(c) shows two non uniformities around channel numbers 825 and 1050. These $10 \%$ variations from the ideal flat response are due to impedance mismatches along the strips of the cathode delay line. Thus, magnitude and location of these dips are characteristic to each cathode board.

For implementation of the gas-filled detector in long time experiments continuous maintenance regarding the composition of the detector gas is necessary. Due to outgasing of all parts inside the detector, the fractional amount of the desired detector gas mixture decreases over time, especially for low-pressure detectors. The change in the gas composition usually reduces the number of photoelectrons created upon impact of an $\mathrm{x}$-ray photon of given energy and, thus, the detected pulse height. The pulse height distribution, however, is used for the suppression of background counts by setting gates around the $\mathrm{x}$-ray energy, i.e., the pulse height, of interest. Therefore, a shift in the pulse height response of the detector might significantly distort the signal to noise ratio and perhaps line intensity ratios. Periodically purging and refilling the detector keeps this systematic uncertainty at a minimum. In fact, most of the detectors have a "built-in" purging an refilling mechanism due to unavoidable small gas leaks.

\subsubsection{Transmission-type spectrometer DuMond geometry}

Successful implementation of high-resolution crystal spectrometer for observation of $\mathrm{x}$-ray radiation of energies in the $30-\mathrm{keV}$ range and higher requires a different spectrometer type - different from the von Hámos and flat-crystal spectrometers based on reflection of the $\mathrm{x}$-rays - due to the low $\mathrm{x}$-ray flux emitted by the SuperEBIT 

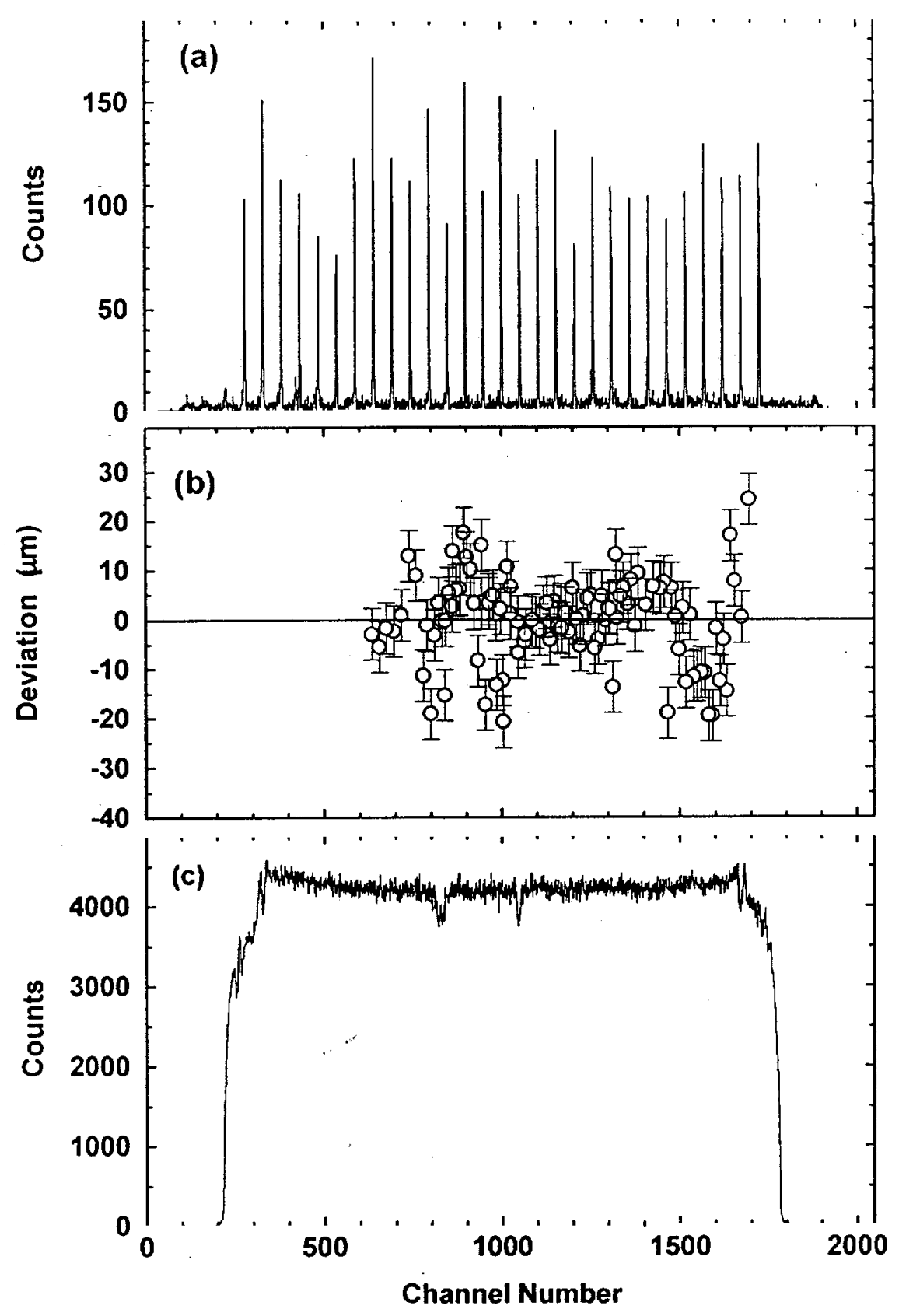

Figure 5.7: Spatial resolution, linearity, and uniformity of the gas-filled position sensitive detector response. The spectrum (a) represents the sum of a series of measurements with a collimated ${ }^{55} \mathrm{Fe}$ source mounted on a high-precision translation stage. The deviation of the fitted line centroid positions from linear behavior with respect to the translation stage readout is shown in (b). Placing the non collimated ${ }^{55} \mathrm{Fe}$ source one meter from the detector window yielded spectrum (c). The dips around channel numbers 825 and 1050 are due to impedance mismatches along the strips of the cathode delay line of the detector. 
device, which basically prohibits the use of higher orders of diffraction. Besides the reduced crystal reflectivity at small Bragg angles, crystal spectrometers that depend on spatially resolved detection of the diffracted $\mathrm{x}$ rays also suffer from the tremendous decrease in efficiency of the position sensitive detectors at higher $x$-ray energies |98|. Thus, for the hard $x$-ray regime a transmission-type crystal spectrometer was developed based on the DuMond design [94]. The choice for a transmission-type spectrometer was encouraged by the larger solid angle achievable with the same crystal geometry due to the perpendicular orientation of the lattice planes with respect to the crystal surface, as seen in Figure 5.8. Additionally, the efficiency of the diffraction for small Bragg angles ${ }^{28}$ is supposedly higher in transmission than in reflection [99].

The DuMond geometry, which employs a cylindrically bent crystal, requires that the radius of curvature of the crystal is the diameter of the so-called Rowland circle and that the center of the source (or spectrometer entrance slit) and the center of the crystal are part of this circle. A sketch of the geometric requirements is shown in Figure 5.9. A crystal that is curved in such a way offers the incoming $x$ rays a much larger area with the same Bragg angle in comparison with a flat crystal. Thus, the curved design increases the spectral throughput, i.e., the transmitted flux per energy interval, tremendously. Correct curvature and placement also strongly reduce the overall bandwidth of the diffracted $x$ rays. The goal of the SuperEBIT transmission spectrometer design was to reduce this bandwidth such that the spectrometer can be used as a monochromator, where no spatially resolved detection of the diffracted $\mathrm{x}$ rays is necessary and detectors with almost $100 \%$ counting efficiency can be used, like highpurity germanium detectors. The energy dispersion of the germanium detector in such an arrangement serves as a tool for the suppression of the background radiation. The efficiency and the achievable resolving power of this transmission-type spectrometer are important parameters with respect to the feasibility of this instrument.

\footnotetext{
${ }^{28}$ For the transmitted diffracted $x$ rays the angle between the incoming $x$ ravs and the lattice plane of interest is also referred to as "Laue angle" instead of Bragg angle.
} 
(a)
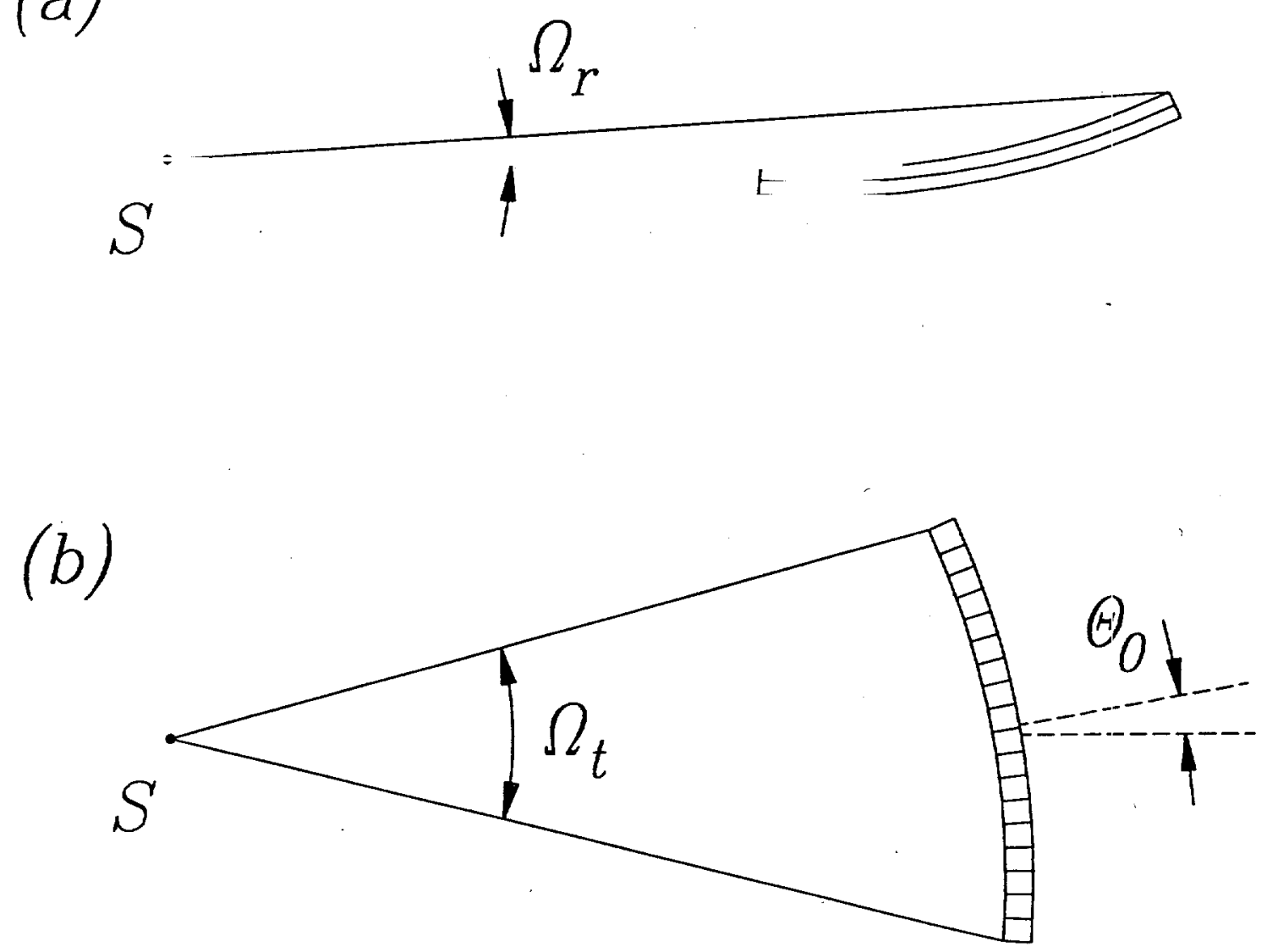

Figure 5.8: For the same crystal geometry and small Bragg angles, i.e., high x-ray energies, the solid angle $\Omega_{r}$ accessible using a reflection-type crystal is much smaller than the solid angle available in the transmission-type geometry, $\Omega_{t}$. The ratio of the solid angles $\left(\Omega_{t} / \Omega_{r}\right.$ is proportional to the cotangent of the Bragg angle of the center beam $\theta_{0}$.

\subsubsection{Resolving Power}

The resolving power, $\lambda / \Delta \lambda$ or $E / \Delta E$, is usually, inferred from the full width at half maximum of a recorded spectral line, of which it is known that the line broadening is dominated by the instrumental properties, i.e., by the apparatus profile. In designing the spectrometer, the resolving power was calculated from the bandwidth of the diffracted $x$ rays, or, more specifically, the part of the diffracted $x$ rays that hits the 


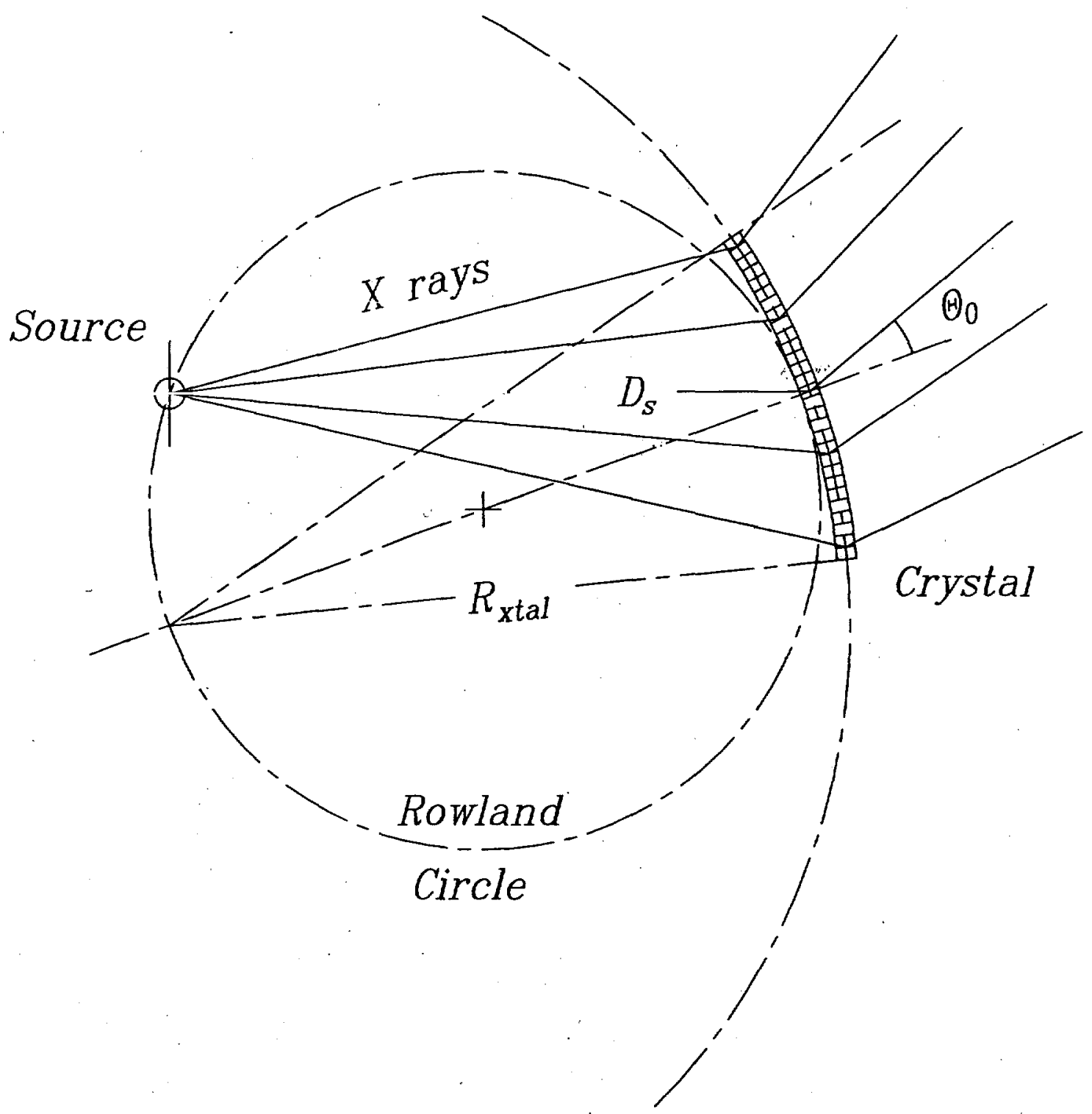

Figure 5.9: Geometric requirements of the DuMond-type crystal spectrometer. The curvature of the cylindrically bent crystal $R_{x t a l}$ equals the diameter of the Rowland circle. Placing the center of the source and the center of the crystal on the Rowland circle defines the distance between these points as a function of the central Bragg angle $\theta_{0}$, in particular, $D_{s}=R_{x t a l} \cos \theta_{0}$.

detector, weighted by the expected spectral intensity. The calculations are based purely on the geometric quantities of the instrument and the source, and the $\mathrm{x}$ rays 
were traced with the tools of geometrical optics only. The result is presented in two ways. First, as a contour plot showing the isoenergetic lines in the detector plane, i.e., the lines along which the incoming $\mathrm{x}$ rays have the same energy. The energy values listed in these plots are the energy differences with respect to the energy of interest. And second, as a line profile graph, which is obtained with a finite-element method. The profile is based on a simple numerical model where the source is divided in equal volume elements and it is assumed that the emitted flux is the same for all volume elements. For each of these finite elements the energy distribution of the $\mathrm{x}$ rays on the detector plane is calculated. Sorting the calculated values according to their energy values allows a concise presentation in form of a histogram containing the number of "counts", i.e., calculated values, within a predefined x-ray energy interval. The line width of this calculated intensity profile is an appropriate measure for the instrumental resolution.

The calculations were customized for estimating the resolving power of the transmissiontype crystal spectrometer with respect to the high-resolution measurements of the $\mathrm{K} \alpha$ emission of heliumlike and hydrogenlike xenon of interest to the present work. Thus, the $30630-\mathrm{eV} 1 s 2 p^{1} P_{1} \rightarrow 1 s^{2}{ }^{1} S_{0}$ transition in heliumlike xenon was taken as the $\mathrm{x}$-ray energy of interest ${ }^{29}$. Two different crystal curvatures were considered, utilizing in both cases a quartz crystal with a (13̄0) orientation, cut for transmission-type applications. The $2 \mathrm{~d}$ spacing of this crystal is approximately $2.36 \AA[101]$ and, thus, the Bragg angle for the diffraction of the $30-\mathrm{keV} \times$ rays is around $10^{\circ}$. The calculations also account for two different detector sizes, a $16-\mathrm{mm}$ diameter $\left(200 \mathrm{~mm}^{2}\right)$ and a $36-\mathrm{mm}$ diameter $\left(1020 \mathrm{~mm}^{2}\right)$ germanium detector, respectively. For the sake of simplicity the two sets of calculations will in following be referred to as SE28 and SE37. An overview of the various geometric quantities is given in Table 5.3 and graphically in Figure 5.10.

The geometrical ray tracing methods were also applied for determining the impact

\footnotetext{
${ }^{29}$ This theoretically predicted value for the transition energy was taken from Cheng et al. [100].
} 
Table 5.3: Geometric quantities necessary for the estimation of the resolving power of a transmission-type spectrometer setup. Two different setups are considered for the calculations, named $S E 28$ and SE37. For both measurements a quartz $(13 \overline{4} 0)$ crystal was used which has a 2 -d spacing of $2.3604 \AA[101]$. The $x$-ray energy of interest is $30630.36 \mathrm{eV}$, which is the $1 s 2 p^{1} P_{1} \rightarrow 1 s^{2}{ }^{1} S_{0}$ transition energy in heliumlike xenon according to Cheng et al. [100]. The Bragg angle, thus, equals $9.8741^{\circ}$. $R_{x t a l}$ is the radius of curvature of the crystal, $D_{s}$ the distance between the crystal and the electron beam, $D_{d}$ the distance between the crystal and the detector, and $A_{\text {eff }}$ the detector area. A cylinder of $12.7 \mathrm{~mm}$ height and $90 \mu \mathrm{m}$ diameter represents the $x$-ray source, i.e., the spectroscopically accessible area of the SuperEBIT trap region.

\begin{tabular}{rcc}
\hline \hline Quantity & SE28 & SE37 \\
\hline$R_{\text {xtal }}(\mathrm{mm})$ & 2713.8 & 2511.4 \\
$D_{s}(\mathrm{~mm})$ & 2673.6 & 2474.2 \\
$D_{d}(\mathrm{~mm})$ & 130 & 215 \\
$A_{\text {eff }}\left(\mathrm{mm}^{2}\right)$ & 201 & 1018 \\
\hline
\end{tabular}

of misaligned components onto the resolving power. Such a simulation is extremely important since it determines which instrumental features are the limiting factors in the spectrometers performance and, thus, have to receive utmost attention during the adjustment procedure. The variable parameters implemented into the $\mathrm{x}$-ray tracing model are:

- orientation of the crystal and of the lattice planes with respect to the crystal surface;

- size: position, and orientation of the $\mathrm{x}$-ray source;

- size, position, and orientation of the detector; 


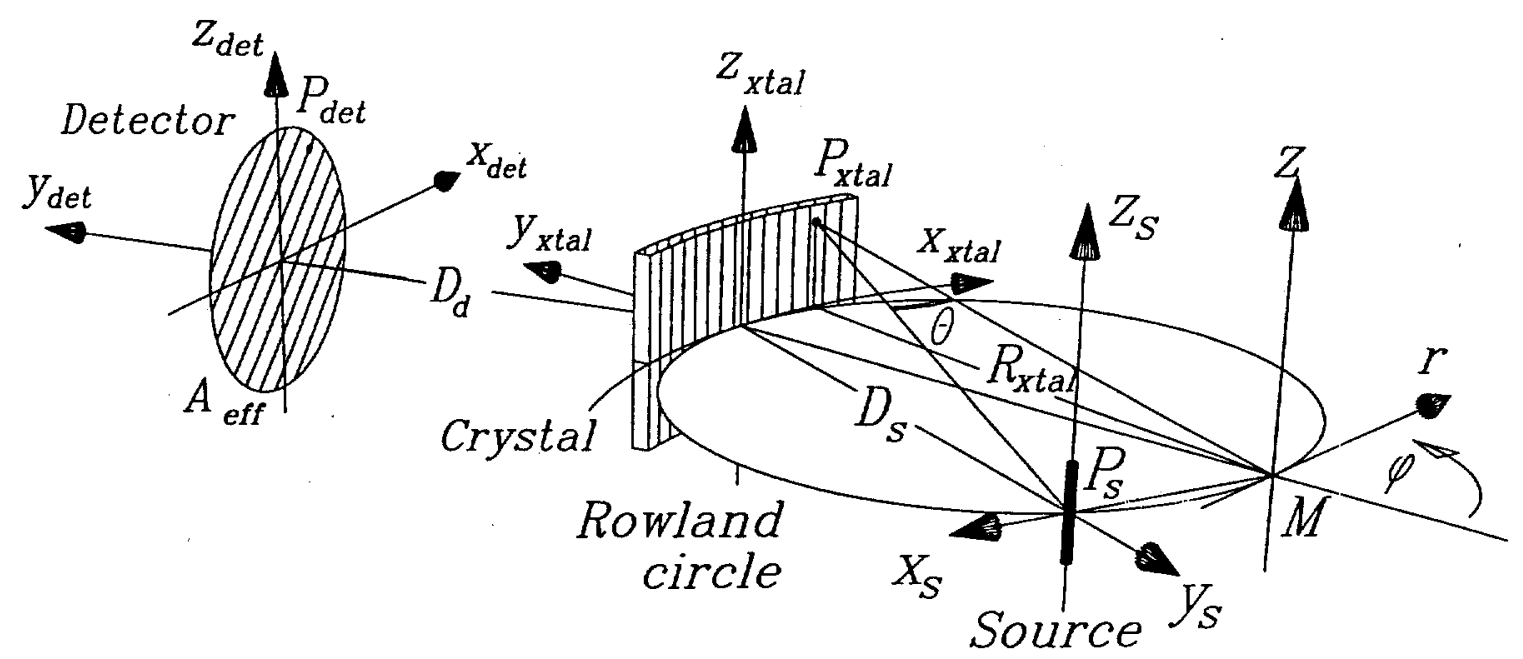

Figure 5.10: Sketch of the geometric components important for the calculation of the transmission spectrometer's resolving power. A cyindrical coordinate system $(r, \varphi, z)$ centered at point $M$ describes the curvature of the crystal $R_{x t a l}$. The Bragg angle $\theta$ is defined by the triangle $\left(M, P_{s}, P_{x t a l}\right)$, where $P_{s}$ is the point of $\mathrm{x}$-ray emission and $P_{x t a l}$ the location of $\mathrm{x}$-ray impact on the crystal. The energy of the diffracted $\mathrm{x}$ rays at the point of intersection $P_{\text {det }}$ with the detector area $A_{\text {eff }}$ was determined as a function of the distance $D_{s}$, the location of the x-ray emitting point $P_{s}$ with respect to the coordinates $\left(x_{s}, y_{s}, z_{s}\right)$, and the crystal orientation with respect to the $x_{x t a l}$ (tilt), $y_{x t a l}$ (twist), and $z_{x t a l}$ (turn) axis. These calculations allow the estimation of the sensitivity of this arrangement with respect to experimental uncertainties in the adjustment of the components.

Each component has its own coordinate system and, thus, the motions tilt, twist, and turn are defined as rotational motions relative to the $\mathrm{x}, \mathrm{y}$, and $\mathrm{z}$ axis of the component's coordinate system, respectively.

Starting with the assumption of perfect alignment and choosing the origin of the source coordinate system, which in this case is part of the Rowland circle, as the location of the emitting $\mathrm{x}$ rays yields an incredibly small band width. In particular, the difference between the $\mathrm{x}$-ray photons with the highest and the lowest energy that hit the detector is only $0.12 \mathrm{eV}$ for the SE28 setup, and $0.7 \mathrm{eV}$ for the SE37 setup utilizing a five times larger detector area. The energy distribution across the detector plane is shown as a contour plot in Figure 5.11(a) and Figure 5.12(a) for the SE28 
and SE37 setup, respectively. The actual detector area is marked by a circle in the plots "(f)".

The effect of a variation in the distance between the source and the crystal is shown in the plots (b) and (c) of those Figures. Assuming some arbitrary initial uncertainty in this distance $D_{s}$ of a couple of millimeters the source was "moved" by five millimeters in the $y_{s}$ direction (see Figure 5.10). For the SE28 the energy distribution in (b) is based on a distance of $D_{s}=2670 \mathrm{~mm}$, i.e., $3.6 \mathrm{~mm}$ inside the Rowland circle. Since the misalignment is restricted to the horizontal plane it affects mainly the energy distribution in horizontal direction which now exhibits a much stronger gradient and an overall energy difference of $1.3 \mathrm{eV}$. Moving outside the Rowland circle has an equivalent effect. Plot (c) is the result assuming a distance of $D_{s}=2680 \mathrm{~mm}$ which is $6.4 \mathrm{~mm}$ from the ideal location. Again the energy band width is dominated by the gradient in horizontal direction. As expected, the sign of the deviation from the energy of interest changes when crossing the Rowland circle. The maximum difference between the $\mathrm{x}$-ray photons which hit the detector is $2.3 \mathrm{eV}$. The Figures 5.12(b) and (c) display the equivalent scenario for the SE37 geometry. Ideally, the distance between the crystal and the source should be $D_{s}=2474.2 \mathrm{~mm}$, as it is the case for the energy distribution calculation in (a). Decreasing the distance by $4.2 \mathrm{~mm}$ yields the distribution plotted in (b), which shows a maximum energy difference of $3.8 \mathrm{eV}$ between the left and the right edge of the detector area. Moving the source $5.8 \mathrm{~mm}$ outside the Rowland circle increases this energy difference to $5.6 \mathrm{eV}$, as shown in (c).

Another test of the sensitivity of the spectrometer setup with respect to uncertainties in the adjustment, assumes a one-degree tilt of the crystal, i.e., a rotation around the crystal's $x$ axis $\left(x_{x t a l}\right)$ of $1^{\circ}$ such, that the top part of the crystal points away from the source, the bottom part, thus, is slightly closer to the source. The source-to-crystal distance is assumed to be at $D_{s}=2675 \mathrm{~mm}$ for the SE2 8 setup and at $D_{s}=2475 \mathrm{~mm}$ in SE3\%. This type of misalignment causes a dominant deviation in 

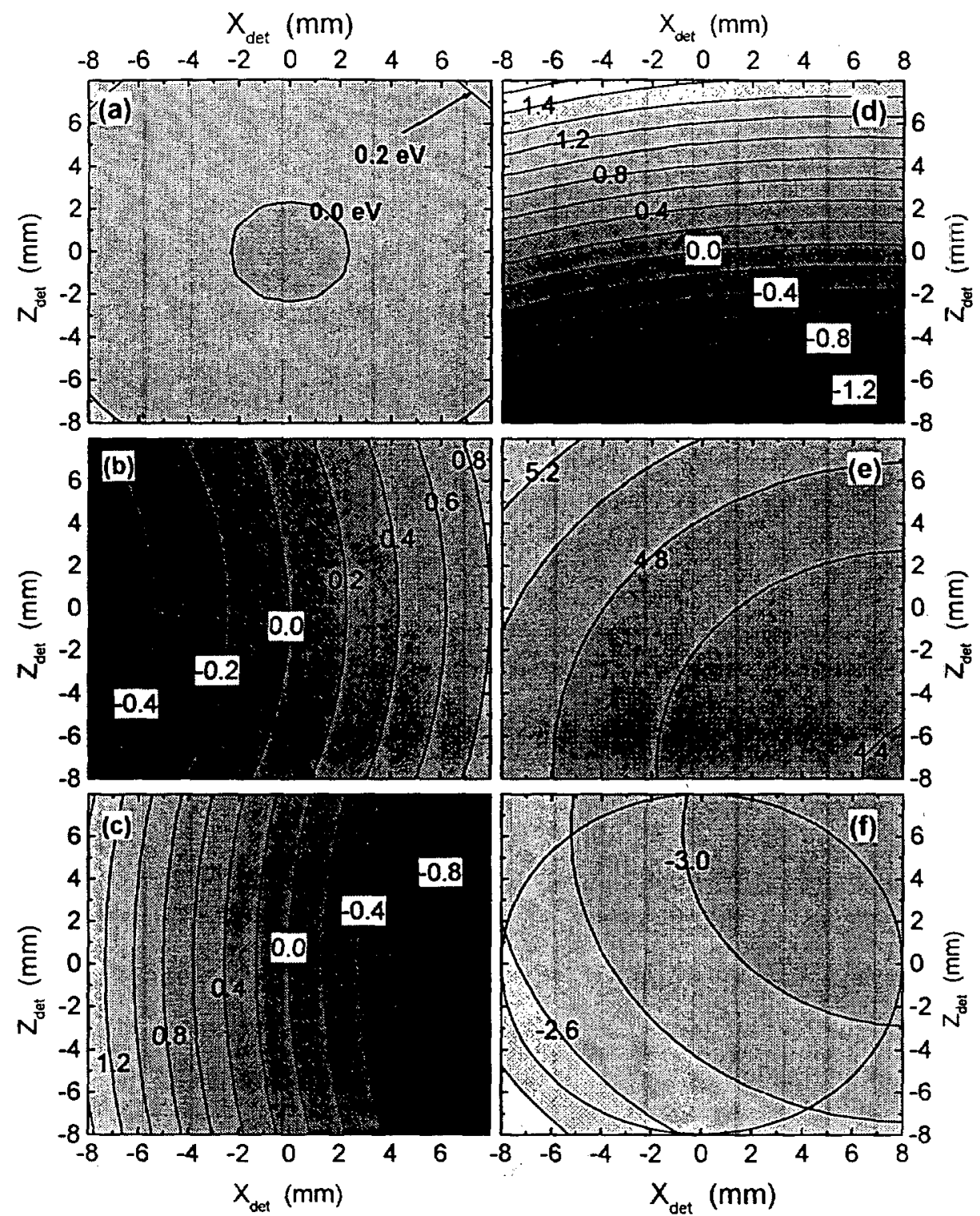

Figure 5.11: Distribution of the deviation from the $30630-\mathrm{eV}$-ray energy of interest for the diffracted $\mathrm{x}$ rays across the detector area which is $16 \mathrm{~mm}$ in diameter, see the circle in plot (f). These calculations are based on the geometry denoted SE28 in Table 5.3. Assuming perfect alignment yields the distribution shown in (a) $\left(D_{s}=2673.6 \mathrm{~mm}\right)$. The contour plots (b) and (c) represent the distributions when the source is not part of the Rowland circle $\left(D_{s}=2670 \mathrm{~mm}\right.$ and $2680 \mathrm{~mm}$, respectively.). The effect of tilting and twisting the crystal by $1^{\circ}$ is shown in (d) and (e). All but the calculation presented in (f) consider the center of the source as the location of $x$-ray enission. "Placing" the point of emission to the top edge of the source volume yields the energy distribution shown in plot (f). 


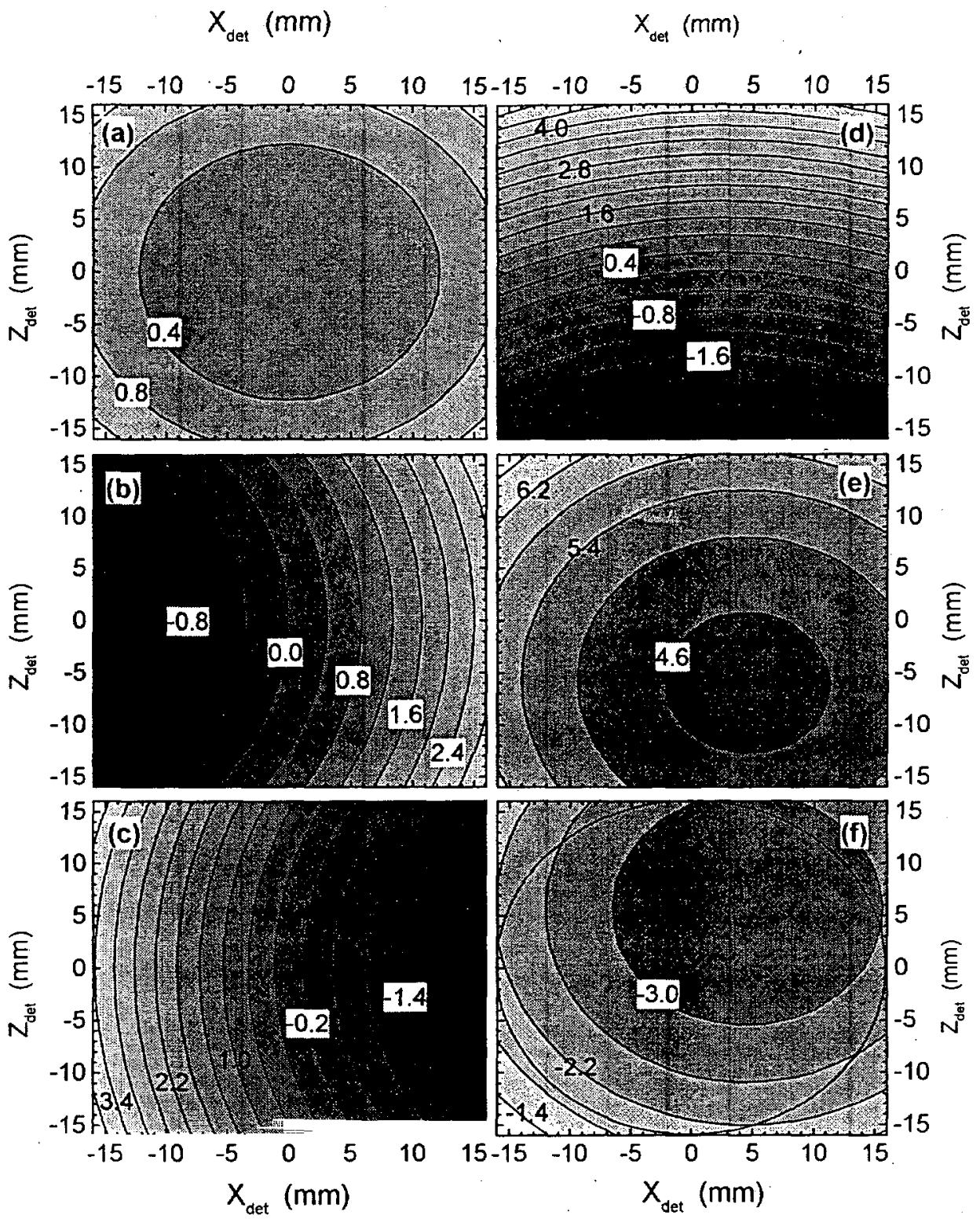

Figure 5.12: Distribution of the deviation from the $30630-\mathrm{eV}$-ray energy of interest for the diffracted $\mathrm{x}$ rays across the detector area which is marked by the circle in plot (f). These calculations are based on the geometry denoted SE37 in Table 5.3. Assuming perfect alignment yields the distribution shown in (a) $\left(D_{s}=2474.2 \mathrm{~mm}\right)$. The contour plots (b) and (c) represent the distributions when the source is not part of the Rowland circle $\left(D_{s}=2470 \mathrm{~mm}\right.$ and $2480 \mathrm{~mm}$, respectively.). The effect of tilting and twisting the crystal by $1^{\circ}$ is shown in (d) and (e). All but the calculation presented in $(\mathrm{f})$ consider the center of the source as the location of $\mathrm{x}$-ray emission. "Placing" the point of emission to the top edge of the source volume yields the energy distribution shown in plot (f). 
the vertical direction as seen in Figure 5.11(d) and Figure 5.12(d). The comparably smooth gradient in horizontal direction is due to the "non-perfect" $D_{s}$ values. Surprisingly, this rather small tilt shows so far the strongest impact with respect to the energy spread of the counted $\mathrm{x}$ rays, in particular, $3.0 \mathrm{eV}$ across the small and $7.0 \mathrm{eV}$ across the large detector area. If the lattice planes are twisted, i.e., rotation about the $y_{x t a l}$-axis, the Bragg angle changes collectively for each point of the crystal. Thus, even if there is a big offset with respect to the ideal setup, the difference between the highest and lowest $\mathrm{x}$-ray energy of the detected photons is relative small. The distribution shown in the contour plots (e) are based on a one-degree twist but no tilt. The band width for this case is about $0.6 \mathrm{eV}$ for $S E 28$ and $1.6 \mathrm{eV}$ for $S E 37$. From Figure 5.10 it is obvious that tilting, twisting, or turning the detector only decreases the effective detector area by the cosine of the engaged angle. A smaller effective detector area increases the resolving power but decreases the overall efficiency of the spectrometer.

So far, all calculations considered only the center of the source as the point of $x$-ray emission. The source geometry encountered at SuperEBIT, however, is approximately a $12.7-\mathrm{mm}$ high cylinder with a diameter of roughly $90 \mu \mathrm{m}$. Placing the source point at the top "left" edge of the cylinder, i.e., at $x_{s}=45 \mu \mathrm{m}$ and $z_{s}=6.3 \mathrm{~mm}$. The result is shown in Figure 5.11(f) and Figure 5.12(f). Choosing a source point on the opposite side of the $\left(y_{s}, z_{s}\right)$ plane, i.e., at $x_{s}=-45 \mu \mathrm{m}$ yields qualitatively the same result except of the inverted signs. The energy difference between the detected $x$-rays due to the spatial extension of the source is approximately $6.1 \mathrm{eV}$ for the $S E 28$ geometry and about $6.4 \mathrm{eV}$ for $S E 37$.

The energy difference values, however, are not representative for the actual distribution of the $\mathrm{x}$-ray energy values. The determination of the resolving power, thus, requires sorting of the "detected" photons according to their energy. In Figure 5.13(a) the result of sorting one of the contour plot data fields of the SE37 setup is given in form of a histogram showing the number of counts with respect to the energy inter- 
val. This intensity plot only accounts for one single source point. Accumulating the sorted values for all different source point positions gives the intensity profile shown in Figure 5.13(b). The setup for this calculation includes a $0.5^{\circ}$ tilt of the crystal and a 5-mm misalignment with respect to the distance $D_{s}$. The width of this calculated intensity profile is only about $3 \mathrm{eV}$ for the prominent peak and about $6 \mathrm{eV}$ including the wider bottom of this structure, which is significantly less than the $10 \mathrm{eV}$ difference between the lowest and the highest $\mathrm{x}$-ray energy counted in this simulation. Taking conservatively the $6 \mathrm{eV}$ resolution the resolving power would be in the order of 5000. This estimate, however, is based on the assumption that the curvature of the crystal is perfectly cylindrical. An indirect measurement revealing the quality of the crystal curvature by imaging a $25-\mu \mathrm{m}$ point source using visible light with the curved quartz crystal lead to a measured image width of $230 \mu \mathrm{m}$ for the crystal which is implemented in the SE37 setup. For the image width for the crystal used in the other set, unfortunately, only an upper limit is available, which is $400 \mu \mathrm{m}$. The broadening due to the non-perfect curvature of the crystal has a similar impact as the width of the source. For the SE37 setup, for example, the band width of the detected photons is roughly $16 \mathrm{eV}$, i.e., about 2.5 times larger than the broadening caused by the width of the x-ray source. The resolving power for the SE37 geometry is, thus, reduced to 2000. Still about 20 times higher than the resolving power of the best germanium detectors for $30-\mathrm{keV}$ x-ray radiation.

The SE37 geometry was tested using a $1-\mathrm{mCi}{ }^{133} \mathrm{Ba}$ source and a $75-\mu \mathrm{m}$ wide and 12.7-mm tall slit made out of $2.4-\mathrm{mm}$ thick tantalum. The radioisotope ${ }^{133} \mathrm{Ba}$ decays by capturing an innershell electron, thus, creating an innershell excited cesium atom. The characteristic K-shell $\mathrm{x}$ rays of cesium are centered around $30974 \mathrm{eV}$ $\left(\mathrm{K} \alpha_{1}\right)$ and $30626 \mathrm{eV}\left(\mathrm{K} \alpha_{2}\right)$. The spectrum was obtained by rotating the crystal in small increments and counting the number of $30-\mathrm{keV} x$ rays which hit the germanium detector for a certain time before rotating the crystal to the next position. A detailed discussion about the rotating mechanism is given in Section 7.1.4 on page 169. Fig- 


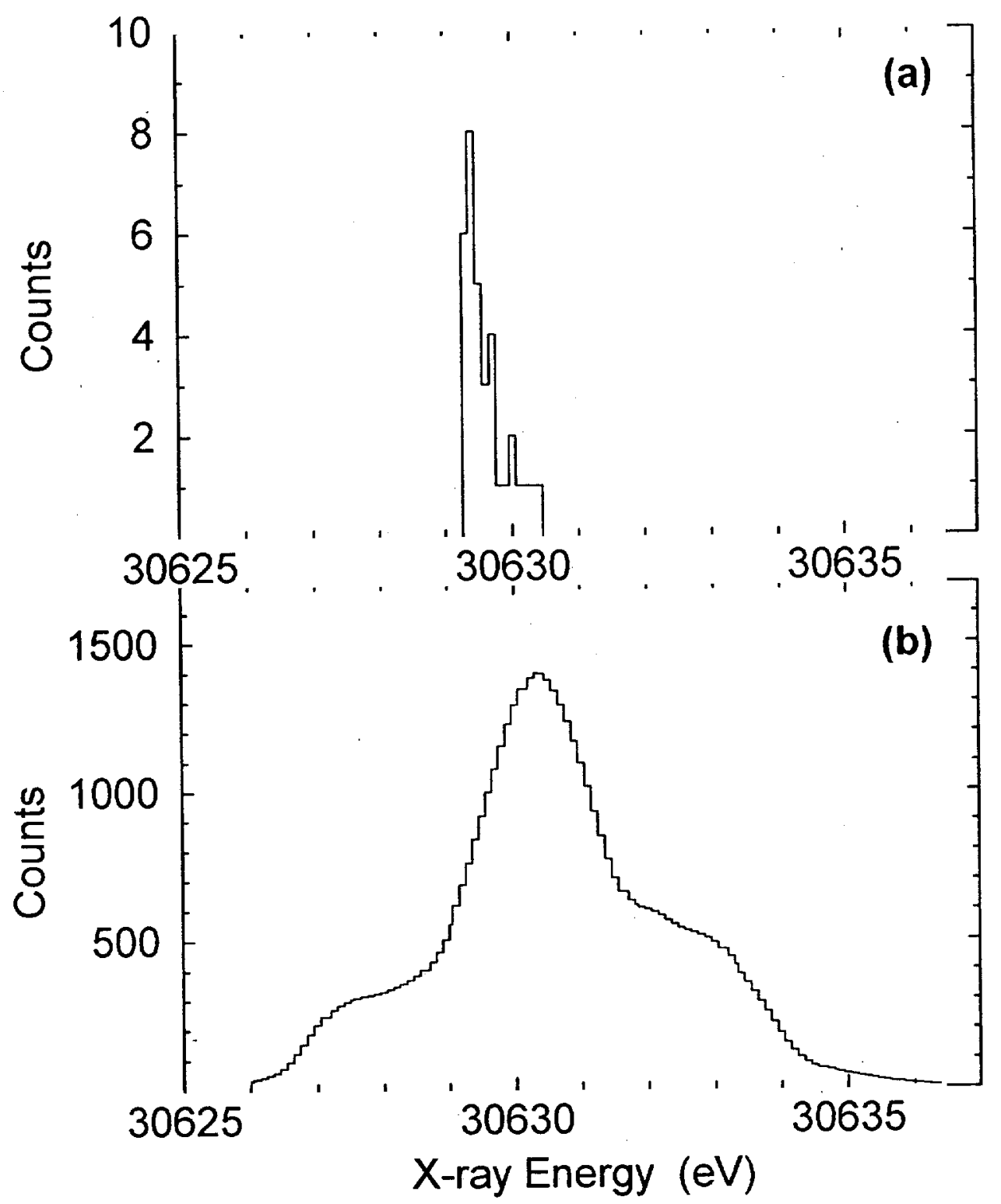

Figure 5.13: Simulation of the transmission-type crystal spectrometer apparatus profile. Sorting the number of diffracted $\mathrm{x}$ rays which hit the detector according to their energy yields a histogram which represents the instrumental resporise with respect to the intensity profile. (a) is the narrow distribution obtained when considering a point source only. Accounting for the spatial extent of the source, in particular, the electron beam trap geometry in the SuperEBIT trap region, gives the much wider distribution shown in (b). The setup for this calculation includes a $0.5^{\circ}$ tilt of the crystal and a $5-\mathrm{mm}$ misalignment with respect to the distance $D_{s}$ (see Figure 5.10). 
ure 5.14 shows the result of several scans over both lines. The measured line width of $30 \mathrm{eV}$ contains the instrumental width and also the natural line width of the cesium $\mathrm{K} \alpha$ radiation which is about $15 \mathrm{eV}$ |102]. Deconvolution of the measured profile using the $15-\mathrm{eV}$ broad Lorentzian component leads to an instrumental line width of about $25 \mathrm{eV}$, which is equivalent to a resolving power of a little bit more than 1200 , therefore, successfully demonstrating the feasibility of this transmission-type crystal spectrometer design.

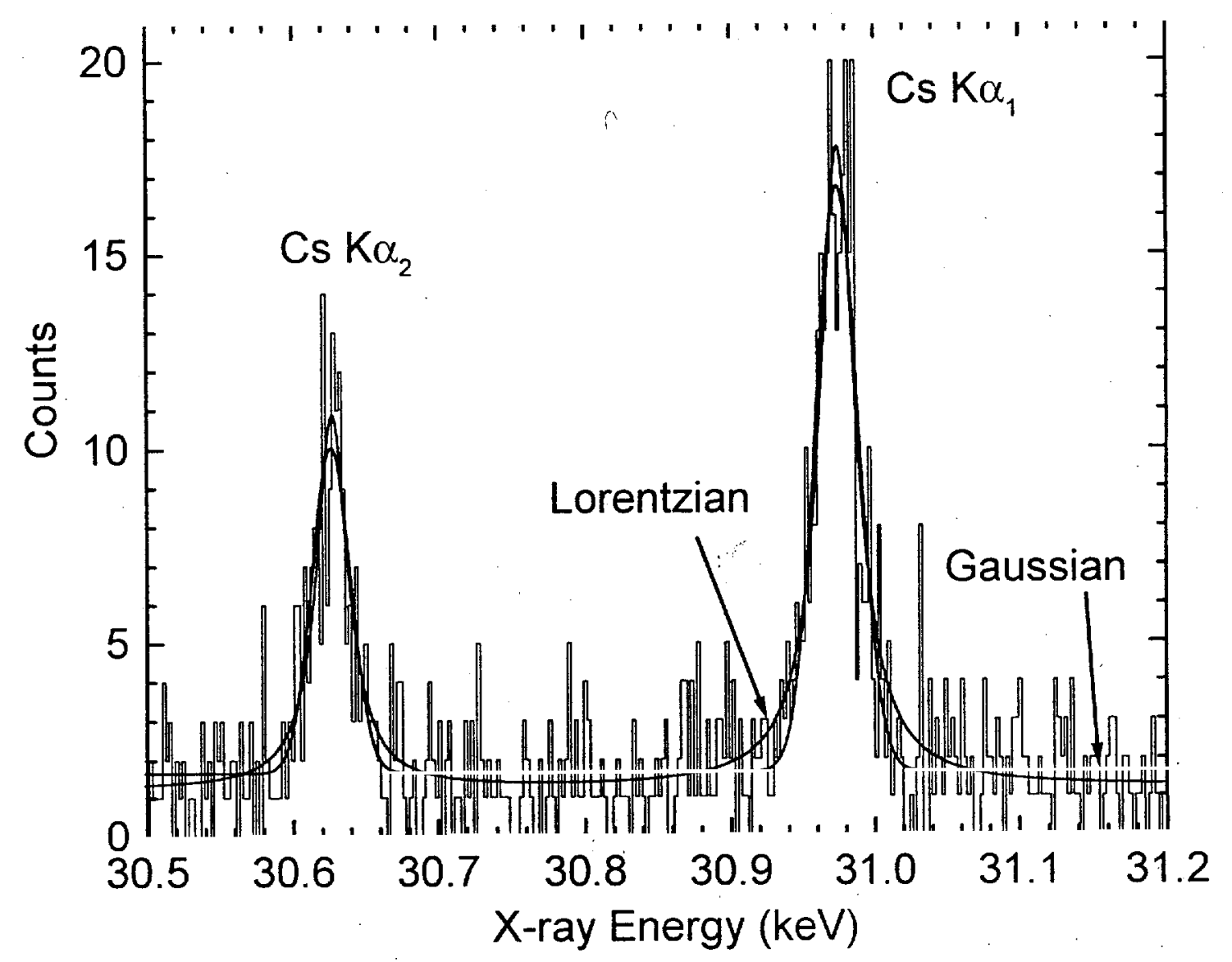

Figure 5.14: Highly resolved spectrum of the cesium $K \alpha$ radiation demonstrating the feasibility of the transmission-type spectrometer designed for high-resolution hard $\mathrm{x}$-ray measurements at SuperEBIT. The transition energies of the cesium lines are $30626.3 \mathrm{eV}$ for the smaller $\mathrm{Cs} \mathrm{K} \alpha_{2}$ transition and $30974.1 \mathrm{eV}$ for the larger Cs $\mathrm{K} \alpha_{1}$ transition (see also Table 7.19, page 183). 


\section{Spectroscopy on heliumlike krypton, $\mathrm{Kr}^{34+}$}

Recent measurements on the Test Fusion Tokamak Reactor (TFTR) at the Princeton Plasma Physics Laboratory [103] and on the OMEGA Laser system at the Laboratory for Laser Energetics in Rochester |104| have demonstrated the feasibility of using highly charged krypton for spectroscopy based plasma diagnostic. Krypton appears to be the element of choice because its admixture in trace amounts can be precisely controlled as it was shown at the TFTR experiment. Moreover, it has been already suggested for use in radiative cooling of the plasma edge region and, thus, for reducing the heat load at the diverter [105]. In a comparison with low- $\mathrm{Z}$ candidates for cooling purposes, like carbon or neon, higher $\mathrm{Z}$ elements are more efficient. Therefore, a much smaller quantity of higher $-Z$ material is needed for sufficient cooling than when implementing low $-Z$ materials. In fact, it appears that the effective $Z$ of the tokamak core plasma can be kept lower when admixing a high $-\mathrm{Z}$ versus a low $-\mathrm{Z}$ coolant while achieving the same amount of radiative cooling.

Successful implementation of x-ray spectroscopy as a precise tool for plasma diagnostics strongly depends on the availability of a comprehensive, reliable atomic physics database that includes transition energies as well as excitation, recombination, and ionization cross sections [106]. The following measurement performed at EBIT contributes to this database. Applying high-resolution crystal spectroscopy tools, the energies of the $\mathrm{n}=2 \rightarrow 1$ transitions in heliumlike krypton, $\mathrm{Kr}^{34+}$, and in innershell excited lithiumlike and berylliumlike krypton, $\mathrm{Kr}^{33+}$ and $\mathrm{Kr}^{32+}$, respectively, were determined relative to previously measured transitions in heliumlike iron and well known theoretical values of the transition energies in hydrogenlike manganese. The first part of this Chapter focuses on the direct excited (DE) transitions and discusses the inter-order calibration of the heliumlike krypton transition energies. The measurement presented in the second part is centered around the dielectronic recombination (DR) lines in highly charged krypton ions, in particular, the separation and identification of the so called KLL resonances. 


\subsection{Measurements of the $\mathbf{n}=2 \rightarrow 1$ transition energies of heliumlike krypton}

Besides their application with respect to plasma diagnostics, spectra of heliumlike ions are also a subject of intense research interest from the atomic physics point of view as mentioned in Chapter 3. Especially high-Z heliumlike ions, as differences among predictions tend to increase strongly with atomic number. In the case of $\mathrm{Kr}^{34+}$, for example, which was until recently, i.e., until the measurements on highly charged xenon presented in Chapter 7 , the heaviest heliumlike ion that was investigated by means of high-resolution crystal spectroscopy, a significant disagreement was found between the measured and theoretically predicted values for the $\mathrm{n}=2 \rightarrow 1$ transition energies [107]. In particular, the measurement in Ref. [107] (by Indelicato et al.) included the $1 s 2 p^{1} P_{1} \rightarrow 1 s^{2}{ }^{1} S_{0}$, and $1 s 2 p^{3} P_{1} \rightarrow 1 s^{21} S_{0}$ transitions in heliumlike krypton. Their result of $13115.45(30) \mathrm{eV}$ and $13026.8(3) \mathrm{eV}$ is by $0.75 \mathrm{eV}$ and $0.48 \mathrm{eV}$ separated from the closest currently available theoretically predicted value. Additionally, the various theoretical values themselves exhibit differences in the same order of magnitude. Thus, there is a strong interest in remeasuring the energies of the heliumlike krypton $\mathrm{K} \alpha$ transitions.

\subsubsection{Experimental setup}

The wavelengths and, thus, the energies of the $1 s 2 p^{1} P_{1} \rightarrow 1 s^{21} S_{0}, 1 s 2 p^{3} P_{2} \rightarrow 1 s^{21} S_{0}$, $1 s 2 p^{3} P_{1} \rightarrow 1 s^{21} S_{0}$, and $1 s 2 s^{3} S_{1} \rightarrow 1 s^{21} S_{0}$ transitions in heliumlike krypton $\left(\mathrm{Kr}^{34+}\right)$ have been measured utilizing a high-resolution von-Hámos-type crystal spectrometer (see Section 5.2.2) optimized for operation at the EBIT facility. The spectrometer was equipped with a cylindrically bent (200)-LiF crystal with a radius of curvature of $75 \mathrm{~cm}$. The $2 \mathrm{~d}$ spacing of the (200) oriented lithium fluoride crystal equals $4.027 \AA|108|$ or $4.028 \AA|109|$. The difference of $0.001 \AA$ is used as a measure for the uncertainty in the $2 \mathrm{~d}$ spacing. The impact of the uncertainty in the $2 \mathrm{~d}$ spacing on the measured wavelengths is insignificant, since the measurement is based on an "inter- 
nal" calibration using spectral lines of known wavelength and, thus, does not depend on the absolute value of the $2 \mathrm{~d}$ spacing. However, a "good" value for the $2 \mathrm{~d}$ spacing is very beneficial to the accurate adjustment of the setup. The implementation of the von Hámos spectrometer geometry at the EBIT facility is shown in Figure 6.1. The spectrometer is set to a nominal Bragg angle $\theta=28^{\circ}$. This setup measures

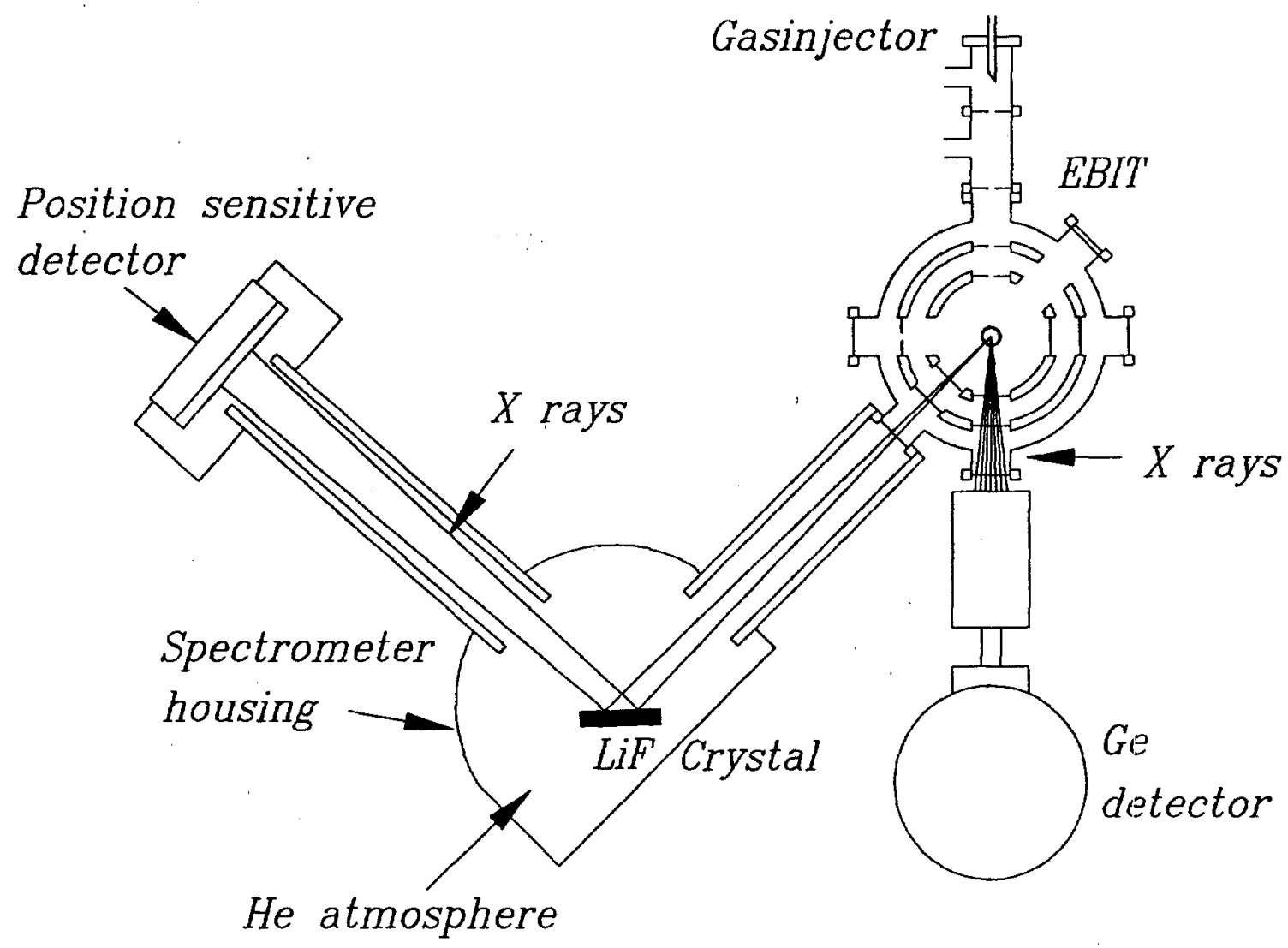

Figure 6.1: A sketch of the EBIT device is shown in a cross section with the electron beam in the center of the device and perpendicular to the page. The main components of the von Hámos spectrometer are the crystal, the position sensitive detector, and the spectrometer housing. The housing is necessary for applying a helium gas atmosphere which tremendously reduces the absorption of the low-energy $\mathrm{x}$ rays. The gas injector was used for the introduction of neutral krypton into the trap region. The germanium detector serves only as a charge balance monitor in this particular measurement.

the krypton $\mathrm{K} \alpha$ radiation in second order and the $\mathrm{Ly}-\alpha_{1,2}$ lines of manganese and 
the transitions $1 s 2 s^{3} S_{1} \rightarrow 1 s^{21} S_{0}$ and $1 s 2 s^{2} 2 p^{1} P_{1} \rightarrow 1 s^{2} 2 s^{2}{ }^{1} S_{0}$ in heliumlilke and berylliumlike iron, used for calibration, in first order Bragg reflection. The position sensitive proportional counter was optimized for the energy range of the heliumlike krypton K-shell x rays (see Section 5.2.2.1, page 84). The operating parameters for the detector are: gas mixture $70 \% \mathrm{Xe}, 30 \% \mathrm{CH}_{4}$; gas pressure $5.38 \mathrm{bar}$ (78 psig) above $1 \mathrm{~atm}$; active volume $9.5 \times 3.0 \times 0.4 \mathrm{~cm}^{3}$; thickness of Be-window $1 \mathrm{~mm}$; applied voltage $4.2 \mathrm{kV}$. An efficiency of about $70 \%$ for first-order x-ray photons $(6.5 \mathrm{keV})$ and about $55 \%$ for the second-order krypton $\mathrm{K}$-shell $\mathrm{x}$ rays ( $13 \mathrm{keV}$ ) was achieved with these detector parameters. The efficiency values include the absorption due to the Be-windows in EBIT and the detector, respectively. The spatial resolution of the detector was between $80 \mu \mathrm{m}$ and $100 \mu \mathrm{m}$ over the whole detector area of interest.

For the observation of the krypton spectra neutral krýpton atoms are injected into the trap region using the continuous gas injector, for details see Section 4.2.1 (pg. 53). The gas injector pressure was in the $10^{-8}$-Torr range. Introduction of manganese and iron into the trap for the calibration of the recorded krypton spectra was accomplished with the MeVVA (see Section 4.2.2). In particular, a MeVVA was constructed with a cathode made from manganese and an iron trigger wire. In normal operation the MeVVA plasma contains mostly atoms and ions from the cathode material, which is manganese. Interchanging the electrical leads between cathode and trigger allows the injection of the trigger material, in this case iron. Therefore, the calibration measurements of iron and manganese $\mathrm{x}$ rays were made successively. Due to the independent methods of injection regarding krypton and one of the MeVVA materials, a krypton spectrum could be recorded simultaneously with one of the two calibration spectra. The ionization balance is optimized by choosing an appropriate electron beam energy, trap depth, cooling gas pressure, and the duration of the whole timing cycle and each of the components of the timing cycle, i.e., injection period (for pulsed mode), "cooking time" (ionization time necessary for reaching the high charge states), data acquisition time, and the time needed for dumping the "old" 
ions (see also Section 4.4, page 63 and, e.g., Ref. [57]).

\subsubsection{Resolving power of the von Hámos spectrometer}

The most important factors that limit the resolving power of the spectrometer are:

- the spatial resolution of the position sensitive proportional counter,

- the finite size of the source,

- the imaging properties of the von Hámos geometry, and

- the resolving power of the crystal.

Depending on the purpose of the spectroscopic investigation the thermal line broadening is or is not considered being part of the instrumental resolution. Measurements aiming for quantities linked to the atomic structure are definitely challenged by thermal line broadening and, thus, it is usually treated as an instrumental component. By contrast, spectroscopic investigations of plasmas would usually benefit from the fact that the resolution is "limited" by the thermal broadening. Focusing on the measurement of the transition energies, however, puts the thermal line broadening into the resolution-limiting category.

As mentioned above, the spatial resolution of the detector is between $80 \mu \mathrm{m}$ and $100 \mu \mathrm{m}$. The size of the source is given by the diameter of the electron beam which is roughly $60 \mu \mathrm{m}$ (see Section 4.1.3.1). The line spread due to the imaging properties is less than $30 \mu \mathrm{m}$, according to Figure 5.6. Thus, without accounting for the thermal broadening of the spectral lines, the resolving power of the setup would be $(E / \Delta E)_{\text {geom }}=14000$ to 16500 , where the index geom indicates that only geometrical quantities have been considered in this estimate. The difference to the measured resolution of $E / \Delta E \approx 2600$ for $\mathrm{Fe}^{24+}$ and $\mathrm{Mn}^{24+}$ in first, and $E / \Delta E \approx 4300$ for $\mathrm{Kr}^{34+}$ in second order shows that thermal line broadening and the resolving power of the crystal represent the main limitation regarding the resolving power. 
The operating conditions of EBIT are optimized towards the highest achievable x-ray flux. The deep axial trapping potential of $V_{a x i a l}=205 \mathrm{~V}$, and the high beam current, in particular $I_{\text {beam }}=180-200 \mathrm{~mA}$, however, create ion temperatures on the order of $700 \mathrm{eV}$, as shown in a set of measurements investigating the thermally broadened line profile in the emission spectra of heliumlike titanium ions $|110,111|$. The corresponding Doppler broadening, full width at half maximum (FWHM), is about $\Delta E_{T_{i o n}}=1.8 \mathrm{eV}$ for the heliumlike $\mathrm{Fe}^{24+}$ and the hydrogenlike $\mathrm{Mn}^{24+}$ lines, and about $\Delta E_{T_{i o n}}=2.9 \mathrm{eV}$ for the heliumlike $\mathrm{Kr}^{34+}$ lines. The line widths in the recorded spectra are $\Delta E_{\text {exp }}=(2.5 \pm 0.1) \mathrm{eV}$ for the hydrogenlike $\mathrm{Mn}^{24+}$ and the heliumlike $\mathrm{Fe}^{24+}$ lines and $\Delta E_{\text {exp }}=(3.05 \pm 0.05) \mathrm{eV}$ for the heliumlike $\mathrm{Kr}^{34+}$ lines. The width of the krypton lines is, thus, in good agreement with the expected ion temperature, which indicates that in second order Bragg reflection the resolving power is limited by the Doppler broadening, and the line profile is dominated by the thermal Doppler effect. In first order Bragg reflection, however, the main component limiting the resolving power appears to be the crystal. Therefore, the line profile in the first order spectra represents mainly the profile of the rocking curve of the crystal. The fact, that the spectral lines recorded in first and second order exhibit a different line profile requires the determination of the center of gravity for each spectral feature in order to successfully apply the inter-order calibration.

\subsubsection{Measurements}

The electron beam energy was selected far above the excitation threshold of the transition of interest and, thus, the recorded spectra contain only directly excited lines and lack the presence of satellite lines produced by dielectronic recombination. In particular, the electron beam energy was set to $19.5 \mathrm{keV}$ for the observation of the 13-keV Ka emission of highly charged krypton. For the manganese calibration the beam energy was set to about $12 \mathrm{keV}$, which yielded the highest countrate for the Ly- $\alpha$ emission. The iron data were collected at a $9-\mathrm{keV}$ electron beam energy. The 
energy thresholds for producing these ion species are $4.11 \mathrm{keV}$ to ionize lithiumlike $\mathrm{Kr}^{33+}, 8.14 \mathrm{keV}$ to ionize heliumlike $\mathrm{Mn}^{23+}$, and $2.05 \mathrm{keV}$ to ionize lithiumlike $\mathrm{Fe}^{23+}$.

The recorded spectra, i.e., the heliumlike krypton $\mathrm{K}$-shell spectrum together with the two calibration spectra featuring the manganese $L y-\alpha_{1,2}$ lines and the $1 s 2 s{ }^{3} S_{1} \rightarrow$ $1 s^{2} S_{0}$, labeled $z$, and $1 s 2 s^{2} 2 p^{1} P_{1} \rightarrow 1 s^{2} 2 s^{21} S_{0}$, labeled $\beta$, transitions in heliumlike and berylliumlike iron, are shown in Figure 6.2. The spectral lines of the helium-and lithiumlike ion :riecies are labeled according to the notation introduced by Gabriel |41|. Additionally, several collisional satellite lines of lower charge states are seen both in the spectrum of $\mathrm{Kr}$ and Fe. Some of the lithiumlike and berylliumlike satellites are labeled $(\mathrm{r}, \mathrm{q}, \mathrm{t}$, and $\beta$ ). The analyzed spectra represent data taken in about 44 hours, in particular, 9 hours of observation time was spent for manganese, 5 hours for iron, 26 hours for krypton, and 4 hours where krypton and iron were collected simultaneously. Taking the complete data set, shown in Figure 6.2, a countrate of about 100 counts per hour for the $1 s 2 p^{1} P_{1} \rightarrow 1 s^{2}{ }^{1} S_{0}$, and 45 counts per hour for the $1 s 2 s^{3} S_{1} \rightarrow 1 s^{2} S_{0}$ transition in $\mathrm{Kr}^{34+}$ can be derived. For the calibration lines the countrate was about 230 counts per hour for the Mn Ly- $\alpha_{1}$ line, and 315 counts per hour for the heliumlike iron transition, respectively.

The wavelengths and transition energy values of the calibration lines are listed in Table 6.1. Only the three lines, $\mathrm{Fe} z, \mathrm{Fe} \beta$, and $\mathrm{Mn} \mathrm{Ly}-\alpha_{1}$, are used for the calibration. These three lines determine both the absolute energy scale and the spectral dispersion. The remaining iron transitions $\mathrm{r}, \mathrm{q}$, and $\mathrm{y}$ have not been chosen for the calibration because there is no published energy or wavelength measurement of $r$ with sufficient accuracy (in plasma observations $\mathrm{r}$ blends with dielectronic satellite transitions), and because the lines $\mathrm{q}$ and $\mathrm{y}$ are too close to the edge of the illuminated area of the detector. Using the lines $\mathrm{q}$ and $\mathrm{y}$ as references would add an unknown systematic uncertainty to the determination of their line centroids. The use of the Mn Ly- $\alpha_{2}$ line for our calibration was abstained for the same reason. 

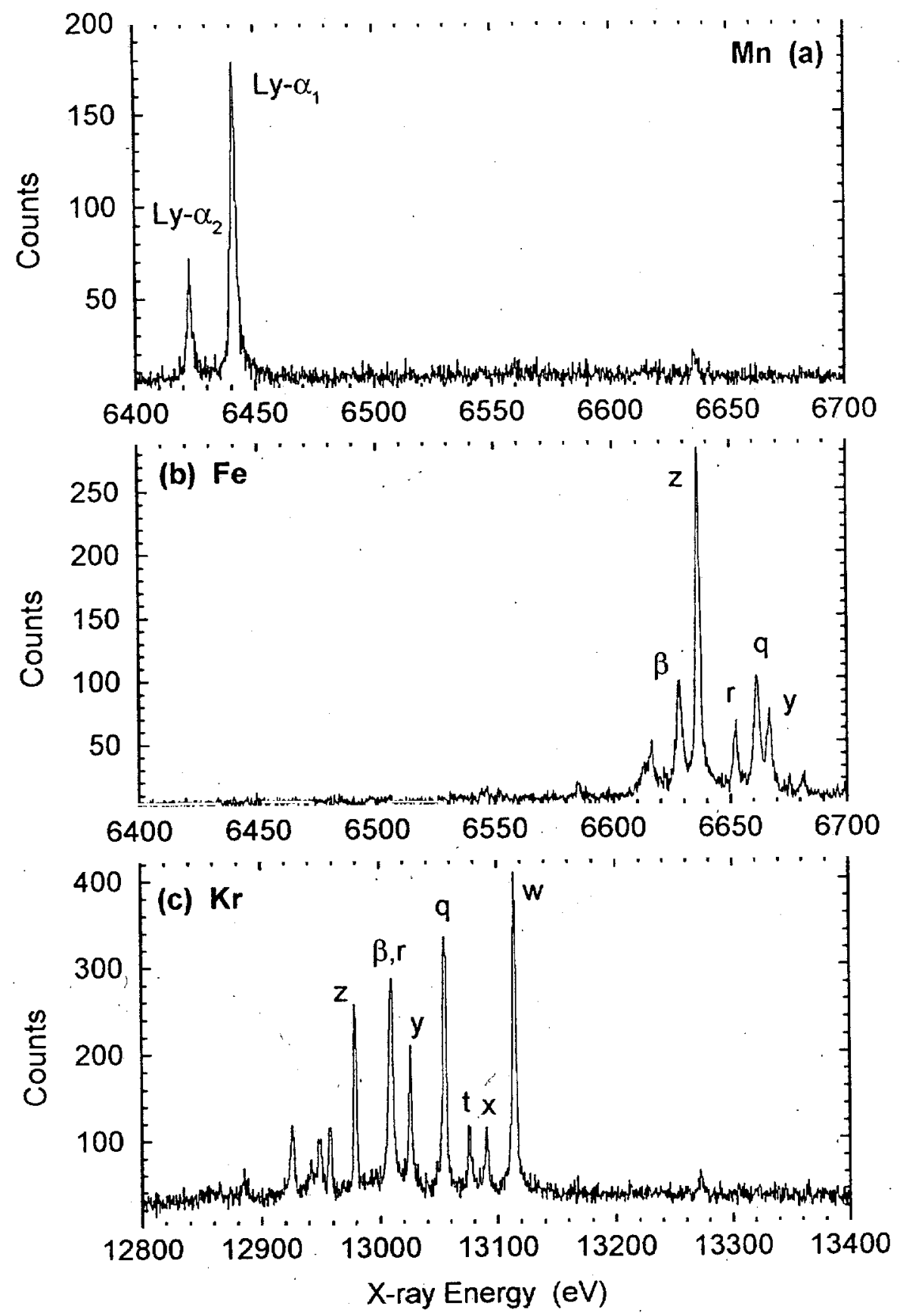

Figure 6.2: Calibration spectra displaying the hydrogenlike $\mathrm{Mn}^{24 \div} \mathrm{Ly}-\alpha_{1,2}$ lines (a) and of some lines of heliumlike $\mathrm{Fe}^{24+}(\mathrm{y}, \mathrm{z})$, lithiumlike $\mathrm{Fe}^{23+}(\mathrm{q}, \mathrm{r})$, and berylliumlike $\mathrm{Fe}^{22+}(\beta)$ (b). Both, the manganese and the iron spectra were taken in first order Bragg reflection. The Krypton K $\alpha$ spectrum (c) measured in second order shows the four heliumlike transitions ( $w, x, y, z)$, and some lithiumlike $(q, r, t)$ and berylliumlike ( $\beta$ ) krypton lines. 
Table 6.1: Wavelengths $\lambda$ and transition energies $E$ of the calibration lines. The values for the hydrogenlike manganese line, $\mathrm{Ly}-\alpha_{1}$, is taken from theoretical predictions by Mohr [112] and Johnson and Soff [27]. The values for the heliumlike and berylliumlike iron transitions, $\mathrm{z}$ and $\beta$, are measured values by Beiersdorfer et al. [113].

Transition

\begin{tabular}{cclc} 
& & \multicolumn{1}{c}{$\AA$} & $\mathrm{eV}$ \\
Mn Ly- $\alpha_{1}$ & $2 p_{3 / 2} \rightarrow 1 s_{1 / 2}$ & $1.9247230(8)$ & $6441.665(3)$ \\
Fe $\beta$ & $1 s 2 s^{2} 2 p^{1} P_{1} \rightarrow 1 s^{2} 2 s^{2}{ }^{1} S_{0}$ & $1.87035(8)$ & $6628.93(29)$ \\
Fe z & $1 s 2 s^{3} S_{1} \rightarrow 1 s^{2} S_{0}$ & $1.86812(11)$ & $6636.84(39)$ \\
\hline
\end{tabular}

\subsubsection{Inter-order calibration}

An inter-order comparison of spectra has to account for

- the parallax effects in the position sensitive detector due to the different mean ionization depth.

- the differences in the diffraction images due to the different crystal response, and

- the change in the refractive index of the crystal material.

The mean ionization depth $z_{i o n}$ is basically the average value of the probability of absorption $P_{a b s}(z)$ of the $\mathrm{x}$-ray photon with respect to the path of penetration $z$. The absorption probability as a function of penetration depth, $P_{a b s}(z)$, is the derivative of the fractional absorption $A=1-e^{-\mu z}$ introduced in eq.(5.2) on page 69, i.e,

$$
P_{a b s}(z)=\frac{d}{d z} A=\mu e^{-\mu z},
$$


where $\mu$ is the linear attenuation of the material. Having a detector of thickness $d_{d e t}$, the mean ionization depth is

$$
z_{i o n}=\frac{\int_{0}^{d_{d e t}} P_{a b s}(z) z d z}{\int_{0}^{d_{d e t}} P_{a b s}(z) d z}=\frac{e^{-\mu d_{d e t}}\left(\mu d_{d e t}+1\right)-1}{\mu\left(e^{-\mu d_{d e t}}-1\right)},
$$

Taking the mass attenuation $(\mu / \rho)$ for $\mathrm{Xe}, \mathrm{C}$, and $\mathrm{H}$, the detector gas mixture of $70 \% \mathrm{Xe}$, and $30 \% \mathrm{CH}_{4}$, the gas pressure of 6.4 bar, and the detector thickness of $4 \mathrm{~mm}$, a mean ionization depth of $z_{i o n}=0.593(3) \mathrm{mm}$ for the $6.5-\mathrm{keV} \times$ rays and $z_{i o n}=1.658(2) \mathrm{mm}$ for the $13-\mathrm{keV} \times$ rays can be derived. Despite this difference the line shift relative to the krypton lines due to parallax effects is less than $8 \mu \mathrm{m}$ for the $\mathrm{Mn} \mathrm{Ly}-\alpha_{1}$ lines and less than $7 \mu \mathrm{m}$ for the $\mathrm{Fe} \mathrm{K} \alpha$ transitions. For both, manganese and the iron spectra, parallax shifts the lines away from the center of the detector. This spatial shift of the calibration lines changes the energy of the krypton $1 s 2 p^{1} P_{1} \rightarrow 1 s^{2}{ }^{1} S_{0}$ transition by $-0.02 \mathrm{eV}$ and the krypton $1 s 2 s^{3} S_{1} \rightarrow 1 s^{2}{ }^{1} S_{0}$ transition by $+0.03 \mathrm{eV}$. The parallax effects, thus, are small especially when compared to the overall uncertainties of the present measurement. Adjustments for these effects, however, have been made. The reason for such small effects is the large distance between the source and the detector.

Another important issue when using different orders of diffraction is the comparison of the diffraction images. Proper determination of the center of gravity of the spectral line is of utmost importance for a successful determination of the relative line positions. As already mentioned, the line shape for the spectra taken in second order Bragg reflection is dominated by the Doppler broadening. The width of the spectral lines diffracted in first order is limited by the resolving power of the crystal. In fact, the line profile of the spectral lines measured in first order Bragg reflection is slightly asymmetric. Therefore, applying a symmetrical fit function does not give the center of gravity of the spectral line. For example, for the $M n$ Ly- $\alpha_{1}$ line this difference between the center of gravity and the centroid, obtained by using a symmetric fit function is $0.01 \mathrm{eV}$. Accounting for this asymmetry of the spectral lines in first order shifts the measured transition energies of the krypton lines on average by $0.02 \mathrm{eV}$ 
towards lower energies. Assigning a $100 \%$ uncertainty to this shift is a rather conservative measure. In comparison with the overall uncertainty of the measurement, however, this slight shift due to an asymmetric line profile is insignificant.

By contrast, the impact of the difference in the index of refraction on the transitionenergy determination turns out to be significant. The quantity of interest is the refractivity $\delta$ in eq.(5.10). The refractivity was calculated using an approximation of the dispersion formula for photon energies much higher than the resonance transitions of the medium, e.g., |114|. Modification with respect to the SI units leads to following expression for the refractivity:

$$
\delta=\frac{N_{e} e^{2} \lambda^{2}}{8 \pi^{2} \epsilon_{o} m_{e} c^{2}}
$$

$N_{e}$ is the electron density in the crystal, $e$ the elementary charge, $\epsilon_{0}$ the permittivity of vacuum, and $m_{e}$ the electron rest mass. Thus, the expression $\delta / \lambda^{2}$ is a constant factor. Taking a lattice spacing of $2 d_{\infty}=4.027 \AA$ for the $\operatorname{LiF}$ (200) crystal eq.(6.3) gives a $2 \mathrm{~d}$ spacing of $2 \mathrm{~d}=4.02678 \AA$ for first order and $2 \mathrm{~d}=4.02695 \AA$ for second order. The difference of the index of refraction for the two orders, consequently, has a significant effect on the determination of the energy of second-order lines that are calibrated by lines measured in first order. If neglected, the energy of the second-order lines are $0.54 \mathrm{eV}$ higher than if taken into account. Uncertainties in the determination of $2 d_{1}$ and $2 d_{2}$ arise from the assumed values of $2 d_{\infty}$ and $\delta / \lambda^{2}$. An estimate for the uncertainty of the former can be given based on the $0.1-\mathrm{m} \AA$ spread of values cited by different authors $[108,109]$. A $0.1 \%$ change $(0.4 \mathrm{~m} \AA)$ affects the measured krypton line energies by no more than $0.02 \mathrm{eV}$. A $10 \%$ change in the value of $\delta / \lambda^{2}$, which is much higher than expected from basic theoretical considerations [114], affects the measured krypton line energies by only $0.04 \mathrm{eV}$.

\subsubsection{Results and brief discussion}

Table 6.2 presents the results of the energy determination of the heliumlike lines. 
The uncertainty in the determination of the transition energies results from the uncertainty of the calibration lines, the uncertainty of the dispersion along the detector, the uncertainty in the determination of the centroid of each line, which includes the uncertainty due to the different line profiles in the first-and second-order spectra, as well as the uncertainty in the assumed values for $2 d_{1}$ and $2 d_{2}$. The uncertainty in the energy of the calibration lines is $0.4 \mathrm{ppm}$ for the $\mathrm{Mn} \mathrm{Ly}-\alpha_{1}$ line, $59 \mathrm{ppm}$ for the line $\mathrm{z}$ in heliumlike iron, and $43 \mathrm{ppm}$ for the berylliumlike transition $\beta$. Including the uncertainty of the determination of the centroid of these calibration lines which depends mainly on counting statistics, gives $7 \mathrm{ppm}, 59 \mathrm{ppm}$ and $44 \mathrm{ppm}$, respectively. As a result, the overall uncertainty of the krypton lines, which includes the uncertainties of the calibration lines, of the dispersion, and of the position of the centroid of the measured lines, ranges from 28 to $31 \mathrm{ppm}$.

The comparison of the results in Table 6.2 shows that the present measurement is in excellent agreement with the most recent theoretical calculation, i.e., the configuration interaction calculations performed by K.T. Cheng et al. [100]. Thus, the only verification with respect to the earlier measurement by Indelicato et al. [107] is that the average difference between measured and predicted values is biggest for the comparison with Drake's predictions [36].

The uncertainty of the measured values is too large to be able to distinguish definitively between the predictions made by Plante et al. [37] and Cheng et al. $[115,100 \mid$. For that purpose the uncertainty has to be less than $0.15 \mathrm{eV}$ or $12 \mathrm{ppm}$. The accuracy of the present measurements was limited mainly by the uncertainty of the $\mathrm{Fe}^{24+}$ and $\mathrm{Fe}^{22+}$ calibration lines (59 and $43 \mathrm{ppm}$, respectively). As more accurate values for the iron lines become available, the accuracy of the present technique will improve and be limited by statistical considerations. Such considerations limit the accuracy of the present measurements to $10-15 \mathrm{ppm}$. 
Table 6.2: Experimental and theoretical values for the energies of the $1 s 2 s, 1 s 2 p \rightarrow$ $1 s^{2}$ transitions in heliumlike krypton and average difference between experimental results and the calculated values. $E_{\text {exp }}$ denotes the result of the present measurement, $E_{I n d}$ the values measured by Indelicato et al. [107]. The theoretical predictions listed for comparison are taken from Drake $E_{D}[36]$, Plante et al. $E_{P}[37]$, and Chen et al. and Cheng et al. $E_{C \& C}[115,100]$. For the average difference, the symbol $E_{\text {theo }}$ stands for the theoretical value of the according column.

\begin{tabular}{lcccccc}
\hline \hline & & $\mathrm{E}_{\text {exp }}$ & $\mathrm{E}_{\text {Ind }}$ & $\mathrm{E}_{D}$ & $\mathrm{E}_{P}$ & $\mathrm{E}_{C \& C}$ \\
Key & Transition & $(\mathrm{eV})$ & $(\mathrm{eV})$ & $(\mathrm{eV})$ & $(\mathrm{eV})$ & $(\mathrm{eV})$ \\
$\mathrm{w}$ & $1 s 2 p^{1} P_{1} \rightarrow 1 s^{2}{ }^{2} S_{0}$ & $13114.68(36)$ & $13115.45(30)$ & 13114.33 & 13114.41 & 13114.70 \\
$\mathrm{x}$ & $1 s 2 p^{3} P_{2} \rightarrow 1 s^{2} S_{0}$ & $13091.17(37)$ & - & 13090.72 & 13090.79 & 13091.10 \\
$\mathrm{y}$ & $1 s 2 p^{3} P_{1} \rightarrow 1 s^{2} S_{0}$ & $13026.29(36)$ & $13026.80(30)$ & 13025.99 & 13026.05 & 13026.32 \\
$\mathrm{z}$ & $1 s 2 s^{3} S_{1} \rightarrow 1 s^{2}{ }^{2} S_{0}$ & $12979.63(41)$ & - & 12979.13 & 12979.20 & 12979.51 \\
Average Difference: & $\left\langle\mathrm{E}_{\text {Ind }}-\mathrm{E}_{\text {theor }}\right\rangle(\mathrm{eV})$ & $0.97(30)$ & $0.90(30)$ & $0.60(30)$ \\
& & $\left\langle\mathrm{E}_{\text {exp }}-\mathrm{E}_{\text {theor }}\right\rangle(\mathrm{eV})$ & $0.40(38)$ & $0.33(38)$ & $0.03(38)$
\end{tabular}

\subsection{Dielectronic recombination lines in the $\mathrm{K}$-shell spectra of highly charged krypton}

Initial experiments with krypton on the TFTR established the feasibility of using the emission of highly charged krypton for plasma diagnostic purposes and suggested that the $1 s 2 s^{3} S_{1} \rightarrow 1 s^{2}{ }^{1} S_{0}$ transition, i.e., line $\mathrm{z}$, in heliumlike krypton might be the most reliable indicator of the ion temperature. The suggestion of using the line $\mathrm{z}$ for the determination of the thermal line broadening is based on its supposedly low or almost negligible interference with respect to satellite lines $|116,103|$. Since a separation of direct excitation (DE) and dielectronic recombination (DR) processes is impossible in plasmas, a experimental determination of the fractional contribution of the satellite 
lines depends on electron beam devices, such as the electron beam ion trap where the electron energy distribution is extremely narrow, and allows the separation of processes that show a strong dependence on the excitation energy.

\subsubsection{Experimental setup and measurement}

The experimental setup was identical to the arrangement used for the wavelengths and transition energy measurements and, thus, a detailed description regarding the spectrometer geometry, the detector performance, and the resolving power of the setup is given in Section 6.1.1.

The output of a high-precision function generator controls the drift tube potential, i.e., the acceleration potential of the electron beam ${ }^{30}$. With a simple sawtooth pattern, a sweep over the energy interval of interest is performed. Each detected $x$-ray photon is tagged with two parameters. One is the location on the detector which is a measure of the $\mathrm{x}$-ray energy. The other parameter is the position with respect to the sawtooth function which is a measure of the electron beam energy. The spectra recorded during the sweep are, thus, three-parameter plots, with the third parameter being the number of counts. Figure 6.3 shows a set of such three-parameter spectra. In particular, it shows the result of sweeping from $11 \mathrm{keV}$ to $14 \mathrm{keV}$ beam energy, Figure (a), including the DE regime and all DR transitions from threshold down to the KLM resonances, i.e., the DR lines which have a $1 s 2 s 3 l$ excited configuration, with $l$ being the angular momentum quantum number. The sweep over the KLL resonances, Figure (b) was done separately for gaining a better resolution with respect to the beam energy - note the difference in the beam energy scales between the two spectra in Figure 6.3. The strongest lithiumlike DR satellite lines, $e, j$, and $k$ are labeled for reference purposes only. Using these kind of plots a separation of lines with the same $\mathrm{x}$-ray energy but different excitation energies is feasible.

The well resolved KLL spectrum nicely shows that comparable transitions, i.e.,

\footnotetext{
${ }^{30}$ EBIT's electron gun cathode is at ground potential.
} 

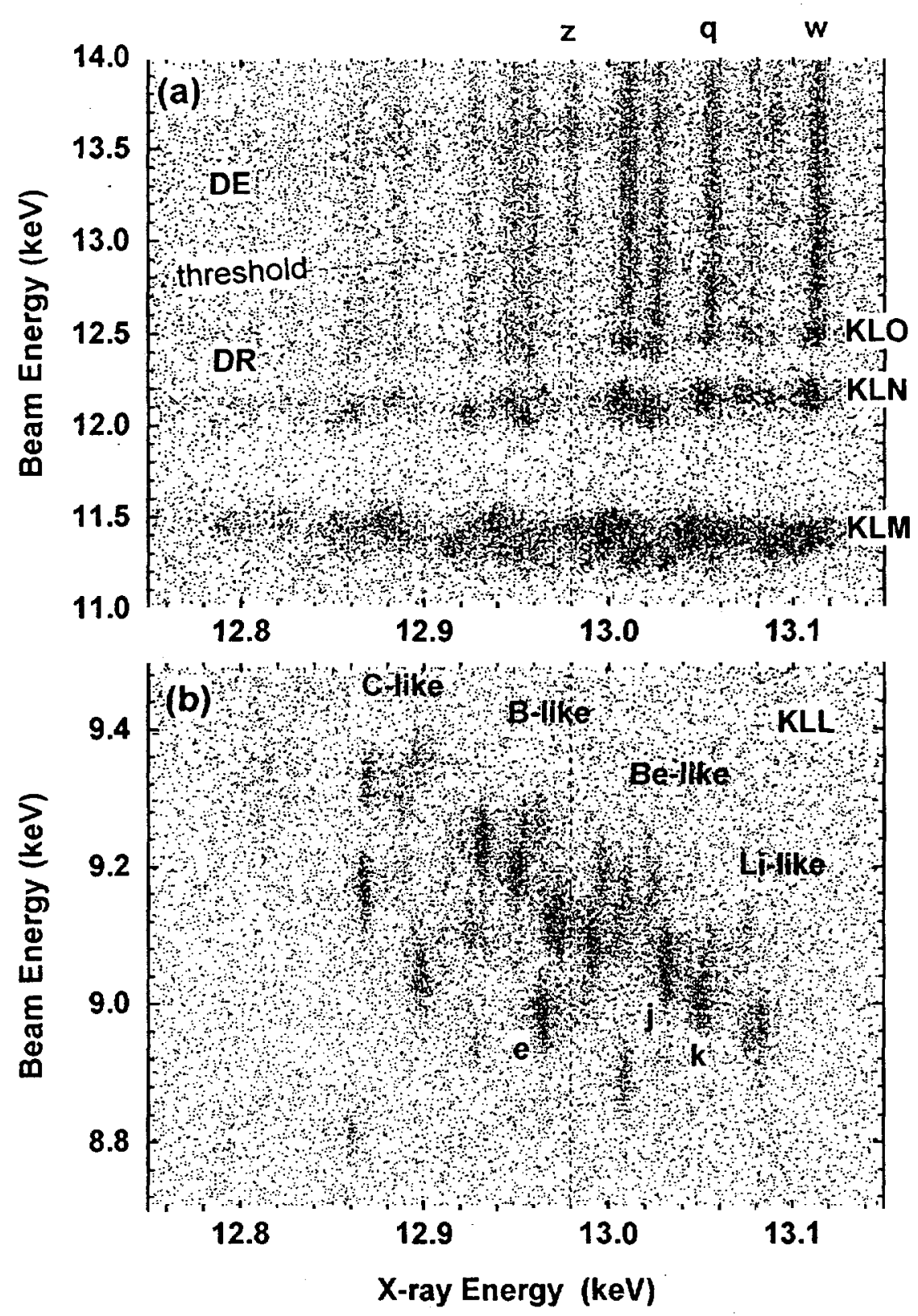

Figure 6.3: Direct excitation and dielectronic recombination lines in highly charged krypton. (a) shows all $K-$ shell $D R$ resonance lines involving $n \geq 3$ levels. The inclined dashed line marks the location where the beam energy equals the $x$-ray energy, i.e., the threshold for direct excited transitions. The notation KLM, KLN, refers to the configuration in the recombined ion prior to radiative relaxation. The sweep over the KLL resonance lines is shown in (b). The labels denote the isoelectronic sequence after the recombination process. The strongest lithiumlike DR lines are labeled, e, $\mathrm{j}$, and $\mathrm{k}$ (see Figure 6.4). The vertical dashed line traces the $\mathrm{x}$-ray. energy of the heliumlike line $z$. 
transitions involving the same subshells, in the differently charged ions are along a path of negative slope, which is somewhat indicated by the labels denoting the isoelectronic series of the highly charged krypton. This negatively sloped path is expected because the ionization potential decreases with increasing number of electrons and a higher electron beam energy is required for the resonant excitation of the innershell transition. Additionally, the energy of the emitted $x$-ray photon is shifted towards lower energies with respect to the comparable transition prior to the recombination process. Using theoretical predictions of the ionization potential for each level of the various krypton ions the $\mathrm{DR}$ resonance lines can be identified. The identification of the lithiumlike krypton KLL resonance lines is shown in Figure 6.4(a). Once the different satellite lines are identified, spectra from only one ionization state can be obtained by isolating the specific areas and projecting them onto the $\mathrm{x}$-ray energy axis. The result of cutting out only the lithiumlike krypton satellite lines, for example, leads to the spectrum shown in Figure 6.4(b).

From this spectrum the wavelength for most of the lithiumlike satellite lines are determined and the result is presented in Table 6.3.

The heliumlike transitions present in the DE spectra were used for the calibration of the spectrometer setup. The values for the heliumlike transitions were taken from the previous measurement, see Table 6.2 on page 117. The uncertainty in the determination of the wavelength and the transition energies, respectively, due to the uncertainty in the determination of the center position of each line and of the dispersion, is about $20-130 \mathrm{ppm}$. An additional uncertainty of about $30 \mathrm{ppm}$ has to be added due to the uncertainty of the wavelengths/transition energies of the calibration lines, i.e., the heliumlike $n=2 \rightarrow 1$ transitions in heliumlike krypton. The experimental values are compared to calculations by Osterheld utilizing the HULLAC code ${ }^{31}$ (private communication) and to calculations by Vainshtein and Safronova [117].

\footnotetext{
${ }^{31}$ HULLAC stands for Hebrew University Lawrence Livermore Atomic Code;
} 


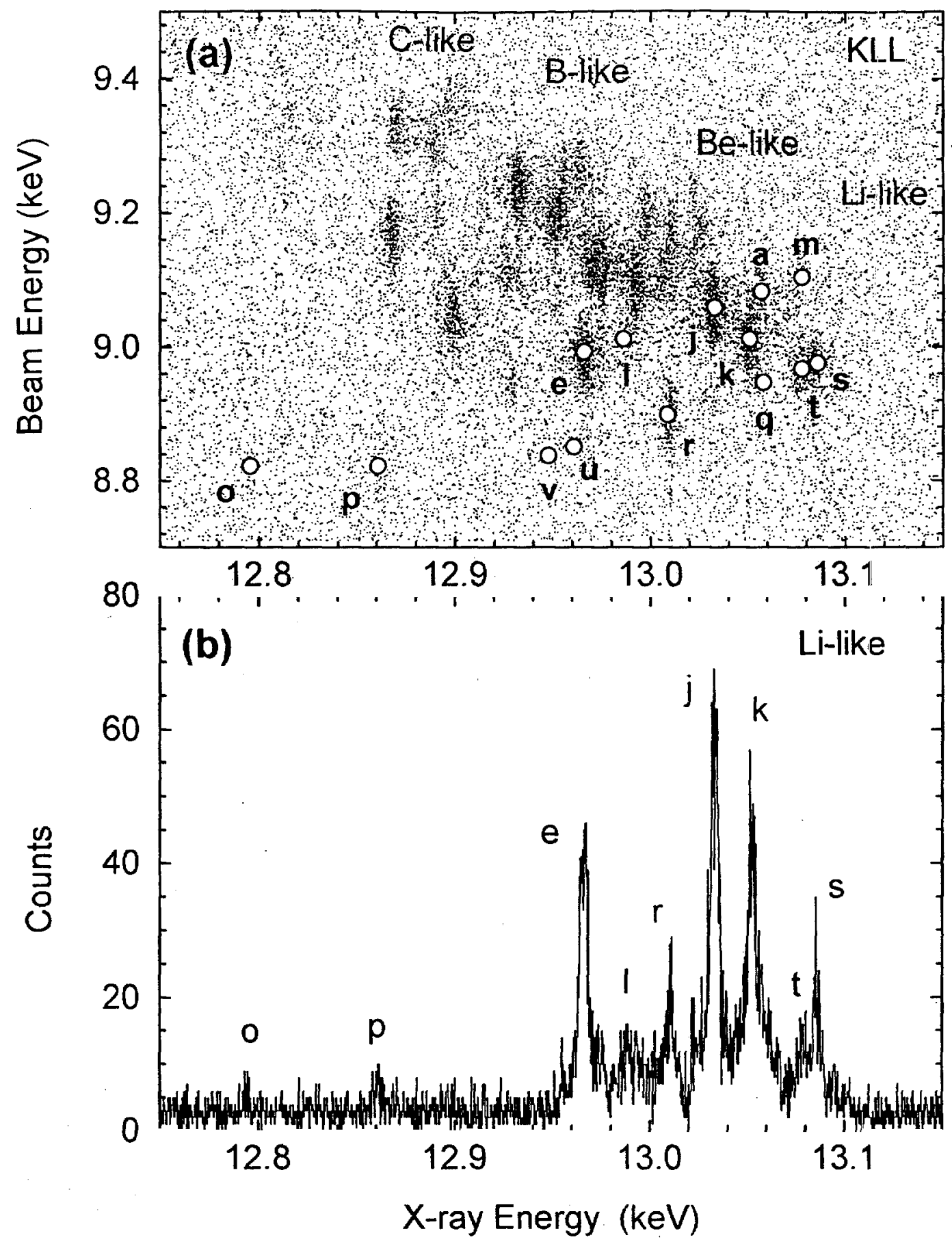

Figure 6.4: (a) Identification of the lithiunlike krypton KLL DR resonance lines using theoretical predictions of the ionization potential and the expected transition energies of these DR satellite lines. The spectrum (b) was obtained by projecting the recorded spectrum onto the $\mathrm{x}$-ray energy axis after "cutting" around the lithiumlike resonances . Thus, the spectrum only contains the contributions of the lithiumlike krypton DR resonances. 
Table 6.3: Comparison between the measured and some calculated wavelengths values of the $\mathrm{DR}$ satellite lines of lithiumlike $\mathrm{Kr}^{33+}$. The spectra were calibrated using the measured values of the heliumlike lines ( $w, x, y$, and $z$ ) listed in Table 6.2. The subscript exp denotes the measured values, $v \& s$ theoretical predictions by Vainshtein and Safronova [117], and $o$ denotes present calculations by Al Osterheld (see text).

\begin{tabular}{|c|c|c|c|c|}
\hline Key & Transition & $\begin{array}{l}\lambda_{\exp } \\
(\AA)\end{array}$ & $\begin{array}{c}\lambda_{V \& S} \\
(\AA)\end{array}$ & $\begin{array}{l}\lambda_{0} \\
(\AA)\end{array}$ \\
\hline $\mathbf{w}$ & $1 \mathrm{~s} 2 \mathrm{p}^{1} \mathrm{P}_{1} \rightarrow 1 \mathrm{~s}^{2}{ }^{1} \mathrm{~S}_{0}$ & $0.945385(26)$ & 0.94538 & 0.94536 \\
\hline $\mathrm{x}$ & $1 \mathrm{~s} 2 \mathrm{p}^{3} \mathrm{P}_{2} \rightarrow 1 \mathrm{~s}^{2}{ }^{1} \mathrm{~S}_{0}$ & $0.947083(27)$ & 0.94708 & 0.94709 \\
\hline s & $1 s 2 p 2 s\left({ }^{3} P\right)^{2} P_{3 / 2} \rightarrow 1 s^{2} 2 s^{2} S_{1 / 2}$ & $0.94758(3)$ & 0.94746 & 0.94746 \\
\hline $\mathrm{t}$ & $1 s 2 p 2 s\left({ }^{3} P\right)^{2} P_{1 / 2} \rightarrow 1 s^{2} 2 s^{2} S_{1 / 2}$ & $0.94808(4)$ & 0.94804 & 0.94804 \\
\hline q & $1 s 2 p 2 s\left({ }^{1} P\right)^{2} P_{3 / 2} \rightarrow 1 s^{2} 2 s^{2} S_{1 / 2}$ & $0.94962(5)$ & 0.94961 & 0.94949 \\
\hline k & $1 s 2 p^{22} D_{3 / 2} \rightarrow 1 s^{2} 2 p^{2} P_{1 / 2}$ & $0.95000(2)$ & 0.94995 & 0.94500 \\
\hline $\mathrm{j}$ & $1 s 2 p^{22} D_{5 / 2} \rightarrow 1 s^{2} 2 p^{2} P_{3 / 2}$ & $0.95141(2)$ & 0.95137 & 0.95124 \\
\hline $\mathbf{y}$ & $1 \mathrm{~s} 2 \mathrm{p}^{3} \mathrm{P}_{1} \rightarrow 1 \mathrm{~s}^{2}{ }^{1} \mathrm{~S}_{0}$ & $0.951800(26)$ & 0.95156 & 0.95175 \\
\hline $\mathrm{r}$ & $1 s 2 p 2 s\left({ }^{1} P\right)^{2} P_{1 / 2} \rightarrow 1 s^{2} 2 s^{2} S_{1 / 2}$ & $0.95310(5)$ & 0.95288 & 0.95306 \\
\hline$\beta$ & $1 s 2 p 2 s^{21} P_{1} \rightarrow 1 s^{2} 2 s^{2}{ }^{1} S_{0}$ & $0.95312(12)$ & & 0.95350 \\
\hline 1 & $1 s 2 p^{2} D_{3 / 2} \rightarrow 1 s^{2} 2 p^{2} P_{3 / 2}$ & $0.95471(5)$ & & 0.95475 \\
\hline $\mathrm{z}$ & $1 \mathrm{~s} 2 \mathrm{p}^{3} \mathrm{~S}_{1} \rightarrow 1 \mathrm{~s}^{2} \mathrm{~S}_{0}$ & $0.955222(30)$ & 0.95525 & 0.95519 \\
\hline e & $1 s 2 p^{2} P_{5 / 2} \rightarrow 1 s^{2} 2 p^{2} P_{3 / 2}$ & $0.95637(2)$ & 0.95615 & 0.95623 \\
\hline $\mathrm{p}$ & $1 s 2 s^{2} S_{1 / 2} \rightarrow 1 s^{2} 2 p^{2} P_{1 / 2}$ & $0.96415(4)$ & & 0.96403 \\
\hline o & $1 s 2 s^{2}{ }^{2} S_{1 / 2} \rightarrow 1 s^{2} 2 p^{2} P_{3 / 2}$ & $0.96900(7)$ & & 0.96893 \\
\hline
\end{tabular}




\section{The $\mathrm{K} \alpha$ emission of heliumlike $\mathrm{Xe}^{52+}$ and hydro- genlike $\mathrm{Xe}^{53+}$}

Next generation fusion devices, such as ITER or NIF, are proposed to have electron temperatures in excess of $30 \mathrm{keV}^{32}$. In such a high electron temperature environment bare ions are the most abundant species among low- and mid- $\mathrm{Z}$ elements up to $\mathrm{Z} \approx$ 30. Thus, reliable measurements of the central ion temperature in such hot plasmas require trace amounts of higher $-\mathrm{Z}$ material in the plasma core region. A brief list of the ionization potentials for the lithium-, helium-, and hydrogenlike isoelectronic sequence for some elements is given in Table 7.1.

Table 7.1: Ionization potentials $U_{i o n}$ of some elements for the lithiumlike, heliumlike, and hydrogenlike isoelectronic sequence. The values were calculated by Scofield [101] and represent the binding potential of the ground state electron. For example, an energy of $12.3889 \mathrm{keV}$ is required to remove the electron from the ground state of hydrogenlike $\mathrm{Zn}^{29+}$.

\begin{tabular}{cccccc}
\hline \hline isoelectronic & & \multicolumn{5}{c}{$U_{\text {ion }}(\mathrm{kV})$} \\
sequence & $\mathrm{Zn}(\mathrm{Z}=30)$ & $\mathrm{Kr}(\mathrm{Z}=36)$ & $\mathrm{Mo}(\mathrm{Z}=42)$ & $\mathrm{Ag}(\mathrm{Z}=47)$ & $\mathrm{Xe}(\mathrm{Z}=54)$ \\
H-like & 12.3889 & 17.9361 & 24.5721 & 30.9656 & 41.2996 \\
He-like & 11.8648 & 17.2962 & 23.8103 & 30.0969 & 40.2711 \\
Li-like & 2.7820 & 4.1091 & 5.7132 & 7.2713 & 9.8104
\end{tabular}

The elements of choice for admixing to the fusion plasma are noble gases because of their convenient handling regarding the introduction into as well as the removal from the plasma devices. Thus, the prime candidates are krypton and xenon. The

\footnotetext{
${ }^{32}$ It is common practice in the field of plasma physics to express temperatures in energy units. Correctly, one should speak of thermal energies $E_{t h}=k_{B} T$, where $T$ is the temperature, and $k_{B}$ the Boltzmann constant: $\frac{1}{k_{B}}=11604.448(99) \frac{\mathrm{K}}{\mathrm{eV}}$.
} 
ion species of choice for ion temperature diagnostics utilizing $\mathrm{x}$-ray spectroscopy are the members of the heliumlike isoelectronic sequence (see also Chapter 2).

The ion temperature diagnostic is based on the measurement of the Doppler broadening of the spectral line. Thus, reliable determination of the ion temperature requires a minimum spectral resolving power so that the characteristic emission is not dominated by the apparatus profile. Figure 2.1 (page 8) shows that for krypton, for example, the minimum resolving power is below 1000 for ion temperatures above $15 \mathrm{keV}$. Employing xenon increases the minimum resolving power to about 1100 for the same ion temperature. However, using xenon extends the accessible ion temperature range far beyond $30 \mathrm{keV}$, a regime in which krypton would be already fully stripped. Currently, spectroscopic investigation of the $\mathrm{K} \alpha$ emission of highly charged ions heavier than krypton, i.e., $\mathrm{Z}>36$, has been limited to low-resolution instruments, such as germanium detectors which have resolving powers below 110 [78|. Therefore, extending high-resolution measurements of the $\mathrm{K} \alpha$ emission of highly charged ions to the high- $\mathrm{Z}$ elements and, hence, to the hard $\mathrm{x}$-ray regime was the goal of following experimental effort.

Section 7.1 presents the high-resolution measurements of the $\mathrm{K} \alpha$ emission of heliumlike $\mathrm{Xe}^{52+}$, and hydrogenlike $\mathrm{Xe}^{53+}$ utilizing a transmission-type crystal spectrometer. The fine-structure resolved spectrum of the $n=2 \rightarrow 1$ transitions allows a charge-specific determination of the line intensities. From the measured relative intensities the electron-impact excitation cross sections are inferred relative to the cross sections for the radiative recombination lines. The uncertainties of the measured cross sections are in the $15 \%$ to $35 \%$ range, and are mostly due to counting statistics. A systematic uncertainty of about $3 \%$ arises from the radiative recombination cross sections values. For the hydrogenlike transitions agreement with theoretically predicted values of the electron-impact excitation cross sections has been found. For some heliumlike transitions, however, experimentally and theoretically achieved values disagree significantly, indicating the importance of including the Breit interaction 
in the atomic models. The wavelengths of the $n=2 \rightarrow 1$ transitions were determined relative to the $L y-\alpha_{1}$ transition. Thus, the calibration of the highly resolved xenon spectrum depends on a high-precision measurement of the Ly- $\alpha_{1}$ wavelength or its transition energy with respect to the primary $\mathrm{x}$-ray standard, i.e., the tungsten $\mathrm{K}-\alpha_{1}$ transition. Such a measurement is presented in Section 7.2.

\subsection{Transmission-type crystal spectrometer measurements}

The $\mathrm{K} \alpha$ transitions in heliumlike, and hydrogenlike xenon $\left(\mathrm{Xe}^{52+}\right.$ and $\left.\mathrm{Xe}^{53+}\right)$ have been measured at the SuperEBIT facility using a transmission-type crystal spectrometer. The resolving power of this spectrometer is sufficient for level-specific measurements of the $n=2 \rightarrow 1$ transitions. The spectral region observed includes the heliumlike, and hydrogenlike transitions listed in Table 7.2.

\subsubsection{Experimental setup}

A transmission-type crystal spectrometer was designed, based on the DuMond geometry [94], for implementation at the SuperEBIT facility. The spectrometer contains a $(140 \times 40 \times 0.6) \mathrm{mm}^{3}$ quartz crystal with a $(13 \overline{4} 0)$ lattice plane orientation which is cylindrically bent. The reflecting lattice planes are parallel to the axes of this cylinder and perpendicular to the surface of the crystal. The DuMond geometry requires that the radius of curvature of the crystal is the diameter of the so-called Rowland circle (see Fig. 5.9 on page 94). For optimized throughput per wavelengths interval of the transmitted $\mathrm{x}$-rays the spectrometer has to be adjusted in such a way that the electron beam lies on the Rowland circle, and is parallel to the diffracting lattice planes of the crystal. An optimized setup also minimizes the energy band width of the in transmission diffracted $x$ rays. A simulation of the energy distribution of the diffracted $x$ rays depending on the geometric quantities of the experimental setup is presented in Figure 5.11 and Figure 5.12. The setup is sketched in Figure 7.1. Table 7.3 presents a list of some geometric quantities associated with the spectrom- 
Table 7.2: Predicted values for the $K \alpha$ transition energies $E$ and wavelengths $\lambda$ of hydrogenlike, heliumlike, and neutral xenon. The values for the hydrogenlike transitions are taken from Johnson and Soff [27]. Drake [36] was used as a reference for the heliumlike transitions. The lithiumlike transition $\mathrm{q}$ was calculated by $\mathrm{M}$. Chen using a multi-configuration-Dirac-Fock (MCDF) code (private communication). The transitions of neutral xenon, $K \alpha_{1}$ and $K \alpha_{2}$, were taken from Mooney et al. $[118]$ and the spectroscopic notation describes the location of the electron hole.

\begin{tabular}{lcccc}
\hline \hline ion & transition & name & $\begin{array}{c}E \\
\mathrm{eV}\end{array}$ & $\begin{array}{c}\lambda \\
\mathrm{pm}\end{array}$ \\
\hline $\mathrm{Xe}^{53+}$ & $2 p_{3 / 2} \rightarrow 1 s_{1 / 2}$ & $\mathrm{Ly}-\alpha_{1}$ & 31283.8 & 39.6321 \\
$\mathrm{Xe}^{53+}$ & $2 p_{1 / 2} \rightarrow 1 s_{1 / 2}$ & $\mathrm{Ly}-\alpha_{2}$ & 30856.4 & 40.1810 \\
$\mathrm{Xe}^{53+}$ & $2 s_{1 / 2} \rightarrow 1 s_{1 / 2}$ & $\mathrm{Ly}-\alpha_{3}$ & 30863.5 & 40.1718 \\
$\mathrm{Xe}^{52+}$ & $1 s 2 p^{1} P_{1} \rightarrow 1 s^{2}{ }^{1} S_{0}$ & $\mathrm{w}$ & 30629.3 & 40.4790 \\
$\mathrm{Xe}^{52+}$ & $1 s 2 p^{3} P_{2} \rightarrow 1 s^{2}{ }^{1} S_{0}$ & $\mathrm{x}$ & 30593.5 & 40.5263 \\
$\mathrm{Xe}^{52+}$ & $1 s 2 p^{3} P_{1} \rightarrow 1 s^{2}{ }^{1} S_{0}$ & $\mathrm{y}$ & 30205.6 & 41.0468 \\
$\mathrm{Xe}^{52+}$ & $1 s 2 s^{3} S_{1} \rightarrow 1 s^{2}{ }^{1} S_{0}$ & $\mathrm{z}$ & 30128.4 & 41.1520 \\
$\mathrm{Xe}^{51+}$ & $1 s 2 s 2 p^{2} P_{3 / 2} \rightarrow 1 s^{2} 2 s^{2} S_{1 / 2}$ & $\mathrm{q}$ & 30526.7 & 40.6150 \\
$\mathrm{Xe}$ & $1 s_{1 / 2}^{-1} \rightarrow 2 p_{3 / 2}^{-1}$ & $\mathrm{~K} \alpha_{1}$ & $29778.69(10)$ & $41.63522(14)$ \\
$\mathrm{Xe}$ & $1 s_{1 / 2}^{-1} \rightarrow 2 p_{1 / 2}^{-1}$ & $\mathrm{~K} \alpha_{2}$ & $29458.16(5)$ & $42.08825(7)$ \\
\hline \hline
\end{tabular}

eter and of the results of the energy band width calculations. There are two sets of measurements presented here, which in following will be referred to as measurement SE28 and measurement SE37. Both measurements were performed using the Quartz crystal, which has an intrinsic resolving power in the $10^{5}$ range [108|. That is much higher than the maximum resolving power due to the geometric properties of the spectrometer setup and the electron beam, respectively (see Table 7.3). The calculation of the energy distribution of the diffracted and detected $x$ rays also allows 


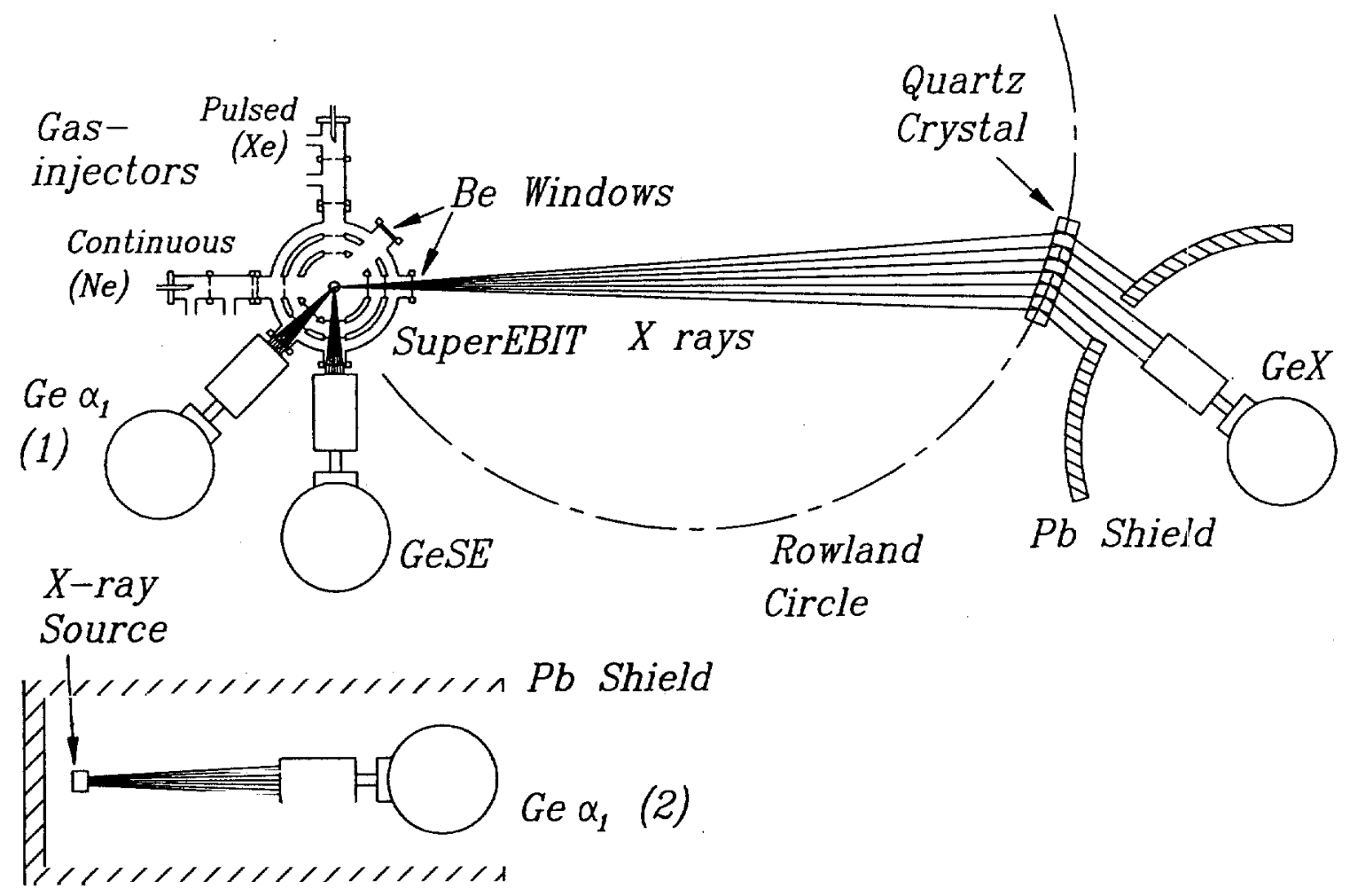

Figure 7.1: Sketch of the transmission-type crystal spectrometer setup. The electron beam, oriented perpendicular to the page, and the cylindrically bent crystal have to be part of the Rowland circle. The transmission spectrometer is operated in monochromator mode, i.e, a spectrum is obtained by rotating the crystal and archiving the number of photons detected with the germanium detector GeX as a function of the crystal position. Synchronized data acquisition between the germanium detectors GeX and GeSE is essential for the normalization of the measured high-resolution spectra. For the energy calibration of the Ly- $\alpha_{1}$ transition (see Section 7.2), xenon $\mathrm{K} \alpha$ spectra - detector $\mathrm{Ge} \alpha_{1}$ in position (1) - and calibration spectra - Ge $\alpha_{1}$ in position (2) - were taken successively. About every three hours the two detectors were interchanged.

an estimate of the impact of uncertainties in the setup geometry. The calculations show, for example, that the uncertainties of the distance between the crystal and the electron beam (quantity $D_{s}$ in Table 7.3 ) increase the energy band width by $1.1 \mathrm{eV}$ for the SE28 measurement and $1.4 \mathrm{eV}$ for the SE37 measurement. For small displace- 
ments with respect to the crystal-electron-beam distance the energy spread is, thus, roughly $0.5 \mathrm{eV}$ per $\mathrm{mm}$. This is much less than the impact of uncertainties in lateral direction, i.e., perpendicular to the emitted $\mathrm{x}$ rays. The $\approx 90-\mu \mathrm{m}$ broadening due to the electron beam diameter, for example, causes an increase of the transmitted $\mathrm{x}$-ray energy band by $6 \mathrm{eV}$, which would be $\approx 60 \mathrm{eV}$ per mm. The high sensitivity with respect to the lateral dimension makes the curvature of the crystal a very crucial parameter. The width of the image of a point source was taken as an indicator for the quality of the crystal curvature. The setup for measuring this image width is shown in Figure 7.2. No CCD camera was used for determining the image width regarding measurement SE28. Thus, the value in Table 7.3 reflects only the overall width of the image observed on the screen. For measurement SE37 the intensity profile of the image was measured with a CCD camera and the line width deducted from fitting the profile at different positions. The impact of the image qualities of the bent crystal on the resolving power - listed in Table 7.3 - clearly shows that the image width of the crystal is the limiting factor with respect to the resolution of the spectrometer.

The SuperEBIT transmission-type spectrometer was designed for operation in monochromator mode and, thus, no position sensitive detector is necessary. A highpurity germanium detector - detector GeX in Figure 7.1 - with almost $100 \%$ counting efficiency for $30 \mathrm{keV}$ photons is used instead. The energy dispersion of the germanium detector can be utilized for suppressing the background. A spectrum is obtained by rotating the crystal and archiving the number of photons detected with the germanium detector as a function of the crystal position. The crystal is rotated by means of a high-precision stepper motor which is mounted on a modified rotation stage. The stepper motor ${ }^{33}$ has a unidirectional repeatability of $\pm 1 \mu \mathrm{m}$, the uncertainty with respect to the traveled range is $\pm 5 \mu \mathrm{m}$. In combination with the $6 \frac{3}{8} "(\approx 161.9 \mathrm{~mm})$ lever of the modified rotation stage minimum angular increments

\footnotetext{
${ }^{33}$ The stepper motor, which has a $12.7-\mathrm{mm}$ range of travel; and driver (with RS-232 interface) are from ORIEL Corporation, Models 18503 and 20010, respectively.
} 
(a)

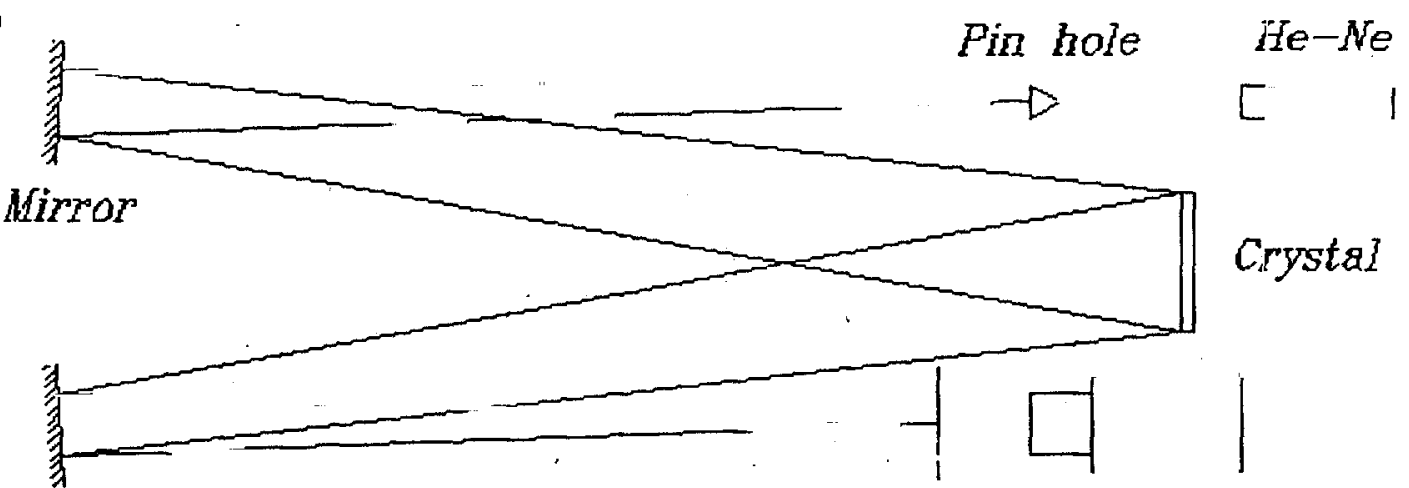

\section{Mirror}

Screen

$C C D$

(b)

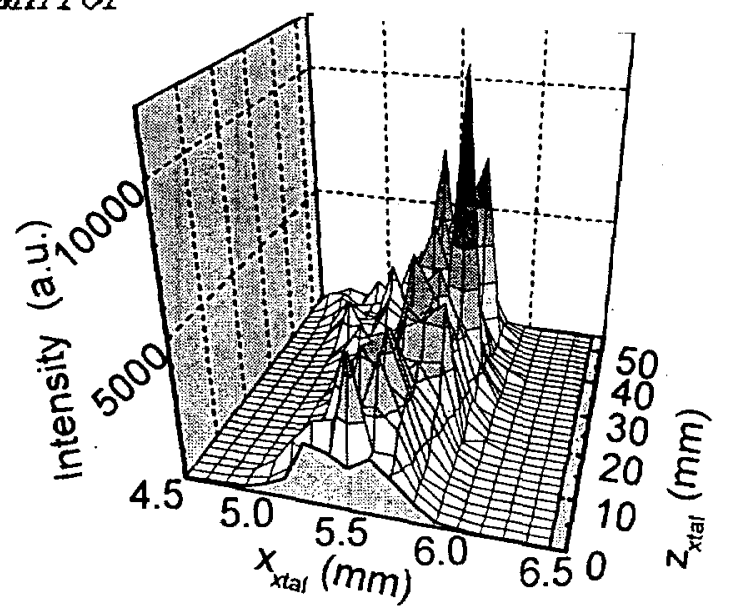

Figure 7.2: Measurement of the imaging qualities of the cylindrically bent quartz crystal. A point source ( $25 \mu \mathrm{m}$ pin hole) illuminated by a He-Ne laser was imaged by the crystal onto a screen and the intensity distribution of this image measured with a CCD camera. (a) shows the optical setup and (b) the intensity distribution of the line image. The spatial dimensions were calibrated by imaging a metal precision ruler yielding 56(1) $\mu \mathrm{m}$ per pixel. The width of the line image was $230(30) \mu \mathrm{m}$.

of $0.00035^{\circ}$ are achievable. To take advantage of this high unidirectional precision, all scans were performed by always rotating the crystal in the same manner, e.g., counter-clockwise.

Operation of the spectrometer in monochromator mode requires a normalization of the counts measured at each crystal position. The normalization which seemed best suited for these measurements is based on the total number of xenon $\mathrm{K} \alpha \mathrm{x}$ 
Table 7.3: Geometric quantities of the transmission-type spectrometer setup for the two different sets of measurements SE28 and SE37. For both measurements a quartz (13ד̈0) crystal was used which has a $2 \mathrm{~d}$ spacing of $2.3604 \AA[101] . R_{x t a l}$ is the radius of curvature of the crystal, $D_{s}$ the distance between the crystal and the electron beam, $A_{\text {eff }}$ the effective area, that is the detector area which is illuminated by the in transmission diffracted $\mathrm{x}$ rays. The quantity $\delta x_{\text {image }}$ is the width of the line received by imaging a $25 \mu \mathrm{m}$ point source with the curved crystal. The energy bandwidth $\Delta E$ of the photons hitting the area $A_{\text {eff }}$ is calculated as described in Section 5.2.3. In particular, $\Delta E_{\text {geom }}$ is the bandwidth due to the uncertainties in the adjustment of the spectrometer, $\Delta E_{\text {beam }}$ is caused by the $90-\mu \mathrm{m}$ diameter electron beam, $\Delta E_{\text {focus }}$ is due to the deviation of the crystal curvature from a perfect cylindrical shape, and $\Delta E_{\text {total }}$ combines all three.

\begin{tabular}{rcc}
\hline \hline & SE28 & SE37 \\
\hline$R_{\text {xtal }}(\mathrm{mm})$ & $2713.8 \pm 3.2$ & $2511.4 \pm 1.5$ \\
$D_{s}(\mathrm{~mm})$ & $2675 \pm 5$ & $2475 \pm 5$ \\
$A_{\text {eff }}\left(\mathrm{mm}^{2}\right)$ & 201 & 1018 \\
$\delta x_{f}(\mu \mathrm{m})$ & $<400$ & $230 \pm 30$ \\
$\Delta E_{\text {geom }}(\mathrm{eV})$ & 1.1 & 1.3 \\
$\Delta E_{\text {beam }}(\mathrm{eV})$ & 6.1 & 6.4 \\
$\Delta E_{\text {focus }}(\mathrm{eV})$ & 27 & 16 \\
$\Delta E_{\text {total }}(\mathrm{eV})$ & 28 & 18 \\
\hline \hline
\end{tabular}

rays emitted from the trap region. Thus, a second germanium detector was setup at a different SuperEBIT access port for measuring the $K \alpha$ spectrum. Due to the cylindrical symmetry of the electron beam all access ports receive the same type and amount of illumination over time which makes a normalization by means of the measured flux at a different access port feasible. When the number of xenon Ka photons measured with this germanium detector reached a preset amount the counting 
process for both detectors, the transmission spectrometer detector and the K $\alpha$ survey detector was stopped, the crystal moved into the next position, and the counting process restarted. This type of normalization automatically takes care of possible variation in the overall xenon $\mathrm{K} \alpha \mathrm{x}$-ray emission of SuperEBIT caused, for example, by high voltage breakdowns. The normalization of the transmission spectrometer counts could be also achieved by monitoring the radiative recombination (RR) lines instead of the K $\alpha$ emission. The $\mathrm{x}$-ray energies of the RR lines, however, depend on the electron beam energy and, thus, the energy gate set for counting the RR photons might have to be changed after each time the SuperEBIT device is tuned.

For the observation of the highly charged xenon emission neutral xenon atoms are injected into SuperEBIT by means of a gas injector (see Figure 7.1). The injector used in measurement SE28 supplied the xenon in form of a continuous flow. In measurement $S E 37$ a gas injector with a pulsed supersonic beam valve was used (see also Section 4.2.1). A continuous flow of neon was also introduced into the trap region in order to supply light ions for the evaporative cooling process, which is discussed in Section 4.4. The gas injector pressure for the nëon cooling was in the order of $2.6 \cdot 10^{-8}$ Torr $\left(\approx 3.5 \cdot 10^{-6} \mathrm{~Pa}\right)$. The xenon injection pressure was set to about $5 \cdot 10^{-9}$ Torr $\left(\approx 6.7 \cdot 10^{-7} \mathrm{~Pa}\right)$ in the case of the continuous flow (SE28). In the pulsed injection mode $(S E 37)$ the injection pressure was above $6 \cdot 10^{-8}$ Torr $\left(\approx 8 \cdot 10^{-6} \mathrm{~Pa}\right)$.

The duration of the timing cycle was $15.5 \mathrm{~s}$ (SE28) and $11.2 \mathrm{~s}$ (SE37), respectively. This includes injection of xenon gas, ionization until the high charge states are obtained, period of data acquisition, and dumping of the ions before other high-Z elements intrinsic to the SuperEBIT device ${ }^{34}$ accumulate in the trap. The time for dumping and injecting was $1.25 \mathrm{~s}$ (SE28) and $0.5 \mathrm{~s}$ (SE37), the ionization took about $2.0 \mathrm{~s}$ (SE28) and $2.2 \mathrm{~s}$ (SE37). There was an additional "dead time" with respect to the data acquisition since no counts were taken while the crystal was actually in

\footnotetext{
${ }^{34}$ For example, barium and tungsten from the electron gun assembly eventually reach the trap and displace lower $-\mathrm{Z}$ elements.
} 
motion. In particular, the data acquisition was stopped for $1.1 \mathrm{~s}$ in $S E 28$ and $1.8 \mathrm{~s}$ in SE37. In average, the crystal was moved every $60 \mathrm{~s}$ (SE28) and $40 \mathrm{~s}$ (SE37), respectively. Therefore, the fraction of the measuring time used for actual data acquisition was about $77 \%(12 \mathrm{~s})$ for measurement SE28 and $72 \%(8.1 \mathrm{~s})$ for measurement SE3\%.

\subsubsection{Measurements}

For both measurements the SuperEBIT operating parameters, i.e., beam current and beam energy, are listed in Table 7.4.

In measurements $S E 28$ the spectra were acquired by only recording the number of photons detected with the GeX detector (see Figure 7.1) that were within the xenon $\mathrm{K} \alpha$ emission band as a function of the crystal position. The energy gate for selection of only the $\mathrm{K} \alpha$ emission was set hardware wise. The overall spectrum is a composite of several scans over three different spectral regions, covering the transitions $\mathrm{z}$ and $\mathrm{y}$, $\mathrm{w}$, and Ly- $\alpha_{1}$ (see Table 7.2 for spectroscopic notation). In particular, 78 scans over the lines $\mathrm{z}$ and $\mathrm{y}, 69$ scans over the line $\mathrm{w}$, and 75 scans over the Ly- $\alpha_{1}$ transition have been performed. The result - already normalized with respect to the number of scans and, thus, to the overall $\mathrm{K} \alpha$ emission - is shown in Figure 7.3. The wholespectrum represents 323 hours of measurement time of which $77 \%$,i.e., 250 hours, were actual data acquisition time (see Table 7.5). The count rate for each line is listed in Table 7.5.

The data acquisition for measurement SE37 was more elaborated and allowed to archive the complete GeX, and GeSE spectra at each crystal position. Thus, the spectra are 3-dimensional data arrays: one dimension is assigned to the crystal position, and for each position there is a 2-dimensional germanium detector spectrum, i.e., $x$-ray energy versus number of counts. Such three-parameter plots are displayed in Figure 7.4 showing the transmission-type crystal spectrum recorded with the detector GeX, and in Figure 7.5 showing the overall $x$-ray emission of the trapped ions and the Bremsstrahlungskontinuum recorded with the detector GeSE. Both axes represent 
Table 7.4: Main operating parameters of the SuperEBIT device for the two different sets of measurements SE28 and SE37. $U$ is the potential difference between the electron-gun cathode and the middle drift tube electrode, $I_{\text {beam }}$ the electron beam current, $r_{b}$ the beam radius including $80 \%$ of all electrons (see eq. (4.4) on page 26 , using the same parameters as in Table 4.4 on page 44). $U_{s c}$ is the space charge potential according to eq. (4.25), page $35, \bar{U}_{s c}$ denotes the average space charge potential within the radial distance $r_{b} \sqrt{ } 1.2$, which would be the edge of the beam assuming that the electron density $n_{e}$ is homogeneous. $E_{b e a m}$ is the electron beam energy, and $v_{e}$ the velocity of the electrons given as a fraction of the speed of light. $c_{0}$. The uncertainty of $E_{\text {beam }}$ includes the difference of the space charge potential between the center and the radial distance $r_{b}$ of the beam.

\begin{tabular}{rcc}
\hline \multicolumn{3}{c}{ SuperEBIT } \\
quantity & SE28 & SE37 \\
\hline$U(\mathrm{kV})$ & $114.147(10)$ & $112.203(10)$ \\
$I_{\text {beam }}(\mathrm{mA})$ & $270(10)$ & $250(10)$ \\
$r_{b}(\mu \mathrm{m})$ & $46.4(9)$ & $46.3(9)$ \\
$U_{s c}(0 \mu \mathrm{m})(\mathrm{V})$ & $-146.32(19)$ & $-136.36(18)$ \\
$U_{s c}\left(r_{b} \sqrt{1.2}\right)(\mathrm{V})$ & $-127.88(15)$ & $-119.16(15)$ \\
$\bar{U}_{s c}(\mathrm{~V})$ & $-139.13(24)$ & $-129.68(23)$ \\
$E_{\text {beam }}(\mathrm{keV})$ & $114.008(21)$ & $112.073(20)$ \\
$v_{e}\left(c_{0}\right)$ & $0.57580(4)$ & $0.57218(4)$ \\
$n_{e}\left(\mathrm{~cm}^{-3}\right)$ & $1.15(4) 10^{12}$ & $1.08(4) 10^{12}$ \\
\hline \hline
\end{tabular}

the $\mathrm{x}$-ray energy. The number of counts, which is the third parameter, is graphically depicted by the density (and "color") of the points. This transmission-type crystal spectrum includes scans over the Ly- $\alpha_{2,3}$ transitions, a spectral region that was not observed in measurement SE28. The total data acquisition time was about 122 hours and all together 144 scans were performed, in particular, 57 scans over Ly- $\alpha_{1}, 22$ 


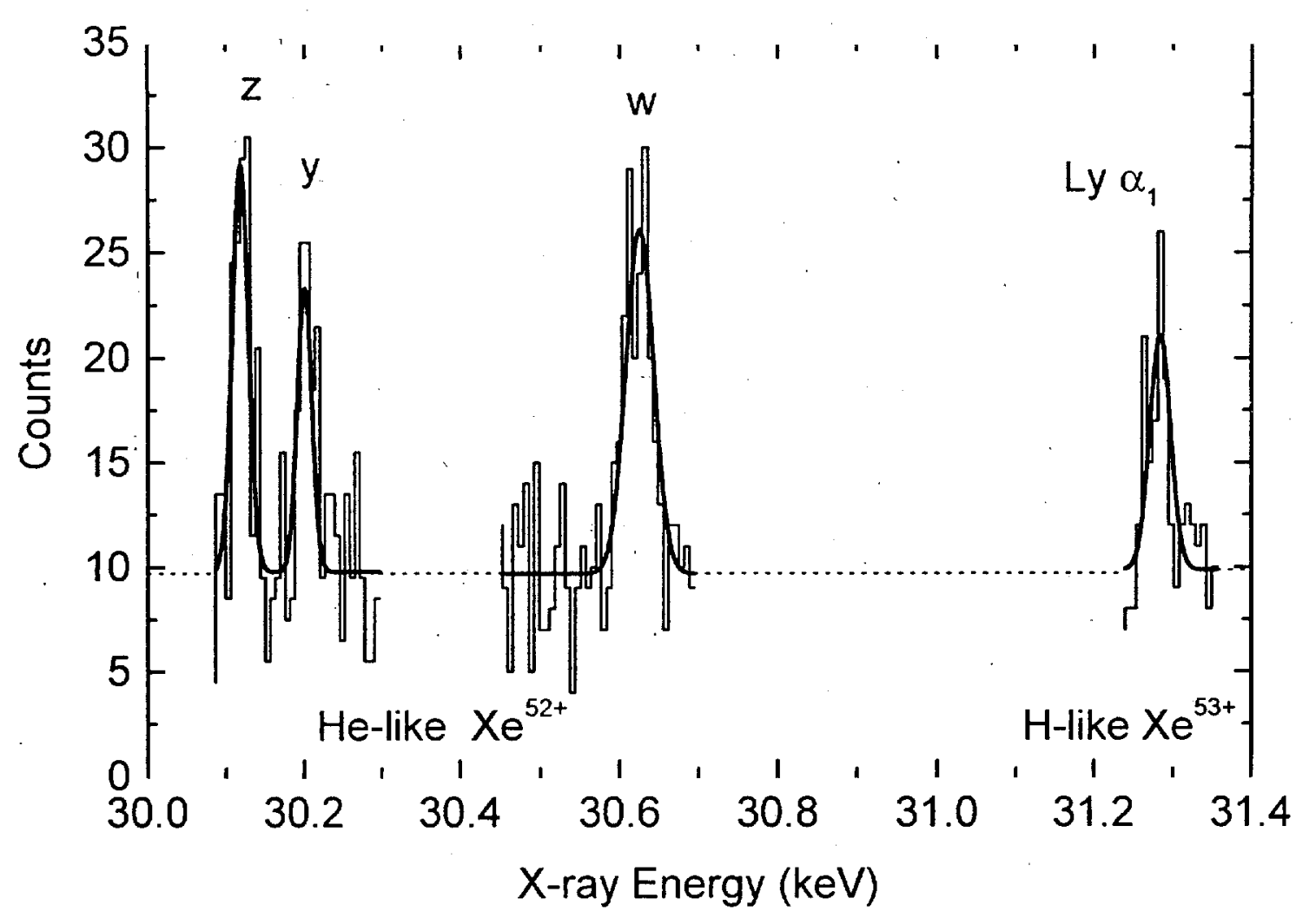

Figure 7.3: Spectrum of the $2 p_{3 / 2} \rightarrow 1 s_{1 / 2}\left(\mathrm{Ly}-\alpha_{1}\right)$ transition in hydrogenlike $\mathrm{Xe}^{53+}$, and the $1 s 2 p^{1} P_{1} \rightarrow 1 s^{21} S_{0}(\mathrm{w}), 1 s 2 p^{3} P_{1} \rightarrow 1 s^{2}{ }^{1} S_{0}(\mathrm{y})$, and $1 s 2 s^{3} S_{1} \rightarrow 1 s^{2}{ }^{1} S_{0}$ (z) transitions in heliumlike $\mathrm{Xe}^{52+}$. Each channel represents the number of detected photons accumulated over three steps, i.e., three consecutive crystal positions. From the fitted line width of $z$ and $y$ a resolving power of $1600 \pm 320$ was inferred. The dispersion is about $6.6 \mathrm{eV}$ per channel. The total data acquisition time was 250 hours.

scans over $\mathrm{Ly}-\alpha_{2,3}, 9$ scans over $\mathrm{w}$ and $\mathrm{q}$, and 56 scans over $\mathrm{z}$ and $\mathrm{y}$.

Archiving the GeSE spectrum for each step allows a refined normalization of the transmission spectrometer counts by fitting the spectral $\mathrm{K} \alpha$ feature and, thus, taking into account the background due to Bremsstrahlung as seen in Figure 7.6. Normalizing with a hardware set energy gate as in measurement $S E 28$ includes background counts. However, the signal to noise ratio of the $\mathrm{K} \alpha$ peak is about 12 to 1 , and the fluctuations in the background level are below $15 \%$ throughout the total data 


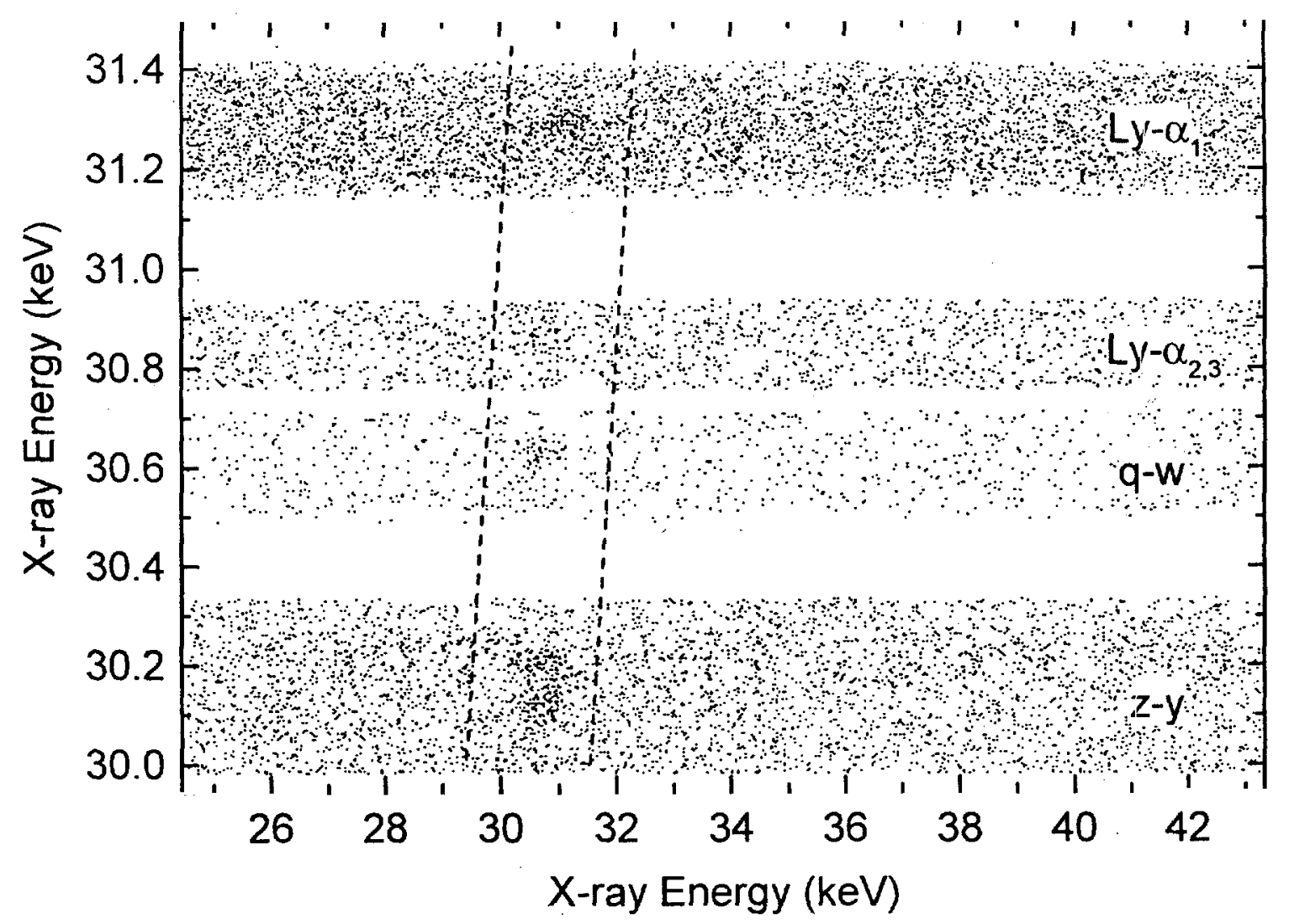

Figure 7.4: Event-mode type spectrum of the xenon $\mathrm{K} \alpha$ emission obtained with the transmission-type crystal spectrometer. This three-parameter plot displays the germanium detector (GeX) spectrum along the abscissa as function of the crystal position along the ordinate. The density of dots (and their grey shades) reflect the number of counts at each position. The dashed lines visualize the sloped gates which were set for obtaining a lineout. The labels $\mathrm{Ly}-\alpha_{1}, \mathrm{Ly}-\alpha_{2,3}, \mathrm{q}-\mathrm{w}$, and $\mathrm{z}-\mathrm{y}$ denote the main transitions covered by the different scans.

acquisition time. Therefore, the difference between the two different ways of normalization, although noticeable, is not significant with respect to the uncertainty due to the low number of counts collected with the transmission spectrometer. In particular, the line intensity ratios between the transition $y$ and $z$ are $0.73(12)$ and 0.76(17) for the measurements SE28 and SE37, respectively. Comparing transitions observed in different spectral regions, e.g., $y$ and $w$, yields intensity ratios of $0.50(8)$ for measurement SE28 and 0.62(14)-for measurement SE37. The transmission-type 


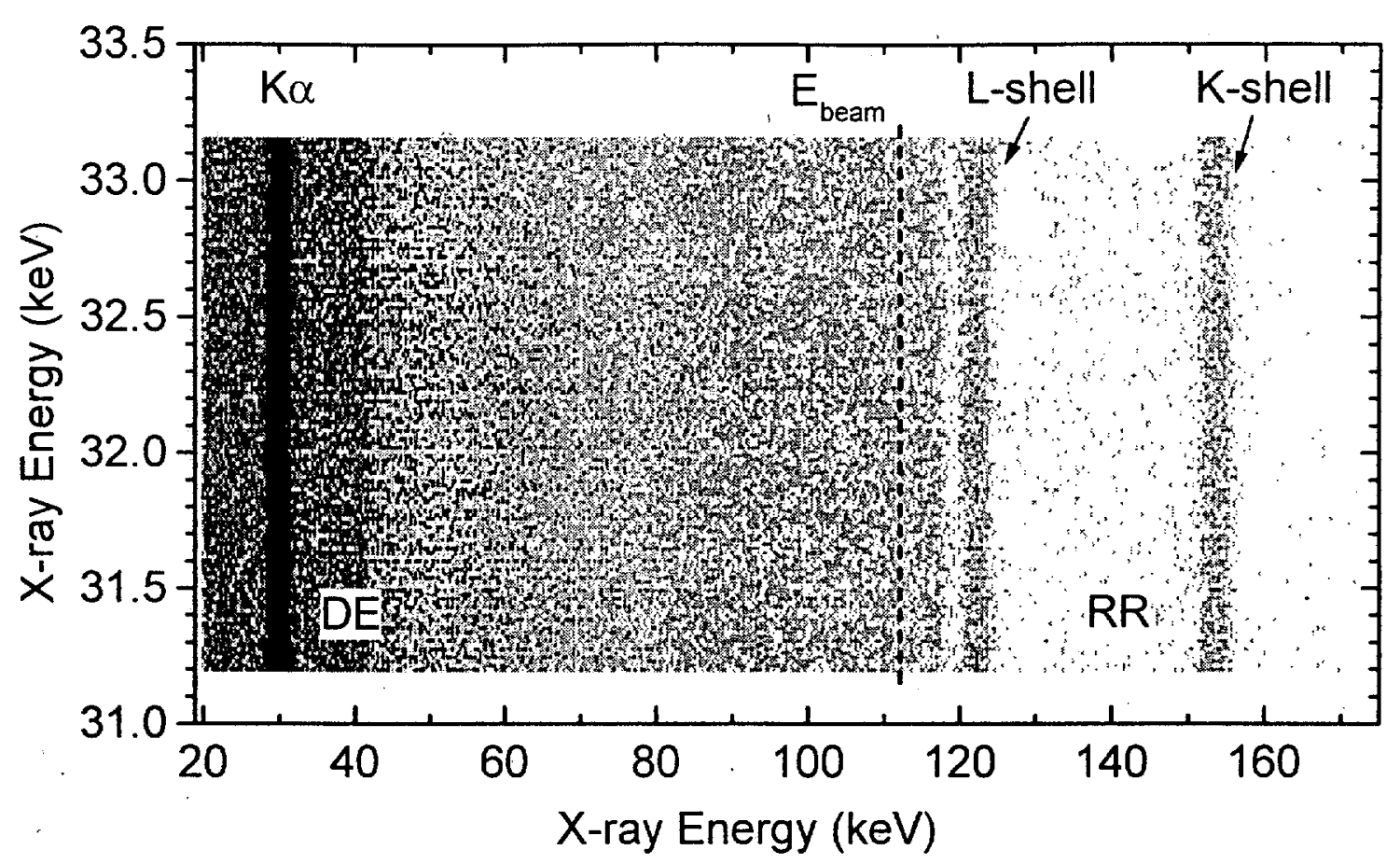

Figure 7.5: Event-mode type spectrum of the $x$-ray emission of SuperEBIT measured with the germanium detector GeSE (see Figure 7.1). This three-parameter plot displays the germanium detector spectrum along the abscissa as function of the crystal position along the ordinate. The density of dots reflect the number of counts at each position. The spectrum represents a small fraction of all the scans over the Ly $-\alpha_{1}$ spectral region.

spectra are lineouts projected onto the ordinate, i.e, the high-resolution or crystal position axis, of the three-parameter plot: The background is reduced by setting gates around the $\mathrm{K} \alpha$ lines prior to the lineout procedure. Several different lineouts were taken and separately analyzed. No significant change in the result, i.e., position of the line centers and area of the line, has been observed within the statistical uncertainties. Figure 7.7 shows a comparison of two different lineouts, i.e, a lineout with sloped gates (see Figure 7.4) and a lineout achieved by setting a vertical gate around the xenon Ka transitions. Since the observed transitions appear along the line of equal $x$-ray energy with respect to both axis, the lineout obtained with the sloped gate, i.e., parallel to this line of equal.x-ray energy, was used as the "final" 


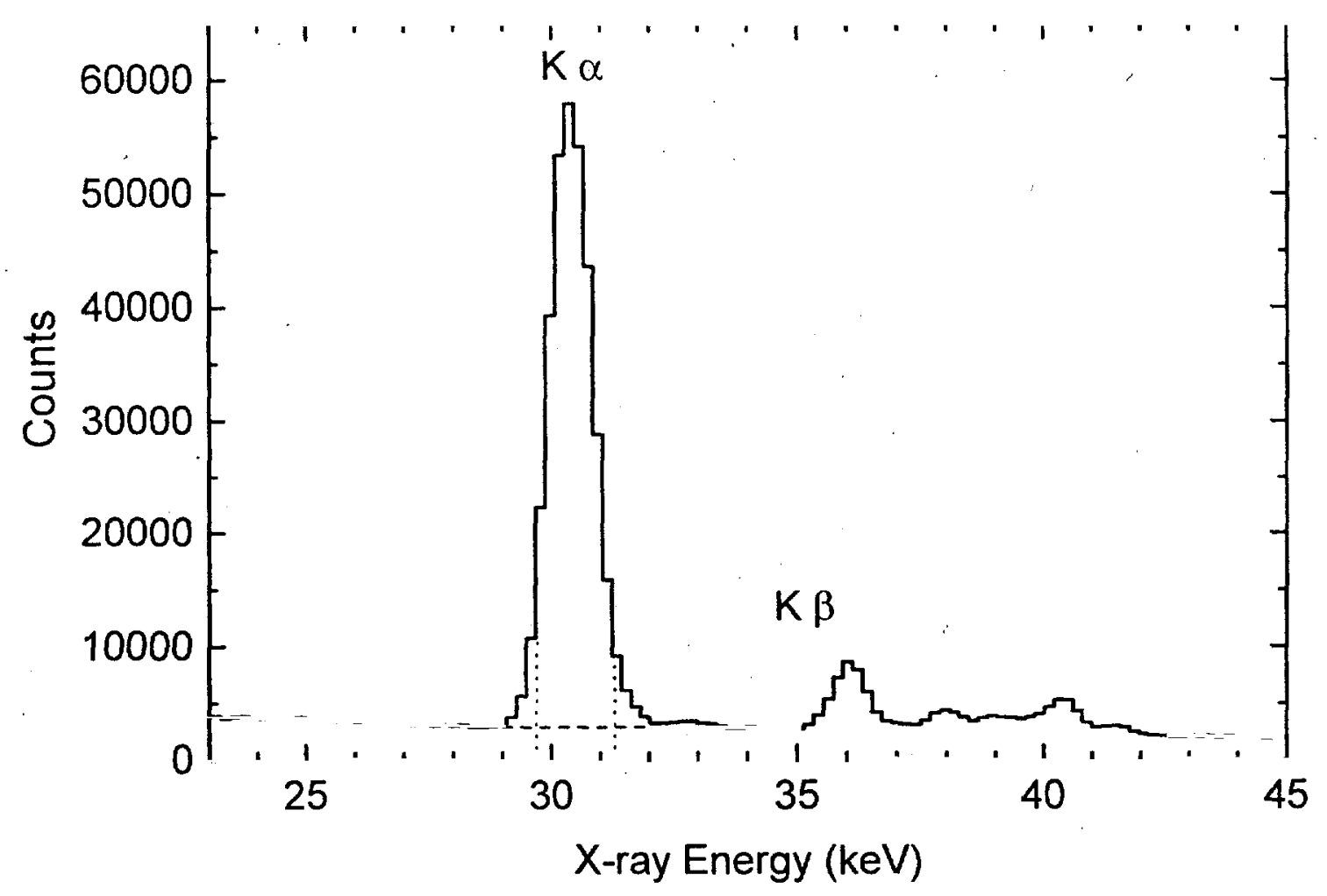

Figure 7.6: Example of a xenon. $\mathrm{K} \alpha$ emission spectrum measured with the GeSE germanium detector. The normalization of the high-resolution spectra measured with the transmission spectrometer is based on the number of photons detected by the GeSE detector within the K $\alpha$ energy interval marked by the vertical lines. For the normalization of the lineout spectra obtained from measurement $S E 37$ the $\mathrm{K} \alpha$ feature was fitted and, thus, the whole line intensity without background counts (dashed line) was taken into account.

high-resolution xenon spectrum, shown in Figure 7.8. The spectrum is normalized with respect to the overall $\mathrm{K} \alpha$ emission observed with the germanium detector GeSE (Figure 7.1). The xenon $\mathrm{K} \alpha$ spectrum measured with the germanium detector $\mathrm{Ge} \alpha_{1}$, which has a higher resolving power than GeSE, was used as an additional reference for the correctness of the normalization procedure (see also Section 7.1.3.2) below).

The efficiency of the transmission spectrometer is a crucial parameter for estimating the feasibility of its applications. The benefit of the gain in resolution is counteracted by the decrease of efficiency, which is defined by the ratio of the counted x-ray 


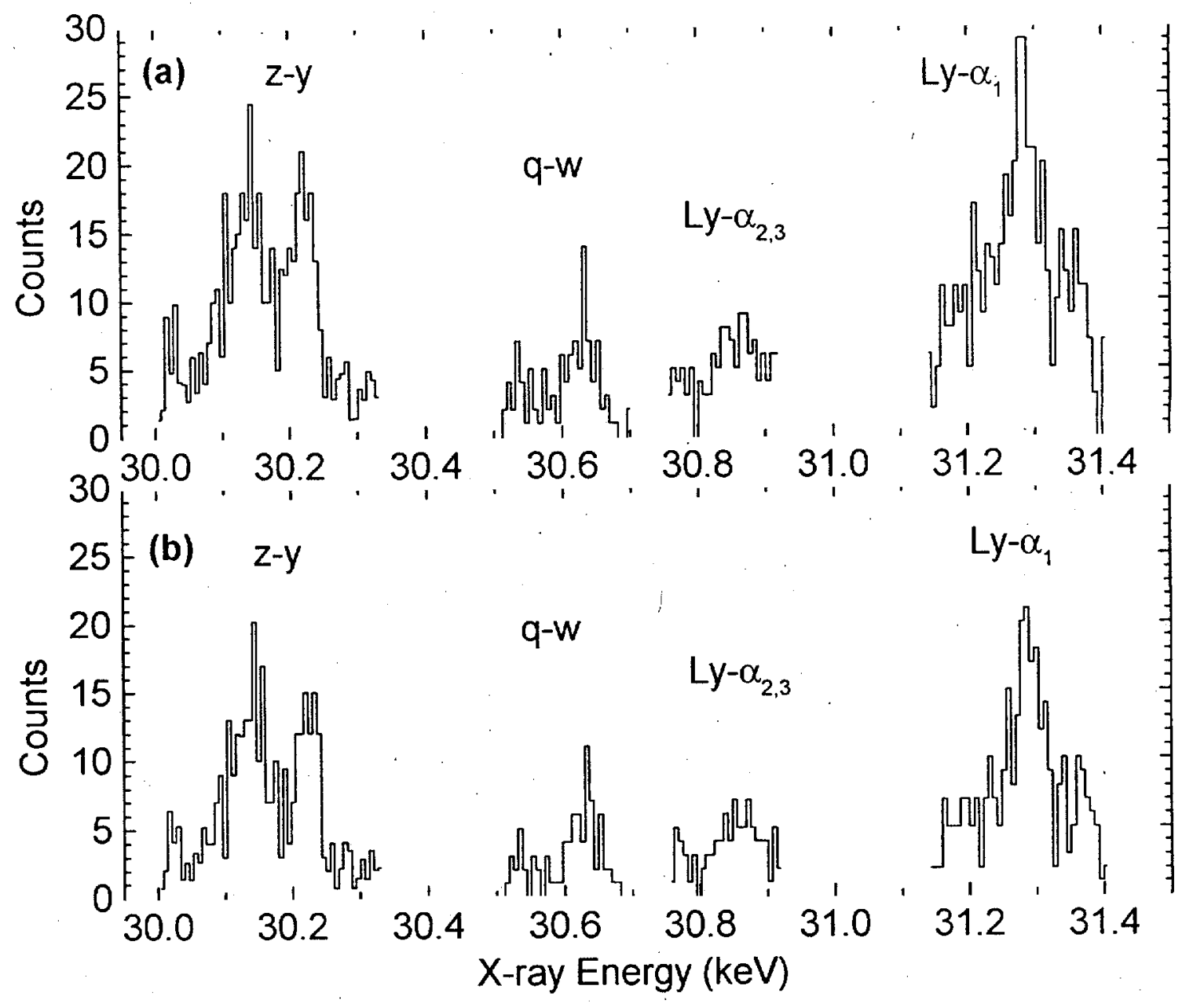

Figure 7.7: Comparison of different lineouts from the three dimensional highresolution spectrum shown in Figure 7.4. Spectrum (a) is the result obtained by setting vertical gates, i.e., a fixed energy interval independent of the crystal position. Applying a gate with a slope which is defined by the line of equal x-ray energy with respect to both axis yields the lineout shown in (b). Using the sloped gate allows to optimize the signal to noise ratio.

photons to the total number of $\mathrm{x}$-rays emitted by SuperEBIT into the transmission spectrometer's solid angle. Thus, determination of the efficiency requires:

- measuring the count rates achieved with the transmission spectrometer for the various transitions;

- estimating the $\mathrm{K} \alpha \mathrm{x}$-ray flux emitted by the highly charged xenon ions using 


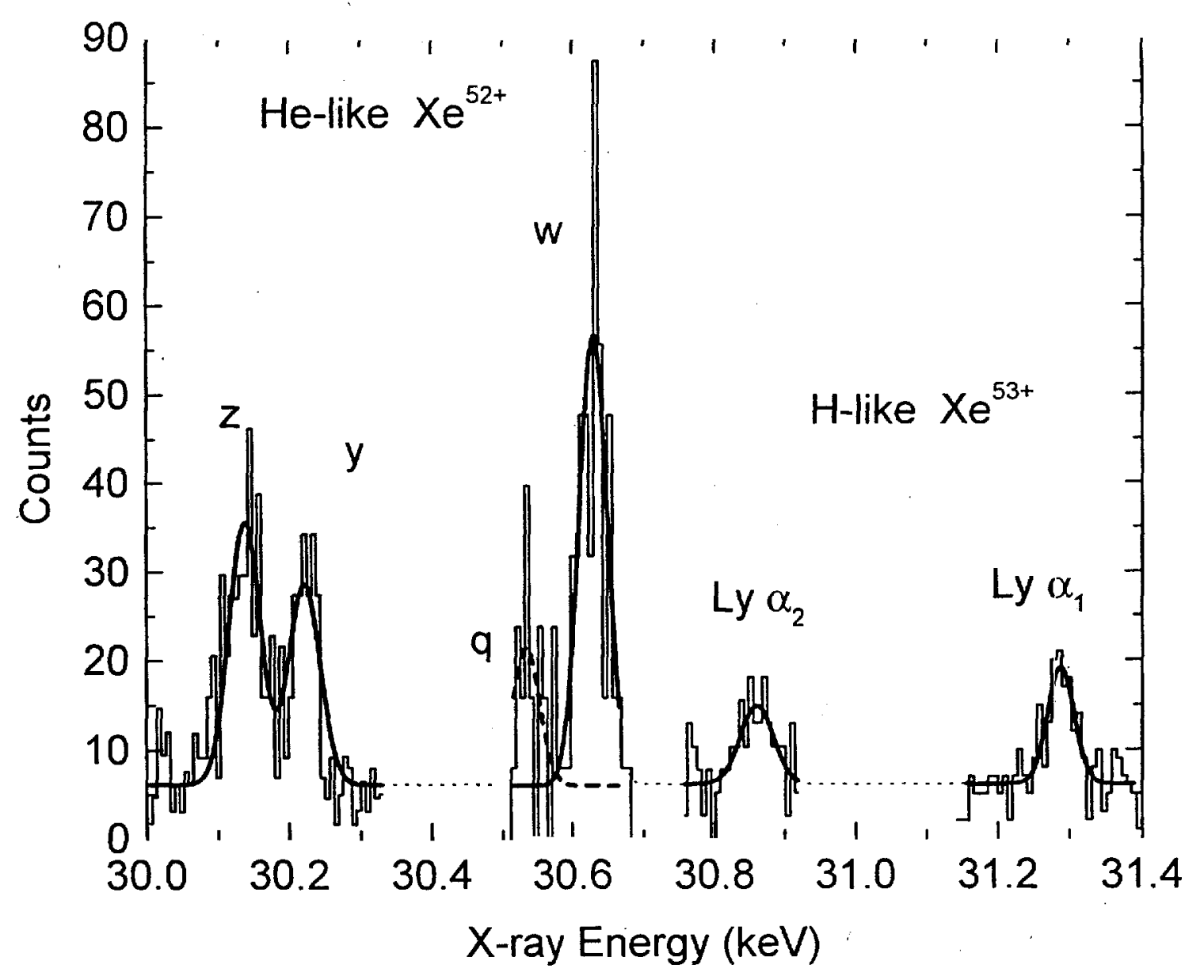

Figure 7.8: Spectrum of the K $\alpha$ transitions of hydrogenlike $\mathrm{Xe}^{53+}$ and heliumlike $\mathrm{Xe}^{52+}$. The spectrum shows the hydrogenlike $2 p_{3 / 2} \rightarrow 1 s_{1 / 2}$ (Ly- $\left.\alpha_{1}\right)$ transition, and a blend of the $2 p_{1 / 2} \rightarrow 1 s_{1 / 2}$ and $2 s_{1 / 2} \rightarrow 1 s_{1 / 2}\left(\mathrm{~L}_{y}-\alpha_{2,3}\right)$ transitions, and the heliumlike $1 s 2 p^{1} P_{1} \rightarrow 1 s^{2} S_{0}(\mathrm{w}), 1 s 2 p^{3} P_{1} \rightarrow 1 s^{21} S_{0}(\mathrm{y})$, and $1 s 2 s^{3} S_{1} \rightarrow 1 s^{2}{ }^{1} S_{0}(\mathrm{z})$ transitions. The dashed line indicates the lithiumlike $1 s 2 s 2 p^{2} P_{3 / 2} \rightarrow 1 s^{2} 2 s^{2} S_{1 / 2}$ (q) innershell transition. Each channel represents the number of detected photons accumulated over three steps, i.e., three consecutive crystal positions. The dispersion is about $6.6 \mathrm{eV}$ per channel. A resolving power of $930 \pm 110$ was inferred from the fitted line width of $L y-\alpha_{1}$ and $w$. The total data acquisition time was 122 hours.

the count rate, efficiency, and setup geometry of the GeSE and Ge $\alpha_{1}$ germanium detectors.

Since the transmission spectrometer is operated in monochromator mode and, thus, 
the number of counts obtained per step strongly depends on the crystal position, the average value while scanning over the FWHM interval of the spectral line is used to represent the count rate. A compilation of the line-specific count rates for both measurements, SE28 and SE 37, is given in Table 7.5, which also includes the overall SuperEBIT run time, the total measuring time, and the actual data acquisition time.

Table 7.5: SuperEBIT runtime, total measuring time, actual data acquisition time, and count rates for the various transition for both measurements, i.e., SE28 and SE37. The count rates are based on the average number of photons detected when scanning over the FWHM of the transitions (see Table 7.2 for spectroscopic notation). The count rate for the background (bkgd) is independent of the crystal position. Using these count rate values for estimating the time required for archiving a certain number of counts in the transition of choice, the additional time necessary for scanning the neighboring background has to be taken into account.

\begin{tabular}{rcc}
\hline \hline & SE28 & SE37 \\
\hline SuperEBIT runtime (hr) & $\approx 864$ & $\approx 440$ \\
measuring time (hr) & $\approx 323$ & $\approx 168$ \\
data acquisition time (hr) & $\approx 250$ & $\approx 122$ \\
\hline Count rates (counts per hour) & \\
Ly- $\alpha_{1}$ & $2.00 \pm 0.26$ & $9.8 \pm 1.4$ \\
Ly- $\alpha_{2,3}$ & - & $8.9 \pm 3.2$ \\
$\mathrm{w}$ & $2.97 \pm 0.25$ & $42.8 \pm 6.2$ \\
$\mathrm{q}$ & - & $13.4 \pm 5.4$ \\
$\mathrm{y}$ & $2.07 \pm 0.26$ & $26.6 \pm 4.7$ \\
$\mathrm{z}$ & $2.69 \pm 0.30$ & $35.0 \pm 5.0$ \\
bkgd & $1.36 \pm 0.06$ & $7.46 \pm 0.37$ \\
whole $\mathrm{k} \alpha$ spectrum & - & $137 \pm 11$
\end{tabular}


The five times higher background count rate in measurement SE37 is due to the five times bigger area of the germanium detector used in this measurement versus measurement SE28 (see Table 7.3). The over proportional increase of the count rates for the characteristic $\mathrm{x}$-rays emitted by heliumlike xenon ions is most likely due to the different ion abundance. With the pulsed gas injector used in measurement SE37 a much larger number of xenon atoms can be injected comparing to the continuous gas flow employed in SE28 without increasing the overall gas load on the SuperEBIT vacuum system. The higher beam current (up to $285 \mathrm{~mA}$ ) achieved in $S E 28$ is probably the reason for the high $L y-\alpha_{1}$ count rate with respect to count rates of the heliumlike transitions. The count rate values, however, depend on the line width of the transition and, thus, are not necessarily proportional to the relative intensities. The overall count rate for the xenon $\mathrm{K} \alpha$ spectral feature is obtained by adding the count rates for the observed transitions. The result of $137 \pm 11$ counts per hour is based only on measurement SE37. Note, that this is a simulated count rate in the sense that, firstly, the transitions can not be measured simultaneously with this setup, and secondly, the observation of a spectral line, actually, requires also scanning over the neighboring background, which takes additional time. Comparison of this count rate with the overall flux of the $30-\mathrm{keV} \mathrm{K} \alpha$ photons emitted by SuperEBIT into the transmission spectrometer's solid angle yields the efficiency of the spectrometer.

The SuperEBIT $\mathrm{x}$-ray flux is inferred from measurements utilizing spectroscopic equipment of known efficiency, e.g., a germanium detector. As shown in Figure 7.1 there are two germanium detectors, GeSE and Ge $\alpha_{1}$, which monitor the xenon $\mathrm{K} \alpha$ radiation emitted from SuperEBIT. The data acquisition for the Ge $\alpha_{1}$ detector was not interlocked with the crystal movement and, thus, the data acquisition time per cycle was $8.5 \mathrm{~s}(76 \%)$ for this detector versus $8.1 \mathrm{~s}(72 \%)$ for detector GeSE. The count rates obtained for that spectral feature are $(93.1 \pm 4.7) \frac{1}{\mathrm{~s}}$ with the GeSE and $(19.7 \pm 3.3) \frac{1}{\mathrm{~s}}$ with the $\mathrm{Ge} \alpha_{1}$. Taking into account the absorption of the $30-\mathrm{keV} x$ rays due to the beryllium windows of SuperEBIT and the detectors, the optical path 
of the $\mathrm{x}$ rays through air, and the inactive layer of the detectors, the efficiencies of the detectors are $96.1 . \%$ and $97.5 \%$ for GeSE and $\mathrm{Ge}_{1}$, respectively. The solid angles defined by the size of the illuminated detector area and the distance between the electron beam and the germanium crystal of the detector, equal $1.058(64) \cdot 10^{-2} \mathrm{sr}$ for GeSE and $2.202(29) \cdot 10^{-3}$ sr for Ge $\alpha_{1}$. The solid angle of the GeSE setup is, actually, limited by a $20.6-\mathrm{mm}\left(\frac{13}{16} "\right)$ circular lead aperture which is placed at a distance of $178 \mathrm{~mm}(7.0$ ") from the center. The quantities necessary for calculation of the solid angles, and the efficiencies are listed in Table 7.6. The flux per unit of solid angle is determined by,

$$
\Phi_{\Omega}=\frac{N_{\text {cnts }}}{\eta_{\text {det }} t \Omega}
$$

where $N_{\text {cnts }}$ is the number of counts, $\eta_{\text {det }}$ the detector efficiency, $t$ the counting time, and $\Omega$ the solid angle of the detection system. For the xenon $\mathrm{K} \alpha$ emission the differential x-ray flux emitted perpendicular to the electron beam is $\Phi_{\Omega}=(9170 \pm 610) \frac{1}{\mathrm{ssr}}$.

This value represents the weighted average of the results obtained from the two germanium detector measurements, see Table 7.6.

The solid angle of the transmission-type crystal spectrometer, which is determined by the detector area and its distance from the electron beam equals (1.4074 \pm 0.0021$)$. $10^{-4} \mathrm{sr}$. The xenon $\mathrm{K} \alpha$ flux emitted into such a solid angle is, thus, (1.282 \pm 0.095$) \frac{1}{\mathrm{~s}}$. This value includes already the absorption due to the beryllium windows of $\mathrm{Su}-$ perEBIT. Comparison with the count rate of $(137 \pm 11) \frac{1}{\mathrm{hr}}$ obtained with the transmission spectrometer yields an efficiency of $0.0296 \pm 0.0031$, i.e., $\approx 3 \%$. Putting the whole spectrometer into a helium atmosphere would increase the efficiency to $3.3 \%$.

\subsubsection{Electron-impact excitation cross sections}

The cross sections for the various electron-ion interactions are determined relative to the radiative recombination (RR) cross sections. Theoretical predictions for the radiative recombination cross sections by Scofield $[119]$ have shown agreement with measured values of the cross sections of the inverse process, i.e., photoionization, 
Table 7.6: Geometric quantities necessary for determination of the efficiency $\eta_{\text {det }}$ and the solid angle $\Omega$ of the germanium detectors GeSE, Ge $\alpha$, and GeX (sce Figure 7.1). The quantity $s_{B e}$ is the thickness of all SuperEBIT beryllium windows which are in the line of sight of the detector. The solid angle of the SuperEBIT access ports is limited by a $12.7-\mathrm{mm}$ circular aperture which is placed at a distance of $72.3 \mathrm{~mm}$ (2.845") from the center creating a maximum accessible solid angle of $0.02426 \mathrm{sr}$. The solid angle of the GeSE setup is limited by a 20.6 -mm lead aperture to $0.01058(64)$ sr. Using the count rates $\left(\frac{d N_{\text {cnts }}}{d t}\right)$ for the xenon $\mathrm{K} \alpha \mathrm{x}$-ray emitted by the highly charged ions, the differential flux $\left(\Phi_{\Omega}\right)$ was determined with eq.(7.1). The weighted average values of $\Phi_{\Omega}$ and $\Phi_{S E}$ are $9170 \pm 610$ and $221 \pm 15$, respectively.

\begin{tabular}{|c|c|c|c|c|}
\hline \multicolumn{2}{|l|}{ Quantity } & GeSE & $\mathrm{Ge} \alpha$ & $\mathrm{GeX}$ \\
\hline Detector area & $A_{\text {det }}\left(\mathrm{mm}^{2}\right)$ & 2043 & 201.1 & 1018 \\
\hline Depth of Ge crystal & $l_{G e}(\mathrm{~mm})$ & 20 & 10 & 13 \\
\hline Non-active Ge layer & $l_{n a}(\mu \mathrm{m})$ & 0.3 & 0.3 & 0.3 \\
\hline Detector Be window & $l_{B e}(\mu \mathrm{m})$ & 508 & 127 & 254 \\
\hline Path length through air & $l_{\text {air }}(\mathrm{mm})$ & $25(2)$ & $45(1)$ & $2432(1)$ \\
\hline SuperEBIT Be window & $s_{B e}(\mu \mathrm{m})$ & 152.4 & 152.4 & 152.4 \\
\hline Efficiency & $\eta_{\text {det }}(\%)$ & 96.06 & 97.52 & 87.17 \\
\hline Distance to beam & $d(\mathrm{~mm})$ & $230(3)$ & $302(2)$ & $2690(2)$ \\
\hline Solid angle & $\Omega\left(10^{-4} \mathrm{sr}\right)$ & $386(11)$ & $22.02(29)$ & $1.4074(21)$ \\
\hline Count rate & $\frac{d N_{\text {cnts }}}{d t}\left(\frac{1}{\mathrm{~s}}\right)$ & $93.1 \pm 4.7$ & $19.7 \pm 3.3$ & - \\
\hline Differential flux & $\Phi_{\Omega}\left(\frac{1}{\mathrm{SSI}}\right)$ & $9160 \pm 720$ & $9200 \pm 1500$ & - \\
\hline $\mathrm{X}$-ray rate through ports & $\Phi_{S E}\left(\frac{1}{\mathrm{~s}}\right)$ & $221 \pm 18$ & $222 \pm 37$ & - \\
\hline
\end{tabular}

within $3 \%[120,121]$, and, thus, represent a good reference. This uncertainty in the theoretically calculated values has to be treated as a systematic error.

Following steps are required for the determination of the electron-impact cross 
sections:

- Observation of the whole characteristic spectrum covering the direct excited (DE) transitions and the radiative recombination (RR) lines. The resolution of this spectrum has to be sufficient for inferring the charge balance, i.e., the relative ion abundance, from the RR lines. Knowledge of the detection efficiency with respect to the $R R$ and DE $x$-ray energies is essential for determination of the $\mathrm{DE}$ intensity relative to RR lines.

- High-resolution measurement of the DE spectrum which shows the composition of the DE radiation with respect to the different ion species. With the relative ion abundance and the intensity ratio of the DE spectrum to the RR spectrum, cross sections for the resolved spectral features can be assigned. Note, that crystal spectrometers are polarization sensitive instruments.

- The measured values represent differential cross sections for emission perpendicular to the electron beam. For conversion into total cross sections the polarization of the spectral features observed with the high-resolution instrument is required.

- The cross section values reflect a mixture of all processes that generate emission of the characteristic $\mathrm{x}$ rays. The electron-impact excitation cross sections are extracted using rate equations for the equilibrium conditions for each ion species and measurements or models for the level population due to cascading from high-n excited levels.

\subsubsection{Charge balance}

The RR spectrum of the highly charged xenon ions was observed with the germanium detector GeSE (see Figure 7.1). The data acquisition of measurement SE37 was set up for archiving this spectra at each crystal position which yields 3 -dimensional data arrays as shown in Figure 7.5 (page 136). Thus, the performance of the SuperEBIT 
device with respect to the charge balance was constantly monitored. The RR spectra are lineouts projected onto the abscissa. Figure 7.9 shows such a RR spectrum obtained while scanning over the Ly- $\alpha_{1}$ transition. The relative intensities together with the theoretical values of the RR cross section, listed in Table 7.7, enable the determination of a fractional charge balance. The spectral features were fitted utilizing a function that takes into account the response of the germanium detector (see Section 5.1.2 on page 73). The line positions were determined using the measured electron beam energy, i.e., $E_{\text {beam }}=112.073 \pm 0.020 \mathrm{keV}$ (see Table 7.4), and the theoretically predicted values for the electron binding energies listed in Table 7.7. For example, the $\mathrm{x}$-ray energies released by radiative electron capture onto the K-shell of bare and hydrogenlike xenon are $(153.373 \pm 0.020) \mathrm{keV}$ and $(152.344 \pm 0.020) \mathrm{keV}$, respectively. The uncertainty of $\pm 20 \mathrm{eV}$ is the uncertainty of the electron beam energy. No uncertainties are given for the predicted values of the ionization potentials listed in Table 7.7. The width of the RR lines is dominated by the detector resolution which is a only slowly varying function of the energy of the detected photon (see eq.(5.7), page 73). Therefore, the amplitudes of the two lines and one line width are the only free parameters for obtaining the fit for the RR K-shell spectral feature. The fit yields the fractional line intensities of 0.356 for the $R R$ onto the $\mathrm{K}$-shell of bare $\mathrm{Xe}^{54+}$ and 0.644 for the $\mathrm{RR}$ onto the $\mathrm{K}$-shell of hydrogenlike $\mathrm{Xe}^{53+}$. The detector efficiency with respect to the two transitions is $92.40 \%$ for $153.4-\mathrm{keV}$ x rays and $92.62 \%$ for $152.3-\mathrm{keV}$ x rays.

The ratio of the differential $R R$ cross section values for the two transitions is 2.0026 , which basically reflects the ratio of the K-shell vacancies for these ions. Thus, the ratio of the bare to the hydrogenlike ion abundance is $0.279 \pm 0.024$. For each bare $\mathrm{Xe}^{54+}$ ion in the trap there are about $3.6 \pm 0.3$ hydrogenlike $\mathrm{Xe}^{53+}$ ions. The fitted line intensities, the RR cross section values and the germanium detector efficiency completely determine the $\mathrm{RR}$ lines due to electron capture onto the $\mathrm{L}$-shell of bare and hydrogenlike xenon. Thus, only the amplitudes of the RR transitions onto the 

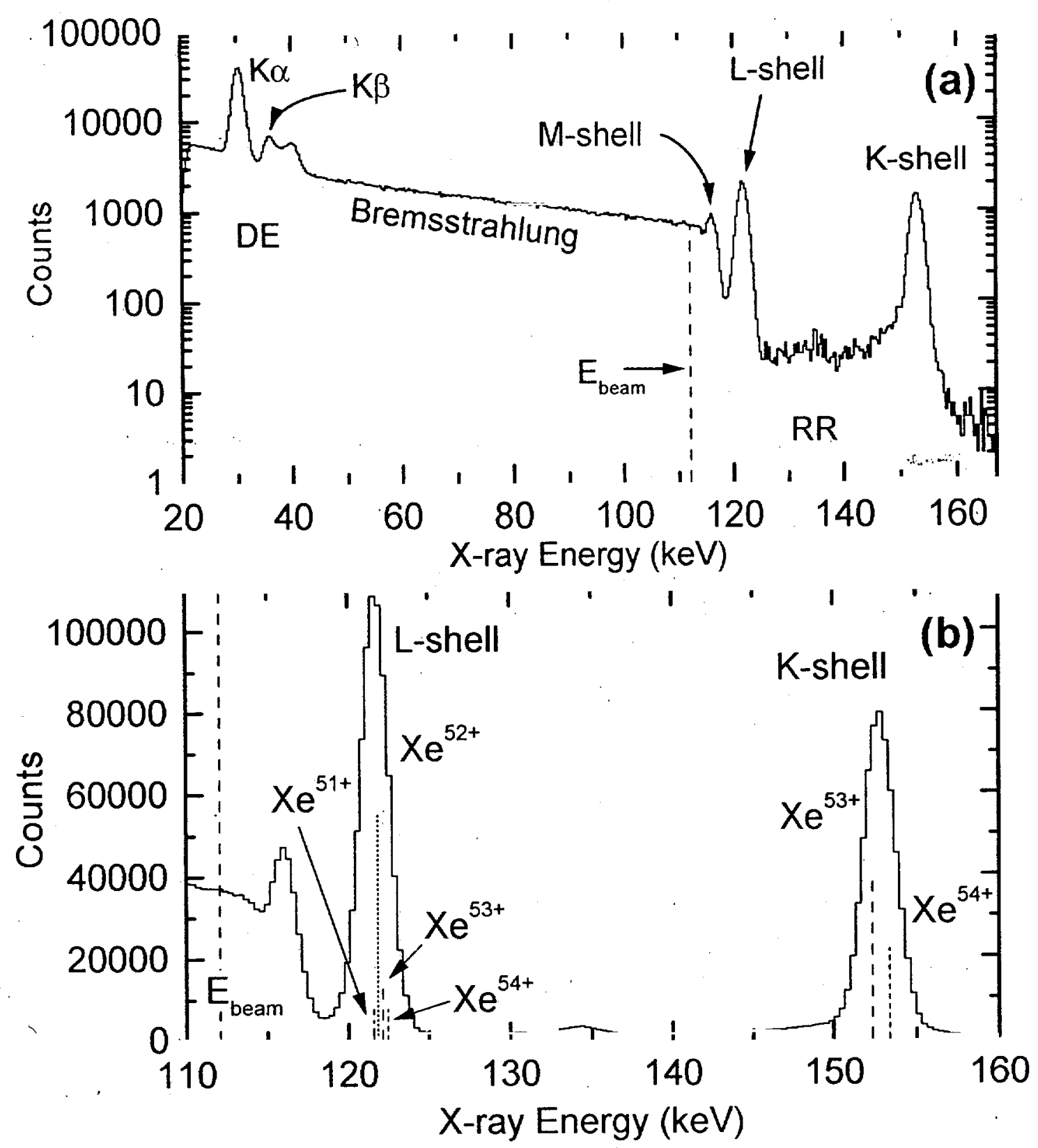

Figure 7.9: Direct excitation (DE) and radiative recombination (RR) spectra of highly charged xenon ions measured with the germanium detector GeSE (see Figure 7.1). Spectrum (a) which was taken during a one-hour measuring period shows the ratio between the $\mathrm{DE}$ and $\mathrm{RR}$ emission. The $\mathrm{RR}$ spectrum displayed in (b) was observed while scanning over the spectral regions of $L y-\alpha_{1}, L y-\alpha_{2}$, and $w$. The dashed and dotted lines mark the positions of the RR lines obtained by adding the ionization energy listed in Table 7.7 to the beam energy which was set to $112.1 \mathrm{keV}$. 
Table 7.7: Theoretically predicted RR cross section values for electron capture onto the K-shell of bare and hydrogenlike xenon, and onto the L-shell of bare, hydrogenlike, heliumlike, lithiumlike, and berylliumlike xenon. The transition energies for the RR lines are the sum of the beam energy $E_{\text {beam }}$ and the binding energy $E_{\text {bound }}$ of the subshell where the electron gets captured onto. The differential cross sections are for emission perpendicular to the electron beam, $\sigma_{\Omega, 90^{\circ}}=\left|\frac{d \sigma}{d \Omega}\right|_{90^{\circ}}$. The values are given in barn and $\frac{\text { barn }}{s r}$, respectively $\left(1\right.$ barn $\left.=10^{-24} \mathrm{~cm}^{2}\right)$.

\begin{tabular}{|c|c|c|c|c|c|c|}
\hline \multirow{3}{*}{ Ion species } & \multirow{3}{*}{ Shell } & & \multicolumn{2}{|c|}{$E_{\text {beam }}=114.1 \mathrm{keV}$} & \multicolumn{2}{|c|}{$E_{\text {beam }}=119.9 \mathrm{keV}$} \\
\hline & & $E_{\text {bound }}$ & $\sigma$ & $\sigma_{\Omega, 90^{\circ}}$ & $\sigma$ & $\sigma_{\Omega, 90^{\circ}}$ \\
\hline & & $\mathrm{keV}$ & barn & $\underset{s r}{\operatorname{barn}}$ & barn & $\frac{b a r n}{s r}$ \\
\hline \multirow{3}{*}{ bare $\mathrm{Xe}^{54+}$} & K & 41.306 & 18.256 & 1.0059 & 16.751 & 0.8897 \\
\hline & $\mathrm{L}_{\mathrm{I}, \mathrm{II}}$ & 10.432 & 2.972 & 0.1735 & 2.710 & 0.1527 \\
\hline & $\mathrm{L}_{\text {III }}$ & 10.006 & 0.524 & 0.0181 & 0.464 & 0.0155 \\
\hline \multirow{3}{*}{$\mathrm{H}$-like $\mathrm{Xe}^{53+}$} & $\mathrm{K}$ & 40.273 & 9.028 & 0.5023 & 8.284 & 0.4444 \\
\hline & $\mathrm{L}_{\mathrm{I}, \mathrm{II}}$ & 10.086 & 2.867 & 0.1689 & 2.615 & 0.1487 \\
\hline & $\mathrm{L}_{\mathrm{III}}$ & 9.628 & 0.481 & 0.0168 & 0.426 & 0.0144 \\
\hline \multirow{2}{*}{ He-like $\mathrm{Xe}^{52+}$} & $\mathrm{L}_{\mathrm{I}, \mathrm{II}}$ & 9.803 & 2.783 & 0.1645 & 2.539 & 0.1448 \\
\hline & $\mathrm{L}_{\text {III }}$ & 9.307 & 0.461 & 0.0162 & 0.409 & 0.0139 \\
\hline \multirow{2}{*}{ Li-like $\mathrm{Xe}^{51+}$} & $\mathrm{L}_{\mathrm{I}, \mathrm{II}}$ & 9.564 & 1.568 & 0.0876 & 1.426 & 0.0770 \\
\hline & $\mathrm{L}_{\text {III }}$ & 9.063 & 0.463 & 0.0162 & 0.411 & 0.0139 \\
\hline \multirow{2}{*}{ Be-like $\mathrm{Xe}^{50+}$} & $\mathrm{L}_{\mathrm{I}, \mathrm{II}}$ & 9.243 & 0.368 & 0.0117 & 0.328 & 0.0100 \\
\hline & $\mathrm{L}_{\mathrm{III}}$ & 8.865 & 0.460 & 0.0161 & 0.407 & 0.0138 \\
\hline
\end{tabular}

L-shell of the heliumlike and lithiumlike xenon ions are the variable parameters for fitting the RR L-shell feature. Adding the line representing the RR onto the L-shell of berylliumlike $\mathrm{Xe}^{50+}$ did not yield a successful fit, which shows that the amount of berylliumlike xenon is insignificant. The result of the relative ion abundances that 
were determined as described above is compiled in Table 7.8. The charge balance was determined separately for each spectral region and the values in Table 7.8 represent the weighted-average.

The absolute ion abundance, i.e., the actual number of xenon ions present in SuperEBIT, can be inferred from the flux of the characteristic RR $\mathrm{x}$ rays $\left(\Phi_{h \nu, R R}\right)$, the electron density $\left(n_{e}\right)$, the electron velocity relative to the ion $\left(v_{e}\right)$, and the RR cross section for the specific transition of interest $\left(\sigma_{R R}\right)$. In particular, the absolute abundance of ions with the charge state $q$ is,

$$
N_{q}=\frac{\Phi_{h \nu, R R}}{n_{e}\left\langle v_{e} \sigma_{R R_{q \rightarrow q-1}}\right\rangle} .
$$

Since the electron velocities are several orders of magnitude higher than the ion velocities and the electron velocity distribution is insignificant with respect to the mean velocity, $\left\langle v_{e} \sigma\right\rangle$ can be substituted with the product $v_{e} \sigma$. The flux of the characteristic $\mathrm{RR} \times \mathrm{x}$ rays is determined from the measured count rates, solid angle, and efficiency of the detector setup (see eq.(7.1). The count rates obtained with the measured line intensities are $1.64 \pm 0.14 \frac{1}{\mathrm{~s}}$ for recombination onto the $\mathrm{K}$-shell of bare $\mathrm{Xe}^{54+}$, and $2.96 \pm 0.25 \cdot \frac{1}{\mathrm{~s}}$ for recombination onto the $\mathrm{K}$-shell of hydrogenlike $\mathrm{Xe}^{53+}$. The efficiency of the GeSE detector setup is $90.77 \%$ for $153-\mathrm{keV} x$ rays, and the solid angle equals $1.058(64) \cdot 10^{-2} \mathrm{sr}$. Thus, the differential flux of the characteristic RR $\mathrm{K}$-shell $\mathrm{x}$ rays emitted perpendicular to the electron beam is $\Phi_{h \nu, R R}=171 \pm 18 \frac{1}{\mathrm{~s} \cdot \mathrm{Sr}}$ for bare $\mathrm{Xe}^{54+}$, and $\Phi_{h \nu, R R}=308 \pm 32 \frac{1}{\mathrm{ssr}}$ for hydrogenlike $\mathrm{Xe}^{53+}$. With $n_{e}=$ $(1.08 \pm 0.11) \cdot 10^{12} \frac{1}{\mathrm{~cm}^{3}}, v_{e}=(1.71535 \pm 0.00051) \cdot 10^{10} \frac{\mathrm{cm}}{\mathrm{s}}$ (see Table 7.4), and the differential RR cross sections the ion abundance in SuperEBIT according to eq.(7.2) is $9200 \pm 1300$ bare $\mathrm{Xe}^{54+}$ ions and $33200 \pm 4800$ hydrogenlike $\mathrm{Xe}^{53+}$ ions. The trap volume that contributes to the observed radiation is $1.071 \cdot 10^{-4} \frac{1}{\mathrm{~cm}^{3}}$, i.e., a cylinder of $1.27 \mathrm{~cm}$ height and about $0.01 \mathrm{~cm}$ diameter. This yields xenon ion densities of $n_{b}=(0.86 \pm 0.12) \cdot 10^{8} \frac{1}{\mathrm{~cm}^{3}}$ and $n_{H}=(3.10 \pm 0.44) \cdot 10^{8} \frac{1}{\mathrm{~cm}^{3}}$, with the indeces ${ }_{b, H}$ denoting the bare and hydrogenlike ion species. All measured fractional and absolute ion abundances for the various xenon charge states are listed in Table7.8. 
Table 7.8: Fractional and absolute ion abundances $\left(f_{n}, N\right)$ of trapped highly charged xenon ions $\left(\mathrm{Xe}^{54+\ldots 51+}\right)$. The abundances were determined using the line intensities of the RR transitions onto the $\mathrm{K}$-shell and $\mathrm{L}$-shell of the xenon ions (see Figure 7.9). The ion density values $(n)$ are based on an observed trap volume of $1.071 \cdot 10^{-4} \mathrm{~cm}^{3}$, i.e., a cylinder of $1.27 \mathrm{~cm}$ height and about $0.01 \mathrm{~cm}$ diameter.

\begin{tabular}{rccc}
\hline Ion species & $f_{N}$ & $N$ & $\begin{array}{c}n \\
10^{8} \mathrm{~cm}^{-3}\end{array}$ \\
\hline bare $\mathrm{Xe}^{54+}$ & $0.051 \pm 0.003$ & $9200 \pm 1300$ & $0.86 \pm 0.12$ \\
$\mathrm{H}$-like $\mathrm{Xe}^{53+}$ & $0.184 \pm 0.010$ & $33200 \pm 4800$ & $3.10 \pm 0.44$ \\
$\mathrm{He}-$ like $\mathrm{Xe}^{52+}$ & $0.631 \pm 0.033$ & $117000 \pm 19000$ & $11.0 \pm 1.8$ \\
Li-like $\mathrm{Xe}^{51+}$ & $0.139 \pm 0.043$ & $20800 \pm 7100$ & $1.94 \pm 0.66$ \\
\hline \hline
\end{tabular}

Combining the abundance of bare, hydrogenlike, heliumlike, and lithiumlike xenon gives a positive charge density of $\rho^{+}=(8.8 \pm 1.0) \cdot 10^{10} \frac{e}{\mathrm{~cm}^{3}}(e$ is the elementary charge) due to the trapped xenon ions. The space charge compensation factor, i.e., the ratio of positive to negative charges in the trap (see eq.(4.31) on page 40), equals $0.082 \pm 0.012$. This value represents a lower limit since the amount of the positively charged neon ions (neon was used as cooling gas) was not taken into account for this estimate. The rather small space charge compensation is not surprising because no potential difference was applied between the bottom, middle, and top drift tube electrodes in order to optimize the charge balance with respect to the highest charge states of the trapped xenon ions.

\subsubsection{Level-specific measurement of the xenon $\mathrm{K} \alpha$ spectrum}

Both, measurement SE28 and SE37 allow level-specific determination of the relative line intensities. However, only measurement SE37 was used for following investigation of the electron-impact excitation cross sections, because it includes the $\mathrm{Ly}-\alpha_{2,3}$ 
transitions.

Prior to a comparison between the intensity ratios of spectra measured with a germanium detector and spectra obtained with a crystal spectrometer a discussion of the polarization sensitivity of the Bragg diffraction is necessary. The reflectivity $R$ of a perfect crystal is given by:

$$
R=\frac{f_{\perp}}{f_{\|}}=|\cos 2 \theta|
$$

$f_{\perp}$ and $f_{\|}$are the reflectivity coefficients with respect to the orientation of the polarization of the incoming $\mathrm{x}$ rays. The subscripts ${ }_{\perp}$ and $\|$ stand for perpendicular, and parallel to the electron beam, respectively. The polarization $P$ is defined by:

$$
P=\frac{I_{\|}-I_{\perp}}{I_{\|}+I_{\perp}}
$$

where $I_{\perp}$ and $I_{\|}$are the intensities perpendicular and parallel to the electron beam. Parallel to the electron beam axis linearly polarized light has a polarization $P$ of +1 , completely perpendicular polarization obtains the value -1 . Using equations (7.3) and (7.4) the intensity adjustment due to the Bragg angle dependent reflectivity of the crystal has the form:

$$
I_{a d j}=I_{o b s} \frac{2}{f_{\|}[1+P+(1-P) R\rceil}
$$

with $I_{o b s}$ being the line intensity observed with the crystal spectrometer and $I_{\text {adj }}$ the polarization adjusted line intensity. Assuming that the reflectivity coefficient $f_{\|}$is constant over the small Bragg angle interval used in measurement SE37, i.e.; from $9.67^{\circ}$ to $10.04^{\circ}$, the adjustment factor $f_{P o l}$ can be written as

$$
\frac{I_{a d j}}{I_{o b s}}=f_{P o l}=\frac{2}{|1+P+(1-P)| \cos 2 \theta||} .
$$

With $|\cos 2 \theta| \approx 0.94$ the denominator modifies to $1.94+0.06 P$. Neglecting the change of the line intensities due to the polarization, thus, could cause a maximum deviation of $3 \%$ in the observed line area. 
The polarization of a line depends on the population of the magnetic sublevels in following way:

$$
\begin{aligned}
\mathrm{Ly}-\alpha_{1} \ldots P & =\frac{\sigma_{-3 / 2}-\sigma_{-1 / 2}-\sigma_{1 / 2}+\sigma_{3 / 2}}{\sigma_{-3 / 2}+\sigma_{-1 / 2}+\sigma_{1 / 2}+\sigma_{3 / 2}} \\
\mathrm{w}, \mathrm{y} \ldots P & =-\frac{\sigma_{-1}-2 \sigma_{0}+\sigma_{+1}}{\sigma_{-1}+2 \sigma_{0}+\sigma_{+1}} \\
\mathrm{x} \ldots P & =-\frac{\sigma_{-2}-\sigma_{-1}-\sigma_{+1}+\sigma_{+2}}{\sigma_{-2}+\sigma_{-1}+\sigma_{+1}+\sigma_{+2}} \\
\mathrm{z} \ldots P & =+\frac{\sigma_{-1}-2 \sigma_{0}+\sigma_{+1}}{\sigma_{-1}+2 \sigma_{0}+\sigma_{+1}}
\end{aligned}
$$

with $\sigma_{ \pm 1 / 2, \pm 3 / 2}$ and $\sigma_{0, \pm 1}$ being the cross sections for electron-impact excitation from the ground state into the specific magnetic sublevels $[122,123]$. Theoretical values for the population of the $n=2$ sublevels predicted by Reed utilizing the Sampson code $[124 \mid$ were used to calculate the polarization of the observed transitions according to equations (7.7), (7.8), (7.9), and (7.10). The results are shown in Table 7.9.

After adjusting the measured line intensities for the polarization dependent Bragg diffraction utilizing eq.(7.6) the high-resolution spectrum was normalized with respect to the xenon $\mathrm{K} \alpha$ emission observed with the GeSE detector (as described in Section 7.1.2). Correct determination of the line intensities is of utmost importance for the cross section measurements. Thus, besides the normalization with respect to the number of xenon $\mathrm{K} \alpha \mathrm{x}$ rays, additional verification of the validity of this normalization was established by comparing the intensity ratios between the three spectral features $(\mathrm{z}-\mathrm{y}),\left(\mathrm{q}-\mathrm{w}-\mathrm{Ly}-\alpha_{2,3}\right)$, and ( $\left.\mathrm{Ly}-\alpha_{1}\right)$, with the measurements of a third germanium detector, detector Ge $\alpha 1$ in Figure 7.1. The raw data and all intermediate steps of adjustment and normalization of the line intensities are listed in Table 7.10.

\subsubsection{Measured cross sections}

Using the fractional intensities measured with the transmission spectrometer, the spectral feature K $\alpha$ observed with the GeSE detector is split into the various components. The cross section values are determined by comparison with the RR spectrum 
Table 7.9: Theoretical predictions of the sub-level specific electron impact excitation cross sections for the $\mathrm{n}=1 \rightarrow 2$ transitions in heliumlike $\mathrm{Xe}^{52+}$ and hydrogenlike $\mathrm{Xe}^{53+}$ assuming an impact energy of $114 \mathrm{keV}$. The values were calculated by Kennedy Reed utilizing the Sampson code [124] $\left(1 \mathrm{barn}=10^{-24} \mathrm{~cm}^{2}\right)$. The sublevel are denoted by their magnetic quantum number $m_{J}$, where $J$ is the total angular momentum. The columns $f_{p o p}$ contain the fractional population density of each sublevel. The polarization $P$ is calculated according to equations (7.7), (7.8), (7.9), and (7.10). The relative intensities of the characteristic $x$-ray emission parallel and perpendicular to the electron beam was determined using eq.(7.4).

Hydrogenlike $\mathrm{Xe}^{53+}$

\begin{tabular}{|c|c|c|c|c|c|c|c|c|c|}
\hline Excited & $\sigma_{ \pm 1 / 2}$ & $\sigma_{ \pm 3 / 2}$ & & & $f_{p o p}$ & & $P$ & $I_{\|}$ & $I_{\perp}$ \\
\hline level & barn & barn & & $\pm \frac{1}{2}$ & $\pm \frac{3}{2}$ & & $\mathrm{P}$ & $\%$ & $\%$ \\
\hline $2 s_{1 / 2}$ & 1.114 & & & 0.500 & & & 0.000 & 0.500 & 0.500 \\
\hline $2 p_{1 / 2}$ & 2.156 & & & 0.500 & & & 0.000 & 0.500 & 0.500 \\
\hline $2 p_{3 / 2}$ & 2.821 & 1.307 & & 0.342 & 0.158 & & 0.367 & 0.683 & 0.317 \\
\hline \multicolumn{10}{|c|}{ Heliumlike $\mathrm{Xe}^{52+}$} \\
\hline Excited & $\sigma_{0}$ & $\sigma_{ \pm 1}$ & $\sigma_{ \pm 2}$ & & $f_{p o p}$ & & $P$ & $I_{\|}$ & $I_{\perp}$ \\
\hline level & barn & barn & barn & 0 & \pm 1 & \pm 2 & $P$ & $\%$ & $\%$ \\
\hline $1 s 2 p^{1} P_{1}$ & 9.676 & 3.883 & & 0.555 & 0.223 & & 0.427 & 0.714 & 0.286 \\
\hline $1 s 2 p^{3} P_{2}$ & 0.0616 & 0.0522 & 0.0233 & 0.290 & 0.246 & 0.110 & -0.383 & 0.308 & 0.692 \\
\hline $1 s 2 p^{3} P_{1}$ & 3.993 & 1.689 & & 0.538 & 0.231 & & 0.399 & 0.700 & 0.300 \\
\hline $1 s 2 s^{3} S_{1}$ & 0.0571 & 0.0576 & & 0.331 & 0.334 & & 0.004 & 0.502 & 0.498 \\
\hline
\end{tabular}

which was simultaneously observed with the same detector. The experimental setup is designed for measuring the radiation emitted perpendicular to the electron beam. Thus, the measured cross sections are differential cross sections. The total cross sections are inferred using the relation between the overall amount of emitted radiation 
Table 7.10: Measured line intensities (raw data), polarization adjusted intensities $I_{P, a d j}$ accounting for the polarization sensitive Bragg diffraction, and normalized intensity $I_{T r s p}$. The adjustment factor $f_{P o l}$ was calculated using eq.(7.6). The Bragg angle $\theta$ and the crystal reflectivity $R$ defined in eq.(7.3) are listed, too. The normalization factor $f_{\text {norm }}$ was inferred from the highly charged xenon Ka emission simultaneously measured with the germanium detectors GeSE and Ge $\alpha_{1}$ (see Figure 7.1).

\begin{tabular}{lcccccccc}
\hline Line & $\begin{array}{c}\text { Raw data } \\
\text { Counts }\end{array}$ & $\mathrm{P}$ & $\theta$ & $R$ & $f_{\text {Pol }}$ & $I_{P, a d j}$ & $f_{\text {norm }}$ & $\begin{array}{c}I_{\text {Trsp }} \\
\text { Counts }\end{array}$ \\
Ly $-\alpha_{1}$ & $88 \pm 13$ & 0.354 & 9.67 & 0.944 & 1.019 & $89 \pm 13$ & 1.00 & $89 \pm 13$ \\
Ly- $\alpha_{2,3}$ & $31 \pm 11$ & 0.000 & 9.80 & 0.942 & 1.030 & $32 \pm 11$ & 2.59 & $83 \pm 29$ \\
$\mathrm{w}$ & $48 \pm 7$ & 0.416 & 9.87 & 0.941 & 1.017 & $49 \pm 7$ & 8.00 & $391 \pm 54$ \\
$\mathrm{q}$ & $15 \pm 6$ & - & 9.90 & 0.941 & 1.030 & $15 \pm 6$ & 8.00 & $121 \pm 47$ \\
$\mathrm{y}$ & $85 \pm 15$ & 0.390 & 10.01 & 0.940 & 1.019 & $87 \pm 15$ & 2.80 & $243 \pm 43$ \\
$\mathrm{z}$ & $112 \pm 16$ & 0.005 & 10.04 & 0.939 & 1.031 & $115 \pm 16$ & 2.80 & $323 \pm 46$
\end{tabular}

of a dipole source $(I)$ and the intensity in perpendicular direction with respect to the dipole axis $\left(I_{90^{\circ}}\right)$ accounting for the polarization $(P)$ of the radiation [125]:

$$
I=4 \pi \frac{3-P}{3} I_{90^{\circ}} .
$$

Table 7.11 presents the result of the measured differential and inferred total cross sections. The measured values are compared to theoretical cross section values calculated by Reed $|124|$. The two sets of calculations are based on a non-relativistic, and a relativistic approach. Comparison with the measurement clearly shows the importance of including relativity. But even the fully relativistic treatment shows significant deviation from the measured values.

Before discussing the differences between measured and calculated values the mag- 
Table 7.11: Measured differential cross sections, $\sigma_{\Omega, 90^{\circ}}$, and inferred total cross sections, $\sigma_{\text {expt }}$, using eq.(7.11). The values are compared with calculations of the electron-impact cross sections utilizing the Sampson code [124], see Table 7.9. The two sets of calculations for the heliumlike transitions are based on a non-relativistic, $\sigma_{\text {non, }}$, and a relativistic approach $\sigma_{\text {rel }}$.

\begin{tabular}{|c|c|c|c|c|c|}
\hline Line & $\begin{array}{c}\sigma_{\Omega, 90^{\circ}} \\
\operatorname{barn} / \mathrm{sr}\end{array}$ & $\frac{3-P}{3}$ & $\begin{array}{l}\sigma_{\text {expt }} \\
\text { barn }\end{array}$ & $\begin{array}{l}\sigma_{n o n} \\
\text { barn }\end{array}$ & $\begin{array}{l}\sigma_{r e l} \\
\text { barn }\end{array}$ \\
\hline $\mathrm{Ly}-\alpha_{1}$ & $0.89 \pm 0.14$ & 0.882 & $9.8 \pm 1.6$ & - & 8.256 \\
\hline $\mathrm{Ly}-\alpha_{2,3}$ & $0.82 \pm 0.30$ & 1.000 & $10.4 \pm 3.7$ & - & 6.541 \\
\hline w & $1.09 \pm 0.17$ & 0.861 & $11.8 \pm 1.8$ & 21.64 & 17.45 \\
\hline y & $0.68 \pm 0.13$ & 0.870 & $7.4 \pm 1.4$ & 0.127 & 7.313 \\
\hline z & $0.90 \pm 0.15$ & 0.998 & $11.3 \pm 1.8$ & 0.123 & 0.172 \\
\hline
\end{tabular}

nitude of contributions which populate the $n=2$ levels due to processes other than direct electron-ion impact excitation of a ground state electron has to be estimated. The processes considered for in following discussion are:

- Radiative recombination;

- Charge exchange processes between neutrals and the highly charged xenon ions;

- Innershell ionization of lithiumlike xenon ions;

- Population of the $\mathrm{n}=2$ levels due to cascades after electron-impact excitation of $n>2$ levels;

A schematic diagram regarding these processes is shown in Figure 7.10 and Figure 7.13. The time evolution for the abundance of each ion species is characterized by the magnitude of the rates, i.e., the rate for radiative recombination, charge exchange, electron-impact ionization, and the rate at which the ions leave the trap. 


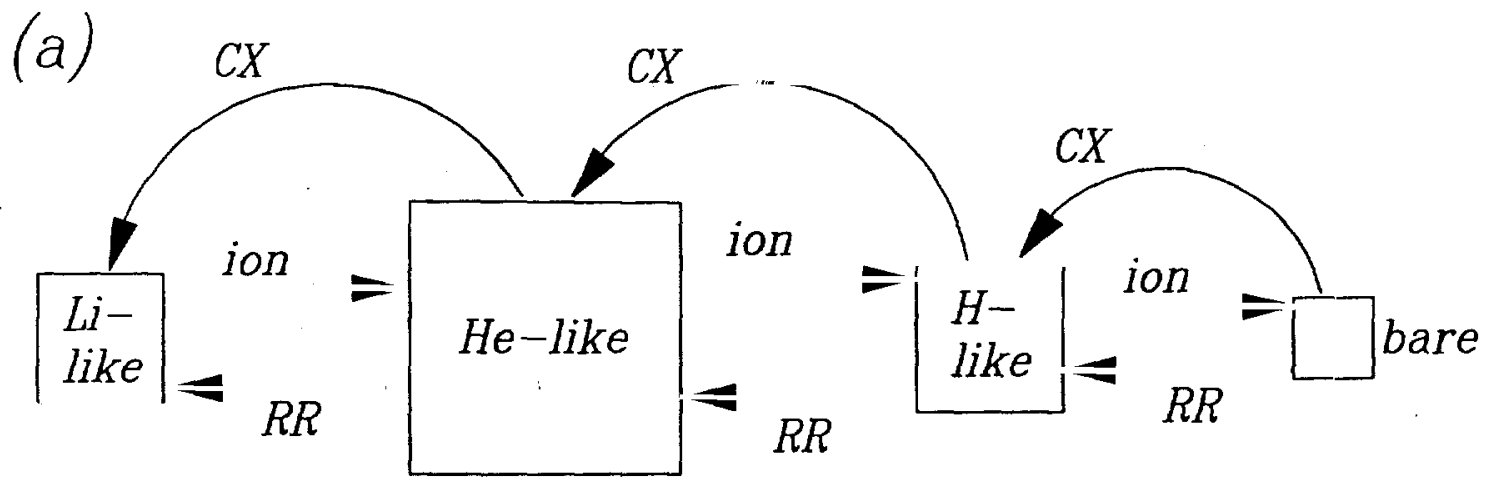

(b)

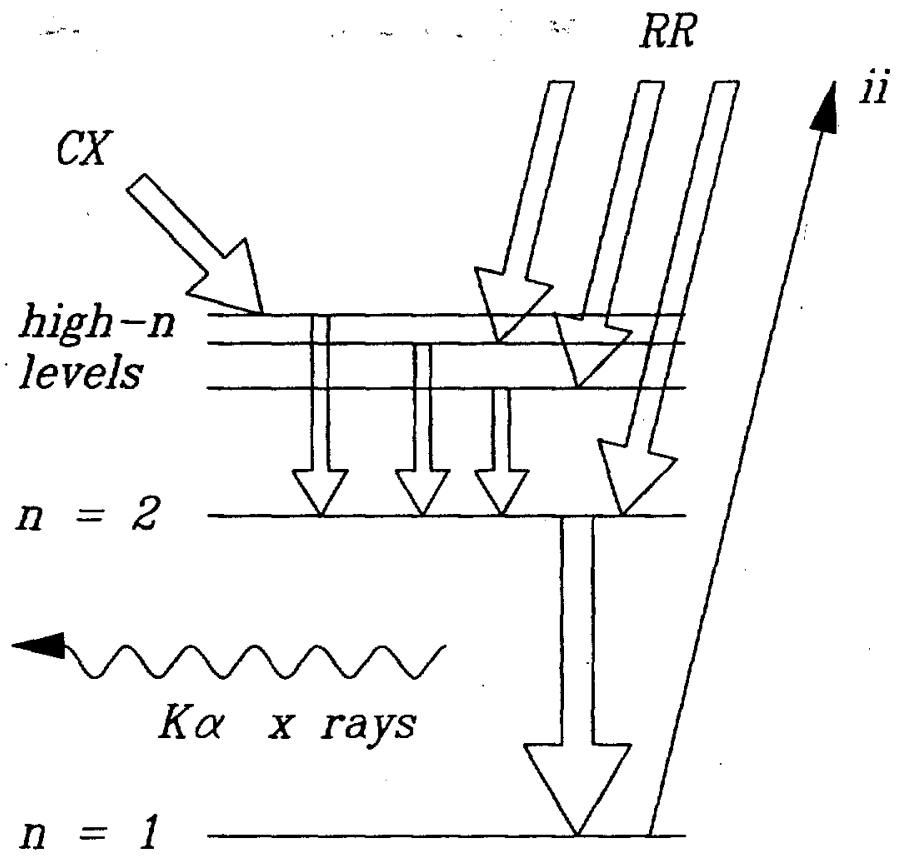

Figure 7.10: Schematic diagram of the main processes for (a) the charge balance and (b) the population of the $\mathrm{n}=2$ levels. The charge balance in SuperEBIT is in equilibrium and, thus, the contribution due to radiative recombination $(R R)$, electron-impact ionization (ion), and charge exchange $(C X)$ are balanced. The area of each box is proportional to the abundance. The processes which populate the $\mathrm{n}$ $=2$ levels other than direct excitation due to electron inpact are sketched in (b). The measured intensity of the $\mathrm{K} \alpha$ radiation is proportional to the population of the $\mathrm{n}=2$ levels. Only the innershell ionization (ii) of lithiumlike ions has to be taken into account as contributing factor to the $\mathrm{K} \alpha$ emission, in particular, to the line $\mathrm{z}$.

The rate equation for ions of the charge state $q$ is:

$$
\begin{array}{cccc}
d N_{q} & =n_{e}\left\langle v_{e} \sigma_{R R_{q+1 \rightarrow q}}\right\rangle N_{q+1} & +n_{e}\left\langle v_{e} \sigma_{i o n_{q-1 \rightarrow q}}\right\rangle N_{q-1} & +n_{0}\left\langle v_{0} \sigma_{C X_{q+1 \rightarrow q}}\right\rangle N_{q+1} \\
d t & -n_{e}\left\langle v_{e} \sigma_{R R_{q \rightarrow q-1}}\right\rangle N_{q} & -n_{e}\left\langle v_{e} \dot{\sigma}_{i o n_{q \rightarrow q-1}}\right\rangle N_{q} & -n_{0}\left\langle v_{0} \sigma_{C X_{q \rightarrow q-1}}\right\rangle N_{q} \\
- & l_{\text {trap }} N_{q} & 155 &
\end{array}
$$


$N_{q}$ is the abundance of ions with the charge $q, n_{e}$ is the electron density, $n_{o}$ the density of neutrals (atoms and/or molecules), $v_{e}$ and $v_{0}$ are the velocities of the electrons and neutrals with respect to the ions. The attributes $R R, i o n, C X$ of the cross section $\sigma$ stand for radiative recombination, electron-impact ionization and charge exchange. The factor $l_{\text {trap }} N_{q}$ accounts for the amount of ions which are escaping from the trap, or from the region of observation to be more specific. Since the cross sections depend on the impact energy and, thus, on the velocity, the velocity cross section product has to be averaged over the velocity distribution and the notation $\langle v \sigma\rangle$ is used. The velocities between the neutrals and ions $v_{0}$ are dominated by the ion velocities which show a Maxwellian distribution which is cut off at an upper velocity limit at which the kinetic energy of the ion equals the trapping potential and the ions can escape the trapping region. The electron velocities are several orders of magnitude higher than the ion velocities and the electron velocity distribution is insignificant with respect to the mean velocity. Thus, the use of a single velocity instead of a velocity distribution is justified and the product $v_{e} \sigma$ can be used instead of $\langle v \sigma\rangle$.

For such highly charged ions as lithiumlike to bare xenon, the depth of the electrostatic trapping potential experienced by these ions is about equal and the loss rate can be considered independent of the charge state. Observation of the $\mathrm{K} \alpha$ emission of highly charged xenon ions have shown that during the 8.5-s data acquisition time the count rate of the $\mathrm{K} \alpha$ photons stays almost constant. The fluctuations in the count rate are in the order of a few percent and, therefore, a decrease below about $2 \%$ would not be detectable. A $2 \%$ loss during the 8.5 -s time span means a loss rate of $\approx 0.046$ ions per second. However, all the $\mathrm{x}$-ray spectra were not recorded as a function of the data acquisition time with respect to one duty cycle and, thus, results like photon flux and ion abundance present average values which implicitly include the ion loss rate. Since the amount of ions lost is proportional to the ion abundance the loss rate does not influence the charge balance. Thus, during the period of data acquisition within each cycle the charge balance can be considered as time independent and $\frac{d N_{q}}{d t}$ equals 
zero for all charge states $q$. Solving the rate equation for equilibrium conditions also requires to take out the ion loss rate term because this quantity is not balanced by any other process since the measurement was performed utilizing a pulsed gas injector and, thus, the introduction of neutral xenon took place only during the injection period which was not part of the data acquisition interval.

The $R R$ rates are calculated using the cross section values for the recombination onto the $\mathrm{K}$ - and $\mathrm{L}$-shells from Table 7.8. The $\mathrm{RR}$ cross sections for recombination onto the $\mathrm{M}$-shell are determined form the measured RR spectra. Due to the equal amount of vacancies in the $\mathrm{M}$-shell, the $\mathrm{RR}$ cross sections are roughly the same for all trapped xenon ions. With this assumption the cross section for recombination onto the $\mathrm{M}$-shell, $\sigma_{R R, M}$, is inferred from the comparison to the total RR rate for recombination onto the $\mathrm{L}-$ shell by following relation

$$
\sigma_{R R, M}=\frac{N_{c n t s, M}}{N_{c n t s, L}} \frac{\eta_{d e t, L}}{\eta_{d e t, M}} \frac{\sum_{q} \sigma_{R R_{q \rightarrow q-1}, L} N_{q}}{\sum_{q} N_{q}}
$$

where $N_{c n t s, M}$ and $N_{c n t s, L}$ are the number of counts in the M-shell and $\mathrm{L}_{r}$-shell RR lines, $\eta_{\text {det }}$ is the detector efficiency, and $\sum_{q}$ is the sum over the different charge states $q$, i.e., lithiumlike, heliumlike, hydrogenlike, and bare xenon. The ratio of counts between the $M-$-shell $R R$ lines and the $L$-shell $R R$ lines is $0.458 \pm 0.002$. The detector setup efficiency only changes by $0.76 \%$ making the ratio $\frac{\eta_{\text {det }, L}}{\eta_{\text {det }, M}}=0.992$. Using eq.(7.13), the ion abundance listed in Table 7.8 and the $\mathrm{L}$-shell cross sections from Table 7.7 yields a $R R$ cross section of $\sigma_{R R, M}=0.142 \pm 0.006$ barn for recombination onto the M-shell of each ion species. The ionization cross sections necessary for determining the ionization rates in eq.(7.12) are calculated using the Lotz formula $[126,127]$, i.e.,

$$
\begin{aligned}
\sigma_{\text {ion }, i} & =a_{i} b_{i} \frac{E / E_{\text {ion }, i}}{E E_{\text {ion }, i}}, \\
\text { where } a_{i} & =4.5 \cdot 10^{-14} \mathrm{~cm}^{2} \mathrm{eV}^{2},
\end{aligned}
$$

and $b_{i}$ is the number of equivalent electrons in the $i$-th subshell. The charge exchange rates are defined by balancing eq.(7.12). For example, the gain of bare $\mathrm{Xe}^{54+}$ due 
to electron-impact ionization of hydrogenlike $\mathrm{Xe}^{53+}$ is balanced by the loss due to capture either of a beam electron (RR) or of a bound electron (CX) from neutral atoms like neon, which is continuously injected into the trap. With an electron density of $n_{e}=(1.08 \pm 0.11) \cdot 10^{12} \frac{1}{\mathrm{~cm}^{3}}$, and an electron velocity of $v_{e}=(1.71535 \pm 0.00051)$. $10^{10} \frac{\mathrm{cm}}{\mathrm{s}}$ (see Table 7.4), the rates, normalized to one bare ion, are $+0.644(74) \frac{1}{\mathrm{~s}}$ due to ionization of hydrogenlike xenon $\left(\sigma_{i_{0 n} \rightarrow b}=9.7\right.$ barn $)$ and $-0.429(52) \frac{1}{\mathrm{~s}}$ due to RR. Requiring equilibrium conditions the charge exchange has to yield $-0.215(90) \frac{1}{\mathrm{~s}}$. Assuming an ion temperature of about $1 \mathrm{keV}$, and a neutral gas pressure of $10^{-10} \mathrm{Torr}$, i.e., $v_{0}=3.88 \cdot 10^{6} \frac{\mathrm{cm}}{\mathrm{s}}$ and $n_{0}=3.3 \cdot 10^{6} \frac{1}{\mathrm{~cm}^{3}}$, gives a charge exchange cross section of $\sigma_{C X_{b \rightarrow H}}=1.7 \cdot 10^{-14} \mathrm{~cm}^{2}$. This is in perfect agreement with recent measurements [128]. The calculated rates are listed in Table 7.12. The ionization rates are lower limits due to the fact that the Lotz formula systematically underestimates the true ionization cross sections [129|. Thus, the values for the charge exchange recombination rates are upper limits. The charge exchange cross sections inferred from the rates are listed in Table 7.13.

The fraction of the recombination and ionization processes that populates the Lshell levels and, thus, contributes to the observed line emission is estimated using a combination of measurements and models. The population of the $\mathrm{L}_{I, I I}$ and $\mathrm{L}_{I I I}$ subshells due to radiative recombination is calculated using the RR cross sections values from Table 7.7. Assuming that the distribution within a subshell is according to the statistical weights of the levels the population of the $n=2$ levels can be determined. Table 7.14 gives an overview of the types and statistical weights of the levels within the $n=2$ subshells.

The enhancement of the $\mathrm{n}=2 \rightarrow 1$ transitions at a given population density of the $\mathrm{n}=2$ levels depends on the branching ratios of the radiative decay channels. For hydrogenlike ions the rates for spontaneous transitions within the L-shell are negligible in comparison with the spontaneous radiative decay rates of the $\mathrm{n}=2 \rightarrow 1$ transitions. Thus, only the $2 s_{1 / 2}$ level has more than one significant decay channel, 
Table 7.12: Estimated rates for radiative recombination, charge exchange, and electron-impact ionization. The rates are normalized to the presence of one bare xenon ion in the trap - and, thus, 3.58 0.30 hydrogenlike, $12.7 \pm 1.0$ heliumlike, and $2.24 \pm 0.71$ lithiumlike ions (see Table7.8). Additionally, a berylliumlike xenon abundance of $\frac{1}{5}$ the amount of the lithiumlike abundance was chosen for balancing the loss of lithiumlike ions due to $\mathrm{CX}$ and $\mathrm{RR}$ with the gain due to ionization of berylliumlike ions. This would be the maximum amount of berylliumlike xenon that is not detectable with the current resolution of the RR spectra taken with the detector GeSE. Thus, there are no uncertainties listed for rates which involve the berylliumlike species since its abundance is inferred solely from the uncertainties of the RR-spectrum fitting procedure.

\begin{tabular}{ccccc} 
ion species & & $\mathrm{RR}$ & ion & $\mathrm{CX}$ \\
& & $\frac{1}{\mathrm{~s}}$ & $\frac{1}{\mathrm{~s}}$ & $\frac{1}{\mathrm{~s}}$ \\
bare $\mathrm{Xe}^{54+}$ & gain & - & $0.644 \pm 0.074$ & - \\
& loss & $0.429 \pm 0.052$ & - & $0.215 \pm 0.090$ \\
$\mathrm{H}$-like Xe & gain & $0.429 \pm 0.052$ & $4.79 \pm 0.52$ & $0.215 \pm 0.090$ \\
& loss & $0.92 \pm 0.10$ & $0.644 \pm 0.074$ & $3.87 \pm 0.54$ \\
$\mathrm{He}$-like $\mathrm{Xe}^{52+}$ & gain & $0.92 \pm 0.10$ & $4.9 \pm 1.6$ & $3.87 \pm 0.54$ \\
& loss & $1.10 \pm 0.12$ & $4.79 \pm 0.52$ & $3.8 \pm 1.8$ \\
\hline \multirow{2}{*}{ Li-like $\mathrm{Xe}^{51+}$} & gain & $1.10 \pm 0.12$ & 1.9 & $3.8 \pm 1.8$ \\
\hline \hline
\end{tabular}

i.e., the two-photon and one-photon decay to the $1 s_{1 / 2}$ groundstate $[130,131,132]$. In hydrogenlike $\mathrm{Xe}^{53+}$ the fraction of the two-photon decay is only $23 \%$. Therefore, $88.5 \%$ of the $\mathrm{L}_{\mathrm{I}, \mathrm{II}}$ subshell population contribute to the $\mathrm{Ly}-\alpha_{2,3}$ line blend. The branching ratios for the $\mathrm{n}=2$ levels in heliumlike xenon were determined using the theoretical predictions for the spontaneous radiative decay rates by Lin et al. $\{133 \mid$ and interpolating their results for xenon $(Z=54)$. Additional calculations by 
Table 7.13: The charge exchange cross sections for the highly charged xenon ions are inferred from the charge exchange rates (see Table 7.12) assuming a neutral density of $n_{0}=3.3 \cdot 10^{6} \frac{1}{\mathrm{~cm}^{3}}$ (neutral gas pressure in SuperEBIT $\approx 10^{-10}$ Torr), and a velocity of $v_{0}=3.88 \cdot 10^{6} \frac{\mathrm{cm}}{\mathrm{s}}$ between the neutral particles and the xenon ions (ion temperature $\approx 1 \mathrm{keV}$ ).

\begin{tabular}{rc}
\hline \hline ion species & $\begin{array}{c}\mathrm{CX} \\
10^{-14} \mathrm{~cm}^{2}\end{array}$ \\
\hline bare $\mathrm{Xe}^{54+}$ & $1.68 \pm 0.79$ \\
$\mathrm{H}-$-like $\mathrm{Xe}^{53+}$ & $8.4 \pm 2.2$ \\
$\mathrm{He}-$ like Xe & $2.4 \pm 1.2$ \\
\hline \hline
\end{tabular}

Table 7.14: List of the types (LS notation) and statistical weights $\left(g_{J}=2 J+1\right)$ of the levels within the $\mathrm{n}=2$ subșhells for the hydrogenlike and heliumlike isoelectronic sequence.

\begin{tabular}{ccccc}
\hline \hline & \multicolumn{2}{c}{ Hydrogenlike } & \multicolumn{2}{c}{ Heliumlike } \\
Subshell & Level & $g_{J}$ & Level & $g_{J}$ \\
\hline \multirow{2}{*}{$\mathrm{L}_{\mathrm{I}}$} & $2 s_{1 / 2}$ & 2 & $1 s 2 s^{1} S_{0}$ & 1 \\
& & & $1 s 2 s^{3} S_{1}$ & 3 \\
\hline \multirow{2}{*}{$\mathrm{L}_{\mathrm{II}}$} & \multirow{2}{*}{$2 p_{1 / 2}$} & 2 & $1 s 2 p^{3} P_{0}$ & 1 \\
& & & $1 s 2 p^{3} P_{1}$ & 3 \\
\hline \multirow{2}{*}{$\mathrm{L}_{\mathrm{III}}$} & $2 p_{3 / 2}$ & 4 & $1 s 2 p^{1} P_{1}$ & 3 \\
& & & $1 s 2 p^{3} P_{2}$ & 5 \\
\hline \hline
\end{tabular}

Osterheld (private communication) were implemented in the determination of the branching ratios. The result: $93.4 \%$ of the $\mathrm{L}_{\text {III }}$ subshell population contribute to 
the line $\mathrm{w}\left(1 s 2 p^{1} P_{1} \rightarrow 1 s^{2}{ }^{1} S_{0}\right)$, or the line blend $\mathrm{w}-\mathrm{x}$ to be specific, the remaining $6.6 \%$ populate the $1 s 2 s^{3} S_{1}$ level; $40.9 \%$ of the $\mathrm{L}_{\text {I,II }}$ subshell population contribute to the line emission of $\mathrm{y}\left(1 s 2 p^{3} P_{1} \rightarrow 1 s^{2}{ }^{1} S_{0}\right)$ and $46.7 \%$ to $\mathrm{z}\left(1 s 2 s^{3} S_{1} \rightarrow 1 s^{2} S_{0}\right)$. A sketch visualizing the obtained branching ratios is shown in Figure 7.11. The

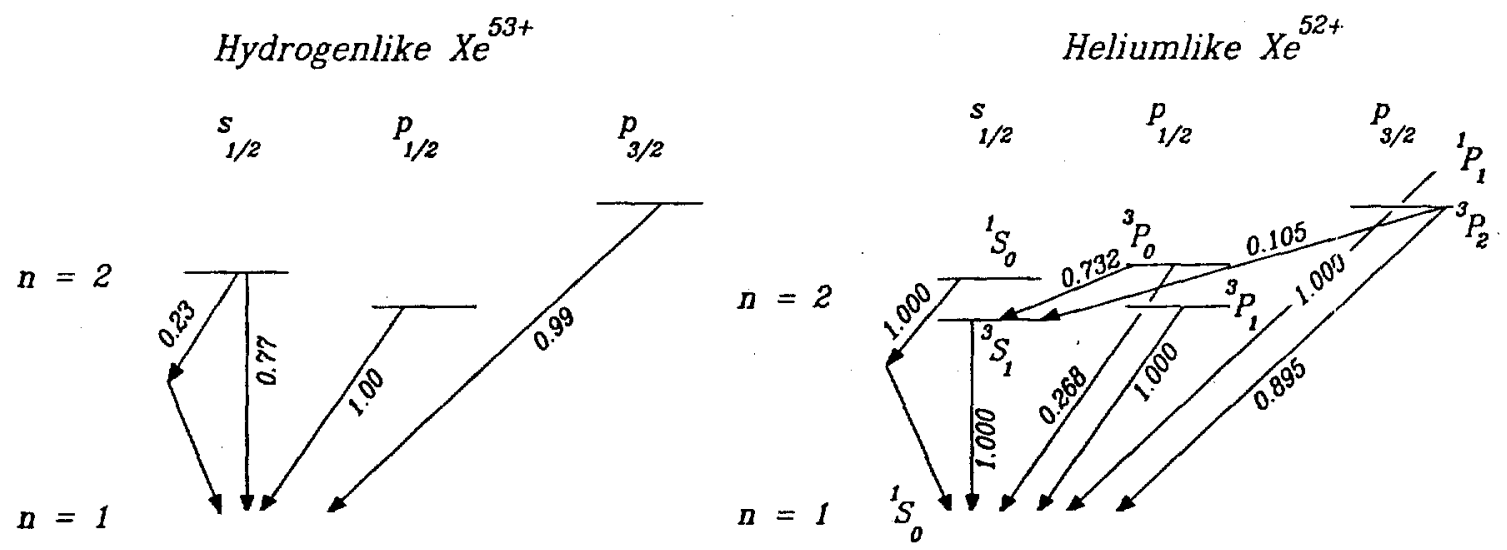

Figure 7.11: Branching ratios for the $\mathrm{n}=2 \rightarrow 1$ transitions in hydrogenlike and heliumlike xenon. The Einstein coefficients for the transitions were interpolated from theoretical predictions for several heliumlike ions [133]. The values for the $1 s 2 p^{3} P_{0} \rightarrow 1 s^{2} S_{0}$ transition and $1 s 2 s^{1} S_{0} \rightarrow \rightarrow 1 s^{2}{ }^{1} S_{0}$ two-photon transition are from Ref. $[130,131,132]$.

contribution of the $R R$ onto the $M$-shell to the population of the $n=2$ levels is neglected in this estimate. This is justifiable because for most of the transitions the branching ratios strongly favor the direct transition to the ground state. Moreover, the underestimation of the effect of RR due to neglecting the M-shell RR processes is balanced by the overestimation of the contributions due to CX processes since the CX rates calculated above are upper limits.'

The enhancement of the L-shell population of the heliumlike ions due to innershell ionization $(i i)$ of the lithiumlike species is estimated using the ionization cross sections for heliumlike xenon given by the Lotz formula in eq.(7.14), i.e., $\sigma_{i i_{L i \rightarrow H e}} \approx$ $\sigma_{i o n_{H e \rightarrow H}}=20.4$ barn. Since most of the time the ions are in the ground state the innershell ionization contributes only to the $1 s 2 s$ excited heliumlike configuration. Assuming a distribution according to the statistical weights $3 / 4$ of the heliumlike 
xenon ions that are produced by innershell ionization of lithiumlike ions have an $1 s 2 s^{3} S_{1}$ excited level and, thus, radiatively decay by emitting a $1 s 2 s^{3} S_{1} \rightarrow 1 s^{2}{ }^{1} S_{0} \mathrm{x}$ ray.

The fractional population of the $\mathrm{L}$-shell due to charge exchange recombination was measured operating SuperEBIT in the magnetic trapping mode [134]. After highly charged xenon ions were produced in SuperEBIT the electron beam was turned off, and the ions were trapped in the radial direction solely by the magnetic field. Using the pulsed gas injector a puff of xenon was injected and the $\mathrm{x}$-ray emission observed. Figure 7.12 shows the $n \geq 2 \rightarrow 1$ transitions emitted by hydrogen- and heliumlike xenon ions following charge exchange recombination with neutral xenon. With the assumption that for the hydrogenlike $\mathrm{Xe}^{53+}$ the distribution among the $\mathrm{L}_{\mathrm{I}, \mathrm{II}}$ and $\mathrm{L}_{\mathrm{III}}$ subshells is equal, which would yield an intensity ratio of 0.885 between the Ly- $\alpha_{1}$ line and the $\mathrm{Ly}-\alpha_{2,3}$ line blend, the hydrogenlike $\mathrm{n}=2 \rightarrow 1$ transitions account for roughly $1 / 3$ of all the hydrogenlike $n \geq 2 \rightarrow 1 \times$ rays. The heliumlike spectral features show that the intensity of the $n=2 \rightarrow 1$ transitions is about $2 / 3$ of the overall $n \geq 2 \rightarrow 1$ line intensities emitted by this ion species. About $40 \%$ of all the charge exchange electrons which end up in the $\mathrm{n}=2$ shell populate the $\mathrm{L}_{\mathrm{III}}$ subshell and, thus, contribute to the transition w. The rest (roughly $60 \%$ ) populate the $\mathrm{L}_{\mathrm{I}, \mathrm{II}}$ subshells enhancing the transitions $y$ and $\mathrm{z}$. The fractional line intensities of $\mathrm{y}$ and $\mathrm{z}$ are $46.7 \%$ and $53.3 \%$ respectively, according to the branching ratios determined above.

For comparison with the measured cross sections the rates are divided by the number of ions, the electron density, and the relative electron velocity. That way an electron-ion interaction equivalent cross section $(\tilde{\sigma})$ for all processes considered - including the charge exchange recombination - is calculated. Subtracting these contributions from the measured values yields cross sections which are determined by electron-impact excitation only. For the hydrogenlike transition $L y-\alpha_{1}$, for example, the measured total cross section is $\sigma_{\text {expt }}=9.8 \pm 1.6$ barn. The amount due to 


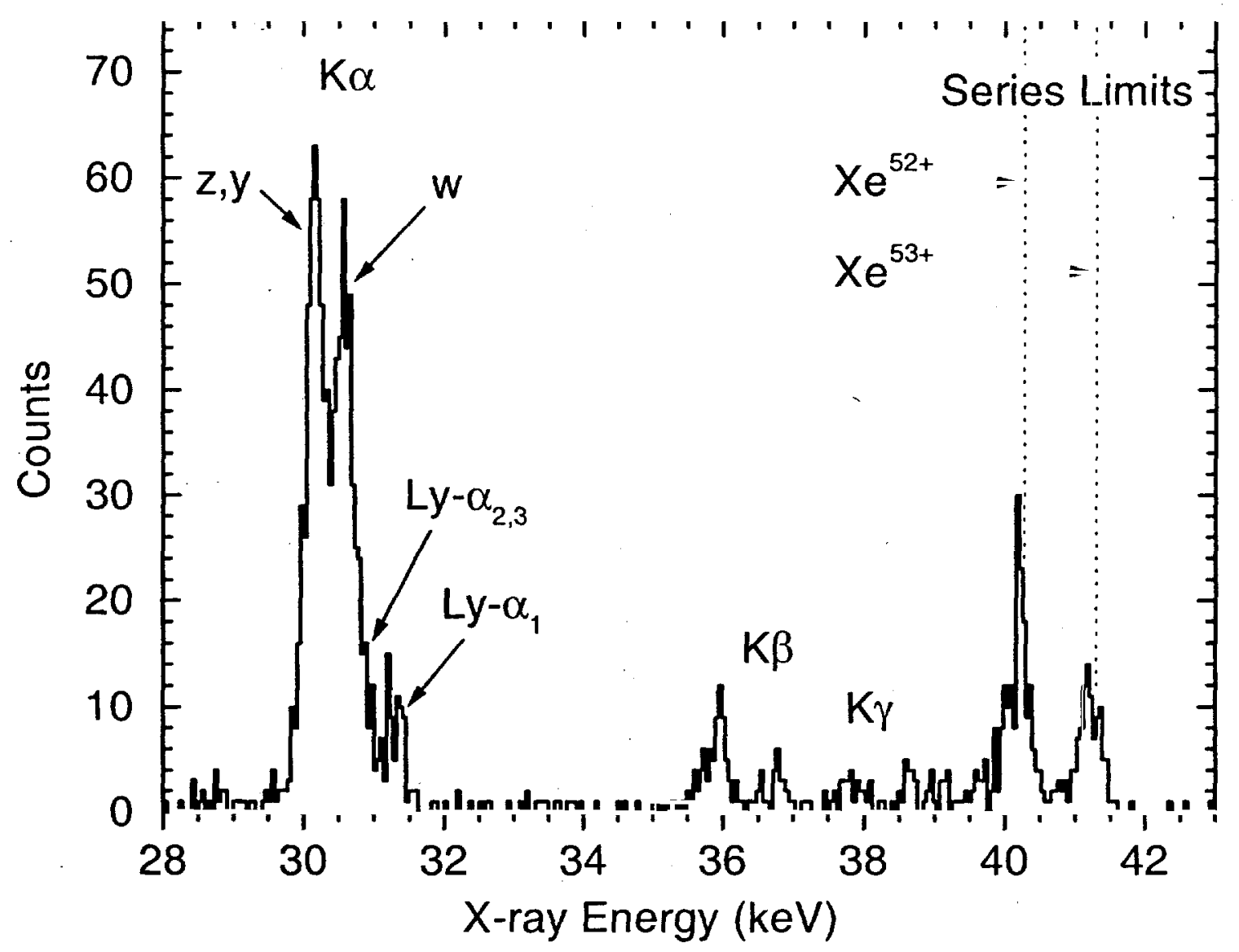

Figure 7.12: K-shell X-ray emission of helium- and hydrogenlike xenon ions after electron capture due to charge exchange with neutral xenon. SuperEBIT was operated in the magnetic trapping mode. The series limit $(n \rightarrow \infty)$ for the $n \rightarrow 1$ transition is marked with a dashed line for each ion species (values are taken from Table 7.1). The presence of counts above the series limit is due to the low resolving power of the detector. $\mathrm{K} \beta$ and $\mathrm{K} \gamma$ denote the $\mathrm{n}=3 \rightarrow 1$ and $\mathrm{n}=4 \rightarrow 1$ transitions, respectively.

RR onto the $\mathrm{L}_{\mathrm{III}}$ subshell of bare xenon equals $\tilde{\sigma}_{R R_{b-H}} \frac{N_{b}}{N_{H}}=0.146 \pm 0.13 \mathrm{barn}$, and charge exchange between bare xenon ions and neutrals account for an electronion interaction equivalent of $\tilde{\sigma}_{C X_{b \rightarrow H}}=0.57 \pm 0.26 \mathrm{barn}$. The adjusted value for the measured Ly- $\alpha_{1}$ cross section is, thus; $\sigma=9.1 \pm 1.6$ barn. This is, however, only an intermediate result, because there is one more adjustment necessary for the determination of the electron-impact excitation values. This remaining adjustment 
is the estimate of the fractional population of the $\mathrm{n}=2$ levels due to electron-impact excitation of $n>2$ levels. These contributions to the xenon $K \alpha$ spectrum were estimated using calculations by Osterheld (private communication) which included electron-impact excitation and radiative rates for all $n=2,3$, and 4 levels. The result of these calculations, which is sketched in Figure 7.13, shows that the $2 \mathrm{~s}$ levels receive a substantial fraction of their population density from upper levels. In hydrogenlike xenon $85 \%$ of the $2 s_{1 / 2}$ level is populated directly by electron-impact excitation of the ground state, i.e., $\left(1 s_{1 / 2} \rightarrow 2 s_{1 / 2}\right)$. In heliumlike xenon only $23 \%$ of the observed $1 s 2 s^{3} S_{1} \rightarrow 1 s^{21} S_{0} \mathrm{x}$ rays are due to electron-impact excitation from the ground state to this level. A compilation of the contributions due to processes other than electronimpact excitation of the $\mathrm{n}=1 \rightarrow 2$ transitions is given in Table 7.15. The electronimpact excitations are extracted by subtracting the RR, innershell ionization, and $\mathrm{CX}$ contributions from the measured values. An overview of the measured total cross sections, the experimentally determined electron-impact excitation cross sections and the theoretical predictions is given in Table 7.16 and shown in Figure 7.14.

The comparison shows that agreement between the measured and theoretically predicted values is found for the hydrogenlike transitions. The heliumlike transitions, however, significantly disagree despite the rather large uncertainties for the measured values. The disagreement is most significant for the $1 s 2 p^{1} P_{1} \rightarrow 1 s^{2}{ }^{2} S_{0}$ transition (w), where the measured and calculated values are separated by 5.3 times the 1- $\sigma$ confidence limit which presents the statistical uncertainty of the measured electron-impact excitation cross section. The cross section for the transition y, i.e., $1 s 2 p^{3} P_{1} \rightarrow 1 s^{21} S_{0}$, shows a deviation from the calculated value of 2.3 times the statistical error of the measured quantity, and for the $1 s 2 s^{3} S_{1} \rightarrow 1 s^{2}{ }^{1} S_{0}$ transition $\mathrm{z}$ the difference between measurement and theoretical prediction is 1.9 times the statistical uncertainty of the measured cross section. So far, only two measurements have been performed investigating the electron-impact cross sèctions of highly charged heliumlike ions. One measurement focused on heliumlike titanium, $\mathrm{Ti}^{20+}$, covering an impact energy range 


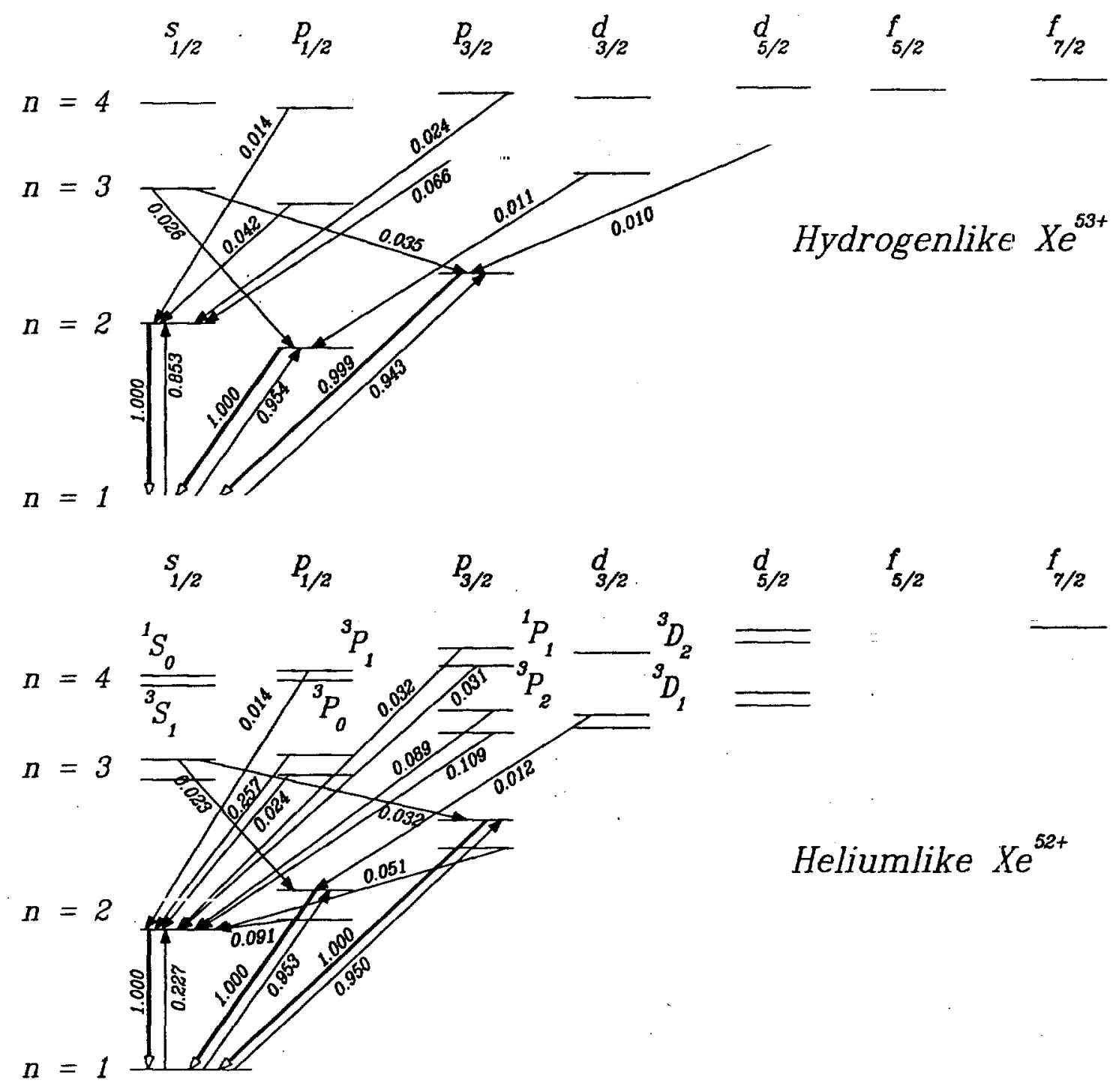

Figure 7.13: Population of the $\mathrm{n}=2$ levels in (a) $\mathrm{Xe}^{53+}$ and (b) $\mathrm{Xe}^{52+}$ due to electron-impact excitation and radiative de excitation. The model includes electronimpact excitation of all $\mathrm{n}=2,3$, and 4 levels. Only transitions with a contribution of $\geq 1 \%$ to the total population density of the excited $n=2$ levels are shown. The separation in vertical direction is according to shells, i.e., $\mathrm{K}$-shell $(\mathrm{n}=1), \mathrm{L}$-shell $(\mathrm{n}=2)$, etc., in horizontal direction according to subshells, i.e., I $\left(s_{1 / 2}\right)$, II $\left(p_{1 / 2}\right)$, III $\left(p_{3 / 2}\right)$ etc.. The heliumlike levels also include the LS notation for the levels.

from threshold up to 1.7 times threshold [135], the second measurement presents the electron-impact cross sections for heliumlike ions from $\dot{Z}=22$ to 26 , i.e., $\mathrm{Ti}^{20+}$, 
Table 7.15: Electron impact excitation cross sections for the $\mathrm{n}=1 \rightarrow 2$ transitions in heliumlike and hydrogenlike xenon. The values are extracted from the measured total cross sections after accounting for $\mathrm{RR}$, innershell ionization (ii), $\mathrm{CX}$. The contributions are given in form of an electron-ion interaction equivalent cross section (notation $\tilde{\sigma}$ ), e.g., the cross section values presenting the contribution due to CX are not the actual $\mathrm{CX}$ cross sections. The fractional population $f_{\text {pop }}$ of the $\mathrm{n}=2$ levels accounts for electron-impact excitation of all $n>2$ levels "followed" by radiative decay. The uncertainties listed combine statistical uncertainties and uncertainties in the contribution from the adjustments with respect to the $\mathrm{RR}, \mathrm{CX}$, and ii processes. A systematical uncertainties has to be "added" due to the $3 \%$ uncertainty in the theoretical values of the RR cross section which was used for the normalization of the measured electron-impact cross sections.

\begin{tabular}{|c|c|c|c|c|c|c|}
\hline line & $\begin{array}{l}\sigma_{\text {expt }} \\
\text { barn }\end{array}$ & $\begin{array}{l}\tilde{\sigma}_{R R} \\
\text { barn }\end{array}$ & $\begin{array}{l}\tilde{\sigma}_{C X} \\
\text { barn }\end{array}$ & $\begin{array}{c}\tilde{\sigma}_{i i} \\
\text { barn }\end{array}$ & $f_{p o p}$ & $\begin{array}{c}\sigma_{e e} \\
\text { barn }\end{array}$ \\
\hline $\mathrm{Ly}-\alpha_{1}$ & $9.8 \pm 1.6$ & $0.146 \pm 0.013$ & $0.57 \pm 0.26$ & - & 0.944 & $8.6 \pm 1.5$ \\
\hline $\mathrm{Ly}-\alpha_{2,3}$ & $10.4 \pm 3.7$ & $0.734 \pm 0.064$ & $0.50 \pm 0.23$ & - & 0.904 & $8.2 \pm 3.4$ \\
\hline w & $11.8 \pm 1.8$ & $0.127 \pm 0.009$ & $4.29 \pm 0.99$ & - & 0.950 & $7.0 \pm 2.0$ \\
\hline $\mathrm{y}$ & $7.4 \pm 1.4$ & $0.332 \pm 0.023$ & $3.00 \pm 0.63$ & - & 0.953 & $3.9 \pm 1.5$ \\
\hline $\mathrm{z}$ & $11.3 \pm 1.8$ & $0.388 \pm 0.027$ & $3.43 \pm 0.72$ & $2.71 \pm 0.84$ & 0.227 & $1.08 \pm 0.48$ \\
\hline
\end{tabular}

$\mathrm{V}^{21+}, \mathrm{Cr}^{22+}, \mathrm{Mn}^{23+}$, and $\mathrm{Fe}^{24+}$, near threshold for excitation of the heliumlike transitions [136]. In both measurement a slight overestimation of the electron-impact excitation cross sections for the heliumlike transitions $1 s 2 p^{1} P_{1} \rightarrow 1 s^{2}{ }^{1} S_{0}(\mathrm{w})$ and $1 s 2 s^{3} S_{1} \rightarrow 1 s^{2} S_{0}(\mathrm{z})$ was found. In a recent paper by Fontes et al. [5] the electronimpact excitation cross section calculations were refined by including the generalized Breit interaction. Fontes et al. compare the results for the collision strength of hydrogenlike and heliumlike xenon obtained by considering only Coulomb interaction and by including the generalized Breit interaction. The impact energy is close to 
Table 7.16: Comparison between the experimental and theoretical values for the electron impact excitation cross section for the $\mathrm{n}=1 \rightarrow 2$ transitions in heliumlike and hydrogenlike xenon. The experimentally achieved electron-impact excitation values $\sigma_{e e}$ are extracted from the measured total cross sections $\sigma_{\text {expt }}$ after accounting for radiative recombination, innershell ionization, and charge exchange (see Table 7.15). The uncertainties listed combine statistical uncertainties and uncertainties in the contribution from the adjustments with respect to the $R R, C X$, and ii processes. Additionally, a $3 \%$ systematical uncertainty arises from the normalization with respect to the theoretically predicted RR cross sections. The theoretically predicted values $\sigma_{\text {theo }}$ are fully relativistic calculations utilizing the Sampson code [124]. The Breit interaction was not included in this calculations.

\begin{tabular}{rccc}
\hline line & $\begin{array}{c}\sigma_{\text {expt }} \\
\text { barn }\end{array}$ & $\begin{array}{c}\sigma_{\text {ee }} \\
\text { barn }\end{array}$ & $\begin{array}{c}\sigma_{\text {theo }} \\
\text { barn }\end{array}$ \\
\hline Ly- $\alpha_{1}$ & $9.8 \pm 1.6$ & $8.6 \pm 1.5$ & 8.256 \\
Ly- $\alpha_{2,3}$ & $10.4 \pm 3.7$ & $8.2 \pm 3.4$ & 6.541 \\
$\mathrm{~W}$ & $11.8 \pm 1.8$ & $7.0 \pm 2.0$ & 17.45 \\
$\mathrm{y}$ & $7.4 \pm 1.4$ & $3.9 \pm 1.5$ & 7.313 \\
$\mathrm{z}$ & $11.3 \pm 1.8$ & $1.08 \pm 0.48$ & 0.172 \\
\hline
\end{tabular}

threshold in their calculations, in particular, the energy of the scattered electron is kept constant at $300 \mathrm{eV}$, which is, unfortunately, not achievable in any experimental setup. Including the Breit interaction has most significant impact on the $2 s \rightarrow 1 s$ transitions. For hydrogenlike xenon the electron-impact excitation cross sections for the $L y-\alpha_{2,3}$ blend are increased by $18.5 \%$, for the Ly- $\alpha_{1}$ transition, however, a decrease of $0.9 \%$ is predicted. The heliumlike transitions are similarly effected, i.e., an increase of $7.1 \%$ for $y, 7.3 \%$ for $z$, and a decrease of $7.5 \%$ for $w$. However, the theoretical values are for near-threshold conditions and, thus, can not be incorporated in the analysis of the cross sections which were measured at an electron-impact energy 


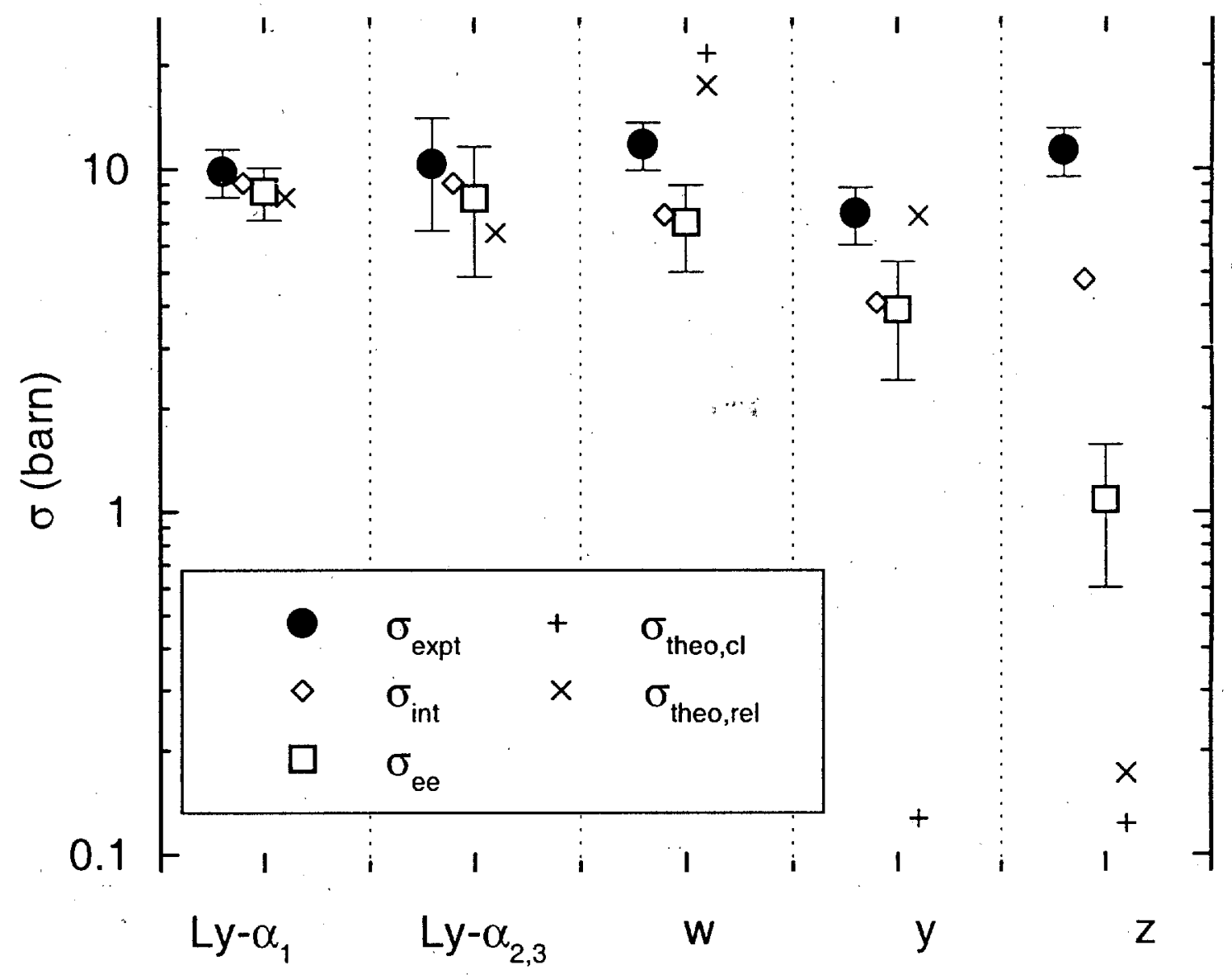

Figure 7.14: Comparison between the experimental and theoretical values for the electron impact excitation cross section for the $\mathrm{n}=1 \rightarrow 2$ transitions in heliumlike and hydrogenlike xenon. The plot shows the measured cross sections $\sigma_{\text {expt }}$, the intermediate values obtained after adjustment with respect to radiative recombination, innershell ionization, and charge exchange are denoted $\sigma_{\text {int }}$. The final electronimpact excitation values $\sigma_{e e}$ include the correction with respect to the fractional population listed in Table 7.15. The uncertainties shown are statistical uncertainties only. The theoretically predicted values have been calculated utilizing the Sampson code [124]. The $\sigma_{\text {theo,cl }}$ results (only calculated for the heliumlike transitions) do not include relativity, $\sigma_{\text {theo,rel }}$ are fully relativistic calculations.

of 3.7 times the excitation threshold. 


\subsubsection{Determination of the wavelengths}

The high precision of the crystal holder rotation mechanism allows a very accurate determination of the dispersion of the recorded high-resolution spectra. The Bragg angle difference $\Delta \theta$ between the centroids of two spectral lines $i$ and $j$ is:

$$
\begin{aligned}
\left|\Delta \theta_{i, j}\right| & =\left|\varphi_{i}-\varphi_{j}\right|= \\
& =\left|\arctan \left(\frac{x_{s, i}}{R_{l}}\right)-\arctan \left(\frac{x_{s, j}}{R_{l}}\right)\right|,
\end{aligned}
$$

with $\varphi$ being the angle of the crystal holder with respect to a arbitrarily chosen reference, $R_{l}$ the distance between the center of the rotation stage and the point of contact of the stepper-motor driven micrometer-screw, and $x_{s}$ the amount of actuator movement. The assignment of the geometric quantities is shown in Figure 7.15. The absolute values are chosen because it depends on the setup geometry whether increasing values of the crystal holder angle increase or decrease the Bragg angle. The maximum Bragg angle difference encountered during the observation of the $\mathrm{K} \alpha$ emission of the highly charged xenon ions is $0.374^{\circ}$. Therefore, the deviation due to the approximation $\varphi \approx \tan \varphi$ is below $14 \mathrm{ppm}$, much better than the accuracy achieved in following wavelengths measurement. This approximation changes the previous equation to

$$
\left|\Delta \theta_{i, j}\right|=\left|\frac{x_{s, i}-x_{s, j}}{R_{l}}\right|=\left|\frac{\left(N_{s, i}-N_{s, j}\right) \Delta x_{s t e p}}{R_{l}}\right|=\left|\Delta N_{s i, j} \varphi_{s t e p}\right| .
$$

The actuator movement $x_{s}$ is a multiple of the minimum step length $x_{s t e p}$. Thus, the' Bragg angle difference can be expressed as a multiple of the minimum angular increment $\varphi_{\text {step }}$. The number of steps $N_{s}$ between two line centroids is determined by the result of the line fitting procedure. The ball point design of the actuatorlever contact keeps the distance $R_{l}$ almost constant, i.e., the change is below $10 \mathrm{ppm}$ for a rotation of less than $0.4^{\circ}$. The minimum angular increment per step equals $\varphi_{\text {step }}=(3.569 \pm 0.005) \cdot 10^{-4} \frac{\circ}{\text { step }}$, and was measured using an optical setup.

The transmission spectrometer in its current setup is not equipped for absolute Bragg angle measurements and the determination of the wavelengths requires the 


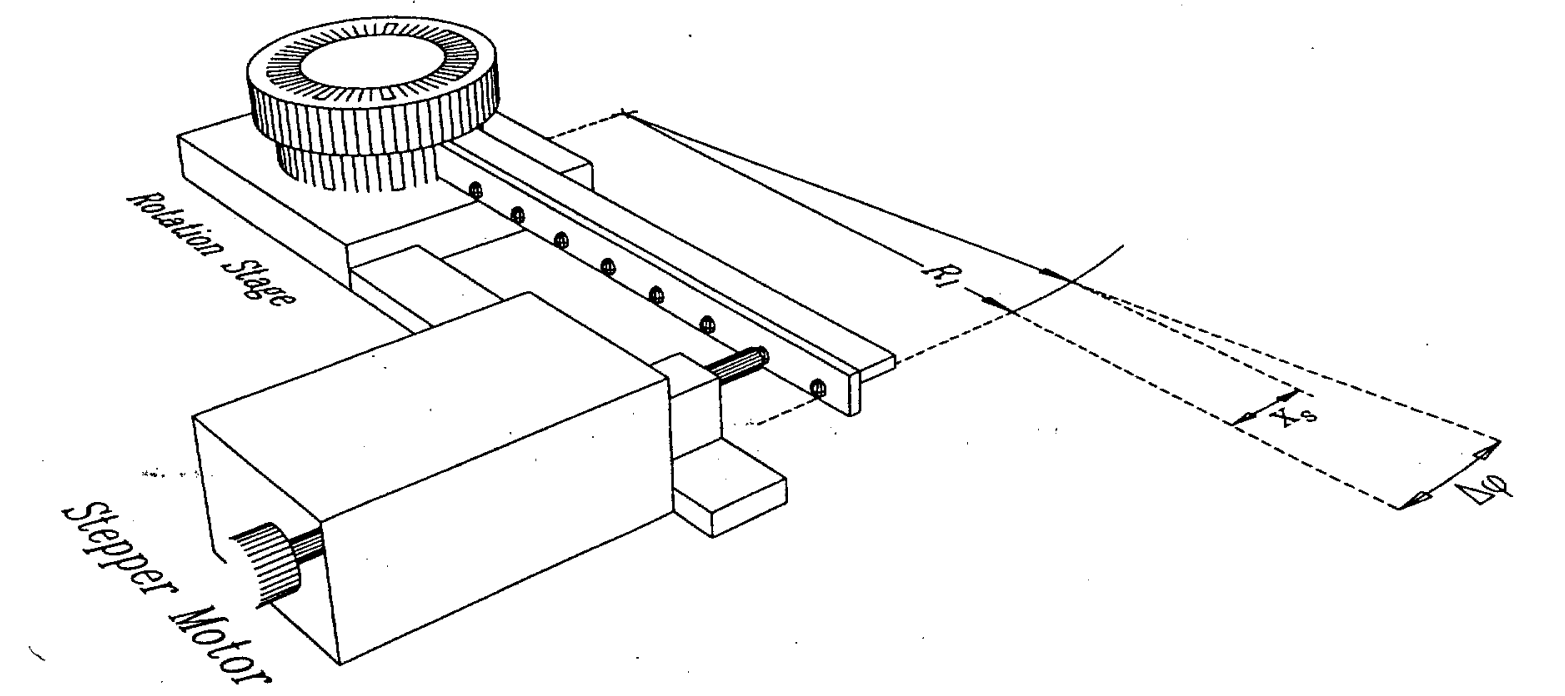

Figure 7.15: The rotation of the transmission crystal is performed by means of a stepper motor driven actuator which pushes a lever at a distance of $6 \frac{3}{8}$ " from the pivot point, i.e., the center of the rotation stage. The geometric quantities which determine the dispersion defined by this rotation mechanism are the distance between the pivot point and the point of contact of the actuator $R_{l}$, and the length of actuator movement $x_{s}$. The change in angle $\varphi$ equals the difference in Bragg angle. The ball-point design of the actuator-lever contact keeps the effective rotation arm $R_{l}$ nearly constant.

observation of at least one calibration line. Therefore, the wavelengths and transition energies were determined relative to $\mathrm{Ly}-\alpha_{1}$, since the predicted values for the transition energies in hydrogenlike systems are known to a high degree of accuracy. For the xenon Ly- $\alpha_{1}$ transition energy, for example, the uncertainty in the theoretical predictions by Johnson and Soff [27] are only $2.9 \mathrm{ppm}$, in particular, the transition energy is $31283.77(9) \mathrm{eV}$, thus, the wavelength equals $39.6321(1) \mathrm{pm}$. With this wavelength and a $2 \mathrm{~d}$-spacing of $2.3604 \AA$ for the (13̄40)-oriented Quartz crystal the Bragg angle is $9.6660^{\circ}$, according to eq. (5.9). The distance to the centroid of the neighboring $\mathrm{Ly}-\alpha_{2,3}$ blend is 380.0 steps. Using eq.(7.16) gives a Bragg angle difference of $0.136^{\circ}$ and, thus, a wavelength of $40.183 \mathrm{pm}$ for this spectral feature. The values obtained for the Bragg angles, wavelengths, and transition energies for both measurements SE28 
and SE37 are listed in Table 7.17. The accuracy is currently limited by counting statistics. In fact, an increase in the number of counts of at least a factor of 10 would be necessary to match the much lower uncertainty associated with the determination of the dispersion.

Table 7.17: Determination of the Bragg angles, wavelengths, and energies for the $\mathrm{n}$ $=2 \rightarrow 1$ transitions in hydrogenlike and heliumlike xenon. The theoretical predictions for the Ly- $\alpha_{1}$ transition energy from Johnson and Soff [27], i.e., 31283.77(9) eV, was used for calibration of the observed spectra. The uncertainty listed for the Ly$\alpha_{1}$ values are due to the uncertainty of the line centroid determination which, of course, effects the uncertainties of all the other transitions, too. The 2d-spacing

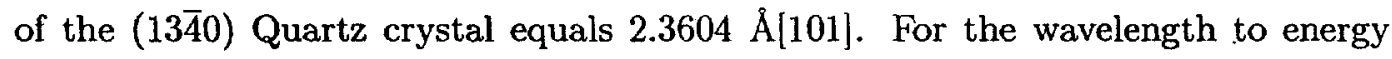
conversion the voltage-wavelength product of $V \lambda=12398.4243 \mathrm{eV} \AA$ was used.

\begin{tabular}{rcccc}
\hline \hline line & $\theta$ & $\lambda$ & $E$ & $\Delta_{\text {rel }}$ \\
& $\circ$ & $\mathrm{pm}$ & $\mathrm{eV}$ & $\mathrm{ppm}$ \\
& \multicolumn{4}{c}{$S E 28$} \\
$\mathrm{Ly}-\alpha_{1}$ & $9.6660 \pm 0.0010$ & $39.632 \pm 0.004$ & $31283.8 \pm 3.3$ & 100 \\
$\mathrm{w}$ & $9.8758 \pm 0.0013$ & $40.484 \pm 0.005$ & $30625.5 \pm 4.0$ & 130 \\
$\mathrm{y}$ & $10.0139 \pm 0.0013$ & $41.044 \pm 0.006$ & $30207.3 \pm 4.1$ & 140 \\
$\mathrm{z}$ & $10.0418 \pm 0.0012$ & $41.158 \pm 0.005$ & $30124.2 \pm 3.9$ & 130 \\
\hline $\mathrm{Ly}-\alpha_{1}$ & $9.6660 \pm 0.0007$ & $39.632 \pm 0.003$ & $31283.8 \pm 2.3$ & 70 \\
$\mathrm{Ly}-\alpha_{2,3}$ & $9.8016 \pm 0.0018$ & $40.183 \pm 0.007$ & $30855.0 \pm 5.7$ & 180 \\
$\mathrm{w}$ & $9.8761 \pm 0.0013$ & $40.485 \pm 0.005$ & $30624.5 \pm 4.0$ & 130 \\
$\mathrm{q}$ & $9.9075 \pm 0.0043$ & $40.612 \pm 0.018$ & $30529 \pm 13$ & 430 \\
$\mathrm{y}$ & $10.0113 \pm 0.0015$ & $41.034 \pm 0.006$ & $30215.1 \pm 4.5$ & 150 \\
$\mathrm{z}$ & $10.0395 \pm 0.0013$ & $41.148 \pm 0.005$ & $30131.3 \pm 3.9$ & 130 \\
\hline \hline
\end{tabular}

A comparison between measured and theoretically predicted values for the transi- 
tion energies is given in Table 7.18. For the hydrogenlike transitions Johnson and Soff [27] predict transition energies of 30856.36(9) eV for the Ly- $\alpha_{2}$ and $30863.49(9) \mathrm{eV}$ for the $\mathrm{Ly}-\alpha_{3}$ line. A line blend of $65 \% \mathrm{Ly}-\alpha_{2}$ and $35 \% \mathrm{Ly}-\alpha_{3}$ - the relative intensities are taken from a model including electron-impact excitation and radiative decay of all $\mathrm{n}<5$ levels, see Section 7.1.3.3 - would have the centroid position at $30858.9 \mathrm{eV}$ and, thus, agreement between the theoretically and experimentally achieved values exists within the $1-\sigma$ confidence limit of the measurement. For the transition energies in heliumlike systems there are several recent theoretical calculations available which are based on combining high-precision nonrelativistic variational calculations with relativistic and quantum electrodynamic corrections, also known as unified method (UM) [36], all order many body perturbation theory (MBPT) $\left[\left.37\right|^{35}\right.$, and configuration interaction (CI) calculations $[115,100 \mid$. The results utilizing the various theoretical approaches deviate from each other by less than $50 \mathrm{ppm}$. The measured values of the $\mathrm{Xe}^{52+}$ transition energies have, unfortunately, uncertainties up to $140 \mathrm{ppm}$ and, thus, do not allow to distinguish between the different theories. Despite the large uncertainties disagreement with respect to the $1-\sigma$ confidence limit has been found for all theoretical predictions for the lines $\mathrm{w}$ and $\mathrm{y}$, i.e., the $1 s 2 p^{1} P_{1} \rightarrow 1 s^{2}{ }^{1} S_{0}$ and $1 s 2 p^{3} P_{1} \rightarrow 1 s^{2}{ }^{2} S_{0}$ transitions, respectively. Figure 7.16 visualizes the comparison by plotting the relative deviation in ppm. The reference for the hydrogenlike transition energies is the result obtained by Johnson and Soff [27]. For the heliumlike transition energies the values calculated by Drake are taken as a reference [36].

Besides lowering the uncertainties of the measured values, the goal for a successful benchmark test of the theoretical predictions is calibrating the spectrum on a purely experimental basis. Such a measurement would tie the observed position of the spectral line to the primary $\mathrm{x}$-ray standard, i.e., the $\mathrm{W} \mathrm{K} \alpha_{1} \mathrm{x}$-ray emission. A possible scenario of such a calibration for the transmission crystal spectrometer measurements involves measuring well known lines emission in the 30-keV $\mathrm{x}$-ray en-

\footnotetext{
${ }^{35}$ The term "all order" in this paper refers to $(\mathrm{Z} \alpha)^{3}$ contributions.
} 
Table 7.18: Comparison between measured and theoretically predicted transition energies for the hydrogenlike and heliumlike xenon $\mathrm{K} \alpha$ lines. The measured values $E_{\text {expt }}$ are taken from Table 7.17. For the heliumlike transitions $\mathrm{w}, \mathrm{y}$, and $\mathrm{z}$ the average of both measurements SE28 and SE37 was taken. The theoretical values are from Johnson and Soff $E_{J \& S}$ [27], from Drake $E_{D}[36]$, Plante et al. $E_{P}$ [37], and Chen et al. and Cheng et al. $E_{C \& C}[115,100\}$.

\begin{tabular}{rcccc}
\hline \multicolumn{5}{c}{ Hydrogenlike transitions } \\
line & $E_{\text {expt }}$ & $E_{J \& S}$ \\
& $\mathrm{eV}$ & $\mathrm{eV}$ & \\
\hline Ly- $\alpha_{2}$ & $30855.0 \pm 5.7$ & 30856.36 & \\
Ly- $\alpha_{3}$ & \multicolumn{5}{c}{30863.49} \\
\multicolumn{5}{c}{ Heliumlike transitions } \\
\hline line & $E_{\text {expt }}$ & $E_{D}$ & $E_{P}$ & $E_{C \& C}$ \\
\hline \multicolumn{6}{c}{$\mathrm{eV}$} & $\mathrm{eV}$ & $\mathrm{eV}$ & $\mathrm{eV}$ \\
\hline $\mathrm{x}$ & $30625.0 \pm 2.8$ & 30629.28 & 30629.68 & 30630.64 \\
$\mathrm{y}$ & $30211.2 \pm 4.1$ & 30205.58 & 30205.87 & 30206.90 \\
$\mathrm{z}$ & $30127.8 \pm 3.9$ & 30128.40 & 30128.78 & 30129.79
\end{tabular}

ergy band, e.g., the $\mathrm{K} \alpha_{1,2}$ lines of cesium. Unfortunately, involving the emission of a neutral particle excludes its use during the operation of the electron bearn since immediate electron-impact ionization would shift the transition energies. Performing a successive calibration is possible by positioning a slit with a width comparable to the electron beam diameter exactly at the position of the electron beam and illuminating the slit with an $\mathrm{x}$-ray source emitting $\mathrm{Cs} \mathrm{K} \alpha$ photons, such as a ${ }^{133} \mathrm{Ba}$ radioisotope. However, the accuracy of the slit placement has to be in the order of $\pm 10 \mu \mathrm{m}$ for achieving an energy calibration with an uncertainty below $\pm 1 \mathrm{eV}$. Currently, the slit 


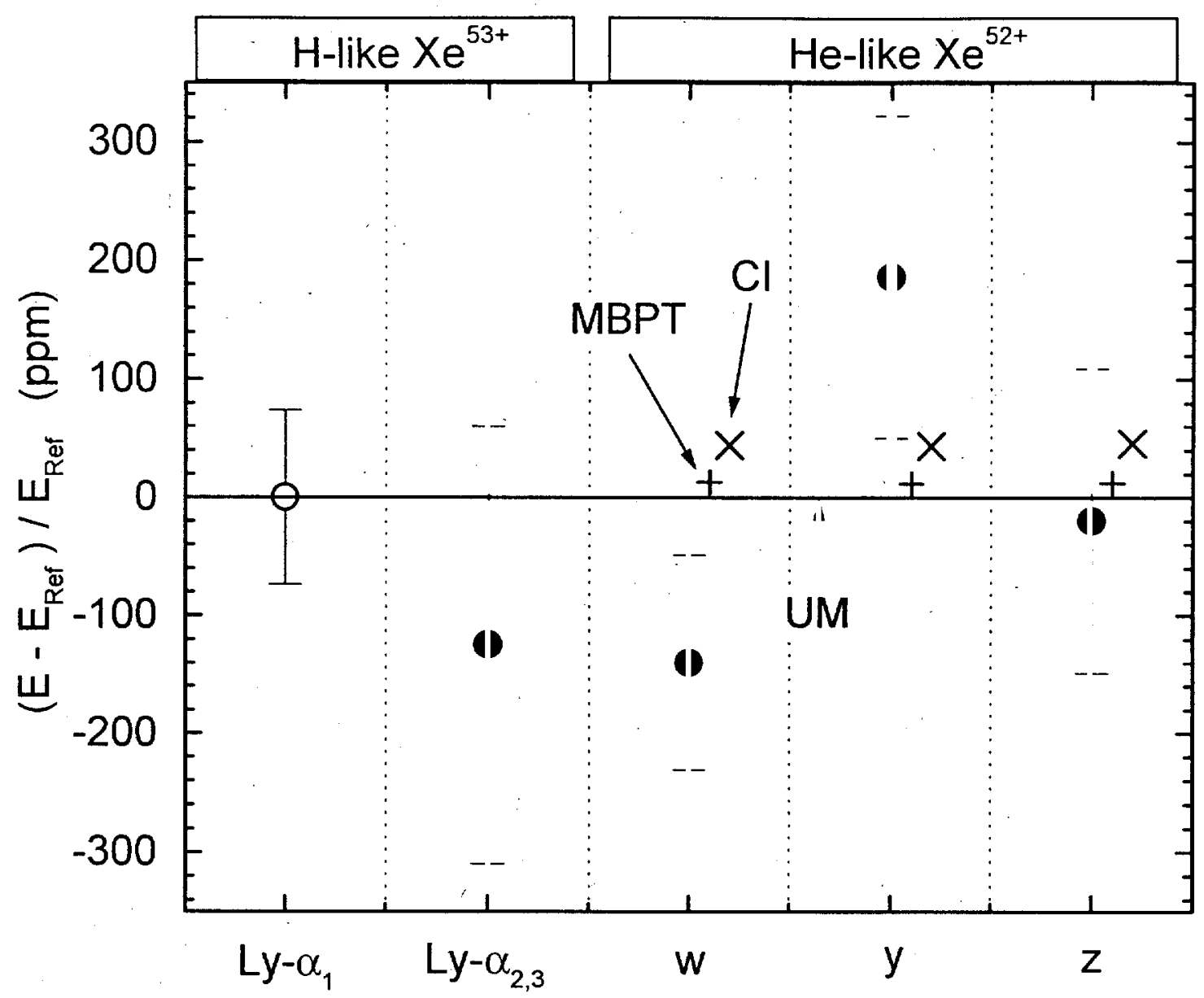

Figure 7.16: Relative deviation between measured and theoretically predicted transition energies for the hydrogenlike and heliumlike xenon $\mathrm{K} \alpha$ lines. The reference for the hydrogenlike transition energies is the result obtained by Johnson and Soff [27]. For the heliumlike transition energies the values calculated by Drake using the unified method (UM) are taken as a reference [36]. The values marked with a "+" were calculated by Plante et al. [37] summing all-orders of many-body perturbation theory (MBPT). The " $x$ " represent the values obtained by Cheng et al. and Chen et al. $[115,100]$ applying relativistic configuration interaction (CI) calculations combined with $a b$ initio QED calculations.

position is controllable only within $\pm 140 \mu \mathrm{m}$, i.e., the amount of thermal expansion of the slit mount versus drift tube mount caused by the cryogenic cooling of the SuperEBIT device. This shift introduces a systematic uncertainty of about $12 \mathrm{eV}$ for 
the measured transition energies and, thus, this method of calibration is currently not feasible. However, the need for a calibration line for the high-resolution spectra recorded with the transmission-type crystal spectrometer was the motivation for measuring the xenon $L y-\alpha_{1}$ transition energy with respect to the primary $\mathrm{x}$-ray standard utilizing state-of-the-art germanium detectors, described in Section 5.1. That way, the measured transition energies of the hydrogenlike and heliumlike lines can be tied to the measured value of the $\mathrm{Ly}-\alpha_{1}$ transition energy.

\subsection{Measurement of the $\mathrm{Ly}-\alpha_{1}$ transition energy in hydro- genlike $\mathrm{Xe}^{53+}$}

The following measurement was clearly motivated by the need of calibrating the highly resolved xenon $\mathrm{K} \alpha$ spectrum. However, a precision measurement of the $\mathrm{Ly}-\alpha_{1}$ transition energy with respect to the available $\mathrm{x}$-ray standards is an important test bed for the implementation of QED and nuclear contributions in the atomic structure calculations. The experimental goal of measuring the 1-s Lamb shift in hydrogenlike high $-Z$ ions to the sub-1-eV level promises sensitivity towards so called two-loop QED effects $\lceil 137,138,31\}$. Currently; the most precise test of QED are high-resolution measurements of the $2 p \rightarrow 2 s$ transitions in lithiumlike high- $\mathrm{Z}$ ions $[139,140,141,142\}$. However, advancements in the precision of the level energy measurements for hydrogenlike systems are still highly appreciated since those one-electron systems are the theoretically best understood configurations. Recent measurements of the hyperfine splitting of the groundstate of hydrogenlike high-Z ions have shown that the accuracy is limited by the uncertainty of nuclear parameters, such as the magnetic moment $[33,35] \mid[33,35]$. Using xenon should be, thus, an advantage since the nuclear size effects are still small in comparison with the highest- $\mathrm{Z}$ ions, like thorium and uranium, respectively. 


\subsubsection{Experimental setup and measurement}

The Ly- $\alpha_{1}$ transition energy measurement was performed parallel to the high-resolution transmission-type crystal spectrometer measurement discussed above. Two state-ofthe-art germanium detectors were used for successively observing the $\mathrm{K} \alpha \mathrm{x}$-ray radiation emitted by the highly charged xenon ions in the trap and the spectra of $\mathrm{x}$-ray sources containing the $\mathrm{K} \alpha$ emission of cesium and barium. The detectors are labeled $\mathrm{Ge} \alpha_{1}$ in the experimental setup which is shown in Figure 7.1. The two detectors are a high-purity germanium detector which will be referred to as (EGG) ${ }^{36}$, and an intrinsic germanium detector in following abbreviated as $(\mathrm{PGT})^{37}$. Both detectors had the same germanium crystal geometry, i.e., a 16-mm diameter 10-mm deep cylinder. The thickness of the beryllium windows was $127 \mu \mathrm{m}$ in both cases. The detector setup efficiency is above $97 \%$, i.e., more than $97 \%$ of the xenon $\mathrm{K} \alpha$ photons emitted into the solid angle defined by the detector are detected. This already includes the absorption due to the beryllium windows mounted in SuperEBIT and due to the air between the SuperEBIT access port and the detector beryllium window (see also Table 7.6, page 143). Due to mechanical constrains, however, the distance between SuperEBIT and the detector window was about $50 \mathrm{~mm}$ larger for the PGT detector. In particular, the optical path length through air was $95 \mathrm{~mm}$ for the PGT instead of only $45 \mathrm{~mm}$ for the EGG. Thus, the solid angle of the PGT detector setup is only $0.0016 \mathrm{sr}$ versus $0.0022 \mathrm{sr}$ for the EGG detector setup.

The nominal resolution given for $5.9-\mathrm{keV} \mathrm{x}$ rays is $195 \mathrm{eV}$ for the EGG detector and $185 \mathrm{eV}$ for the PGT detector. Using eq.(5.7) (page 73) the resolution at photon energies of about $31.3 \mathrm{keV}$, i.e., the $\mathrm{Ly}-\alpha_{1}$ transition energy, is $298 \mathrm{eV}$ and $292 \mathrm{eV}$ for the EGG and PGT, respectively. Thus, the detectors are suitable to resolve the xenon $\mathrm{Ly}-\alpha_{1}$ transition which is separated from the adjacent spectral features, the

\footnotetext{
${ }^{36}$ High-purity germanium detector with a planar crystal geometry from EG\& G ORTEC, Model No. GLP 16195/10.

${ }^{37}$ Intrinsic germanium detector with a planar crystal geometry from Princeton Gamma Tech, Model No. IGP 210185.
} 
Ly- $\alpha_{2,3}$ blend and the $1 s 2 p^{1} P_{1} \rightarrow 1 s^{2}{ }^{1} S_{0}$ transition in heliumlike xenon by more than $400 \mathrm{eV}$. Figure 7.17 shows the $\mathrm{n} \geq 2 \rightarrow 1$ transitions of highly charged xenon recorded with both detectors. The comparison between the two spectra shows that the EGG detector has, actually, the higher resolution. In particular, the measured resolution, which is inferred from the width of the recorded lines, is about $270 \mathrm{eV}$ for the EGG and $360 \mathrm{eV}$ for the PGT detector.

In two to thrce four intervals the detectors were switched between the SuperEBIT observation port and the calibration stand shown in Figure 7.1. The Ka fluorescence of cesium and barium was used for the calibration of the xenon spectra recorded at the SuperEBIT port. Thus, the calibration stand was equipped with the radioisotope ${ }^{133} \mathrm{Ba}$, which after $\mathrm{K}$-shell electron capture emits the Cs $\mathrm{K} \alpha \mathrm{x}$-rays, and the radioisotope ${ }^{241} \mathrm{Am}$, which was covered with a barium window, thus, producing the $\mathrm{Ba} \mathrm{K} \alpha$ fluorescence. The activities of the radioisotopes were $0.59 \mu \mathrm{Ci}$ for the ${ }^{133} \mathrm{Ba}$ source and $9.84 \mu \mathrm{Ci}$ for the ${ }^{241} \mathrm{Am}$ source. The calibration stand was adjusted in such a way that for the detector with the higher resolution the count rate of the Cs $\mathrm{K} \alpha$ emission was about twice the average count rate of the $\mathrm{Xe} L y-\alpha_{1}$ transition when the detector was placed at the observation port of SuperEBIT. Therefore, the count rate for the $\mathrm{Cs} K \alpha_{1}$ x rays was higher, and for the $\mathrm{Cs} K \alpha_{2} \mathrm{x}$ rays was lower than the count rate for the Xe Ly- $\alpha_{1} \mathrm{x}$ rays. Matching the count rates for measurement and calibration is important for high-precision measurements since the electrical field across the germanium crystal and, thus, the magnitude of the detected current pulse is sensitive to the amount of electron-hole pairs created by photon impact. A spectrum combining the barium, cesium, and highly charged xenon $\mathrm{K} \alpha$ emission is presented in Figure 7.18.

\subsubsection{Analysis and calibration}

The centroid position of the spectral features was determined using two different line-shape functions for the fitting process. One result was obtained by assuming a Gaussian line profile, the other by fitting a profile that accounts for the response of 


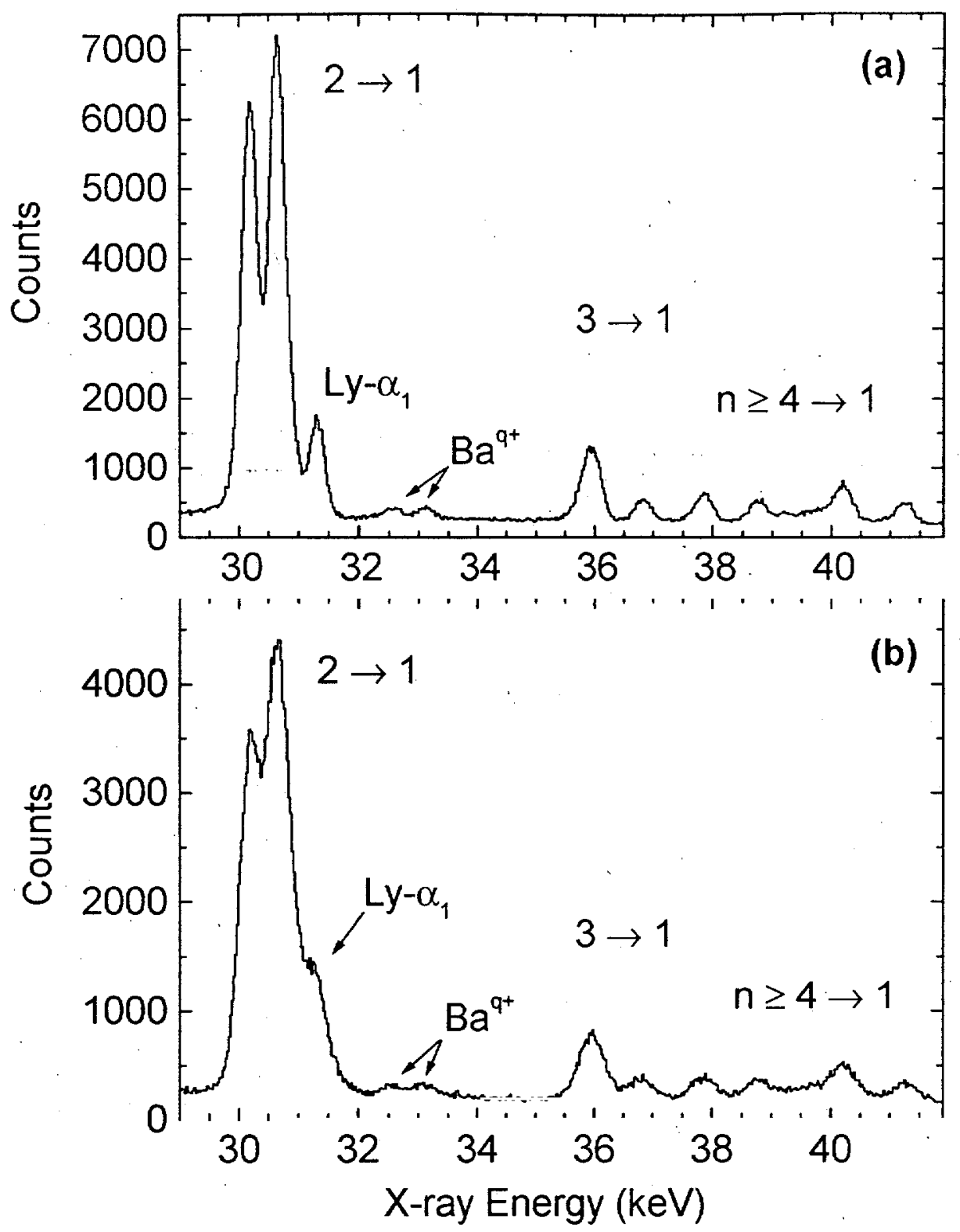

Figure 7.17: Rydberg series of the $\mathrm{n} \geq 2 \rightarrow 1$ transitions in highly charged xenon recorded with (a) a high-purity germanium detector (EGG) and (b) an intrinsic germanium detector (PGT). Both detectors have a planar germanium crystal geometry of equal size, i.e., a 16-mm diameter 10-mm deep cylinder. The measured resolution is about $270 \mathrm{eV}$ for the EGG and $360 \mathrm{eV}$ for the PGT detector. The spectral feature around $33 \mathrm{keV} \mathrm{x}$-ray energy are caused by highly charged barium that comes from the electron gun and, thus, is an impurity intrinsic to the SuperEBIT device. Each of the two spectra represents roughly three hours of data acquisition time. 


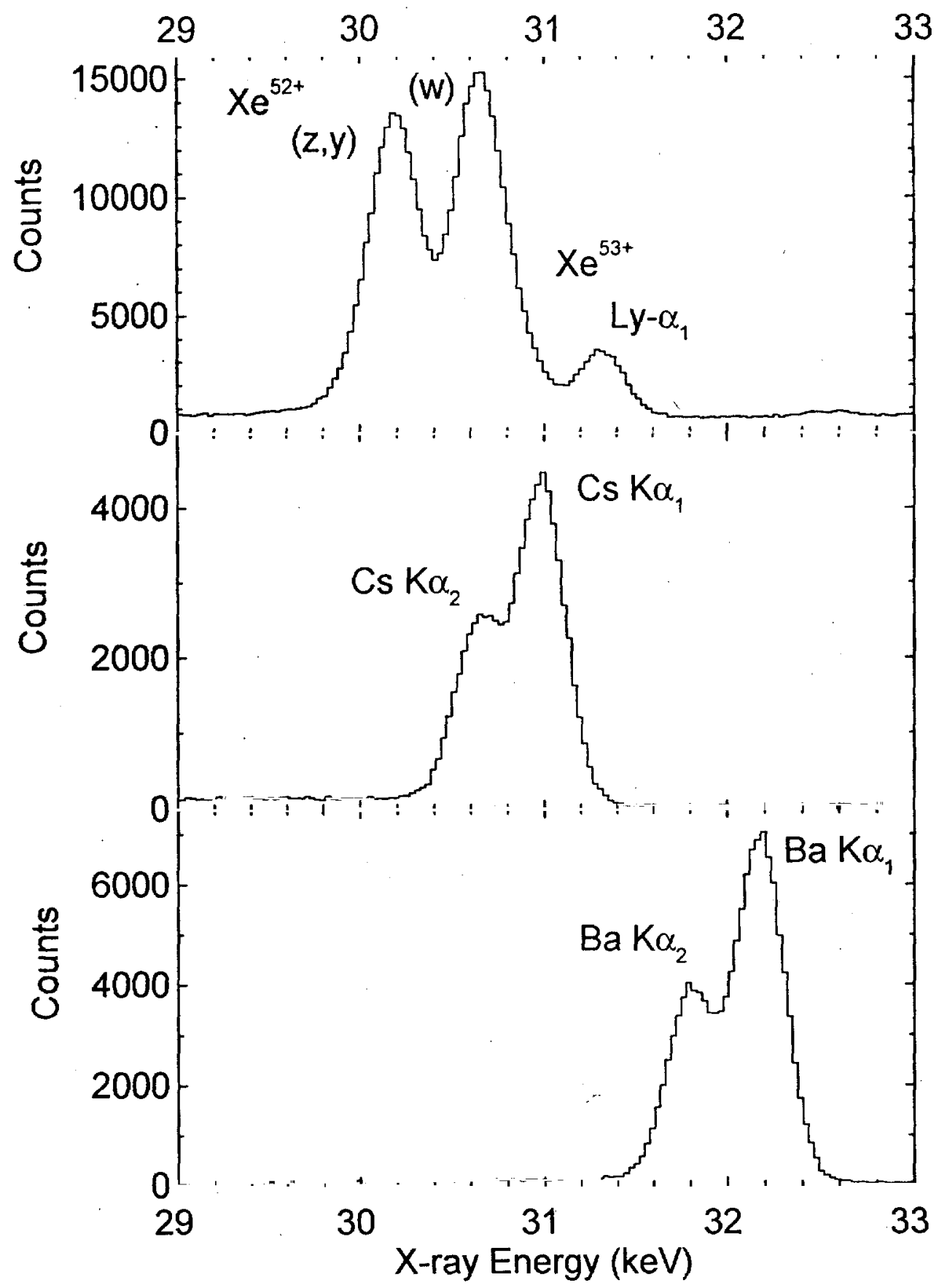

Figure 7.18: K $\alpha$ spectra of (a) highly charged xenon ions, (b) cesium, and (c) barium. The cesium fluorescence is emitted by a ${ }^{133} \mathrm{Ba}$ radioisotope, which after capturing a $\mathrm{K}$-shell electron converts into an innershell excited ${ }^{133} \mathrm{Cs}^{*}$. The barium $\mathrm{K} \alpha$ radiation is emitted by a barium foil which is illuminated with the radioisotope ${ }^{241} \mathrm{Am}$ causing $\mathrm{K}$-shell photoionization in the barium. All three spectra together represent about 12 hours of data acquisition time. 
the germanium detector, i.e, Compton profile and escape peak behavior, as discussed in Section 5.1 .2 on page 73 ). The line centroid values determined by fitting the EGG spectra with the germanium detector profile are plotted as a function of SuperEBIT runtime in Figure 7.19. The few times when the high voltage for the detector was turned off are indicated in this plot, too. The result shows that there are significant shifts over time in the positions of all lines. It clearly emphasizes the need of continuous calibration for high-precision measurements. Otherwise, this long-term drift of the pulse-height signal of the detector introduces a systematic uncertainty which is more than an order of magnitude bigger than the statistical uncertainty based on the number of counts in the spectral line.

In the present evaluation of the xenon Ly- $\alpha_{1}$ transition energy three different ways accounting for the line shifts are compared. One result is obtained assuming that the line shifts are purely statistical and, thus, using the weighted average of the measured line centroid positions for the evaluation of the dispersion and the transition energy. The other two results account for the significant line shifts using the measured time behavior of the line centroid positions for interpolation of the cesium $\mathrm{K} \alpha_{1,2}$ centroid positions with respect to the time marking the measurement of the barium and xenon spectra. A linear and a quadratic interpolation was applied for creating the time-shifted set of cesium line centroid positions. With the adjusted cesium and the barium line positions the dispersion of the detectors was determined. Both, a linear and a non-linear dispersion relation was evaluated. For the determination of the xenon Ly- $\alpha_{1}$ transition energy only the slope of the detector's energy dispersion is of importance. The offset, i.e., the zero-order coefficient, was inferred from adjacent cesium measurements. Additionally, all data taken with the EGG detector were also fitted with a Gaussian profile and the $L y-\alpha_{1}$ transition energy analyzed as described above. The impact of the different ways accounting for the line shape, the line shifts, and the detector dispersion on the result, i.e., the xenon $\mathrm{Ly}-\alpha_{1}$ transition energy values, can be seen in Table 7.20. 


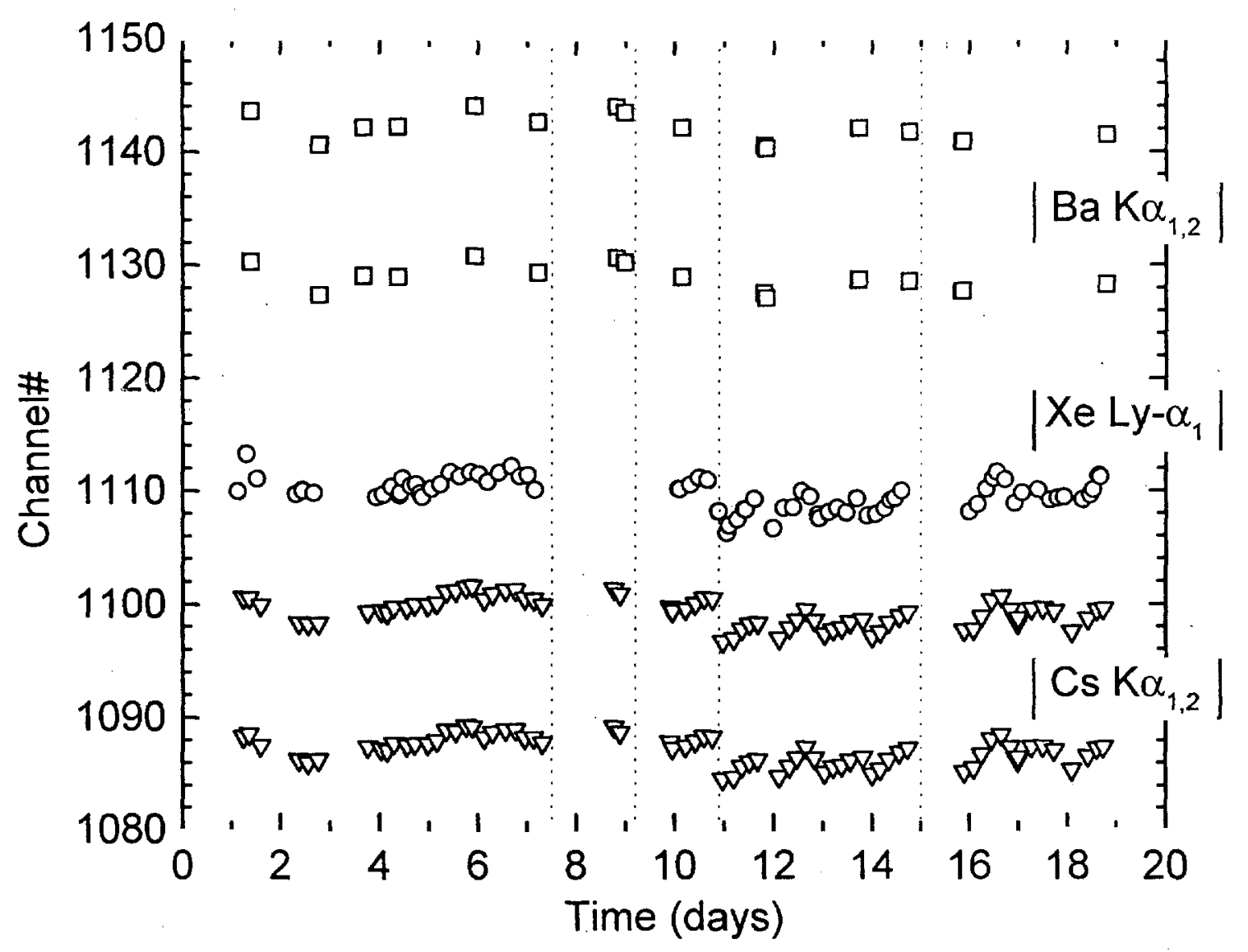

Figure 7.19: Centroid positions of the spectral lines given in the arbitrary quantity Channel Number recorded with the EGG germanium detector as a function of the SuperEBIT runtime, i.e., the duration of the experiment. The statistical uncertainties for the centroid positions are about the size of - in most cases actually smaller than - the symbols used in this plot. Thus, the shifts in the centroid positions are significant. The dashed lines indicate when the high voltage power supply for the EGG detector was turned off. The shutdown at the 11-day time mark was caused by a high-frequency transient in the electrical system.

The energy values for the calibration lines are from the CRC Handbook for Chemistry and Physics [143] and are listed in Table 7.19. These values are taken from a paper by Bearden published in $1967|144|$ and require correction due to changes in the value for the voltage-wavelength product $(V \lambda)$ and the value for the conversion factor of the wavelength units $\left.\left(\AA^{*} / \AA\right) \mid 145\right\}$. The $\AA^{*} / \AA$ correction incorporates also 
the most recent result of the $2 \mathrm{~d}$ spacing of the (220) oriented lattice planes in a nearly perfect silicon crystal $[146,147 \mid$ (see Table A.1, page 194). Additional adjustment is required for the uncertainties, which reflect the probable error (abbreviated as "p.e." in the wavelength and energy compilation by Bearden) which is defined as a $50 \%$ confidence limit and does not include the uncertainty of the $\AA^{*}$ unit due to the uncertainty of the $W K \alpha_{1}$ measurement. Even though the original paper |144| emphasizes the fact that Gaussian statistics was not applicable for most data, it seems to be the standard approach to convert the "p.e." values to a $67 \%$ confidence limit by assuming Gaussian distribution and, thus, to multiply the uncertainty values by a factor of 1.48 (e.g. $[148,149,118 \mid)$. For the calibration both the cesium and barium transition energy values were taken from the widely used compilation by Bearden. The most recent measurement available for the barium $\mathrm{K} \alpha$ transition energies $[150 \mid$ is also listed in Table 7.19. The implementation of this values for the barium $\mathrm{K} \alpha$ lines would cause a decrease in the measured hydrogenlike xenon line by less than $0.5 \mathrm{eV}$, i.e., less than $16 \mathrm{ppm}$.

The dispersion of the germanium detector was determined utilizing a linear and quadratic regression. The result of the non-linear regression gave a high uncertainty in the higher order terms showing that the constellation of the four calibration points $\mathrm{Cs} \mathrm{K} \alpha_{1,2}$ and $\mathrm{Ba} \mathrm{K} \alpha_{1,2}$ is sufficiently described by a linear regression. The high uncertainty in the non-linear terms propagates through the whole analysis and increases the uncertainty of the final result of the transition energy, as seen in Table 7.20. The first row of values in Table 7.20 was obtained by simply taking the weighted average of the measured line positions for the $\mathrm{Cs} \mathrm{K} \alpha_{1,2}$ lines, the $\mathrm{Ba} \mathrm{K} \alpha_{1,2}$ lines, and the $\mathrm{Xe}$ $\mathrm{Ly}-\alpha_{1}$ line. This approach does not account for the shifts of the centroid positions displayed in Figure 7.19. Neglecting this significant line shifts introduces a systematic uncertainty which is not included in the result of $(31273.1 \pm 5.3) \mathrm{eV}$. The big difference between the energy values obtained when applying a Gaussian profile instead of the germanium detector profile proves the significance of the impact on the symmetry 
Table 7.19: Transition energies of the cesium and barium calibration lines. The energy values in column $E_{C R C}$ are taken from the compilation published by Bearden [144]. These values are also listed in the Handbook of Chemistry and Physics [143]. $E_{a d j}$ are the adjusted energy values incorporating the current values for the x-ray standards [145] and the most recent measurement of the 2d spacing of the Si (220) crystal $[146,147]$. Only for the $\mathrm{K} \alpha$ emission of barium a more recently measured value is available, $E_{\text {rec }}[150]$. This value, too, was adjusted to the current x-ray standards.

\begin{tabular}{rccc}
\hline Line & $\begin{array}{c}E_{C R C} \\
\mathrm{eV}\end{array}$ & $\begin{array}{c}E_{a d j} \\
\mathrm{eV}\end{array}$ & $\begin{array}{c}E_{\text {rec }} \\
\mathrm{eV}\end{array}$ \\
\hline $\mathrm{Cs} \mathrm{K} \alpha_{2}$ & $30625.1(3)$ & $30626.32(44)$ & \\
$\mathrm{Cs} \mathrm{K} \alpha_{1}$ & $30972.8(3)$ & $30974.06(46)$ & \\
$\mathrm{Ba} \mathrm{K} \alpha_{2}$ & $31817.1(4)$ & $31818.39(61)$ & $31816.62(6)$ \\
$\mathrm{Ba} \mathrm{K} \alpha_{1}$ & $32193.6(3)$ & $32194.89(50)$ & $32193.27(7)$ \\
\hline
\end{tabular}

of the measured line profile due to the detection mechanism of a germanium detector. In fact, the lower resolution of the PGT detector did not allow a successful fit of the recorded spectra when using a Gaussian profile. But even when applying the germanium detector profile the results obtained with the PGT detector are unsatisfactory due to the rather poor resolution of the spectra. Therefore, no result was obtained using a non-linear line position interpolation and detector dispersion determination for the PGT data.

Accounting for the shift of the centroid positions improves the result with respect to the uncertainty indicating less scatter of the individual transition energy values obtained for each xenon spectrum. The difference between linear and non-linear interpolation of the line centroid position is within the uncertainty of the measured transition energy values. This indicates that additional refinement of the function 
Table 7.20: Comparison of the results for the xenon $\mathrm{Ly}-\alpha_{1}$ transition energy. The different results are obtained by fitting the recorded lines with a Gaussian profile (Gauß) and a germanium detector profile (Ge-det). Neglecting the shifts of the line centroids over time yields the "none" values. Applying a linear and a non-linear interpolation for the determination of the time dependence of the line centroid positions reduces the scattering of each value and, thus, reduces the uncertainty of the results. The "none" and "linear" values are based on a linear dispersion of the germanium detector. The "non-linear" results also implement a non-linear dispersion. The increased uncertainty arises from the uncertainties of the higher order terms in the non-linear dispersion showing that the constellation of the four calibration points $\mathrm{Cs} \mathrm{K} \alpha_{1,2}$ and $\mathrm{Ba} \mathrm{K} \alpha_{1,2}$ is sufficiently described by a linear regression. The large deviation of the PGT result from the theoretical value is not surprising considering the rather poor resolution (see Figure 7.17). The implementation of the more recent energy values for the barium $\mathrm{K} \alpha$ lines (listed in Table 7.19) would cause a decrease in the measured hydrogenlike xenon line by less than $0.5 \mathrm{eV}$, i.e., less than 16 ppm.

\begin{tabular}{rccc}
\hline \hline \multicolumn{4}{c}{$\mathrm{Xe}^{53+} \mathrm{Ly}-\alpha_{1}$ transition energy $(\mathrm{eV})$} \\
\hline \multicolumn{3}{c}{ EGG } & PGT \\
& Gauß & Ge-det & Ge-det \\
\hline none & $31266.9 \pm 4.2$ & $31273.1 \pm 5.3$ & $31267.9 \pm 5.5$ \\
linear & $31271.3 \pm 4.0$ & $31279.2 \pm 1.5$ & $31266.0 \pm 4.1$ \\
non-linear & $31270.6 \pm 5.3$ & $31278.5 \pm 1.7$ & - \\
\hline Theoretical prediction: & $31283.77 \pm 0.09$ \\
\hline \hline
\end{tabular}

describing the time dependence of the line positions is not necessary. 


\subsubsection{Discussion}

The following discussion focuses on the result with the smallest uncertainty, i.e., the measured xenon Ly- $\alpha_{1}$ transition energy of $(31279.2 \pm 1.5) \mathrm{eV}$ obtained applying a germanium detector line profile, linear dispersion, and linear interpolation for adjusting the centroid positions of the calibration lines with respect to the time of measurement. Although this value is closest to the theoretical prediction of Johnson and Soff $[27]$, i.e., $E_{\text {theo }}=(31283.77 \pm 0.09)$, there is still a significant disagreement of $4.6 \mathrm{eV}(\approx 150 \mathrm{ppm})$ between the measured and the theoretical value, which is more than three times the 1- $\sigma$ confidence limit of the measurement. Such a result is very surprising since the atomic structure of hydrogenlike system is well understood. That well, in fact, that it is common practice using theoretical predictions for the transition energies as a calibration reference $[151,152]$.

Since the measured value depends on the transition energy values used for the calibration lines, the disagreement could very well arise from those cesium and barium $\mathrm{K} \alpha$ transition energy values listed in Table 7.19. As mentioned above, those values are from an $\mathrm{x}$-ray wavelengths compilation published by Bearden |144|. In this paper Bearden discusses the difficulty of the line centroid assignment due to some asymmetry of the measured line profiles. Taking the position of the line peak instead of the line centroid seemed at that time the best solution. Thus, strictly speaking those values are only applicable if the experimental setup, i.e., the spectrometer and the $\mathrm{x}$ ray source, reproduces exactly the same line profile and radiation, that was observed in the measurement which the calibration values are based on and, thus, the line peaks can be evaluated instead of the line centroids. Even under the assumption that those calibration spectra contained lines with a symmetric profile and, therefore, the peak and centroid position are identical, the compatibility is not guaranteed unless the same type of $\mathrm{x}$-ray source is used. The standard equipment for those calibration measurements was a flat-crystal spectrometers and an $\mathrm{x}$-ray tube. The calibration in the present measurement, however, is based on the characteristic $\mathrm{K} \alpha \mathrm{x}$-ray fluo- 
rescence involving radioisotopes. In particular, the cesium $\mathrm{K} \alpha$ radiation is emitted by the radioisotope ${ }^{133} \mathrm{Ba}$ after a $\mathrm{K}$-shell electron capture reaction, and the barium $\mathrm{K} \alpha$ fluorescence is the radiative decay after photoionization due to illumination of the barium foil with the radioisotope ${ }^{241} \mathrm{Am}$. In X-ray tubes, however, the characteristic $\mathrm{K} \alpha \mathrm{x}$-ray fluorescence is emitted in response to electron-impact ionization and perhaps electron-impact excitation. The energy density deposited into the target by electrons is much higher than by photons and, thus, shake-up and shake-off processes are more common in an $\mathrm{x}$-ray tube target than in a radioisotope based $\mathrm{x}$-ray source $[153,154 \mid$. These changes in the excitation and ionization stages, naturally, shift the energy of the emitted characteristic $x$ rays. Moreover, the heat load onto the anode in an $\mathrm{x}$-ray tube requires sometimes the use of a more heat resistive compound, which causes a shift in the energy of the emitted characteristic $x$-rays, too. The change in the level structure due to the surrounding material might be an important argument against the use of the ${ }^{133} \mathrm{Ba}$ radioisotope for high precision measurements utilizing the characteristic cesium $\mathrm{K} \alpha$ fluorescence.

Additionally, the position of the line centroid can be shifted due to scattered photons which have a slightly lower energy. The design of the calibration stand used for this measurement, for example, did not include any aperture in order to reduce the intensity of characteristic $\mathrm{x}$ rays which reach the detector after being scattered from the wall. The perfect design, of course, would be a replication of the SuperEBIT setup with respect to the geometry and the materials. Such a calibration stand, thus, would provide the same conditions for emitted cesium and barium $\mathrm{x}$ rays, as there are for the $\mathrm{x}$ rays emitted by the highly charged xenon ions.

The decision whether the disagreement between the measured and the theoretical value for the xenon $\mathrm{Ly}-\alpha_{1}$ transition energy is due to inaccurate transition energy values of the calibration lines, or a reevaluation of the theory for the atomic structure of the hydrogenlike ions is necessary, depends on the availability of high-precision measurements regarding the characteristic cesium $\mathrm{K} \alpha \mathrm{x}$ rays emitted by the ${ }^{133} \mathrm{Ba}$ ra- 
dioisotope. This measurement, however, certainly proves that continuous calibration of the measured spectra is necessary to account for long-time drifts of the detector response. These significant shifts of the line centroid position over time also limit the maximum accuracy achievable with those germanium detectors. Estimating the accuracy using plain statistical considerations gives an uncertainty of only $\pm 0.16 \mathrm{eV}$ for the Ly- $\alpha_{1}$ transition energy measured with the EGG detector which accumulated a total of 526000 counts in the hydrogenlike xenon line. This shows that the uncertainty is not at all limited by counting statistics and further improvement with respect to the accuracy requires the implementation of instruments with a higher resolving power.

\subsection{Conclusion}

The current measurement opened a window on high-resolution hard $\mathrm{x}$-ray spectroscopy of highly charged ions. All previous measurements involving the $\mathrm{n}=2 \rightarrow 1$ transitions in highly charged xenon were based on solid state detectors $\{155,156,134\}$ - The low resolution of these detectors in comparison with a crystal spectrometer limits the achievable accuracy as demonstrated in Section 7.2. Although the current measurement of the xenon $L y-\alpha_{1}$ transition energy is about six times more accurate than the so far most precise measured value [155], it actually shows that further refinement in the experimentally achieved transition energy values requires spectrometers with a much higher resolving power than it is offered by solid state detectors.

The successful implementation of such a high-resolution spectrometer is demonstrated in Section 7.1. The instrument, a transmission-type crystal spectrometer, shows a ten times higher resolving power than the best germanium detector currently available. The high resolution enables the separation of the heliumlike transitions $\mathrm{z}$ $\left(1 s 2 s^{3} S_{1} \rightarrow 1 s^{2}{ }^{1} S_{0}\right)$ and $\mathrm{y}\left(1 s 2 p^{3} P_{1} \rightarrow 1 s^{2}{ }^{1} S_{0}\right)$, and of the heliumlike transition $\mathrm{w}$ $\left(1 s 2 p^{1} P_{1} \rightarrow 1 s^{2} S_{0}\right)$, the lithiumlike transition $\mathrm{q}\left(1 s 2 s 2 p^{2} P_{3 / 2} \rightarrow 1 s^{2} 2 s^{2} S_{1 / 2}\right)$ and the hydrogenlike Ly- $\alpha_{2,3}$ blend $\left(2 p_{1 / 2} \rightarrow 1 s_{1 / 2}, 2 s_{1 / 2} \rightarrow 1 s_{1 / 2}\right.$. Even though the mea- 
sured cross sections have uncertainties above $10 \%$, significant disagreement is found between the measured and theoretically predicted values for the transitions in heliumlike $\mathrm{Xe}^{52+}$. The theoretical values are fully relativistic distorted wave function calculations, which do not include the Breit interaction. The measurement shows, however, that the contribution due to the Breit interaction between the two bound and the free electron must be of significant magnitude. Preliminary results ${ }^{38}$ of calculations including the Breit interaction according to $[5]$ are in perfect agreement with the measured values.

The crystal curvature is currently the limiting factor with respect to the resolving power. Instrumental improvements must focus on a different way of bending the transmission crystal, such as using a preshaped foam mask which hosts the crystal in a sandwich-type arrangement.

\footnotetext{
${ }^{38}$ Honglin Zhang, private communications.
} 


\section{Summary}

\subsection{Results}

This work successfully demonstrated the feasibility of extending spectroscopical plasma diagnostic tools to the hard $x$-ray regime. The results of the high-resolution spectroscopic measurements on highly charged krypton and xenon include the first excitationenergy selective measurement of the dielectronic recombination resonances in the heliumlike krypton $\mathrm{K} \alpha$ spectrum, the first level-specific measurement of the $\mathrm{n}=2 \rightarrow 1$ transitions in heliumlike and hydrogenlike xenon, and the first determination of the electron-impact excitation cross section in heliumlike systems that are heavier than iron, i.e., with an atomic number $\mathrm{Z}>26$. In particular:

Krypton: The excitation-energy selective spectra, presented in Figure 6.3, page 119 , enable the separation of transitions that have the same $\mathrm{x}$-ray energy but strongly depend on the excitation energy.

- The measurement of the wavelengths and transition energies supports the latest development of the atomic structure calculations of heliumlike systems and shows the need for a thorough treatment of the QED effects. The presented transition-energy measurements have an accuracy of about $30 \mathrm{ppm}$ and, therefore, are sensitive to test the 1.4-eV QED contribution to the energy of the $1 s 2 s$ configuration [152]. The wavelengths calibration of the krypton spectra was performed using well known lines of the $\mathrm{K} \alpha$ emission of hydrogenlike manganese and heliumlike and berylliumlike iron.

- The excitation-energy selective spectra show that the "forbidden" transition $1 s 2 s^{3} S_{1} \rightarrow 1 s^{2}{ }^{1} S_{0}$, i.e., line $\mathrm{z}$ in Figure 6.3, is the best suited candidate among the heliumlike transitions for the determination of the ion temperature. This preference arises from the relatively small overlap - regarding 
the $x$-ray energy - with dielectronic satellite lines.

- The KLL (1s2s2l excited level) DR resonances in lithiumlike krypton were identified and their wavelengths measured (see Table 6:3, page 122). The wavelengths are determined relative to the heliumlike transitions.

Xenon: The development of a DuMond-type transmission crystal spectrometer was the base of the experimental effort presented in Chapter 7. The low $x$-ray flux emitted by SuperEBIT requires special care with respect to the instrumental efficiency. Utilizing a transmission-type crystal and operating the spectrometer in monochromator mode, thus, providing almost $100 \%$ detection efficiency by employing a germanium detector, were important steps in achieving the spectra of heliumlike and hydrogenlike xenon shown in the Figures 7.3 and 7.4. The investigation of the xenon spectra includes the line positions for the determination of the transition energies, and the line intensities which were used for the determination of the electron-impact cross sections.

- The $K \alpha$ transition energies were determined relative to the transition energy of the xenon $\mathrm{Ly}-\alpha_{1}$ line. The precision is in the 3 to $6-\mathrm{eV}$ range and is limited by counting statistics.

- Utilizing two germanium detectors, the xenon Ly- $\alpha_{1}$ transition energy was measured with respect to the current $\mathrm{x}$-ray standard. This measurement, motivated by the need for a calibration of the recorded transmission spectra, shows the impact of the detector's long-time stability on the achievable precision, in particular, $50 \mathrm{ppm}$ whereas the uncertainty due to counting statistics was only $5 \mathrm{ppm}$. The measurement demonstrates the limitation of low-resolution devices and, thus, emphasizes the need of high-resolution spectrometers. The disagreement between the measured xenon $L y-\alpha_{1}$ transition energy and the theoretical prediction is somewhat surprising. However, this disagreement does not necessarily call for a correction in respect 
to the widely accepted theoretical values for the transition energies of the hydrogenlike isoelectronic sequence. The difference most likely reflects the shift between the line centroid and the peak position due to the asymmetry of the line profile in the spectrum the cesium calibration values are extracted from. Therefore, a reevaluation of the commonly used x-ray wavelengths table for atomic inner-shell transitions is required.

- The determination of the electron-impact cross sections relative to the radiative recombination cross sections is a very successful demonstration of the functionality of the developed transmission-type spectrometer. Additionally, these measured cross sections benchmark the development of atomic codes in the medium and high- $\mathrm{Z}$ regime. In particular, the measured values show the significance of the Breit interaction in this electronenergy regime and, therefore, the importance of including the Breit interaction in the calculations.

EBIT/SuperEBIT: The discussion of the electron beam properties, which includes the relativistic treatment of the electron motion ${ }^{39}$, lead to the derivation of the space charge assuming a Gaussian electron density distribution, see eq. (4.25) on page 35. By applying a simple model it was shown (Figure 4.8, page 41 ), that due to the drift tube geometry the electron beam always creates an axial trapping potential even without applying a potential difference between the drift tubes. Additionally, the space charge compensation factor was inferred from two measurements, presented in Table 4.4, and on page 149.

\footnotetext{
${ }^{39}$ None of the published papers that include a discussion about the electron-beam properties, such as electron density or space charge potential, of EBIT or SuperEBIT apply relativity.
} 


\subsection{Suggestions for future research}

There is a wealth of information contained in the krypton spectra, which has not been exploited so far. The goal is the identification of all dielectronic resonances, and the determination of their position but also of their relative intensities. Such a complete set of experimentally achieved atomic data is imperative for correct and accurate interpretation of spectra obtained from the various plasma sources as well as for correct predictions of the energy transfer in plasmas by means of radiation and electron-ion collisions.

The charge exchange cross sections between highly charged xenon ions and neutral neon atoms, presented in Table 7.13, show a very interesting pattern, namely, that the charge exchange between the neutral neon atoms and hydrogenlike xenon ions is definitely favored compared to the charge exchange between the neutrals and bare or heliumlike ions. A verification of this result is desirable and might shed some light on the dependence of the charge exchange process on the total angular momentum of the highly charged target ions.

The measurement of the xenon Ly- $\alpha_{1}$ transition energy using the germanium detectors indicates that the tabulated transition energy values of the characteristic $\mathrm{K} \alpha \mathrm{x}$. rays of some elements have a systematic uncertainty due to the definition of the transition energy and wavelength, respectively, as the peak value of the spectral feature. This definition does not account for the asymmetric appearance in the measurement the tabulated values are based on. High-precision measurements, thus, might have to be based on a different calibration, as presented in the measurement of the transition energies in heliumlike krypton, i.e., the calibration with respect to hydrogenlike Ly- $\alpha$ $\mathrm{x}$ rays. In fact, the theoretically predicted values might reach such a precision that hydrogenlike lines could be used as the new $\mathrm{x}$-ray standards. 


\subsubsection{Instrumental improvements}

- SuperEBIT: Replacing the kerosene cooling system with a less corrosive liquid, e.g., purified water, which is the prime candidate with respect to the environment, would certainly improve the reliability of the SuperEBIT device operation.

- Position sensitive proportional counter: Implementation of a resistive delay line on each side of the anode wire array would add a second spatially resolved dimension. A two-dimensional detection of the spectrally decomposed image of the electron beam is important for a von Hámos spectrometer setup when crystals with a small radius of curvature are used, as shown in Figure 5.6 on page 85 .

- Crystal bender: The variable crystal bender, designed for the transmission-type spectrometer, offers too many degrees of freedom for crystals that have a small elasticity module, i.e., crystals that easily deform plastically, such as lithium fluoride. A new crystal bender was designed, where the crystal is basically pressed into the desired shape by a set of prefabricated blocks made out of a low-Z, low density material. The new design, however, has not been tested, yet.

- Transmission-type spectrometer: An advanced design of the developed crystal spectrometer could host a set of crystals behind each other, since the nonresonant transmission of hard $\mathrm{x}$-rays is very high due to the thinness of the crystal. Thus, different crystals could "filter" different $x$-ray energies. 


\section{A Table of physical constants}

Table A.1: List of fundamental physical constants used in this thesis. Unless specified the values are taken from Cohen and Taylor [145]. More recent values were incorporated if they had a higher or equal precision. The column symbol gives, of course, only the most common notation used for the specific quantities. The column $\Delta_{\text {rel }}$ lists the relative uncertainties.

Quantity Symbol Values \& Units $\quad \Delta_{\text {rel }} \quad$ Ref.

\begin{tabular}{|c|c|c|c|c|}
\hline & & & ppm & \\
\hline speed of light in vacuum & $c_{0}$ & $299792458 \frac{\mathrm{m}}{\mathrm{s}}$ & (exact) & \\
\hline permeability of vacuum & $\mu_{0}$ & $4 \pi \cdot 10^{-7} \frac{\mathrm{Vs}}{\mathrm{Am}}$ & (exact) & \\
\hline permittivity of vacuum & $\epsilon_{0}$ & $8.854187817 \ldots \cdot 10^{-12} \frac{\mathrm{As}}{\mathrm{Vm}}$ & (exact) & \\
\hline Planck constant & $h$ & $6.6260755 \cdot 10^{-34} \mathrm{Js}$ & 0.60 & \\
\hline elementary charge & $e$ & $1.60217733 \cdot 10^{-19}$ As & 0.30 & \\
\hline electron mass & $c_{0}$ & $9.1093897 \cdot 10^{-31} \mathrm{~kg}$ & 0.59 & \\
\hline Boltzmann constant & $k$ & $1.380658 \cdot 10^{-23} \frac{J}{K}$ & 8.5 & \\
\hline Rydberg constant & $R_{\infty}$ & $1.0973731534 \cdot 10^{8} \frac{1}{m}$ & 0.0012 & \\
\hline wavelength voltage product & $\lambda V$ & $12398.4243 \mathrm{eVA}$ & 0.60 & \\
\hline$X$-ray wavelength unit & $\AA^{*}$ & $\lambda\left(\mathrm{W} \mathrm{K} \alpha_{1}\right) \equiv 0.2091 \AA^{*}$ & & $\lceil 144\rceil$ \\
\hline$\AA^{*}$ unit & $\AA^{*}$ & $1.00001481 \cdot 10^{-10} \mathrm{~m}$ & 0.92 & \\
\hline lattice spacing of $\operatorname{Si}(220)$ in & $d_{220}$ & $1.92015540 \cdot 10^{-10} \mathrm{~m}$ & 0.21 & {$[146,147]$} \\
\hline
\end{tabular}




\section{B Velocity of electrons accelerated by an electro- static field}

The kinetic energy of an electron is equal to the electron charge times the acceleration potential, i.e., $E_{k i n}=E_{\text {pot }}=e U$, where $e$ is the elementary charge and $U$ the acceleration potential. Generally, the kinetic energy can be expressed as the gain in the electron mass $(\Delta m)$ times the speed of light in vacuum square $\left(c_{0}{ }^{2}\right)$, i.e.,

$$
\begin{aligned}
E_{k i n} & =\Delta m c_{0}^{2}=m_{0} c_{0}^{2}(\gamma-1) \\
\text { with } \gamma & =\frac{1}{\sqrt{1-\left(\frac{v}{c_{0}}\right)^{2}}} .
\end{aligned}
$$

Thus, the speed of the electron is:

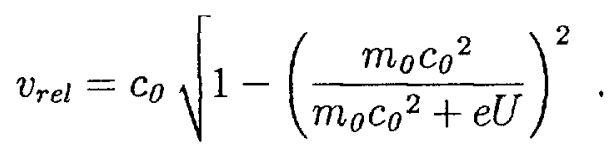

In the non-relativistic case where the electron speed is much less than the speed of light the factor $\gamma$ can be sufficiently characterized by following expansion,

$$
\gamma=\left[1-\left(\frac{v}{c_{0}}\right)^{2}\right]^{-\frac{1}{2}} \approx 1+\frac{v^{2}}{2 c_{0}^{2}}
$$

and eq.(B.3) modifies to:

$$
v_{c l}=\sqrt{\frac{2 e U}{m_{0}}} .
$$

In Figure B.1 the electron speeds obtained with the relativistic and non-relativistic formulae are compared, and it clearly shows the systematic overestimation of the electron speed when using the non-relativistic relation. Moreover, it shows that above an acceleration potential of $U=\frac{m_{0} c_{0}^{2}}{2 e} \approx \frac{511}{2} \mathrm{kV}$ the classic treatment yields electron velocities that exceed the speed of light. For practical purposes, it is very handy to express the acceleration voltage in terms of the relative systematic uncertainty introduced when using the non-relativistic approach. Defining the relative systematic 
uncertainty as

$$
\Delta_{v}=\frac{v_{c l}-v_{r e l}}{v_{r e l}}
$$

the upper limit of the acceleration voltage in order to not exceed a given relative systematic uncertainty is:

$$
U_{\max }=\frac{m_{o} \dot{c}^{2}}{e}\left\{-1+\frac{\left(1+\Delta_{v}\right)^{2}}{4}+\sqrt{\left(1+\Delta_{v}\right)^{2}\left[\frac{1}{2}+\frac{\left(1+\Delta_{v}\right)^{2}}{16}\right]}\right. \text {. }
$$

If, for example, the relative systematic uncertainty of the calculated electron velocity should be below 1\%, then the classic approach is limited to acceleration potentials below $6.8 \mathrm{kV}$. A graph showing the dependence of the maximum acceleration voltage on the limit of relative systematic uncertainty is shown in Figure B.1.

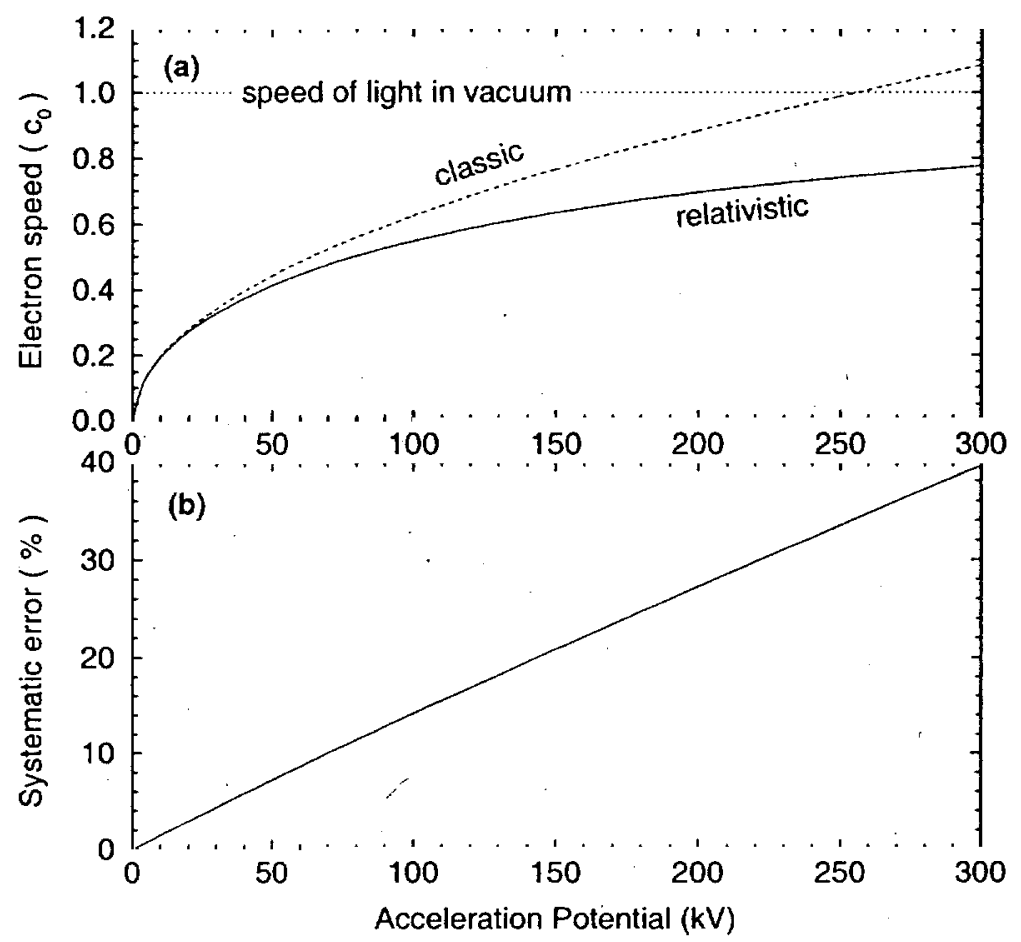

Figure B.1: Comparison of relativistic versus non-relativistic treatment for determination of the electron speed in the presence of a acceleration potential $U$. Graph (a) shows the overestimation of the electron velocity (given as fraction of the speed of light in vacuum) when applying classical mechanics. (b) gives the difference between relativistic and non-relativistic calculation in percent (refer to eq.(B.6)). 


\section{References}

11 Ludwig Bergmann and Clemens Schaefer. Lehrbuch der Experimentalphysik: Band V, Vielteilchen-Systeme, chapter 2 "Plasmen", by Joachim Seidel and Burkhard Wende, pages 97-225. Walter de Gruyter, Berlin, 1992. Herausgeber: Wilhelm Rath.

[2] Ian H. Hutchinson. Principles of Plasma Diagnostics. Cambridge University Press, 1987. ISBN 0-521-38583-0.

[3| E. Källne, J. Källne, E.S. Marmar, and J.E. Rice. High-resolution x-ray spectroscopy diagnostics of high temperature plasmas. Phys. Scr., 31:551-564, 1985.

$14 \mid$ P.T. Springer, K.L. Wong, C.A. Iglesias, J.H. Hammer, J.L. Porter, A. Toor, W.H. Goldstein, B.G. Wilson, F.J. Rogers, C. Deeney, D.S. Dearborn, C. Bruns, J. Emig, and R.E. Stewart. Laboratory measurement of opacity for stellar envelopes. J. Quant. Spectrosc. Radiat. Transfer, 58(4-6):927-935, Oct.-Dec. 1997.

[5] Christopher J. Fontes, Douglas H. Sampson, and Hong Lin Zhang. Inclusion of the generalized breit interaction in excitation of highly charged ions by electron impact. Phys. Rev. A, 47(2):1009-1022, February 1993.

[6] B. Edlén and A. Ericson. Nature, 125:233, 1930.

17] J.H. Parkinson. The analysis of a high resolution $\mathrm{x}$-ray spectrum of a solar active region. Solar Physics, 42:183-207, 1975.

[8] D.L. McKenzie. P.B. Landecker, U. Feldman, and G.A. Doschek. The solar coronal x-ray spectrum from 5.5 to $12 \AA$. Astrophys. J., 289:849-857, February 1985.

[9] I.A. Zhitnik, V.V. Korneev, V.V. Krutov, S.N. Oparin, and A.M. Urnov. Highresolution spectroscopy of the solar $\mathrm{x}$-ray spectrum. In I.I. Sobel'man, editor, 
X-ray Plasma Spectroscopy and the Porperties of Multiply Charged Ions, pages 51-77, Commack, New York, 1988. Academy of Science of the USSR, NOVA Science Publishers Inc. Proceedings of the Lebedev Physics Institute, translated by Kevin S. Hendzel.

[10] Peter Beiersdorfer. High-resolution studies of the $x$-ray transitions in highly charged neonlike ions on the PLT tokamak. Dissertation, Princeton University, February 1988.

[11] Y.L. Li, G. Pretzler, and E.E. Fill. Ne-like ion lasers in the extreme ultraviolet region. Phys. Rev. A, 52(5):R3433-R3435, November 1995. Rapid Communications, Note.

[12] Joseph Nilsen, Peter Beiersdorfer, Klaus Widmann, Vincent Decaux, and Steven R. Elliott. Energies of neon-like $n=4$ to $n=2$ resonance lines. Phys. Scr., 54:183-187, 1996.

|13| Keith M. Matzen. Z pinches as intense $\mathrm{x}$-ray sources for high-energy density physics applications. Physics of Plasmas, 4(5):1519-1527, May 1997. Proceedings of the 38th annual meeting of the Division of Plasma Physic of the American Physical Society, Denver, Nov. 1996.

[14] A'. Fowler. Series lines in spark spectra. Roy. Soc. Proc., 90:426, 1914.

[15] F. Paschen. Bohrs Heliumlinien. Ann. d. Phys. (Leipzig), 50:901, 1916.

|16 M. Siegbahn and W. Stenström. Über die Hochfrequenzspektra (K-Reihe) der Elemente Cr bis Ge. Physikal. Zeitschr., 17:48, 1916.

[17] F. Paschen. Die Funkenspektren des Aluminium I. Ann. d. Phys. (Leipzig). $71: 142,1923$.

[18] A. Fowler. The spectra of the lighter elements. Nature, 113:219-223, 1924. 
|19| A. Sommerfeld and G. Wentzel. Über reguläre and irreguläre Dubletts. Z. Phys., 7:86-92, 1921.

|20| G. Wentzel. Funkenspektren im Röntgenspektrum. Ann. d. Phys. (Leipzig), $66: 437-462,1921$.

[21] G. Wentzel. Funkenspektren im Röntgenspektrum (Nachträge). Ann. d. Phys. (Leipzig), 73:647-650, 1924.

|22| A. Smekal. Die Feinstruktur der Röntgenspektren. Verhandl. d. dtsch. physik. Ges., 1:58, 1920. Proceedings of the German Physical Society.

[23] A. Smekal. Zur Feinstruktur der Röntgenspektren. Z. Phys., 5:91,121, 1921.

[24] A. Sommerfeld. Bemerkungen zur Feinstruktur der Röntgenspektren. Z. Phys., $1: 135,1920$.

[25] A. Sommerfeld. Bemerkungen zur Feinstruktur der Röntgenspektren 2. Z. Phys., 5:1, 1921.

[26] Arnold Sommerfeld. Atombau und Spektrallinien, volume I. Verlag Harri Deutsch, Frankfurt/M., Germany, 8 edition, 1978. ISBN 3-87144-484-7.

[27] W.R. Johnson and G. Soff. The Lamb shift in hydrogen-like atoms, $1 \leq Z \leq$ 100. At. Data Nucl. Data Tables, 33(3):405-446, November 1985.

[28] Willis E. Lamb Jr. and R.C. Retherford. Fine structure of the hydrogen atom by a microwave method. Phys. Rev., 72(3):241-243, August 1947.

[29| Jonathan R. Sapirstein and Donald R. Yennie. Theory of hydrogenic bound states. In T. Kinoshita, editor, Quantum Electrodynamics, volume 7 of Advanced Series on Directions in High Energy Physics, pages 560-672, Singapore, 1990. World Scientific, Utopia Press. ISBN 981-02-0213-X. 
[30| Heinrich F. Beyer, H.-Jürgen Kluge, and Viatcheslav P. Shevelko. X-Ray Radiation of Highly Charged Ions, chapter 3 "Atomic Structure and Spectra", pages 61-84. Atoms and Plasmas. Springer, Heidelberg, 1997. Comparison of Lamb-shift measurements in Table 3.16, pg. 82.

[31] H. Persson, S. Salomonson, P. Sunnergren, I. Lindgren, and M.G.H. Gustavsson. A theoretical survey of QED tests in highly charged ions. Hyperfine Inter., 108:3-17, 1997. 2nd Euroconference on Atomic Physics with Stored Highly Charged Ions, Stockholm June 1996.

[32| S.R. Elliott, P. Beiersdorfer, and M.H. Chen. Trapped-ion technique for measuring the nuclear charge radii of highly charged radioactive isotopes. Phys. Rev. Lett., 76:1031-1034, 1996.

[33] J.R. Crespo López-Urrutia, P. Beiersdorfer, D.W. Savin, and K. Widmann. Direct observation of the spontaneous emission of the hyperfine transition $\mathrm{F}=4$ to $\mathrm{F}=3$ in ground state hydrogenlike ${ }^{165} \mathrm{Ho}^{66+}$ in an electron beam ion trap. Phys. Rev. Lett., 77(5):826-829, July 1996.

[34] P. Beiersdorfer, S.R. Elliott, J.R. Crespo López-Urrutia, and K. Widmann. Measurements of nuclear parameters of high- $Z$ isotopes performed on a highenergy electron beam ion trap. Nucl. Phys. A, 626(1-2):C357-C364, November 1997.

[35] J.R. Crespo López-Urrutia, P. Beiersdorfer, K. Widmann, B.B. Birkett, A.-M. Mårtensson-Pendrill, and M.G.H. Gustavsson. Nuclear magnetization distribution radii determinsed by hyperfine transitions in the 1s level of H-like ions ${ }^{185} \mathrm{Re}^{74+}$ and ${ }^{187} \mathrm{Re}^{74+}$. Phys. Rev. A, 57(2):879-887, February 1998.

[36 G.W. Drake. Theoretical energies for the $n=1$ and 2 states of the helium isoelectronic sequence up to $\mathrm{Z}=100$. Can. J. Phys., 66:586-611, 1988. 
[37] D.R. Plante, W.R. Johnson, and J. Sapirstein. Relativistic all-order many-body calculations of the $\mathrm{n}=1$ and $\mathrm{n}=2$ states of heliumlike ions. Phys. Rev. A, 49(5):3519-3530, May 1994.

|38| K.T. Cheng, W.R. Johnson, and J. Sapirstein. Screened Lamb shift calculations for lithiumlike uranium, sodiumlike platinum, and copperlike gold. Phys. Rev. Lett., 66(23):2960-2963, June 1991.

[39] K.T. Cheng, W.R. Johnson, and J. Sapirstein. Lamb-shift calculations for non-coulomb potentials. Phys. Rev. A, 47(3):1817-1823, March 1993.

[40] Alan Burgess. Dielectronic recombination and the temperature of the solar corona. Astrophys. J., 139(2):776-780, 1964.

[41] A.H. Gabriel. Dielectronic satellite spectra for highly-charged helium-like ion lines. Mon. Not. R. astr. Soc., 160:99-119, 1972.

[42] P. Sortais. General review of recent developments for electron cyclotron resonance ion sources. Rev. Sci. Instrum., 67(3):867-872, March 1996.

|43| Heinrich F. Beyer, H.-Jürgen Kluge, and Viatcheslav P. Shevelko. X-Ray Radiation of Highly Charged Ions. Atoms and Plasmas. Springer, Heidelberg, 1997. ISBN 3-540-63185-2.

|44| L. Liljeby, editor. Proceedings of the 6th International Symposium on Electron Beam Ion Sources and their Applications, Physica Scripta T71. European Physical Society, 1997. Stockholm, Sweden, June 20-23, 1994.

45] Marylin B. Schneider, Morton A. Levine, Charles L. Bennett, J.R. Henderson, D.A. Knapp, and R.E. Marrs. Evaporative cooling of highly charged ions in EBIT. In A. Hershcovitch, editor, International Symposium on Electron Beam Ion Sources and Their Applications, AIP Conference Proceedings No. 188, pages 158-165. AIP, New York, 1989. 
[46] D.A. Knapp, R.E. Marrs, S.R. Elliott, E.W. Magee, and R. Zasadzinski. A high-energy electron beam ion trap for production of high-charge high-Z ions. Nucl. Instr. and Meth. in Phys. Res. A, 334:305-312, 1993.

|47| R.E. Marrs, L. Gruber, and E. Magee. Development of an intense EBIT. EBIT annual report 1995, Lawrence Livermore National Laboratory, Livermore, CA 94550, October 1996. UCRL-ID-124429.

[48| J.R. Pierce. Theory and Design of Electron Beams. D. van Nostrand Company Inc., Princeton, New Jersey, 2 edition, 1954.

[49| M.A. Levine, R.E. Marrs, C.L. Bennett, J.R. Henderson, D.A. Knapp, and M.B. Schneider. EBIT: Electron beam ion trap. In A. Hershcovitch, editor, International Symposium on Electron Beam Ion Sources and Their Applications, AIP Conference Proceedings No. 188, pages 82-101. AIP, New York, 1989.

[50| Manfred von Ardenne. Tabellen der Elektronenphysik, Ionenphysik und Übermikroskopie, volume 1, pages 73-75. VEB Deutscher Verlag der Wissenschaften, Berlin, 1956.

[51| O.W. Richardson and F.C. Brown. The kinetic energy of the negative electrons emitted by hot bodies. Phil. Mag., 16(XCIII):353-376, September 1908. The London, Edinburgh, and Dublin Philosophical Magazine and Journal of Science. Sixth Series.

152| Saul Dushman. Electron emission from metals as a function of temperature. Phys. Rev., 21:623-636, 1923.

[53| Christian Weißmantel and Claus Hamann. Grundlagen der Festkörperphysik, page 354. Springer Verlag, Berlin, 1980. ISBN 3-540-09072.

[54] C.D. Child. Discharge from hot CaO. Phys. Rev., 32(5):492-511, May 1911. Phys. Rev. Series No.1 (ended 1912). 
[55] Irving Langmuir. The effect of space charge and residual gases on the thermionic current in high vacuum. Phys. Rev., 2:402-403, 1913. Abstract of a paper presented at the New York meeting of lthe Physical Society, October 18, 1913.

|56| Irving Langmuir. The effect of space charge and residual gases on the thermionic current in high vacuum. Phys. Rev., 2:450-486, 1913.

[57] David Arthur Vogel. Electron Impact Ionization of Highly Charged Lithiumlike Ions. PhD thesis, Georgia Institute of Technology, August 1992. UCRL-LR111710; Available to the public from the National Technical Information Service, U.S. Department of Commerce, 5285 Port Royal Rd., Springfield, VA 22161, USA.

[58] S.B. Utter, P. Beiersdorfer, G.V. Brown, J.R. Crespo López Urrutia, K. Widmann, and A.J. Smith. Position and size of the electron beam in an electron beam ion trap. Prepared for submittal to Nucl. Instr. and Meth. in Phys. Res. B, 1998.

[59] David R. Lide and H.P.R. Frederikse. CRC Handbook of Chemistry and Physics, chapter "Superconductivity", by B.W. Roberts, page E102. CRC Press Inc., Boca Raton, FL, 70 edition, 1989-1990.

[60] A. Ashkin. Dynamics of electron beams from magnetically shielded guns. $J$. Appl. Phys., 29(11):1594-1604, November 1958.

[61| Kenneth T. Harker. Non-laminar flow in cylindrical electron beams. J. Appl. Phys., 28:645-650, June 1957.

[62] Gabriel Herrmann. Optical theory of thermal velocity effects in cylindrical electron beams. J. Appl. Phys., 29(2):127-136, February 1958.

[63] H.F. Webster. Electron physics - stucture in magnetically confined beams. $J$. Appl. Phys., 28(12):1388-1397, December 1957. 
[64| J. Larmor. Aether and Matter. Cambridge University Press, Cambridge, England, 1900. Chapter VI.

|65| Leon Brillouin. A theorem of Larmor and its importance for electrons in magnetic fields. Phys. Rev., 67(7 and 8):260-266, April 1945.

[66] Kurt Amboss. Studies of a magnetically compressed electron beam. IEEE Trans.Electron Devices, 16(11):897-904, November 1969.

[67] P.A. Lindsay. The problem of electron velocity distribution. Proc. IEEE, 51(12):1710-1722, 1963.

[68 W. Ronald Gentry and Clayton F. Giese. Ten-microsecond pulsed molecular beam source and a fast ionization detector. Rev. Sci. Instrum., 49(5):595-600, May 1978.

[69] I.G. Brown, J.E. Galvin, B.F. Gavin, and R.A. MacGill. Metal vapor vacuum arc ion source. Rev. Sci. Instrum., 57(6):1069-1084, June 1986.

|70| I.G. Brown, J.E. Galvin, R.A. MacGill, and R.T. Wright. Miniature high current metal ion source. Appl. Phys. Lett., 49(16):1019-1021, October 1986.

171 I.G. Brown, B. Feinberg, and J.E. Galvin. Multiply stripped ion generation in the metal vapor vacuum arc. J. Appl. Phys., 63(10):4889-4898, May 1988.

|72| S.R. Elliott and R.E. Marrs. A wire probe as an ion source for an electron beam ion trap. Nucl. Instr. and Meth. in Phys. Res. B, 100(4):529-535, June 1995.

[73| B.M. Penetrante, M.A. Levine, and J.N. Bardsley. Computer predictions of "evaporative" cooling of highly charged ions in EBIT. In A. Hershcovitch, editor, International Symposium on Electron Beam Ion Sources and Their Applications, AIP Conference Proceedings No. 188, pages 145-157. AIP, New York, 1989. 
$174 \mid$ J.D. Silver, A.J. Varney, H.S. Margolis, P.E.G. Baird, I.P. Grant, P.D. Groves, W.A. Hallett, A. T. Handford, P.J. Hirst, A.R. Holmes, D.J.H Howie, R.A. Hunt, K.A. Nobbs, M. Roberts, W. Studholme, J.S. Wark, M.T. Williams, M.A. Levine, D.D. Dietrich, W.G. Graham, I.D. Williams, R.O'Neil, and S.J. Rose. The Oxford electron-beam ion trap: A device for spectroscopy of highly charged ions. Rev. Sci. Instrum., 65(4):1072-1074, April 1994.

[75] J.D. Gillaspy. First results from the EBIT at NIST. Phys. Scr., T71:99-103, 1997. Proceedings of the 6th International Symposium on Electron Beam Ion Sources and their Applications, Stockholm 1994, ed. by L. Liljeby.

[76] Frederick John Curell, Junji Asada, Koichi Ishii, Arimichi Minoh, Kenji Motohashi, Nobuyuki Nakamura, Kazou Nishizawa, Shunsuke Ohtani, Kiyohiko Okazaki, Makoto Sakurai, Hiroshi Shiraishi, Seiji Tsurubuchi, and Hirofumi Watanabe. A new versatile electron-beam ion trap. J. Phys. Soc. Japan, 65(10):3186-3192, October 1996.

|77| C. Biedermann, R. Radtke, and G. Fußmann. Direct observation of x-ray emission from high-n spectator electrons following radiative stabilization in dielectronically excited highly charged barium. Phys. Rev. A, 56(4):R2522-R2525, October 1997. Rapid Communications.

|78| EG\&G ORTEC. Modular pulse-processing electronics and semiconductor radiation detectors. Product catalog, EG\&G ORTEC, Oak Ridge, TN 37831-0895, $1997 / 98$.

[79] Robley Dunglison Evans. The Atomic Nucleus, pages -973. McGraw-Hill Book Company Inc., 1955.

[80] Lynn Kissel and R.H. Pratt. Rayleigh scattering. In Bernd Crasemann, editor, Atomic Inner-Shell Physics, pages 465-532, New York, 1985. Plenum Press. 
[81] Ludwig Bergmann and Clemens Schaefer. Lehrbuch der Experimentalphysik, Band III, Optik, chapter 3 "Interferenz und Beugung", by Hans-Joachim Eichler, page 461. Walter de Gruyter, Berlin, 8 edition, 1987. Herausgeber: Heinrich Gobrecht.

[82] Max Born and Emil Wolf. Principles of Optics, chapter II "Electromagnetic Potentials and Polarization", pages 90-94. Pergamon Press, Oxford, 6 (corrected) edition, 1989.

[83] Klaus Widmann. Anwendung holographischer optischer Elemente zur Reduzierung störender Eigenstrahlung bei interferometrischen Untersuchungen von Plasmen. Diplomarbeit, Technische Universität Graz, Austria, May 1992.

[84] T. Åberg and J. Tulkki. Inelastic x-ray scattering including resonance phenomena. In Bernd Crasemann, editor, Atomic Inner-Shell Physics, pages 419-463, New York, 1985. Plenum Press.

[85] Ludwig Bergmann and Clemens Schaefer. Lehrbuch der Experimentalphysik, Band III, Optik, chapter 7 "Quantenoptik", by Horst Weber, page 840. Walter de Gruyter, Berlin, 8 edition, 1987. Herausgeber: Heinrich Gobrecht.

[86] R. Gunnick and J.B. Niday. Computerized quantitative analysis by gamma-ray spectrometry. Report UCRL-51061 Vols. I to V, Lawrence Livermore National Laboratory, University of California, 1971.

[87] Raymond Gunnick. Computer techniques for analysis of gamma-ray spectra. Report UCRL-80297, Lawrence Livermore National Laboratory, University of California, 1978.

[88] Raymond Gunnick and Wayne D. Ruther. GRPANL: a program for fitting complex peak groupings for gamma and $\mathrm{x}$-ray energies and intensities. Report UCRL-52917, Lawrence Livermore National Laboratory, University of California, January 1980. GRPANL stands for GRouP ANaLysis. 
[89] Ron Jenkins, R.W. Gould, and Dale Gedcke. Quantitative X-ray Spectrometry, chapter 6 "General Computer Applications and Quantitative Spectrum Analysis as Applied to Energy-Dispersive Analysis", page 268. Marcel Dekker, Inc., New York, 2 edition, 1995. ISBN 0-8247-9554-7.

|90| E. Friedrich, P. Knipping, and M. Laue. Interferenz-Erscheinungen bei Röntgenstrahlen. Sitzungsbericht, Bayrische Akademie der Wissenschaften, München, 1912. pg. 303-322.

[91] William Henry Bragg and William Lawrence Bragg. The reflection of $x$-rays by crystals, i. Proc. Roy. Soc. A, 88:428-438, 1913.

[92] L. von Hámos. Röntgenspektroskopie und Abbildung mittels gekrümmter Kristallreflektoren I. Geometrisch-optische Betrachtungen. Ann. Phys., $17(5): 717-725,1933$.

[93| L. von Hámos. Röntgenspektroskopie und Abbildung mittels gekrümmter Kristallreflektoren II. Beschreibung eines fokussierenden Spektrographen mit punktgetreuer Spaltabbildung. Ann. Phys., 19(5):253-260, 1934.

[94| Jesse W.M. DuMond. A high resolving power, curved-crystal focusing spectrometer for short wave-length $\mathrm{x}$-rays and gamma-rays. Rev. Sci. Instrum., 18(9):626-638, September 1947.

[95] P.P. Ewald. Introduction to the dynamical theory of x-ray diffraction. Acta Crystallogr. A, 25:103-108, 1969.

[96] A.H. Compton and S.K. Allison. $X$-Rays in Theory and Experiment. page 674. D. van Nostrand Company, Inc., Princeton, New Jersey, 2 edition, 1935. reprinted 1963. 
[97| E. Källne, J. Källne, L.G. Atencio, J. Chmielewski, G. Idzorek, and C.L. Morris. High-count-rate proportional counter for soft $\mathrm{x}$-ray position detection. Rev. Sci. Instrum., 58(6):1077-1086, June 1987.

[98| D. Vogel, P. Beiersdorfer, V. Decaux, and K. Widmann. High-pressure positionsensitive proportional counter. Rev. Sci. Instrum., 66(1):776-778, 1995.

[99| G. Borrmann. Die Absorption von Röntgenstrahlen im Fall der Interferenz. Z. Phys., 127:297-323, 1950.

[100| K.T. Cheng; M.H. Chen, W.R. Johnson, and J. Sapirstein. Relativistic configuration-interaction calculations for the ground state and $n=2$ singlet states of heliumlike ions. Phys. Rev. A, 50(1):247-255, July 1994.

[101| Janos Kirz, David T. Attwood, Burton L. Henke, Malcolm R. Howells, Kurt D. Kennedy, Kwang-Je Kim, Jeffrey B. Kortright, Rupert C. Perera, Piero Pianetta, John C. Riordan, James H. Scofield, Gary L. Stradling, Albert C. Thompson, James H. Underwood, Gwyn P. Williams, and Herman Winick. $\mathrm{X}$-ray data booklet. Center for X-ray Optics, Lawrence Berkeley National Laboratory, University of California, Berkeley, California 94720, April 1986.

[102] Günter Zschornack. Atomdaten, für die Röntgenspektralanalyse. SpringerVerlag, Berlin, 1989.

[103| M. Bitter, H. Hsuan, C. Bush, S. Cohen, C.J. Cummings, B. Grek, K.W: Hill, J. Schivell, M. Zarnstorff, P. Beiersdorfer, A. Osterheld, A. Smith, , and B. Fraenkel. Spectra of heliumlike krypton from tokamak fusion test reactor plasmas. Phys. Rev. Lett., 71(7):1007-1010, August 1993.

|104| B. Yaakobi, F.J. Marshall, and R. Epstein. High temperature of lasercompressed shells measured with $\mathrm{Kr}^{34+}$ and $\mathrm{Kr}^{35+}$ x-ray lines. Phys. Rev. E, 54(5):5848-5850, November 1996. 
[105] J. Cummings, S.A. Cohen, R. Hulse, D.E. Post, M.H. Redi, and J. Perkins. Power radiated from ITER by impurities. Journal of Nuclear Materials, 176:916-921, December 1990.

[106| L.D. Horton. Atomic and molecular data needs for fusion research. Phys. Scr., T65:175-178, 1996.

[107| P.Indelicato, J.P. Briand, M. Tavernier, and D. Liesen. Experimental study of relaticistic correlaions and QED effects in heliumlike krypton ions. Z. Phys. D, 2(6):249-250, 1986. Short Note.

[108] A. Burek. Crystals for astronomical x-ray spectroscopy. Space Science Instrum., 2:53-104, 1976.

[109| N.G. Alexandropoulos and G.G. Cohen. Crystals for stellar spectrometers. Appl. Spectr., 28(2):155-164, 1974.

|110| P. Beiersdorfer, V. Decaux, S. Elliott, K. Widmann, and K. Wong. Temperature of the ions produced and trapped in an electron-beam ion trap. Rev. Sci. Instrum., 66(1):303-305, January 1995.

[111| P. Beiersdorfer, V. Decaux, and K. Widmann. Measurement of the temperature of cold highly charged ions produced in an electron beam ion trap. Nucl. Instr. and Meth. in Phys. Res. B, 98:566-568, 1995.

[112] P.J. Mohr. Energy levels of hydrogen-like atoms predicted by quantum electrodynamics, $10 \leq Z \leq 40$. At. Data Nucl. Data Tables, 29(3):453-466, November 1983.

[113] P. Beiersdorfer, T. Phillips, V.L. Jacobs, K.W. Hill, M. Bitter, S. von Goeler, and S.M. Kahn. High-resolution measurements, line identification, and spectral modeling of K- $\alpha$.transitions in Fe-XVIII-Fe-XXV. Astrophys. J., 409(2):846859, June 1993. 
[114] Arthur H. Compton and Samuel K. Allison. X-Rays in Theory and Experiment. page 280. D. van Nostrand Company, Inc., Princeton, New Jersey, 2 edition, 1935. reprinted 1963.

[115] M.H. Chen, K.T. Cheng, and W.R. Johnson. Relativistic configurationinteraction calculations of $\mathrm{n}=2$ triplet states of heliumlike ions. Phys. Rev. A, 47(5):3692-3703, May 1993.

[116] M. Bitter, H. Hsuan, K.W. Hill, R. Hulse, M. Zarnstorff, and P. Beiersdorfer. Xray spectra of heliumlike krypton as a potential ion temperature diagnostic for the international thermonuclear experimental reactor (ITER). In R.K. Janev, editor, Atomic and Plasma-Material Interactions Processes in Controlled Thermonuclear Fusion. Elsevier, 1993.

|117| L.A. Vainshtein and U.I. Safronova. Technical Report Report No.2, Lebedev Institute of Spectroscopy, 1985.

[118| T. Mooney, E. Lindroth, P. Indelicato, E.G. Kessler Jr., and R.D. Deslattes. Precision measurements of K-transitions and L-transitions in xenon - experiment and theory for the K-levels, L-levels, and M-levels. Phys. Rev. A, 45(3):1531-1543, February 1992.

[119] James H. Scofield. Angular and polarization correlations in photoionization and radiative recombination. Phys. Rev. A, 40(6):3054-3060, September 1989.

[120] E.B. Saloman, J.H. Hubell, and J.H. Scofield. X-ray attenuation cross sections for energies $100 \mathrm{eV}$ to $100 \mathrm{keV}$ and elements $\mathrm{Z}=1$ to $\mathrm{Z}=92$. At. Data Nucl. Data Tables, 38(1):1-197, January 1988.

[121] T. Stöhlker, P.H. Mokler, C. Kozhuharov, and A. Warczak. Photoelectric effect in the relativistic domain revealed by the time-reversed process for highly charged uranium ions. Comments on Atomic and Molecular Phys., 33(5):271, 1997. 
[122] R.M. Steffen and K. Alder. The electromagnetic interaction in nuclear spectroscopy. In W.D. Hamilton, editor, The electromagnetic interaction in nuclear spectroscopy, page 505, New York, 1975. North Holland.

[123] P. Beiersdorfer, D.A. Vogel, K.J. Reed, V. Decaux, J.H. Scofield, K. Widmann, G. Hölzer, E. Förster, O. Wehrhan, D.W. Savin, and L. Schweikhard. Measurement and interpretation of the polarization of the $\mathrm{x}$-ray line emission of heliumlike Fe XXV excited by and electron beam. Phys. Rev. A, 53(6):39743981, June 1996.

[124] HL. Zhang, D.H. Sampson, and R.E.H. Clark. Relativistic cross sections for excitation of highly charged ions to specific magnetic sublevels by an electron beam. Phys. Rev. A, 41(1):198-206, January 1990.

[125] I.C. Percival and M.J. Seaton. The polarization of atomic line radiation excited by electron impact. Phil. Trans. Royal Soc. London, 251(990):113-138, November 1958.

[126] Wolfgang Lotz. Electron-impact ionization cross-sections and ionization rate coefficients for atoms and ions. Astrophys. J., XVI(128):207-238, May 1967. Supplement Series.

[127] Wolfgang Lotz. Electron-impact ionization cross-sections and ionization rate coefficients for atoms and ions from hydrogen to calcium. Z. Phys., 216:241247. 1968.

|128| P. Beiersdorfer, G.V. Brown, J.R. Crespo López-Urrutia, R. Olson, L. Schweikhard, and K. Widmann. Charge transfer between neutral gases and cold highly charged ions. In Atomic Processes in Plasmas. APS, AIP, March 1998. 11th Topical Conference on Atomic Processes in Plasmas, Auburn, Alabama, 22-26 March, 1998. 
[129] R.E. Marrs, S.R. Elliott, and J.H. Scofield. Measurement of electron-impact ionization cross sections for hydrogenlike high-Z ions. Phys. Rev. A, 56(2):13381345, August 1997.

|130| W.R. Johnson. Radiative decay rates of metastable one-electron atoms. Phys. Rev. Lett., 29(17):1123-1126, October 1972.

[131] S.P. Goldman and G.W.F. Drake. Relativistic two-photon decay rates of $2 s_{1 / 2}$ hydrogenlike ions. Phys. Rev. A, 24(1):183-191, July 1981.

[132| G.W.F. Drake. Spontaneous two-photon decay rates in hydrogenlike and heliumlike ions. Phys. Rev. A, 34(4):2871-2880, October 1986.

[133| C.D. Lin, W.R. Johnson, and A. Dalgarno. Radiative decays of the $\mathrm{n}=2$ states of He-like ions. Phys. Rev. A, 15:154-161, 1977.

[134| P. Beiersdorfer, L. Schweikhard, J.R. Crespo López-Urrutia, and K. Widmann. The magnetic trapping mode of an Electron Beam Ion Trap: New opportunities for highly charged ion research. Rev. Sci. Instrum., 67(11):3818-3826, November 1996.

[135] S. Chantrenne, P. Beiersdorfer, R. Cauble, and M.B. Schneider. Measurement of electron impact excitation cross sections for heliumlike titanium. Phys. Rev. Lett., 69(2):265-268, July 1992.

[136| K.L. Wong, P. Beiersdorfer, K.J. Reed, and D.A. Vogel. Electron-impact excitation cross-section measurements of highly charged heliumlike and lithiumlike ions. Phys. Rev. A, 51(2):1214-1220, February 1995.

[137| T. Stöhlker, P.H. Mokler, K. Beckert, F. Bosch, H. Eickhoff, B. Franzke, M. Jung, T. Kandler, O. Klepper, C. Kozhuharov, R. Moshammer, F. Nolden, H. Reich, P. Rymuza, P. Stadtke, and M. Steck. Ground-state Lamb shift for 
hydrogenlike uranium measured at the ESR storage ring. Phys. Rev. Lett., 71(14):2184-2187, October 1993.

[138] J.P. Briand. Present status and prospect of the experimental study of QED in high-Z ions. Phys. Scr., T46:157-161, 1993.

[139| J. Schweppe, A. Belkacem, L. Blumenfeld, N. Claytor, B. Feinberg, H. Gould, V.E. Kostroun, L. Levy, S. Misawa, J.R. Mowat, and M.H. Prior. Measurement of the Lamb shift in lithiumlike uranium $\left(\mathrm{U}^{89+}\right)$. Phys. Rev. Lett., 66(11):14341437, March 1991.

[140| P. Beiersdorfer, D. Knapp, R.E. Marrs, S.R. Elliott, and M.H. Chen. Structure and Lamb shift of $2 p_{3 / 2}-2 s_{1 / 2}$ levels in lithiumlike $\mathrm{U}^{89+}$ through neonlike $\mathrm{U}^{82+}$. Phys. Rev. Lett., 71(24):3939-3942, December 1993.

[141] P. Beiersdorfer, A. Osterheld, S.R. Elliott, M.H. Chen, D. Knapp, and K. Reed. Structure and Lamb shift of $2 p_{3 / 2}-2 s_{1 / 2}$ levels in lithiumlike $\mathrm{Th}^{87+}$ through neonlike $\mathrm{Th}^{80+}$. Phys. Rev. A, 52:2693-2706, 1995.

[142] P. Beiersdorfer, A.L. Osterheld, J.H. Scofield, J.R. Crespo López-Urrutia, and K. Widmann. Measurement of qed and hyperfine splitting in the $2 s_{1 / 2} \leftarrow 2 p_{3 / 2}$ x-ray transition in ${ }^{209} \mathrm{Bi}^{80+}$. Phys. Rev. Lett., 80(6):3022-3025, April 1998.

[143] David R. Lide and H.P.R. Frederikse. CRC Handbook of Chemistry and Physics, chapter "X-ray Wavelengths", pages E152-E190. CRC Press Inc., Boca Raton, FL, 70 edition, 1989-1990.

[144] J.A. Bearden. X-ray wavelengths. Rev. Mod. Phys., 39(1):78-124, January 1967.

[145] E. Richard Cohen and Barry N. Taylor. The 1986 adjustment of the fundamental physical constants. Rev. Mod. Phys., 59(4):1121-1148, October 1987. 
[146] E.G. Kessler Jr., A. Henins, R.D. Deslattes, L. Nielsen, and M. Arif. Precision comparison of the lattice parameters of silicon monocrystals. J. Res. Nat. Inst. Stand. Technol., 99(1):1-18;285, February 1994. pp. 1-18 article; p. 285 Errata to this article.

[147| P. Becker, U. Kuetgens, J. Stumpel, S. Biernacki, and M. Scheffler. The silicon28 lattice parameter. PTB-Mitteilungen, 105(2):95-98, April 1995.

[148| E.G. Kessler Jr., R.D. Deslattes, A. Henins, and W.C. Sauder. Redetermination of ${ }^{198} \mathrm{Au}$ and ${ }^{192} \mathrm{Ir} \gamma$-ray standard between 0.1 and $1.0 \mathrm{MeV}$. Phys. Rev. Lett., 40(3):171-174, January 1978.

[149| E.G. Kessler Jr., R.D. Deslattes, and A. Henins. Wavelength of the W K $\alpha_{1}$ x-ray line. Phys. Rev. A, 19(1):215-218, January 1979.

$\lceil 150 \mid$ E.G. Kessler Jr., R.D. Deslattes, D. Girard, W. Schwitz, L. Jacobs, and O. Renner. Mid-to-high-Z precision $\mathrm{x}$-ray measurements. Phys. Rev. A, 26(5):26962706, November 1982.

[151] P. Beiersdorfer. Spectral measurements of few-electron uranium ions produced and trapped in a high-energy electron beam ion trap. Nucl. Instr. and Meth. in Phys. Res. B, 99(1-4):114-116, May 1995.

[152| K. Widmann, P. Beiersdorfer, V. Decaux, and M. Bitter. Measurements of the $\mathrm{K} \alpha$ transition energies of heliumlike krypton. Phys. Rev. A, 53(4):2200-2205, April 1996.

[153| H. Tillborg, A. Nilsson, and N. Martensson. Shake-up and shake-off structures in core level photoemission spactra from adsorbates. J. Electron Spectrosc. Relat. Phenom., 62:73-93, March 1993. 
[154| G. Hölzer, M. Fritsch, M.Deutsch, J. Härtwig, and E. Förster. K $\alpha_{1,2}$ and $\mathrm{K} \beta_{1,3}$ $\mathrm{x}$-ray emission lines of the $3 d$ transition metals. Phys. Rev. A, 56(6):4554-4568, December 1997.

[155] J.P. Briand, P. Indelicato, A. Simionovici, V. San Vicente, D. Liesen, and D. Dietrich. Spectroscopic study of hydrogenlike and heliumlike xenon ions. Europhys. Lett., 9(3):225-230, June 1989.

[156] R.E. Marrs, S.R. Elliott, and Th. Stöhlker. Measurement of two-electron contributions to the ground-state energy of heliumlike ions. Phys. Rev. A, 52(5):35773585, November 1995. 


\section{Acknowledgments}

Foremost, I wish to thank my supervisor and EBIT spectroscopy group leader, Peter Beiersdorfer, who was available 24 hours a day, seven days a week for support regarding questions in the experimental and theoretical field of physics. My knowledge of atomic physics has been helped tremendously through discussions with him. Moreover, I am thankful for his administrative support, which allowed me to focus on my research.

I also want to express my gratitude to my advisor and Doktorvater, Prof. Helmut Jäger, who respected and supported my decision of gaining working experience outside of Austria and always offered immediate help and encouraged a fast forthcoming of my thesis, e.g., by accepting a thesis written in English - a benefit, that most of my German colleagues here in Livermore could not enjoy. I wish to thanks Prof. Bernhard Schnizer for his prompt evaluation of my thesis. I also owe a lot to UDoz. Theo Neger, who has been most supportive in so many ways. Having had a chance to work with him was an inspiration and motivation which kept on throughout the years. I also want to acknowledge a tremendous debt to my colleagues at the Institute for Experimental Physics at the University of Technology in Graz, in particular, to Jürgen Flieser, who helped to overcome all bureaucratic hurdles, built by the Austrian University system, that usually would make it impossible for a student to gain longtime international experience. Jürgen also volunteered to solve the US-Letter-sizeDIN-A4 clinch. Thanks.

New country, new habits, same language, and an outstanding work ethic. That's the short version of my EBIT experience. Thanks to the EBIT project leader, Dieter Schneider, the years I have spent at EBIT have been years immersed in an excellent working environment. Dieter always keeps an eye on his crew, especially on the crew's future. It is my understanding that Dieter's effort in keeping the special EBIT team spirit is strongly supported by the Director of the Physics and Space Technology department, Dick Fortner. A special thanks, thus, belongs to him. One corner stone of EBIT's success is the commitment and excellent skills of the technicians, who are 
in charge of maintaining, improving, adjusting, repairing, and changing the EBIT facility and components of the EBIT facility, respectively. In particular, I wish to thank Dan Nelson for his prompt service and his patience regarding the ever changing experimental circumstances, and Ed Magee for his help in the mechanical design and the manufacturing of all the spectrometer parts, and for his assistance in translating between the metric and US "language" of metrology. Thanks also to Ken Visbeck for his watchiful eye on the cryogenics, needed for all solid state $\mathrm{x}$-ray detectors, and to Phil D'Antonio for keeping the electronics working and constantly updated.

My gratitude shall always accompany my colleagues, co-workers, fellow students, office mates. Among them: Greg Brown, with whom I shared the office and many fruitful discussions and who helped out on numerous occasions with practical advice, constructive criticism, and an uncountable number of shifts. Jose Crespo LópezUrrutia, whose tremendous energy and pervicaciousness inspired the whole EBIT project in many ways. Although his approach to overcome the various experimental obstacles is sometimes strenuous, I am truly thankful for the many helpful hints I have received throughout the years I have been working with Joserra. Steve Utter, the "newest" addition to the spectroscopy group, whose team spirit and humor are certainly motivating. I am also glad that I had a chance to work with Steve Elliott, Vince Decaux, Bob Thoe, and, especially, Manfred Bitter.

The EBIT project nourishes several different physics communities and the relatively small size of the facility gave me the opportunity of numerous interesting discussions on physics for which I am very grateful, especially to Ross Marrs, Alan Barnes, Alex Hamza, Thomas Schenkel, Mike Briere, Dave Knapp, Joe McDonald, Joachim Steiger, Bret Beck, and, last but not least, Lukas Gruber. I am also thankful to Christiane Rühlicke for providing a friendly, motivating, and efficient working environment in the office, and the opportunity to improve my German. Thanks also to Sophie Bardin and Bruce Birkett for their contribution to my pleasant office experience. I am grateful to Mau Chen, Al Osterheld, Kennedy Reed, and Jim Scofield who 
have calculated most of the atomic data presented in this thesis: During the first two years of my almost five-year Graduate Student Term Employment, the EBIT program was part of V-Division, which, at that time, was lead by Andy Hazi. I would like to thank him for his support. A place like the Lawrence Livermore National Laboratory offers diversity, creativity, and exposes the workforce to many things that are not necessarily limited to the world of physics. Due to the size and the original purpose of this laboratory a lack of flexibility can be experienced as well as an unbalanced distribution between the productive and administrative part with respect to the funding. However, only places like the LLNL have enough strength to push the scientific frontiers in so many different disciplines and enable interdisciplinary interaction for the motivated and interested part of the work force. Thus, I want to acknowledge that this work was performed under the auspices of the United States Department of Energy by Lawrence Livermore National Laboratory under contract number W-7405-ENG-48 and supported during the first year by the Office of Fusion Energy of the Department of Energy, Division of Applied Plasma Physics.

Also a special thanks to Trish Dobson from the Space Science Laboratory at UC Berkeley for her support regarding my position as a Junior Research Specialist in her group.

The years in California have been a great experience, although I, perhaps, have not seen as much of the State as a tourist, who stays for only two to three weeks. However, those years not only opened the window on the physics of highly charged ions but also on a different chapter in my life. Ending my bachelor years by marrying my most beloved one was certainly the emotional highlight of my Graduate Student years and I wish to express a tremendous debt to my wife for having neglected most of my promises, especially during the last year in which my thesis received absolute priority. Finally, I wish to thank my whole family: in particular. my parents, who always respected my decisions and gave me their full support, no matter how many miles we are apart. 